\title{
Divergent Synthesis of Antiviral Diterpenes Wickerols A and B
}

\author{
Jiachen Deng, Yuhan Ning, Hailong Tian and Jinghan Gui*
}

CAS Key Laboratory of Synthetic Chemistry of Natural Substances, Center for Excellence in Molecular Synthesis, Shanghai Institute of Organic Chemistry, University of Chinese Academy of Sciences, Chinese Academy of Sciences, 345 Lingling Road, Shanghai 200032, China

*Correspondence to: guijh@ sioc.ac.cn

\section{Supporting Information}

\section{Table of Contents}

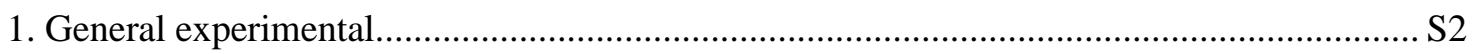

2. Optimization of $\mathrm{SmI}_{2}$-mediated ketone-allylic acetate reductive cyclization. ...................... S3

3. Stereochemical rationale for the $\mathrm{SmI}_{2}$-mediated cyclization............................................ 4

4. Stereochemical rationale for the Claisen rearrangement ................................................. 4

5. Experimental procedures and characterization data for compounds 1-23......................... S5

6. NMR comparison of synthetic and natural wickerols A and B ...................................... S24

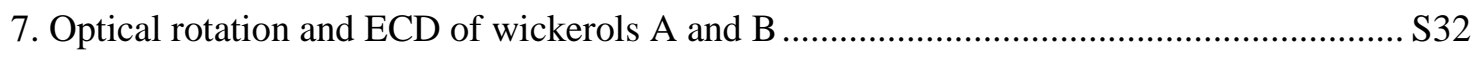

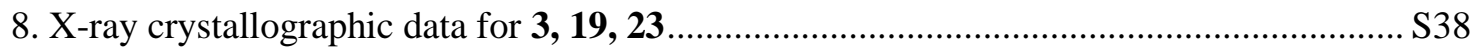

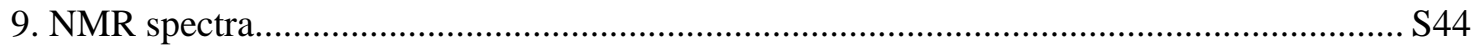

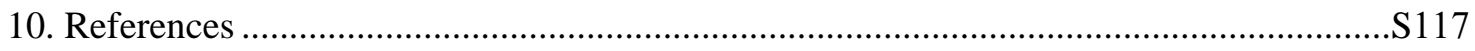




\section{General experimental}

All reactions utilizing air- or moisture-sensitive reagents were carried out in flame-dried glassware under an argon atmosphere, unless otherwise stated. Dry tetrahydrofuran (THF), dichloromethane (DCM), toluene (PhMe), diethyl ether $\left(\mathrm{Et}_{2} \mathrm{O}\right)$ were obtained by passing the HPLC grade or pre-dried solvents through activated alumina columns. Reagents were purchased at the highest commercial quality and used without further purification, unless otherwise stated. Reactions were magnetically stirred and monitored by thin layer chromatography (TLC) with $0.15-0.2 \mathrm{~mm}$ pre-coated silica gel $(10-40 \mu \mathrm{m})$ plates, using UV light as the visualizing agent or ethanolic phosphomolybdic acid or $p$-anisaldehyde and heating as developing agents. Flash chromatography was performed with silica gel (200300 mesh) under pressure. Yields refer to chromatographically and spectroscopically $\left({ }^{1} \mathrm{H}\right.$ NMR) homogeneous material, unless otherwise stated. NMR spectra were recorded on Bruker-400 or Bruker-500 spectrometers. ${ }^{1} \mathrm{H}$ NMR spectra were calibrated using residual undeuterated solvent as an internal reference $\left(\mathrm{CDCl}_{3}: \delta=7.26 \mathrm{ppm} ; \mathrm{MeOH}-d_{4}: \delta=4.87\right.$ ppm) and ${ }^{13} \mathrm{C}$ NMR spectra were calibrated against the deuterated solvent peak $\left(\mathrm{CDCl}_{3}: \delta=\right.$ $77.2 \mathrm{ppm} ; \mathrm{MeOH}-d_{4}: \delta=49.0 \mathrm{ppm}$ ). The following abbreviations were used to explain multiplicities: $\mathrm{s}=$ singlet, $\mathrm{d}=$ doublet, $\mathrm{t}=$ triplet, $\mathrm{q}=$ quartet, $\mathrm{m}=$ multiplet, $\mathrm{br}=$ broad. $\mathrm{IR}$ spectra were collected on Avatar 330 FT-IR spectrometer. Melting points were determined on JIAHANG JH40 microscopic melting point apparatus and were uncorrected. Optical rotations were determined on Anton Paar MCP 5500 Polarimeter in the solvent indicated. High-resolution mass spectra were recorded on IonSpec 4.7 Tesla FTMS or Bruker Daltonics, Inc. APEXIII 7.0 TESLA FTMS. 
2. Optimization of $\mathrm{SmI}_{2}$-mediated ketone-allylic acetate reductive cyclization.

Table S1. Optimization of SmI2-mediated ketone-allylic acetate reductive cyclization

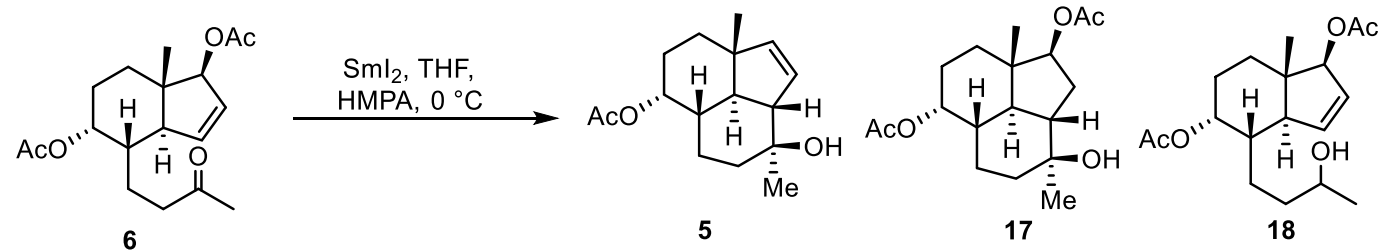

\begin{tabular}{|c|c|c|c|c|c|c|c|}
\hline \multirow{2}{*}{ entry } & \multirow{2}{*}{$\begin{array}{l}\text { scale } \\
(\mathrm{mg})\end{array}$} & \multirow{2}{*}{$\begin{array}{c}\mathrm{SmI}_{2} \\
\text { (equiv) }\end{array}$} & \multirow{2}{*}{ additive (equiv) } & \multirow{2}{*}{ temp. $\left({ }^{\circ} \mathrm{C}\right)$} & \multicolumn{3}{|c|}{${ }^{1} \mathrm{H}$ NMR ratio ${ }^{a}$} \\
\hline & & & & & 5 & 17 & 18 \\
\hline 1 & 50 & 3.3 & HMPA (15) & 0 & 40 & 28 & 32 \\
\hline 2 & 50 & 3.3 & DMPU (15) & 0 & \multicolumn{3}{|c|}{ low conversion } \\
\hline 3 & 50 & 3.3 & ${ }^{t} \mathrm{BuOH}(15)$ & 0 & \multicolumn{3}{|c|}{ no reaction } \\
\hline 4 & 50 & 3.3 & HMPA (15), LiCl (5) & 0 & 0 & 0 & $(82)$ \\
\hline 5 & 50 & 3.3 & HMPA (15) & $-20{ }^{\circ} \mathrm{C}$ & 13 & 11 & 76 \\
\hline 6 & 50 & 3.3 & HMPA (15) & $\mathrm{rt}$ & 16 & 16 & 68 \\
\hline 7 & 50 & 3.3 & HMPA (15) & $40^{\circ} \mathrm{C}$ & 13 & 16 & 71 \\
\hline 8 & 50 & 3.3 & HMPA (10) & 0 & 30 & 21 & 49 \\
\hline 9 & 50 & 3.3 & HMPA (20) & 0 & 25 & 24 & 51 \\
\hline 10 & 50 & 3.3 & HMPA (25) & 0 & 28 & 43 & 29 \\
\hline 11 & 50 & 3.3 & HMPA (50) & 0 & 25 & 56 & 19 \\
\hline 12 & 50 & 2.5 & HMPA (15) & 0 & 21 & 20 & 59 \\
\hline 13 & 50 & 4.0 & HMPA (15) & 0 & 26 & 14 & 60 \\
\hline 14 & 50 & 4.5 & HMPA (15) & 0 & 11 & 6 & 83 \\
\hline 15 & 50 & 2.2 & HMPA (10) & 0 & trace & trace & $(75)^{b}$ \\
\hline 16 & 50 & 4.4 & HMPA (20) & 0 & 17 & 8 & 75 \\
\hline 17 & 50 & 5.5 & HMPA (25) & 0 & 26 & 11 & 63 \\
\hline 18 & 50 & 6.6 & HMPA (30) & 0 & 13 & 7 & 80 \\
\hline 19 & 322 & 3.3 & HMPA (15) & 0 & (39) & (36) & (13) \\
\hline 20 & 200 & 4.4 & HMPA (20) & 0 & $(40)$ & (27) & (28) \\
\hline 21 & 200 & 5.5 & HMPA (25) & 0 & $(52)$ & (28) & (18) \\
\hline 22 & 200 & 6.6 & HMPA (30) & 0 & (48) & (28) & $(21)$ \\
\hline 23 & 1000 & 3.3 & HMPA (15) & 0 & (41) & (35) & (12) \\
\hline 24 & 500 & 5.5 & HMPA (25) & 0 & $(52)$ & (34) & (12) \\
\hline 25 & 1000 & 5.5 & HMPA (25) & 0 & (52) & (31) & (11) \\
\hline
\end{tabular}

${ }^{a}$ Isolated yields were shown in parentheses. ${ }^{b} 25 \% \mathrm{SM}$ recovered. 


\section{Stereochemical rationale for the $\mathrm{SmI} 2$-mediated cyclization}

As shown in Scheme $\mathrm{S} 1$, the stereochemical outcome of the $\mathrm{SmI}_{2}$-mediated cyclization could be rationalized from a simplified chair-like transition structure. In the disfavored transition structure, the OSm(III)(HMPA)n group assumes a pseudo-axial orientation, thereby suffering a destabilizing 1,3-diaxial interaction.
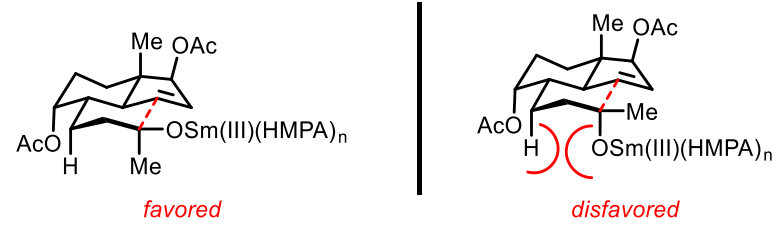

\section{Scheme S1. Stereochemical rationale for the $\mathbf{S m I}_{2}$-mediated cyclization}

\section{Stereochemical rationale for the Claisen rearrangement}

As shown in Scheme S2, the facial selectivity of the Claisen rearrangement (related to the stereochemistry at C5a) was controlled by the C2a-stereogenic center. For the undesired product A, C2a$\mathrm{H}$ and C5a-butenyl group were in trans relationship, which made $\mathrm{C}$ ring highly twisted.
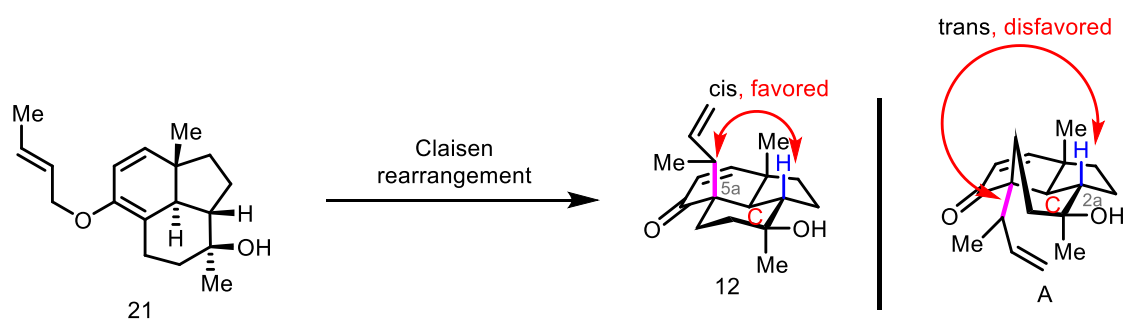

Scheme S2. Stereochemical rationale for the diastereoselectivity at C5a

As shown in Scheme S3, the high diastereoselectivity at C6 could be explained by chair-like transition state being favored over boat-like transition state, which gave rise to $\mathbf{1 2}$ instead of epi-12.

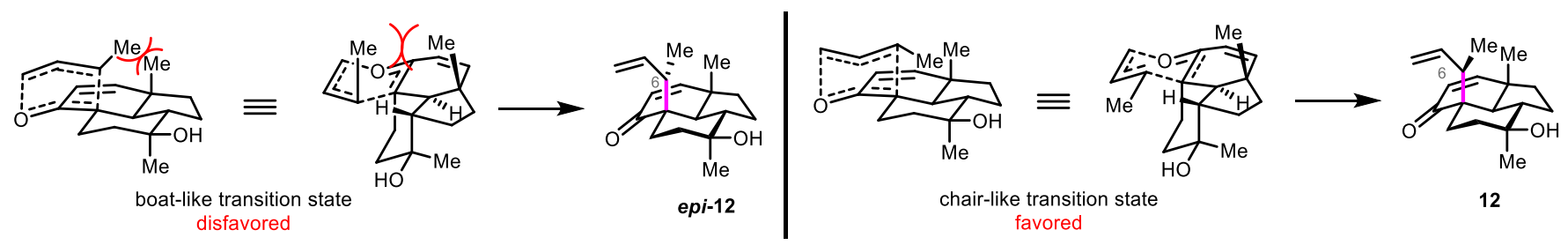

Scheme S3. Stereochemical rationale for the diastereoselectivity at C6 
5. Experimental procedures and characterization data for compounds 1-23.
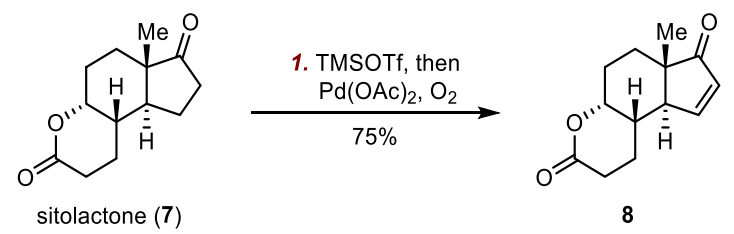

To two $500 \mathrm{~mL}$ dry round-bottom flasks with stir bars was added sitolactone $(7.0 \mathrm{~g} \times 2,31.5 \mathrm{mmol} \times 2,1.0$ equiv). Dry DCM (250 mL), Et ${ }_{3} \mathrm{~N}$ (17.5 mL, 126.0 mmol, 4.0 equiv) and TMSOTf (11.4 mL, $63.0 \mathrm{mmol}$, 2.0 equiv) were added sequentially to each flask at $0^{\circ} \mathrm{C}$ under argon. After $2 \mathrm{~h}$, additional TMSOTf (1.1 $\mathrm{mL}, 6.3 \mathrm{mmol}, 0.2$ equiv) was added. The reaction was completed after another $1 \mathrm{~h}$, as monitored by TLC (developed with $p$-anisaldehyde and heat). The combined reaction mixture was quenched with saturated aq. $\mathrm{NaHCO}_{3}(250 \mathrm{~mL})$, extracted with $\mathrm{DCM}(3 \times 300 \mathrm{~mL})$, washed with brine, dried over $\mathrm{Na}_{2} \mathrm{SO}_{4}$, and concentrated in vacuo to afford the crude silyl enol ether.

To a solution of the crude intermediate in $500 \mathrm{~mL}$ DMSO was added $\mathrm{Pd}(\mathrm{OAc})_{2}(5.7 \mathrm{~g}, 25.2 \mathrm{mmol}, 0.4$ equiv). The reaction mixture was heated at $60^{\circ} \mathrm{C}$ while oxygen was being passed through the solution. After $3.5 \mathrm{~h}$, reaction mixture was quenched with brine $(1000 \mathrm{~mL})$, extracted with EtOAc $(3 \times 300 \mathrm{~mL})$. The combined organic layers were washed with brine $(500 \mathrm{~mL})$, and the aqueous phase was extracted with additional EtOAc $(3 \times 300 \mathrm{~mL})$. The combined organic layers were dried over $\mathrm{Na}_{2} \mathrm{SO}_{4}$, and concentrated in vacuo. The crude residue was purified by flash chromatography $\left(\mathrm{SiO}_{2}, \mathrm{PE}:\right.$ EtOAc $=$ $3: 1 \rightarrow 2: 1 \rightarrow 1.5: 1 \rightarrow 1: 1)$ to afford compound $8(10.4 \mathrm{~g}, 75 \%)$.

Compound 8: white solid; $R_{f}=0.31$ (silica gel, 1:1 PE:EtOAc); $[\alpha]_{\mathrm{D}}^{23}+13.5\left(c 1.38, \mathrm{CHCl}_{3}\right) ; \mathrm{mp} 193.5$ ${ }^{\circ} \mathrm{C}$; IR(KBr) 2964, 2928, 2878, 1727, 1706, 1469, 1361, 1240, $1194 \mathrm{~cm}^{-1} ;{ }^{1} \mathrm{H} \mathrm{NMR}\left(400 \mathrm{MHz}, \mathrm{CDCl}_{3}\right) \delta$ $7.46(\mathrm{ddd}, J=6.1,1.7,0.7 \mathrm{~Hz}, 1 \mathrm{H}), 6.14(\mathrm{dd}, J=6.0,3.2 \mathrm{~Hz}, 1 \mathrm{H}), 4.59$ (q, $J=3.1 \mathrm{~Hz}, 1 \mathrm{H}), 2.82(\mathrm{ddd}, J$ $=12.7,3.0,1.9 \mathrm{~Hz}, 1 \mathrm{H}), 2.66-2.44(\mathrm{~m}, 2 \mathrm{H}), 2.36(\mathrm{ddt}, J=12.5,7.7,3.2 \mathrm{~Hz}, 1 \mathrm{H}), 2.28(\mathrm{dtd}, J=14.1$, 7.7, $6.1 \mathrm{~Hz}, 1 \mathrm{H}), 2.16$ (ddt, $J=15.5,4.5,2.1 \mathrm{~Hz}, 1 \mathrm{H}), 2.01$ (dddd, $J=15.4,12.5,5.7,3.9 \mathrm{~Hz}, 1 \mathrm{H}), 1.93-$ $1.84(\mathrm{~m}, 1 \mathrm{H}), 1.81(\mathrm{dd}, J=12.8,4.9 \mathrm{~Hz}, 1 \mathrm{H}), 1.74(\mathrm{ddd}, J=13.2,5.7,2.0 \mathrm{~Hz}, 1 \mathrm{H}), 1.12(\mathrm{~s}, 3 \mathrm{H}) \mathrm{ppm} ;{ }^{13} \mathrm{C}$ NMR $\left(101 \mathrm{MHz}, \mathrm{CDCl}_{3}\right) \delta 211.4,172.3,156.8,133.0,77.6,51.2,48.2,30.2,26.9,26.7,24.8,21.2,19.7$ ppm; HRMS (ESI-TOF, $m / z$ ): calcd for $\mathrm{C}_{13} \mathrm{H}_{17} \mathrm{O}_{3}[\mathrm{M}+\mathrm{H}]^{+}$221.1183, found 221.1174.
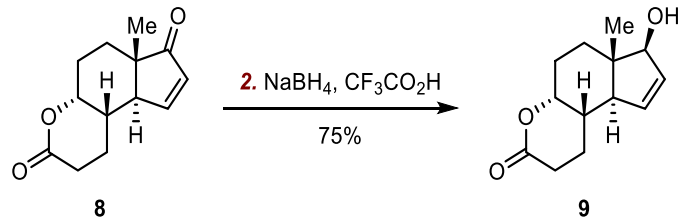

To a solution of TFA (40.6 mL, $546.0 \mathrm{mmol}, 30.0$ equiv) in $240 \mathrm{~mL} \mathrm{DCM}$ was added NaBH $4(2.8 \mathrm{~g}, 72.6$ 
mmol, 4.0 equiv) at $-10^{\circ} \mathrm{C}$, and the resulting solution was stirred for $10 \mathrm{~min}$ to dissolve $\mathrm{NaBH}_{4}$. A solution of compound 8 (4.0 g, $18.2 \mathrm{mmol}, 1.0$ equiv) in $80 \mathrm{~mL}$ DCM was added dropwise. After $15 \mathrm{~min}$, the reaction mixture was warmed to $0{ }^{\circ} \mathrm{C}$ and stirred for another $15 \mathrm{~min}$. The reaction was slowly quenched with saturated aq. $\mathrm{Na}_{2} \mathrm{CO}_{3}$ and the aqueous phase was extracted with DCM $(3 \times 300 \mathrm{~mL})$. The combined organic layers were washed with brine $(150 \mathrm{~mL})$, dried over $\mathrm{Na}_{2} \mathrm{SO}_{4}$, and concentrated in vacuo. The crude residue was purified by flash chromatography $\left(\mathrm{SiO}_{2}, \mathrm{PE}: \mathrm{EtOAc}=2: 1 \rightarrow 1: 1\right)$ to afford compound 9 $(3.0 \mathrm{~g}, 75 \%)$.

Compound 9: white solid; $R_{f}=0.35$ (silica gel, 1:1 PE:EtOAc); $[\alpha]_{\mathrm{D}}^{26}+12.8\left(c\right.$ 1.04, $\left.\mathrm{CHCl}_{3}\right) ;$ mp 164.3 ${ }^{\circ} \mathrm{C}$; IR (KBr) 3456, 2929, 2859, 1729, 1465, 1363, 1253, $1092 \mathrm{~cm}^{-1} ;{ }^{1} \mathrm{H}$ NMR (400 MHz, CDCl 3$): \delta 5.83$ $(\mathrm{d}, J=6.1 \mathrm{~Hz}, 1 \mathrm{H}), 5.79-5.74(\mathrm{~m}, 1 \mathrm{H}), 4.51(\mathrm{q}, J=3.2 \mathrm{~Hz}, 1 \mathrm{H}), 4.42-4.34(\mathrm{~m}, 1 \mathrm{H}), 2.58-2.38(\mathrm{~m}$, 2H), $2.31(\mathrm{~d}, J=11.6 \mathrm{~Hz}, 1 \mathrm{H}), 2.22-2.10(\mathrm{~m}, 2 \mathrm{H}), 2.04(\mathrm{~d}, J=13.4 \mathrm{~Hz}, 1 \mathrm{H}), 1.95-1.74(\mathrm{~m}, 3 \mathrm{H}), 1.74$ $-1.63(\mathrm{~m}, 2 \mathrm{H}), 0.87$ (s, 3H) ppm; ${ }^{13} \mathrm{C} \mathrm{NMR}\left(101 \mathrm{MHz}, \mathrm{CDCl}_{3}\right) \delta 172.9,136.0,129.8,85.0,78.1,51.1$, 47.7, 30.5, 29.5, 27.2, 26.6, 22.1, 11.2 ppm; HRMS (ESI-TOF, $m / z$ ): calcd for $\mathrm{C}_{13} \mathrm{H}_{18} \mathrm{O}_{3} \mathrm{Na}[\mathrm{M}+\mathrm{Na}]^{+}$ 245.1148 , found 245.1148 .

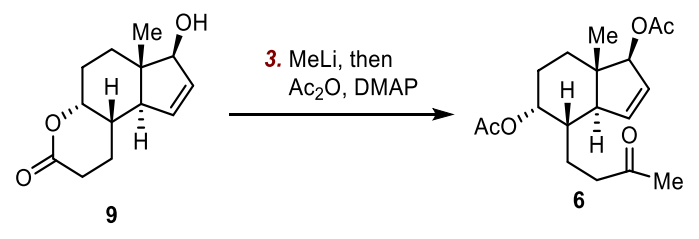

To a solution of 9 (200.0 mg, $0.9 \mathrm{mmol}, 1.0$ equiv) in $90 \mathrm{~mL}$ dry THF was added MeLi (1.6 $\mathrm{M}$ in $\mathrm{Et}_{2} \mathrm{O}$, $1.69 \mathrm{~mL}, 2.7 \mathrm{mmol}, 3.0$ equiv) at $-78^{\circ} \mathrm{C}$ under argon. After $15 \mathrm{~min}$, the reaction was completed and $\mathrm{Ac}_{2} \mathrm{O}$ $(0.17 \mathrm{~mL}, 1.8 \mathrm{mmol}, 2.0$ equiv) was added. The reaction mixture was warmed to room temperature and concentrated in vacuo. DMF (18 mL), DMAP (33.0 mg, 0.27 mmol, 0.3 equiv), DIPEA (3.00 mL, 18.0 mmol, 20.0 equiv), $\mathrm{Ac}_{2} \mathrm{O}$ ( $0.85 \mathrm{~mL}, 9.0 \mathrm{mmol}, 10.0$ equiv) was then added. The mixture was stirred at 60 ${ }^{\circ} \mathrm{C}$ for $5 \mathrm{~h}$ before quenched with saturated aqueous $\mathrm{Na}_{2} \mathrm{CO}_{3}$ solution and the aqueous phase was extracted with EtOAc $(3 \times 100 \mathrm{~mL})$. The combined organic layers were washed with brine $(3 \times 50 \mathrm{~mL})$, dried over $\mathrm{Na}_{2} \mathrm{SO}_{4}$, and concentrated in vacuo. The crude residue was purified by flash chromatography $\left(\mathrm{SiO}_{2}\right.$, DCM:EtOAc = 15:1) to afford compound $6(244.0 \mathrm{mg}, 84 \%)$.

Alternative procedure on large scale: To a solution of $9(6.3 \mathrm{~g}, 28.3 \mathrm{mmol}, 1.0$ equiv) in $567 \mathrm{~mL}$ dry THF was added MeLi (1.6 $\mathrm{M}$ in $\mathrm{Et}_{2} \mathrm{O}, 53.0 \mathrm{~mL}, 84.8 \mathrm{mmol}, 3.0$ equiv) at $-78^{\circ} \mathrm{C}$ under argon. After 20 min, the reaction mixture was quenched with $500 \mathrm{~mL}$ saturated aq. $\mathrm{NaHCO}_{3}$ and the aqueous phase was extracted with EtOAc $(4 \times 350 \mathrm{~mL})$. The combined organic layers were dried over $\mathrm{Na}_{2} \mathrm{SO}_{4}$, and 
concentrated in vacuo. The crude residue was dissolved in $280 \mathrm{~mL}$ DCM and DMAP (1.0 g, $8.5 \mathrm{mmol}$, 0.3 equiv), $\mathrm{Et}_{3} \mathrm{~N}$ (39.7 mL, $283.0 \mathrm{mmol}, 10.0$ equiv), $\mathrm{Ac}_{2} \mathrm{O}$ (16.0 mL, $170.0 \mathrm{mmol}, 6.0$ equiv) were added. After carried out at $40{ }^{\circ} \mathrm{C}$ for 4-5 h, the reaction was quenched with $300 \mathrm{~mL}$ saturated aq. $\mathrm{NaHCO}_{3}$ and the aqueous phase was extracted with DCM $(3 \times 200 \mathrm{~mL})$. The combined organic layers were dried over $\mathrm{Na}_{2} \mathrm{SO}_{4}$, and concentrated in vacuo. The crude residue was purified by flash chromatography $\left(\mathrm{SiO}_{2}\right.$, PE:EtOAc $=5: 1)$ to afford compound $6(8.7 \mathrm{~g}, 95 \%)$.

Compound 6: $R_{f}=0.85$ (silica gel, 9:1 DCM:EtOAc); $[\alpha]_{\mathrm{D}}^{24}-73.3\left(c 1.67, \mathrm{CHCl}_{3}\right) ; \mathrm{mp} 77.3{ }^{\circ} \mathrm{C}$; IR $(\mathrm{KBr})$ 2984, 2954, 1728, 1708, 1371, 1251, 1037, $1021 \mathrm{~cm}^{-1} ;{ }^{1} \mathrm{H}$ NMR (400 MHz, $\left.\mathrm{CDCl}_{3}\right) \delta 6.05-5.97(\mathrm{~m}, 1 \mathrm{H})$, $5.67(\mathrm{ddd}, J=5.9,3.4,1.4 \mathrm{~Hz}, 1 \mathrm{H}), 5.38-5.33(\mathrm{~m}, 1 \mathrm{H}), 5.08(\mathrm{q}, J=2.5 \mathrm{~Hz}, 1 \mathrm{H}), 2.61-2.46(\mathrm{~m}, 2 \mathrm{H})$, $2.37(\mathrm{ddd}, J=17.4,9.2,5.9 \mathrm{~Hz}, 1 \mathrm{H}), 2.12(\mathrm{~s}, 3 \mathrm{H}), 2.08$ (s, 3H), $2.04(\mathrm{~s}, 3 \mathrm{H}), 1.83-1.68(\mathrm{~m}, 5 \mathrm{H}), 1.62-$ $1.55(\mathrm{~m}, 1 \mathrm{H}), 1.46-1.35(\mathrm{~m}, 1 \mathrm{H}), 0.84(\mathrm{~s}, 3 \mathrm{H}) \mathrm{ppm} ;{ }^{13} \mathrm{C} \mathrm{NMR}\left(101 \mathrm{MHz}, \mathrm{CDCl}_{3}\right) \delta 208.5,171.1,170.7$ 132.7, 131.3, 86.2 , 69.5, 50.9, 49.8, 40.6, 36.8, 30.7, 30.2, 27.1, 22.6, 21.3, 21.2, 12.5 ppm; HRMS (ESITOF, $m / z)$ : calcd for $\mathrm{C}_{18} \mathrm{H}_{27} \mathrm{O}_{5}[\mathrm{M}+\mathrm{H}]^{+}$323.1853, found 323.1852.

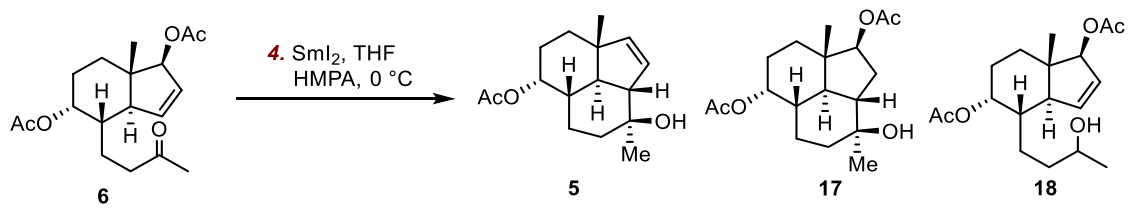

Preparation of SmI $\mathbf{S}^{1,2}$ : A $500 \mathrm{~mL}$ Schlenk flask containing a stir bar was charged with freshly ground Samarium powder (5.4 g, $35.7 \mathrm{mmol}, 1.4$ equiv). The system was flame-dried under high vacuum for 5 min, cooled to ambient temperature and the atmosphere was exchanged three times for argon. Subsequently, anhydrous degassed THF (250 mL) and distilled $\mathrm{CH}_{2} \mathrm{I}_{2}(2.0 \mathrm{~mL}, 25 \mathrm{mmol}, 1.0$ equiv) were added to the flask. The suspension was stirred for 3 min and the flask was cautiously and briefly (2 s) placed under partial high vacuum, then purged with argon. This process was repeated two additional times to remove the ethylene gas. The resulting heterogeneous suspension was rapidly (>900 rpm) stirred at room temperature for $1 \mathrm{~h}$ and the system was cautiously and briefly placed under high vacuum, then purged with argon for three times every one hour. After $3.5 \mathrm{~h}$, stirring was halted and the mixture was allowed to settle for $15 \mathrm{~min}$ prior to use.

A $500 \mathrm{~mL}$ dry round bottom flask equipped with a stir bar was charged with compound 6 (1.0 g, 3.1 mmol, 1.0 equiv). The atmosphere was exchanged three times for argon. Subsequently, the flask was charged with anhydrous THF (62 mL) and HMPA (13.5 mL, $77.5 \mathrm{mmol}, 25.0$ equiv). The reaction system was degassed by bubbling argon though the solution for $15 \mathrm{~min}$ at $0{ }^{\circ} \mathrm{C}$. Fresh prepared $\mathrm{SmI}_{2}(170.5 \mathrm{~mL}, 170.5$ 
mmol, 5.5 equiv) was added and the mixture was stirred at $0{ }^{\circ} \mathrm{C}$ for $3 \mathrm{~h}$. Then the reaction was quenched with saturated aqueous $\mathrm{NH}_{4} \mathrm{Cl}$ solution $(250 \mathrm{~mL})$ and extracted with EtOAc $(3 \times 250 \mathrm{~mL})$. The combined organic layers were washed with $2 \mathrm{~N} \mathrm{HCl}(2 \times 150 \mathrm{~mL})$ and brine, dried over $\mathrm{Na}_{2} \mathrm{SO}_{4}$, and concentrated in vacuo. The crude residue was purified by flash chromatography $\left(\mathrm{SiO}_{2}, \mathrm{PE}: \mathrm{EtOAc}=7: 1 \rightarrow 5: 1 \rightarrow 4: 1 \rightarrow 3: 1\right)$ to afford compound 5 (423.5 $\mathrm{mg}, 52 \%)$, compound 17 (314.3 mg, 31\%) and compound 18 (110.3 $\mathrm{mg}$, $11 \%)$.

Compound 5: white solid; $R_{f}=0.58$ (silica gel, 2:1 PE:EtOAc); $[\alpha]_{\mathrm{D}}^{24}+30.3\left(c 1.50, \mathrm{CHCl}_{3}\right)$; mp 107.6 ${ }^{\circ} \mathrm{C}$; IR (KBr) 3333, 2954, 2928, 2857, 1737, 1372, 1259, 1239, $1029 \mathrm{~cm}^{-1} ;{ }^{1} \mathrm{H}$ NMR (400 MHz, $\mathrm{CDCl}_{3}$ ) $\delta 6.02(\mathrm{dd}, J=5.8,2.4 \mathrm{~Hz}, 1 \mathrm{H}), 5.95(\mathrm{dd}, J=5.8,1.3 \mathrm{~Hz}, 1 \mathrm{H}), 5.06(\mathrm{q}, J=2.9 \mathrm{~Hz}, 1 \mathrm{H}), 2.21(\mathrm{~d}, J=12.5$ $\mathrm{Hz}, 1 \mathrm{H}), 2.05$ (s, 3H), $1.82-1.74(\mathrm{~m}, 2 \mathrm{H}), 1.72-1.64(\mathrm{~m}, 2 \mathrm{H}), 1.62-1.55(\mathrm{~m}, 3 \mathrm{H}), 1.41(\mathrm{td}, J=13.3$, $4.5 \mathrm{~Hz}, 2 \mathrm{H}), 1.31-1.21(\mathrm{~m}, 1 \mathrm{H}), 1.16(\mathrm{~s}, 3 \mathrm{H}), 0.86(\mathrm{~s}, 3 \mathrm{H}) \mathrm{ppm} ;{ }^{13} \mathrm{C} \mathrm{NMR}\left(101 \mathrm{MHz}, \mathrm{CDCl}_{3}\right) \delta 170.7$, $145.2,130.7,72.6,70.6,54.6,50.3,44.3,41.3,35.3,31.2,27.5,25.0,21.3,21.0,15.0$ ppm; HRMS (EITOF, $m / z$ ): calcd for $\mathrm{C}_{16} \mathrm{H}_{24} \mathrm{O}_{3}[\mathrm{M}]^{+} 264.1725$, found 264.1734 .

Compound 17: colorless oil; $R_{f}=0.23$ (silica gel, 2:1 PE:EtOAc); $[\alpha]_{\mathrm{D}}^{26}+6.1\left(c 1.34, \mathrm{CHCl}_{3}\right)$; IR $(\mathrm{KBr})$ 3445, 2941, 1735, 1437, 1373, 1243, $1032 \mathrm{~cm}^{-1} ;{ }^{1} \mathrm{H}$ NMR (400 MHz, CDCl $) \delta 5.00$ (q, $\left.J=2.8 \mathrm{~Hz}, 1 \mathrm{H}\right)$, $4.69(\mathrm{dd}, J=9.8,6.0 \mathrm{~Hz}, 1 \mathrm{H}), 2.14(\mathrm{ddd}, J=14.0,9.5,8.5 \mathrm{~Hz}, 1 \mathrm{H}), 2.04$ (s, 3H), $2.03(\mathrm{~s}, 3 \mathrm{H}), 1.84-1.75$ (m, 2H), $1.75-1.64(\mathrm{~m}, 2 \mathrm{H}), 1.64-1.52(\mathrm{~m}, 5 \mathrm{H}), 1.46(\mathrm{dd}, J=13.7,4.1 \mathrm{~Hz}, 1 \mathrm{H}), 1.38(\mathrm{td}, J=13.3,3.2$ $\mathrm{Hz}, 1 \mathrm{H}), 1.27-1.16(\mathrm{~m}, 1 \mathrm{H}), 1.16(\mathrm{~s}, 3 \mathrm{H}), 1.14(\mathrm{t}, J=13.1 \mathrm{~Hz}, 1 \mathrm{H}), 0.85(\mathrm{~s}, 3 \mathrm{H}) \mathrm{ppm} ;{ }^{13} \mathrm{C}$ NMR $(101$ $\left.\mathrm{MHz}, \mathrm{CDCl}_{3}\right) \delta 171.3,170.7,82.1,73.0,70.4,47.3,45.4,42.2,41.8,37.2,32.7,29.5,27.2,25.4,21.3$, 21.2, 20.1, 12.3 ppm; HRMS (ESI-TOF, $m / z$ ): calcd for $\mathrm{C}_{18} \mathrm{H}_{28} \mathrm{O}_{5} \mathrm{Na}[\mathrm{M}+\mathrm{Na}]^{+} 347.1829$, found 347.1830. Compound 18 (as a mixture of diastereomers, $d r 1: 1$ ): colorless oil; $R_{f}=0.40$ (silica gel, 2:1 PE:EtOAc); IR (KBr) 3455, 2940, 2863, 1735, 1455, 1437, 1373, 1243, $1022 \mathrm{~cm}^{-1} ;{ }^{1} \mathrm{H}$ NMR (400 MHz, $\left.\mathrm{CDCl}_{3}\right) \delta$ $6.05-5.98(\mathrm{~m}, 1 \mathrm{H}), 5.71-5.64(\mathrm{~m}, 1 \mathrm{H}), 5.37(\mathrm{~s}, 1 \mathrm{H}), 5.13(\mathrm{~s}, 1 \mathrm{H}), 3.84-3.68(\mathrm{~m}, 1 \mathrm{H}), 2.52(\mathrm{~d}, J=12.2$ Hz, 1H), 2.09 (s, 3H), 2.05 (s, 3H), $1.85-1.69(\mathrm{~m}, 4 \mathrm{H}), 1.66-1.56(\mathrm{~m}, 3 \mathrm{H}), 1.54-1.46(\mathrm{~m}, 1 \mathrm{H}), 1.43-$ $1.31(\mathrm{~m}, 2 \mathrm{H}), 1.19(\mathrm{~d}, J=7.6 \mathrm{~Hz}, 1.5 \mathrm{H}), 1.17(\mathrm{~d}, J=7.6 \mathrm{~Hz}, 1.5 \mathrm{H}), 0.87(\mathrm{~s}, 3 \mathrm{H}) \mathrm{ppm} ;{ }^{13} \mathrm{C}$ NMR $(101$ $\left.\mathrm{MHz}, \mathrm{CDCl}_{3}\right) \delta 171.3,171.2,170.9,170.7,132.97,132.95,131.2,131.1,86.4,70.2,70.0,68.4,68.1,50.9$, 50.0, 49.9, 37.6, 37.3, 36.5, 36.2, 30.8, 27.1, 25.2, 24.9, 23.7, 23.6, 21.4, 21.3, 21.2, 12.6, 12.5 ppm; HRMS (ESI-TOF, $m / z$ ): calcd for $\mathrm{C}_{18} \mathrm{H}_{29} \mathrm{O}_{5}[\mathrm{M}+\mathrm{H}]^{+} 325.2010$, found 325.2007. 


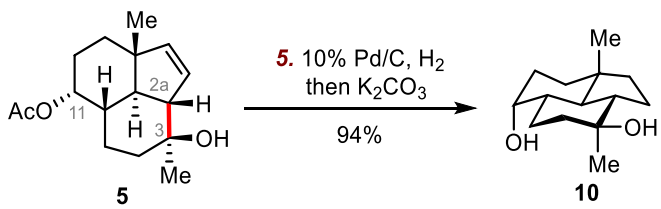

A mixture of compound $5(865.0 \mathrm{mg}, 3.3 \mathrm{mmol}, 1.0$ equiv) and $10 \% \mathrm{Pd} / \mathrm{C}(86.0 \mathrm{mg}, 10 \% \mathrm{w} / \mathrm{w})$ in $25 \mathrm{~mL}$ $\mathrm{MeOH}$ was stirred at room temperature under $1 \mathrm{~atm} \mathrm{H}_{2}$. The reaction was monitored by ${ }^{1} \mathrm{H}$ NMR until complete conversion of compound 5 was observed. After $23 \mathrm{~h}, \mathrm{~K}_{2} \mathrm{CO}_{3}(1.4 \mathrm{~g}, 13.2 \mathrm{mmol}, 4.0$ equiv) was added. Then the reaction was refluxed for $5 \mathrm{~h}$, quenched with water and extracted with EtOAc $(3 \times 5 \mathrm{~mL})$. The combined organic layers were washed with brine, dried over $\mathrm{Na}_{2} \mathrm{SO}_{4}$, and concentrated in vacuo. The crude residue was purified by flash chromatography $\left(\mathrm{SiO}_{2}, \mathrm{PE}: \mathrm{EtOAc}=1: 1\right)$ to afford compound $\mathbf{1 0}$ (690.5 mg, 94\%).

Compound 10: white solid; $R_{f}=0.40$ (silica gel, 1:1 PE:EtOAc); $[\alpha]_{\mathrm{D}}^{22}=+14.3\left(c 0.43, \mathrm{CHCl}_{3}\right) ; \mathrm{mp}$ $162.8^{\circ} \mathrm{C}$; IR (KBr) 3395, 2995, 2930, 2871, 1458, 1387, 1134, $1062 \mathrm{~cm}^{-1} ;{ }^{1} \mathrm{H}$ NMR (400 MHz, $\mathrm{CDCl}_{3}$ ) $\delta 3.84(\mathrm{q}, J=2.8 \mathrm{~Hz}, 1 \mathrm{H}), 1.79-1.67(\mathrm{~m}, 4 \mathrm{H}), 1.64-1.57(\mathrm{~m}, 1 \mathrm{H}), 1.56-1.47(\mathrm{~m}, 4 \mathrm{H}), 1.46-1.40(\mathrm{~m}$, 2H), $1.39-1.30(\mathrm{~m}, 2 \mathrm{H}), 1.34-1.22(\mathrm{~m}, 1 \mathrm{H}), 1.17(\mathrm{~s}, 3 \mathrm{H}), 1.02(\mathrm{t}, J=12.6 \mathrm{~Hz}, 1 \mathrm{H}), 0.77(\mathrm{~s}, 3 \mathrm{H}) \mathrm{ppm}$; ${ }^{13} \mathrm{C}$ NMR $\left(101 \mathrm{MHz}, \mathrm{CDCl}_{3}\right) \delta$ 74.0, 68.2, 48.9, 47.3, 42.6, 39.7, 39.3, 39.0, 33.7, 30.6, 25.6, 21.2, 20.2, 17.6 ppm; HRMS (EI-TOF, $m / z$ ): calcd for $\mathrm{C}_{14} \mathrm{H}_{24} \mathrm{O}_{2}[\mathrm{M}]^{+}$224.1771, found 224.1776.

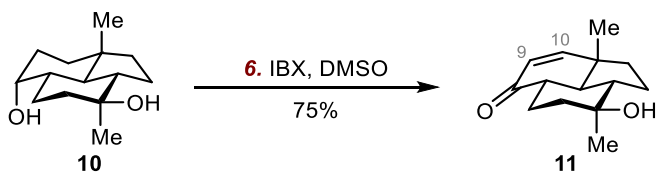

To a solution of compound $\mathbf{1 0}(1.1 \mathrm{~g}, 4.7 \mathrm{mmol}, 1.0$ equiv) in $47 \mathrm{~mL}$ DMSO were added PTSA (266.0 mg, 1.4 mmol, 0.3 equiv) and IBX ${ }^{3}\left(5.7 \mathrm{~g}, 20.4 \mathrm{mmol}, 4.4\right.$ equiv). The solution was heated at $100{ }^{\circ} \mathrm{C}$ for $5 \mathrm{~h}$ and quenched with water $(470 \mathrm{~mL})$. The aqueous layer was extracted with EtOAc $(3 \times 200 \mathrm{~mL})$ and the combined organic layers were washed with brine $(200 \mathrm{~mL})$, dried over $\mathrm{NaSO}_{4}$, concentrated in vacuo. The crude residue was purified by flash chromatography (alkaline alumina oxide, PE:EtOAc $=1.5: 1$ ) to afford compound $11(777.8 \mathrm{mg}, 75 \%)$.

Compound 11: white solid; $R_{f}=0.58$ (silica gel, 1:1 PE:EtOAc); $[\alpha]_{\mathrm{D}}^{29}-12.5\left(c 1.01, \mathrm{CHCl}_{3}\right.$ ); mp 139.4 ${ }^{\circ} \mathrm{C}$; IR (KBr) 3494, 2970, 2935, 2872, 1660, 1460, 1383, 1156, $1114 \mathrm{~cm}^{-1} ;{ }^{1} \mathrm{H}$ NMR (400 MHz, $\left.\mathrm{CDCl}_{3}\right) \delta$ $7.11(\mathrm{~d}, J=9.7 \mathrm{~Hz}, 1 \mathrm{H}), 5.79(\mathrm{~d}, J=9.8 \mathrm{~Hz}, 1 \mathrm{H}), 2.25-2.12(\mathrm{~m}, 2 \mathrm{H}), 1.94-1.78(\mathrm{~m}, 3 \mathrm{H}), 1.71-1.61$ $(\mathrm{m}, 2 \mathrm{H}), 1.59-1.54(\mathrm{~m}, 1 \mathrm{H}), 1.51-1.38(\mathrm{~m}, 2 \mathrm{H}), 1.35-1.23(\mathrm{~m}, 1 \mathrm{H}), 1.18(\mathrm{~s}, 3 \mathrm{H}), 1.00(\mathrm{~s}, 3 \mathrm{H}) \mathrm{ppm}$; 
${ }^{13} \mathrm{C}$ NMR $\left(101 \mathrm{MHz} \mathrm{CDCl}_{3}\right) \delta 201.6,158.4,129.1,73.2,52.8,48.4,44.7,42.3,41.9,35.2,24.6,21.7$, 20.2, 19.6 ppm; HRMS (EI-TOF, $m / z$ ): calcd for $\mathrm{C}_{14} \mathrm{H}_{20} \mathrm{O}_{2}[\mathrm{M}]^{+}$220.1463, found 220.1468.

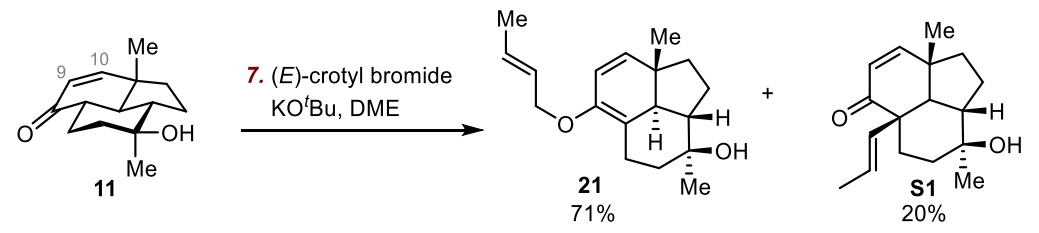

To a dry round bottom flask compound $11\left(337.0 \mathrm{mg}, 1.53 \mathrm{mmol}, 1.0\right.$ equiv) and ${ }^{t} \mathrm{BuOK}(772.0 \mathrm{mg}, 6.89$ mmol, 4.5 equiv) were added. The flask was cooled to $-60{ }^{\circ} \mathrm{C}$ before adding $45 \mathrm{~mL}$ DME. The reaction mixture was stirred at this temperature for $15 \mathrm{~min}$, and then $(E)$-crotyl bromide $(0.59 \mathrm{~mL}, 4.59 \mathrm{mmol}, 3.0$ equiv) was added. After stirring for another $2 \mathrm{~h}$, the reaction mixture was quenched with water $(50 \mathrm{~mL})$ and extracted with EtOAc $(3 \times 50 \mathrm{~mL})$. The combined organic layers were washed with brine $(50 \mathrm{~mL})$, dried over $\mathrm{Na}_{2} \mathrm{SO}_{4}$, and concentrated in vacuo. The crude residue was purified by flash chromatography (alkaline alumina oxide, PE:EtOAc $\left.=4: 1\left(+2 \% \mathrm{Et}_{3} \mathrm{~N}\right) \rightarrow 2: 1\left(+2 \% \mathrm{Et}_{3} \mathrm{~N}\right)\right)$ to afford compound $21(298.0$ $\mathrm{mg}, 71 \%)$ and $22(83.9 \mathrm{mg}, 20 \%)$.

Compound 21: white solid; $R_{f}=0.68$ (silica gel, 2:1 PE:EtOAc); $[\alpha]_{\mathrm{D}}^{25}-87.2$ (c 1.68, $\left.\mathrm{CHCl}_{3}\right)$; IR $(\mathrm{KBr})$ 3395, 3020, 2931, 2868, 1639, 1455, 1390, 1372, 1203, $1136 \mathrm{~cm}^{-1} ;{ }^{1} \mathrm{H} \mathrm{NMR}\left(400 \mathrm{MHz}, \mathrm{CDCl}_{3}\right): \delta 6.00$ $(\mathrm{d}, J=9.7 \mathrm{~Hz}, 1 \mathrm{H}), 5.75(\mathrm{~d}, J=9.8 \mathrm{~Hz}, 1 \mathrm{H}), 5.80-5.66(\mathrm{~m}, 1 \mathrm{H}), 5.65-5.55(\mathrm{~m}, 1 \mathrm{H}), 4.23(\mathrm{dd}, J=11.9$, $5.9 \mathrm{~Hz}, 1 \mathrm{H}), 4.10(\mathrm{dd}, J=11.9,6.4 \mathrm{~Hz}, 1 \mathrm{H}), 2.66(\mathrm{ddt}, J=18.1,5.7,2.7 \mathrm{~Hz}, 1 \mathrm{H}), 2.28-2.11(\mathrm{~m}, 1 \mathrm{H})$, $2.02-1.89(\mathrm{~m}, 2 \mathrm{H}), 1.88-1.78(\mathrm{~m}, 1 \mathrm{H}), 1.78-1.72(\mathrm{~m}, 1 \mathrm{H}), 1.70(\mathrm{dd}, J=6.3,1.3 \mathrm{~Hz}, 3 \mathrm{H}), 1.68-1.62$ $(\mathrm{m}, 2 \mathrm{H}), 1.62-1.52(\mathrm{~m}, 2 \mathrm{H}), 1.50-1.44(\mathrm{~m}, 1 \mathrm{H}), 1.17(\mathrm{~s}, 3 \mathrm{H}), 0.80(\mathrm{~s}, 3 \mathrm{H}) \mathrm{ppm} ;{ }^{13} \mathrm{C} \mathrm{NMR}(101 \mathrm{MHz}$, $\left.\mathrm{CDCl}_{3}\right): \delta 146.3,137.7,130.3,127.7,123.8,117.6,74.1,71.4,52.3,45.6,41.7,41.1,33.6,23.1,23.0$, 19.9, 18.6, 18.2 ppm; HRMS (ESI-TOF, $m / z$ ): calcd for $\mathrm{C}_{18} \mathrm{H}_{27} \mathrm{O}_{2}[\mathrm{M}+\mathrm{H}]^{+}$275.2006, found 275.2004.

Compound S1: colorless oil; $R_{f}=0.51$ (silica gel, 2:1 PE:EtOAc); $[\alpha]_{\mathrm{D}}^{25}+23.4\left(\right.$ c 2.00, $\left.\mathrm{CHCl}_{3}\right) ; \mathrm{IR}(\mathrm{KBr})$ 3410, 2961, 2927, 2873, 1664, 1467, 1381, 1261, 1108, $1031 \mathrm{~cm}^{-1} ;{ }^{1} \mathrm{H} \mathrm{NMR}\left(400 \mathrm{MHz}, \mathrm{CDCl}_{3}\right): \delta 7.03$ $(\mathrm{d}, J=9.8 \mathrm{~Hz}, 1 \mathrm{H}), 5.80(\mathrm{~d}, J=9.8 \mathrm{~Hz}, 1 \mathrm{H}), 5.51-5.27(\mathrm{~m}, 2 \mathrm{H}), 2.32(\mathrm{dd}, J=14.4,8.8 \mathrm{~Hz}, 1 \mathrm{H}), 2.28-$ $2.23(\mathrm{~m}, 1 \mathrm{H}), 2.17(\mathrm{ddd}, J=14.1,10.0,6.5 \mathrm{~Hz}, 1 \mathrm{H}), 2.06(\mathrm{dt}, J=14.2,3.4 \mathrm{~Hz}, 1 \mathrm{H}), 1.95-1.81(\mathrm{~m}, 1 \mathrm{H})$, $1.76-1.68(\mathrm{~m}, 1 \mathrm{H}), 1.65(\mathrm{~d}, J=6.1 \mathrm{~Hz}, 3 \mathrm{H}), 1.62-1.53(\mathrm{~m}, 4 \mathrm{H}), 1.49-1.41(\mathrm{~m}, 2 \mathrm{H}), 1.20(\mathrm{~s}, 3 \mathrm{H})$, 1.13 (s, 3H) ppm; ${ }^{13} \mathrm{C}$ NMR (101 MHz, $\left.\mathrm{CDCl}_{3}\right): \delta 204.1,157.0,128.1,128.1,125.9,73.8,54.6,48.2$, 43.7, 42.7, 39.0, 38.3 31.2, 28.6, 21.4, 21.1, 20.9, 18.2 ppm; HRMS (ESI-TOF, $m / z$ ): calcd for $\mathrm{C}_{18} \mathrm{H}_{27} \mathrm{O}_{2}$ $[\mathrm{M}+\mathrm{H}]^{+}$275.2006, found 275.2004. 


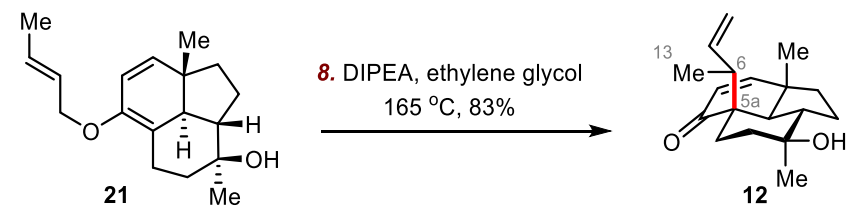

To a round-bottom flask equipped with a stir bar was added compound 21 (514.0 mg, $1.88 \mathrm{mmol}, 1.0$ equiv). After the flask was evacuated and backfilled with argon three times, $50 \mathrm{~mL}$ ethylene glycol and DIPEA ( $0.93 \mathrm{~mL}, 5.64 \mathrm{mmol}, 3.0$ equiv) were added. The reaction mixture was heated at $165^{\circ} \mathrm{C}$ for $2 \mathrm{~h}$, quenched with water $(50 \mathrm{~mL})$ and extracted with EtOAc $(3 \times 75 \mathrm{~mL})$. The combined organic layers were washed with brine $(50 \mathrm{~mL})$, dried over anhydrous $\mathrm{Na}_{2} \mathrm{SO}_{4}$, and concentrated in vacuo. The crude residue was purified by flash chromatography (alkaline alumina oxide, PE:EtOAc $=3: 1 \rightarrow 1.5: 1$ ) to afford compound 12 (426.6 mg, 83\%).

Compound 12: Colorless oil; $R_{f}=0.50$ (silica gel, 2:1 PE:EtOAc); $[\alpha]_{\mathrm{D}}^{25}-1.3\left(c 0.44, \mathrm{CHCl}_{3}\right)$; IR (KBr) 3446, 2960, 2872, 1673, 1457, 1381, $1105 \mathrm{~cm}^{-1} ;{ }^{1} \mathrm{H}$ NMR (400 MHz, $\left.\mathrm{CDCl}_{3}\right): \delta 6.93$ (d, J=9.8 Hz, 1H), $5.87-5.77(\mathrm{~m}, 1 \mathrm{H}), 5.78$ (d, $J=9.9 \mathrm{~Hz}, 1 \mathrm{H}), 4.98$ (d, $J=10.0 \mathrm{~Hz}, 1 \mathrm{H}), 4.89$ (d, $J=17.1 \mathrm{~Hz}, 1 \mathrm{H}), 2.81$ (quint, $J=7.1 \mathrm{~Hz}, 1 \mathrm{H}$ ), 2.45 (ddd, $J=14.4,10.1,6.1 \mathrm{~Hz}, 1 \mathrm{H}), 2.05$ (dt, $J=15.1,3.7 \mathrm{~Hz}, 1 \mathrm{H}$ ), $1.96-1.85$ (m, 1H), 1.79 (dd, $J=14.0,4.0 \mathrm{~Hz}, 1 \mathrm{H}), 1.74-1.63$ (m, 3H), 1.47 (td, $J=15.0,4.5 \mathrm{~Hz}, 1 \mathrm{H}), 1.42-1.34$ $(\mathrm{m}, 2 \mathrm{H}), 1.30(\mathrm{~d}, J=7.1 \mathrm{~Hz}, 3 \mathrm{H}), 1.27(\mathrm{~s}, 3 \mathrm{H}), 1.17(\mathrm{~s}, 3 \mathrm{H}) \mathrm{ppm} ;{ }^{13} \mathrm{C}$ NMR (101 MHz, $\left.\mathrm{CDCl}_{3}\right): \delta 204.4$, 155.5, 140.6, 127.6, 115.1, 73.8, 54.0, 51.6, 45.9, 43.0, 41.6, 39.9, 39.3, 26.4, 21.0, 20.1, 19.6, 18.5 ppm; HRMS (ESI-TOF, $m / z$ ): calcd for $\mathrm{C}_{18} \mathrm{H}_{27} \mathrm{O}_{2}[\mathrm{M}+\mathrm{H}]^{+}$275.2006, found 275.2006.

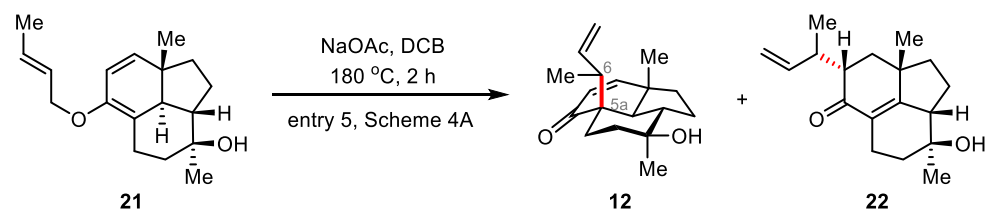

In a round-bottom flask equipped with a stir bar, compound 21 (28.0 $\mathrm{mg}, 0.1 \mathrm{mmol}, 1.0$ equiv) and $\mathrm{NaOAc}$ (24.6 mg, $0.3 \mathrm{mmol}, 3.0$ equiv) was added. After the flask was evacuated and backfilled with argon three times, $2 \mathrm{~mL} \mathrm{1,2-dichlorobenzene} \mathrm{was} \mathrm{added.} \mathrm{The} \mathrm{reaction} \mathrm{mixture} \mathrm{was} \mathrm{heated} \mathrm{at} 180{ }^{\circ} \mathrm{C}$ for $2 \mathrm{~h}$. After concentration in vacuo, the crude residue was purified by flash chromatography (alkaline alumina oxide, PE:EtOAc $=3: 1 \rightarrow 1.5: 1)$ to afford a mixture of compound $\mathbf{1 2}$ and $\mathbf{2 2}(19.3 \mathrm{mg}, 69 \%, \mathbf{1 2 : 2 2}=1: 0.8)$.

Compound 22: Colorless oil; $R_{f}=0.50$ (silica gel, 2:1 PE:EtOAc); IR (KBr) 3386, 2960, 2926, 2856, 1658, 1459, 1375, 1283, $1119 \mathrm{~cm}^{-1} ;{ }^{1} \mathrm{H}$ NMR $\left(400 \mathrm{MHz}, \mathrm{CDCl}_{3}\right): \delta 5.90-5.79(\mathrm{~m}, 0.5 \mathrm{H}), 5.69$ (ddd, $J=$ 17.1, 10.5, 6.4 Hz, 0.5H), $5.06-5.00(\mathrm{~m}, 1 \mathrm{H}), 5.00-4.93(\mathrm{~m}, 1 \mathrm{H}), 3.21-3.07(\mathrm{~m}, 1 \mathrm{H}), 2.91-2.81(\mathrm{~m}$, 1H), $2.59-2.39(\mathrm{~m}, 2 \mathrm{H}), 2.20-2.08(\mathrm{~m}, 1 \mathrm{H}), 2.07-1.97(\mathrm{~m}, 1 \mathrm{H}), 1.95-1.83(\mathrm{~m}, 1 \mathrm{H}), 1.83-1.74(\mathrm{~m}$, 
$3 \mathrm{H}), 1.68-1.58(\mathrm{~m}, 2 \mathrm{H}), 1.59-1.45(\mathrm{~m}, 1 \mathrm{H}), 1.41-1.31(\mathrm{~m}, 1 \mathrm{H}), 1.19(\mathrm{~s}, 3 \mathrm{H}), 1.07(\mathrm{~d}, J=7.1 \mathrm{~Hz}$, 1.5H), $1.01(\mathrm{~s}, 1.5 \mathrm{H}), 1.00(\mathrm{~s}, 1.5 \mathrm{H}), 0.89(\mathrm{~d}, J=6.9 \mathrm{~Hz}, 1.5 \mathrm{H}) \mathrm{ppm} ;{ }^{13} \mathrm{C} \mathrm{NMR}\left(101 \mathrm{MHz}, \mathrm{CDCl}_{3}\right) \delta$ 199.32, 199.29, 168.2, 168.1, 142.8, 140.7, 126.5, 126.4, 114.6, 113.8, 71.87, 71.87, 49.43, 49.37, 47.3, 46.3, 42.5, 42.4, 41.3, 41.2, 38.0, 37.9, 37.4, 35.3, 35.2, 22.7, 22.34, 22.30, 22.2, 22.1, 22.0, 20.5, 20.4, 17.0, 14.0 ppm; HRMS (ESI-TOF, $m / z$ ): calcd for $\mathrm{C}_{18} \mathrm{H}_{27} \mathrm{O}_{2}[\mathrm{M}+\mathrm{H}]^{+}$275.2006, found 275.2006.

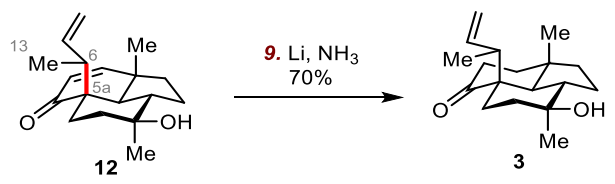

Ammonia $(10 \mathrm{~mL})$ was condensed into a three-neck round-bottom flask equipped with a stir bar at $-78{ }^{\circ} \mathrm{C}$. To this flask was added Li wire (16.2 mg, $2.33 \mathrm{mmol}, 2.5$ equiv) to obtain a dark-blue solution. A solution of compound 12 (254.0 mg, $0.93 \mathrm{mmol}, 1.0$ equiv) in THF (25 mL) was quickly added to the mixture. The blue color disapeared in a few seconds and the reaction mixture was allowed to warm to room temperature, and $\mathrm{NH}_{3}$ was allowed to evaporate. The residue was quenched by saturated aqueous $\mathrm{NH}_{4} \mathrm{Cl}$ solution $(10 \mathrm{~mL})$, taken up in $\mathrm{H}_{2} \mathrm{O}(20 \mathrm{~mL})$ and extracted with EtOAc $(3 \times 50 \mathrm{~mL})$. The combined organic layers were washed with brine $(50 \mathrm{~mL})$, dried over anhydrous $\mathrm{Na}_{2} \mathrm{SO}_{4}$, and concentrated in vacuo. The crude residue was purified by flash chromatography $\left(\mathrm{SiO}_{2}, \mathrm{PE}: \mathrm{EtOAc}=4: 1\right)$ to afford compound 3 (178.9 $\mathrm{mg}, 70 \%)$.

Compound 3: white solid; $R_{f}=0.55$ (silica gel, 2:1 PE:EtOAc); $[\alpha]_{\mathrm{D}}^{25} 5.6\left(c\right.$ 1.30, $\left.\mathrm{CHCl}_{3}\right) ; \mathrm{mp} 130.4{ }^{\circ} \mathrm{C}$; IR (KBr) 3401, 2958, 2863, 1707, 1461, 1384, 1238, $1136 \mathrm{~cm}^{-1} ;{ }^{1} \mathrm{H}$ NMR (400 MHz, $\left.\mathrm{CDCl}_{3}\right): \delta 5.67$ (ddd, $J=17.0,10.3,8.1 \mathrm{~Hz}, 1 \mathrm{H}), 4.96(\mathrm{~d}, J=17.2 \mathrm{~Hz}, 1 \mathrm{H}), 4.93(\mathrm{~d}, J=10.7 \mathrm{~Hz}, 1 \mathrm{H}), 3.18$ (quint, $J=7.3 \mathrm{~Hz}$, 1H), $2.83(\mathrm{td}, J=14.1,6.3 \mathrm{~Hz}, 1 \mathrm{H}), 2.48(\mathrm{ddd}, J=13.7,10.6,5.6 \mathrm{~Hz}, 1 \mathrm{H}), 2.13$ (ddd, $J=14.1,4.8,2.1$ $\mathrm{Hz}, 1 \mathrm{H}), 2.02(\mathrm{ddd}, J=13.2,6.3,2.2 \mathrm{~Hz}, 1 \mathrm{H}), 1.96-1.85(\mathrm{~m}, 1 \mathrm{H}), 1.85-1.75(\mathrm{~m}, 2 \mathrm{H}), 1.73-1.64(\mathrm{~m}$, 2H), $1.64-1.53(\mathrm{~m}, 3 \mathrm{H}), 1.36(\mathrm{~d}, J=7.1 \mathrm{~Hz}, 3 \mathrm{H}), 1.34(\mathrm{~s}, 3 \mathrm{H}), 1.22(\mathrm{~d}, J=13.8 \mathrm{~Hz}, 1 \mathrm{H}), 1.14(\mathrm{~s}, 3 \mathrm{H})$, 1.16 - $1.09(\mathrm{~m}, 1 \mathrm{H}) \mathrm{ppm} ;{ }^{13} \mathrm{C} \mathrm{NMR}\left(101 \mathrm{MHz}, \mathrm{CDCl}_{3}\right) \delta 215.7,140.5,114.6,74.0,59.3,55.5,45.5,42.6$, 41.2, 41.1, 40.9, 40.4 36.2, 26.6, 23.0, 20.2, 20.1, 19.8 ppm; HRMS (ESI-TOF, m/z): calcd for $\mathrm{C}_{18} \mathrm{H}_{29} \mathrm{O}_{2}$ $[\mathrm{M}+\mathrm{H}]^{+} 277.2162$, found 277.2160
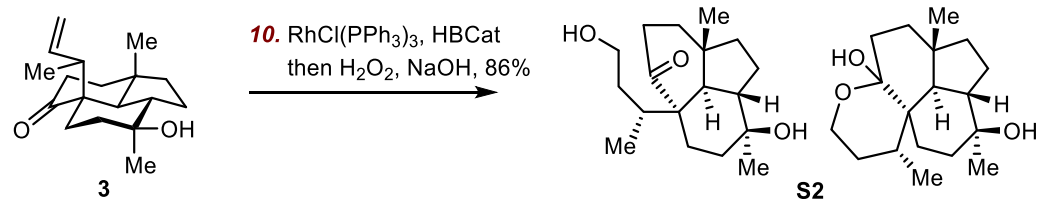

Preparation of the catalyst (partially oxidized $\left.\mathrm{RhCl}\left(\mathrm{PPh}_{3}\right)_{3}\right):{ }^{4}$ Oxygen was passed through a solution of 
$\mathrm{Rh}\left(\mathrm{PPh}_{3}\right)_{3} \mathrm{Cl}(5.0 \mathrm{mg}, 0.0055 \mathrm{mmol}, 5 \mathrm{~mol} \%)$ in $5 \mathrm{~mL}$ of DCM for $30 \mathrm{~min}$. The solvent was removed in vacuo and the catalyst was kept under high vacuum $(<0.1$ Torr) for at least 30 min prior to use.

To a solution of compound 3 (30.0 mg, $0.11 \mathrm{mmol}, 1.0$ equiv) and partially oxidized $\mathrm{RhCl}\left(\mathrm{PPh}_{3}\right)_{3}$ in $\mathrm{THF}$ $(6 \mathrm{~mL})$ was added dropwise catecholborane $(60 \mu \mathrm{L}, 0.40 \mathrm{mmol}, 3.6$ equiv) at $\mathrm{rt}$ under argon. After stirred at $\mathrm{rt}$ for $20 \mathrm{~h}$, the reaction mixture was cooled to $0{ }^{\circ} \mathrm{C}$ and $2.0 \mathrm{M} \mathrm{NaOH}$ solution $(6 \mathrm{~mL})$ and $30 \% \mathrm{H}_{2} \mathrm{O}_{2}(3$ $\mathrm{mL}$ ) were added successively. The resulting mixture was warmed to room temperature and allowed to stir for $2 \mathrm{~h}$ before it was quenched with saturated aq. $\mathrm{Na}_{2} \mathrm{SO}_{3}(10 \mathrm{~mL})$. The mixture was extracted with EtOAc $(5 \times 25 \mathrm{~mL})$, and the combined organic layers were washed with brine $(25 \mathrm{~mL})$, dried over anhydrous $\mathrm{MgSO}_{4}$, filtered, and concentrated in vacuo. The crude residue was purified by flash chromatography $\left(\mathrm{SiO}_{2}, \mathrm{PE}: \mathrm{EtOAc}=1.5: 1\right)$ to afford compound $\mathbf{S 2}(27.5 \mathrm{mg}, 86 \%)$ as a mixtue of primary alcohol and its corresponding hemiketal (ratio: $1: 1$ ).

Compound S2: colorless oil; $R_{f}=0.35$ (silica gel, 1:1 PE:EtOAc); IR (KBr) 3372, 2957, 2863, 1692, 1467, 1379, 1098, $1053 \mathrm{~cm}^{-1} ;{ }^{1} \mathrm{H} \mathrm{NMR}\left(400 \mathrm{MHz}, \mathrm{CDCl}_{3}\right): \delta 3.78$ (dd, J=9.7, $\left.2.3 \mathrm{~Hz}, 1 \mathrm{H}\right), 3.74-3.63$ $(\mathrm{m}, 0.5 \mathrm{H}), 3.60-3.48(\mathrm{~m}, 0.5 \mathrm{H}), 2.98(\mathrm{td}, J=14.1,6.5 \mathrm{~Hz}, 0.5 \mathrm{H}), 2.70-2.63(\mathrm{~m}, 1 \mathrm{H}), 2.63-2.45(\mathrm{~m}$, 1H), $2.29(\mathrm{ddd}, J=14.5,10.5,5.6 \mathrm{~Hz}, 0.5 \mathrm{H}), 2.12(\mathrm{ddd}, J=14.1,5.0,2.1 \mathrm{~Hz}, 0.5 \mathrm{H}), 2.03(\mathrm{ddd}, J=13.0$, 6.4, $2.3 \mathrm{~Hz}, 0.5 \mathrm{H}), 1.94-1.43(\mathrm{~m}, 15 \mathrm{H}), 1.33(\mathrm{~s}, 1.5 \mathrm{H}), 1.32(\mathrm{~d}, J=7.7 \mathrm{~Hz}, 1.5 \mathrm{H}), 1.22(\mathrm{~d}, J=6.4 \mathrm{~Hz}$ 1.5H), $1.21(\mathrm{~s}, 1.5 \mathrm{H}), 1.15(\mathrm{~s}, 1.5 \mathrm{H}), 1.09$ (s, 1.5H) ppm; $\left.{ }^{13} \mathrm{C} \mathrm{NMR} \mathrm{(101} \mathrm{MHz,} \mathrm{CDCl}_{3}\right) \delta 217.3,100.7$, 74.5, 74.0, 63.1, 60.5, 59.6, 56.3, 52.3, 45.9, 45.7, 45.1, 43.3, 42.4, 41.5, 41.4, 41.0, 40.5, 40.3, 36.9, 36.6, 35.9, 33.6, 32.7, 31.5, 28.9, 26.5, 23.0, 22.4, 22.12, 22.05, 20.3, 20.1, 19.88, 19.86, 19.5 ppm; HRMS (ESI-TOF, $m / z$ ): calcd for $\mathrm{C}_{18} \mathrm{H}_{30} \mathrm{O}_{3} \mathrm{Na}[\mathrm{M}+\mathrm{Na}]^{+}$317.2087, found 317.2087.

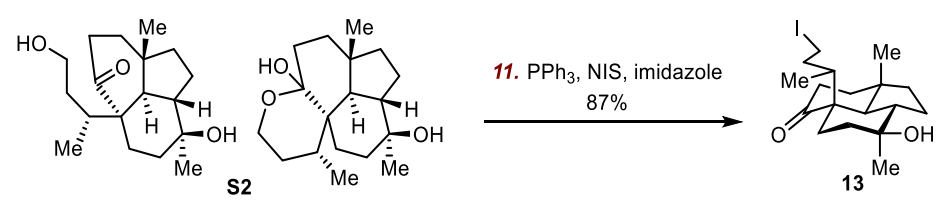

To a solution of $\mathbf{S 2}$ (10.0 mg, $0.034 \mathrm{mmol}, 1.0$ equiv), $\mathrm{PPh}_{3}(63.0 \mathrm{mg}, 0.24 \mathrm{mmol}, 7.0$ equiv) and imidazole (6.8 mg, $0.10 \mathrm{mmol}, 3.0$ equiv) in $3 \mathrm{~mL}$ DCM was added NIS (54.0 mg, $0.24 \mathrm{mmol}, 7.0$ equiv). The reaction was stirred for $40 \mathrm{~min}$ before it was quenched with saturated aq. $\mathrm{Na}_{2} \mathrm{~S}_{2} \mathrm{O}_{3}(5 \mathrm{~mL})$. The mixture was extracted with EtOAc $(3 \times 10 \mathrm{~mL})$, and the combined organic layers were washed with brine $(10 \mathrm{~mL})$, dried over anhydrous $\mathrm{MgSO}_{4}$, filtered, and concentrated in vacuo. The crude residue was purified by flash chromatography $\left(\mathrm{SiO}_{2}, \mathrm{PE}: \mathrm{EtOAc}=4: 1\right)$ to afford compound $13(11.9 \mathrm{mg}, 87 \%)$.

Compound 13: Colorless oil; $R_{f}=0.40$ (silica gel, 2:1 PE:EtOAc); $[\alpha]_{\mathrm{D}}^{25}+49.5\left(c 1.40, \mathrm{CHCl}_{3}\right) ; \mathrm{IR}(\mathrm{KBr})$ 
3391, 2930, 2861, 1699, 1465, 1381, 1261, 1185, $1100 \mathrm{~cm}^{-1} ;{ }^{1} \mathrm{H}$ NMR (400 MHz, $\mathrm{CDCl}_{3}$ ): $\delta 3.36$ (ddd, $J$ = 9.6, 5.6, 3.7 Hz, 1H), $3.09(\mathrm{ddd}, J=15.2,10.2,5.0 \mathrm{~Hz}, 1 \mathrm{H}), 3.02(\mathrm{td}, J=13.9,6.2 \mathrm{~Hz}, 1 \mathrm{H}), 2.68-2.60$ (m, 1H), $2.57(\mathrm{ddd}, J=13.8,10.5,5.7 \mathrm{~Hz}, 1 \mathrm{H}), 2.15(\mathrm{ddd}, J=14.0,4.9,2.1 \mathrm{~Hz}, 1 \mathrm{H}), 2.05(\mathrm{ddd}, J=13.1$, 6.3, 2.1 Hz, 1H), $1.91(\mathrm{dtd}, J=14.1,10.4,8.8 \mathrm{~Hz}, 1 \mathrm{H}), 1.79-1.47(\mathrm{~m}, 10 \mathrm{H}), 1.36(\mathrm{~s}, 3 \mathrm{H}), 1.26(\mathrm{~d}, J=$ $7.0 \mathrm{~Hz}, 3 \mathrm{H}), 1.21(\mathrm{t}, J=14.3 \mathrm{~Hz}, 1 \mathrm{H}), 1.14(\mathrm{~s}, 3 \mathrm{H}) \mathrm{ppm} ;{ }^{13} \mathrm{C} \mathrm{NMR}\left(101 \mathrm{MHz}, \mathrm{CDCl}_{3}\right) \delta 216.6,73.9,59.7$, 56.0, 45.7, 42.4 4 41.5, 40.8, 40.5, 36.4, 36.3, 36.1, 27.0, 23.0, 20.2 20.0, 17.9, 7.1 ppm; HRMS (ESI-TOF, $m / z)$ : calcd for $\mathrm{C}_{18} \mathrm{H}_{30} \mathrm{IO}_{2}[\mathrm{M}+\mathrm{H}]^{+} 405.1285$, found 405.1284 .

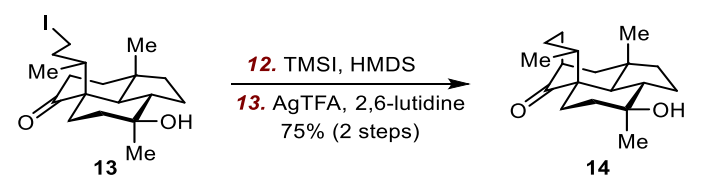

To a solution of compound $13(50.0 \mathrm{mg}, 0.12 \mathrm{mmol}, 1.0$ equiv) in $1 \mathrm{~mL}$ DCM, HMDS (0.18 mL, 0.87 mmol, 7.0 equiv) and TMSI ( $88 \mu \mathrm{L}, 0.62 \mathrm{mmol}, 5.0$ equiv) were added at rt. After stirred at $40^{\circ} \mathrm{C}$ for $2 \mathrm{~h}$, the reaction mixture was quenched with saturated aq. $\mathrm{NaHCO}_{3}$ solution $(5 \mathrm{~mL})$ and extracted with DCM $(3 \times 5 \mathrm{~mL})$. The combined organic layers were washed with brine $(5 \mathrm{~mL})$, dried over anhydrous $\mathrm{Na}_{2} \mathrm{SO}_{4}$, and concentrated in vacuo. The crude was dissolved in $5 \mathrm{~mL}$ THF and 2,6-lutidine $(29 \mu \mathrm{L}, 0.25 \mathrm{mmol}$, 2.0 equiv) and silver trifluoroacetate $(82.0 \mathrm{mg}, 0.37 \mathrm{mmol}, 3.0$ equiv) was added. The reaction mixture was stirred at room temperature for $3 \mathrm{~h}$ before PTSA (71.0 mg, $0.37 \mathrm{mmol}, 3.0$ equiv) was added. After additional $4 \mathrm{~h}$, the reaction was quenched with saturated aq. $\mathrm{NaHCO}_{3}$ solution $(10 \mathrm{~mL})$ and extracted with EtOAc $(3 \times 10 \mathrm{~mL})$. The combined organic layers were washed with brine $(10 \mathrm{~mL})$, dried over $\mathrm{Na}_{2} \mathrm{SO}_{4}$ and concentrated in vacuo. The crude residue was purified by flash chromatography $\left(\mathrm{SiO}_{2}, \mathrm{PE}: \mathrm{EtOAc}=4: 1\right)$ to afford compound $14(25.7 \mathrm{mg}, 75 \%)$.

Compound 14: colorless oil; $R_{f}=0.45$ (silica gel, 2:1 PE:EtOAc); $[\alpha]_{\mathrm{D}}^{25}+70.5\left(c 0.74, \mathrm{CHCl}_{3}\right)$; IR $(\mathrm{KBr})$ 3386, 3023, 2960, 2850, 1709, 1466, 1261, $1092 \mathrm{~cm}^{-1} ;{ }^{1} \mathrm{H}$ NMR (400 MHz, $\left.\mathrm{CDCl}_{3}\right): \delta 2.99-2.86(\mathrm{~m}$, 1H), 2.51 (ddt, $J=11.7,4.6,2.3 \mathrm{~Hz}, 1 \mathrm{H}), 2.30(\mathrm{tdd}, J=13.9,6.6,2.5 \mathrm{~Hz}, 1 \mathrm{H}), 2.23-2.07$ (m, $2 \mathrm{H}), 2.02$ $(\mathrm{dd}, J=13.4,2.3 \mathrm{~Hz}, 1 \mathrm{H}), 1.99-1.86(\mathrm{~m}, 1 \mathrm{H}), 1.85-1.66(\mathrm{~m}, 5 \mathrm{H}), 1.59-1.45(\mathrm{~m}, 4 \mathrm{H}), 1.40(\mathrm{~s}, 3 \mathrm{H})$, $1.17(\mathrm{~s}, 3 \mathrm{H}), 1.10(\mathrm{~d}, J=13.5 \mathrm{~Hz}, 2 \mathrm{H}), 1.01(\mathrm{~d}, J=6.7 \mathrm{~Hz}, 3 \mathrm{H}) \mathrm{ppm} ;{ }^{13} \mathrm{C} \mathrm{NMR}\left(101 \mathrm{MHz}, \mathrm{CDCl}_{3}\right) \delta$ $221.5,73.5,60.3,52.0,46.7,44.8,43.7,43.4,41.1,40.1,32.8,30.9,29.1,26.2,22.7,22.2,20.6,20.3$ ppm; HRMS (ESI-TOF, $m / z$ ): calcd for $\mathrm{C}_{18} \mathrm{H}_{29} \mathrm{O}_{2}[\mathrm{M}+\mathrm{H}]^{+}$277.2162, found 277.2163. 


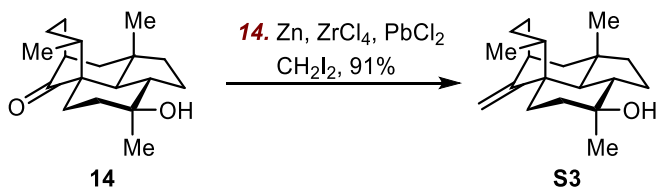

To a stirred suspension of $\mathrm{Zn}\left(643.5 \mathrm{mg}, 9.90 \mathrm{mmol}, 55.0\right.$ equiv) and $\mathrm{PbCl}_{2}$ (122.0 mg, $0.44 \mathrm{mmol}, 2.4$ equiv) in THF $(6 \mathrm{~mL})$ was added $\mathrm{CH}_{2} \mathrm{I}_{2}\left(0.27 \mathrm{~mL}, 3.29 \mathrm{mmol}, 18.3\right.$ equiv) at $0{ }^{\circ} \mathrm{C}$. The mixture was allowed to stir at this temperature for $30 \mathrm{~min}$. During this period, a suspension of $\mathrm{ZrCl}_{4}(310.0 \mathrm{mg}, 1.33$ mmol, 7.4 equiv) in THF ( $3 \mathrm{~mL}$ ) was prepared by stirring at $\mathrm{rt}$ for $20 \mathrm{~min}$. To the first mixture was added the second mixture via cannula at $0{ }^{\circ} \mathrm{C}$. The resultant mixture was allowed to stir at that temperature for $30 \mathrm{~min}$ before a solution of compound $14(50.0 \mathrm{mg}, 0.18 \mathrm{mmol}, 1.0$ equiv) in THF (2 mL) was added. The reaction mixture was allowed to stir at $0{ }^{\circ} \mathrm{C}$ for $2 \mathrm{~h}$ before it was quenched with saturated aq. $\mathrm{NaHCO}_{3}$ $(20 \mathrm{~mL})$ and extracted with EtOAc $(3 \times 25 \mathrm{~mL})$. The combined organic layers were washed with brine $(20$ $\mathrm{mL}$ ), dried over anhydrous $\mathrm{Na}_{2} \mathrm{SO}_{4}$, filtered, and concentrated in vacuo. The residue was purified by flash column chromatography $\left(\mathrm{SiO}_{2}, \mathrm{PE}: \mathrm{EA}=8: 1\right)$ to give compound $\mathbf{S 3}(45.2 \mathrm{mg}, 91 \%)$.

Compound S3: colorless oil; $R_{f}=0.82$ (silica gel, 2:1 PE:EtOAc); $[\alpha]_{\mathrm{D}}^{25}+19.6\left(c\right.$ 0.81, $\left.\mathrm{CHCl}_{3}\right)$; IR $(\mathrm{KBr})$ 3353, 3073, 2957, 1642, 1463, 1381, 1262, $1103 \mathrm{~cm}^{-1} ;{ }^{1} \mathrm{H}$ NMR $\left(400 \mathrm{MHz}, \mathrm{CDCl}_{3}\right): \delta 4.67(\mathrm{~d}, J=1.9 \mathrm{~Hz}$, $1 \mathrm{H}), 4.51(\mathrm{~d}, J=1.9 \mathrm{~Hz}, 1 \mathrm{H}), 2.64-2.54(\mathrm{~m}, 1 \mathrm{H}), 2.45-2.29(\mathrm{~m}, 1 \mathrm{H}), 2.06-1.95(\mathrm{~m}, 1 \mathrm{H}), 1.95-1.78$ (m, 5H), $1.69(\mathrm{dt}, J=12.9,3.6 \mathrm{~Hz}, 1 \mathrm{H}), 1.64-1.53(\mathrm{~m}, 4 \mathrm{H}), 1.47-1.37(\mathrm{~m}, 2 \mathrm{H}), 1.28(\mathrm{td}, J=14.2,3.6$ $\mathrm{Hz}, 1 \mathrm{H}), 1.18(\mathrm{~s}, 6 \mathrm{H}), 1.12-0.99(\mathrm{~m}, 1 \mathrm{H}), 0.99-0.91(\mathrm{~m}, 1 \mathrm{H}), 0.90(\mathrm{~d}, J=6.6 \mathrm{~Hz}, 3 \mathrm{H}) \mathrm{ppm} ;{ }^{13} \mathrm{C} \mathrm{NMR}$ $\left(101 \mathrm{MHz} \mathrm{CDCl}_{3}\right) \delta 158.7,104.0,74.1,59.9,47.8,44.8,43.8,42.1,41.6,40.2,39.6,31.0,30.8,30.2$, 29.2, 21.8, 21.5, 20.6, 20.5 ppm; HRMS (ESI-TOF, $m / z$ ): calcd for $\mathrm{C}_{19} \mathrm{H}_{29}\left[\mathrm{M}+\mathrm{H}-\mathrm{H}_{2} \mathrm{O}\right]^{+} 257.2264$, found 257.2263 .

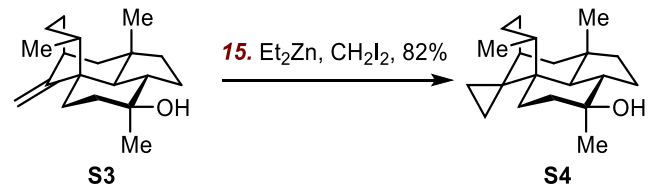

To dry $\mathrm{CH}_{2} \mathrm{Cl}_{2}(1 \mathrm{~mL})$ was added $\mathrm{Et}_{2} \mathrm{Zn}(2.0 \mathrm{M}$ in hexanes) $(0.68 \mathrm{~mL}, 1.36 \mathrm{mmol}, 8.5$ equiv) under argon. The solution was cooled in an ice bath and a solution of trifluoroacetic acid ( $61 \mu \mathrm{L}, 0.82 \mathrm{mmol}, 5.0$ equiv) in $\mathrm{CH}_{2} \mathrm{Cl}_{2}(1 \mathrm{~mL})$ was then added slowly into the reaction mixture via syringe. After stirring for $10 \mathrm{~min}$, $\mathrm{CH}_{2} \mathrm{I}_{2}(66 \mu \mathrm{L}, 0.82 \mathrm{mmol}, 5.0$ equiv) was added. After an additional $20 \mathrm{~min}$ of stirring, a solution of compound $\mathbf{S 3}$ (45.0 mg, $0.16 \mathrm{mmol}, 1.0$ equiv) in $\mathrm{CH}_{2} \mathrm{Cl}_{2}(3.3 \mathrm{~mL})$ was added, and the ice bath was removed. After stirred at $\mathrm{rt}$ for $12 \mathrm{~h}$, the reaction mixture was quenched with saturated aqueous $\mathrm{NaHCO}_{3}$ solution $(10 \mathrm{~mL})$ and extracted with EtOAc. The combined organic layers were washed with brine (10 
$\mathrm{mL}$ ), dried over $\mathrm{Na}_{2} \mathrm{SO}_{4}$, filtered and concentrated in vacuo. The crude residue was purified by column chromatography $\left(\mathrm{SiO}_{2}, \mathrm{PE}: \mathrm{EtOAc}=8: 1\right)$ to give compound $\mathbf{S 4}(39.0 \mathrm{mg}, 82 \%)$.

Compound S4: colorless oil; $R_{f}=0.82$ (silica gel, 2:1 PE:EtOAc); $[\alpha]_{\mathrm{D}}^{25}+16.5\left(c\right.$ 0.56, $\left.\mathrm{CHCl}_{3}\right)$; IR $(\mathrm{KBr})$ 3346, 2951, 2891, 1464, 1380, 1128, 1104, $1010 \mathrm{~cm}^{-1} ;{ }^{1} \mathrm{H}$ NMR (400 MHz, $\left.\mathrm{CDCl}_{3}\right): \delta 2.22(\mathrm{ddd}, J=17.9$, 13.9, 7.1 Hz, 1H), $2.02-1.84(\mathrm{~m}, 2 \mathrm{H}), 1.83-1.71(\mathrm{~m}, 2 \mathrm{H}), 1.70-1.63(\mathrm{~m}, 2 \mathrm{H}), 1.63-1.57(\mathrm{~m}, 3 \mathrm{H})$, $1.56-1.48(\mathrm{~m}, 2 \mathrm{H}), 1.44(\mathrm{dd}, J=12.6,4.0 \mathrm{~Hz}, 2 \mathrm{H}), 1.38(\mathrm{dt}, J=14.0,3.4 \mathrm{~Hz}, 1 \mathrm{H}), 1.18(\mathrm{~s}, 3 \mathrm{H}), 1.13(\mathrm{~s}$, $3 \mathrm{H}), 1.10-0.98(\mathrm{~m}, 2 \mathrm{H}), 0.94(\mathrm{~d}, J=6.7 \mathrm{~Hz}, 3 \mathrm{H}), 0.64(\mathrm{td}, J=13.9,3.7 \mathrm{~Hz}, 1 \mathrm{H}), 0.57$ (ddd, $J=9.6,5.6$, $4.1 \mathrm{~Hz}, 1 \mathrm{H}), 0.39(\mathrm{dt}, J=9.0,4.6 \mathrm{~Hz}, 1 \mathrm{H}), 0.32(\mathrm{dt}, J=9.1,4.4 \mathrm{~Hz}, 1 \mathrm{H}),-0.20(\mathrm{ddd}, J=9.2,5.9,4.7 \mathrm{~Hz}$, 1H) ppm; ${ }^{13} \mathrm{C}$ NMR (101 MHz, $\left.\mathrm{CDCl}_{3}\right) \delta$ 74.0, 57.7, 45.4, 44.8, 43.9, 41.3, 40.6, 40.4, 36.7, 30.4, 28.2, 26.9, 26.5, 25.9, 21.8, 21.6, 21.4, 20.5, 8.6, 5.6 ppm; HRMS (ESI-TOF, $m / z)$ : calcd for $\mathrm{C}_{20} \mathrm{H}_{31}[\mathrm{M}+\mathrm{H}-$ $\left.\mathrm{H}_{2} \mathrm{O}\right]^{+}$271.2420, found 271.2421.

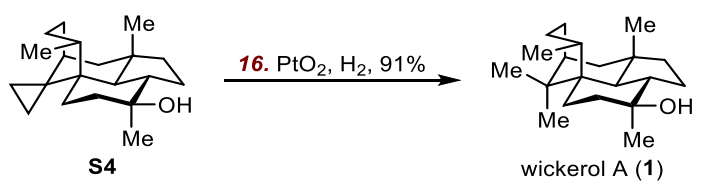

Platinum(IV) oxide (11.0 mg, 50\% w/w) was added to a solution of compound S4 (22.0 mg, $0.076 \mathrm{mmol}$, 1.0 equiv) in glacial acetic acid ( $3 \mathrm{~mL})$, and the mixture was then stirred under $\mathrm{H}_{2}(50 \mathrm{~atm})$ for $48 \mathrm{~h}$ at 60 ${ }^{\circ} \mathrm{C}$. The mixture was concentrated and purified by flash column chromatography $\left(\mathrm{SiO}_{2}, \mathrm{PE}: \mathrm{EtOAc}=10: 1\right)$ to give wickerol A (20.2 mg, 91\%).

Wickerol A (1): white solid; $R_{f}=0.80$ (silica gel, 2:1 PE:EtOAc); $[\alpha]_{\mathrm{D}}^{25}+10.5$ (c 0.10, MeOH); mp 85.0 ${ }^{\circ} \mathrm{C}$; IR (KBr) 3346, 2928, 2876, 1458, 1381, 1132, $1098 \mathrm{~cm}^{-1} ;{ }^{1} \mathrm{H}$ NMR (500 MHz, $\left.\mathrm{CDCl}_{3}\right): \delta 2.16-2.07$ (m, 2H), $2.03-1.95(\mathrm{~m}, 1 \mathrm{H}), 1.87(\mathrm{ddd}, J=13.1,10.0,6.0 \mathrm{~Hz}, 1 \mathrm{H}), 1.81-1.75(\mathrm{~m}, 1 \mathrm{H}), 1.71-1.65(\mathrm{~m}$, 2H), $1.65-1.55(\mathrm{~m}, 3 \mathrm{H}), 1.51-1.42(\mathrm{~m}, 4 \mathrm{H}), 1.40(\mathrm{~d}, J=11.2 \mathrm{~Hz}, 1 \mathrm{H}), 1.26(\mathrm{~d}, J=13.4 \mathrm{~Hz}, 1 \mathrm{H}), 1.21$ $(\mathrm{td}, J=14.0,3.5 \mathrm{~Hz}, 1 \mathrm{H}), 1.17(\mathrm{~s}, 3 \mathrm{H}), 1.05(\mathrm{~s}, 3 \mathrm{H}), 1.04(\mathrm{~s}, 3 \mathrm{H}), 1.02(\mathrm{~d}, J=7.0 \mathrm{~Hz}, 3 \mathrm{H}), 1.05-0.97$ (m, 1H), 0.94 (s, 3H) ppm; ${ }^{13} \mathrm{C}$ NMR (126 MHz, $\left.\mathrm{CDCl}_{3}\right): \delta$ 74.0, 52.0, 44.4, 43.9, 43.0, 41.0, 40.8, 39.2, 38.8, 38.7, 28.8, 26.6, 26.4, 25.7, 25.6, 24.7, 22.9, 21.6, 20.5, 19.9 ppm; HRMS (EI-TOF, $m / z)$ : calcd for $\mathrm{C}_{20} \mathrm{H}_{34} \mathrm{O}[\mathrm{M}]^{+}$290.2610, found 290.2607.

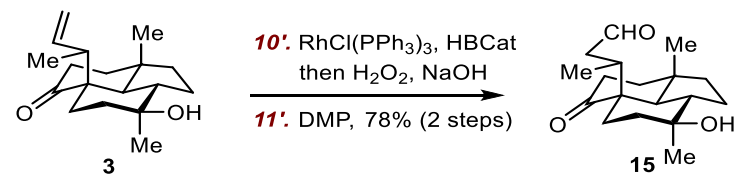

Preparation of the catalyst: Oxygen was passed through a solution of $\mathrm{Rh}\left(\mathrm{PPh}_{3}\right)_{3} \mathrm{Cl}(30.0 \mathrm{mg}, 0.032$ 
$\mathrm{mmol}, 5 \mathrm{~mol} \%$ ) in $8 \mathrm{~mL}$ of DCM for $30 \mathrm{~min}$. The solvent was removed in vacuo and the catalyst was kept under high vacuum $(<0.1$ Torr) for at least $30 \mathrm{~min}$ prior to use.

To a solution of compound 3 (180.0 mg, $0.66 \mathrm{mmol}, 1.0$ equiv) and partially oxidized $\mathrm{RhCl}\left(\mathrm{PPh}_{3}\right)_{3}$ in THF $(6 \mathrm{~mL})$ was added dropwise catecholborane $(0.36 \mathrm{~mL}, 2.38 \mathrm{mmol}, 3.6$ equiv) at $\mathrm{rt}$ under argon. After stirred at $\mathrm{rt}$ for $20 \mathrm{~h}$, the reaction mixture was cooled to $0{ }^{\circ} \mathrm{C}$ before $2.0 \mathrm{M} \mathrm{NaOH}$ solution $(14 \mathrm{~mL})$ and $30 \% \mathrm{H}_{2} \mathrm{O}_{2}(5 \mathrm{~mL})$ were added successively. The resulting mixture was warmed to room temperature and allowed to stir for $2 \mathrm{~h}$ before it was quenched with saturated aq. $\mathrm{Na}_{2} \mathrm{SO}_{3}(50 \mathrm{~mL})$. The mixture was extracted with EtOAc $(5 \times 50 \mathrm{~mL})$. The combined organic layers were washed with brine $(300 \mathrm{~mL})$, dried over anhydrous $\mathrm{MgSO}_{4}$, filtered, and concentrated in vacuo.

The crude was dissolved in $30 \mathrm{~mL}$ DCM, and DMP (560.0 mg, $1.32 \mathrm{mmol}, 2.0$ equiv) was added. The reaction was stirred at $\mathrm{rt}$ for $1.5 \mathrm{~h}$ before it was concentrated in vacuo. (Caution: The product was highly soluble in water. $)$ The crude residue was purified by flash chromatography $\left(\mathrm{SiO}_{2}, \mathrm{PE}: \mathrm{EtOAc}=2: 1\right)$ to afford compound 15 (148.5 mg, 78\%).

Compound 15: Colorless oil; $R_{f}=0.45$ (silica gel, 1:1 PE:EtOAc); $[\alpha]_{\mathrm{D}}^{25}-15.3$ (c 1.14, $\left.\mathrm{CHCl}_{3}\right)$; IR $(\mathrm{KBr})$ 3432, 2957, 2864, 1718, 1701, 1459, 1388, 1261, 1112, $1062 \mathrm{~cm}^{-1} ;{ }^{1} \mathrm{H}$ NMR (400 MHz, CDCl $): \delta 9.68$ $(\mathrm{d}, J=1.7 \mathrm{~Hz}, 1 \mathrm{H}), 3.25-3.12(\mathrm{~m}, 1 \mathrm{H}), 2.90(\mathrm{td}, J=14.2,6.4 \mathrm{~Hz}, 1 \mathrm{H}), 2.54(\mathrm{ddd}, J=13.9,10.7,5.8 \mathrm{~Hz}$ 1H), 2.39 (ddd, $J=18.2,9.5,2.0 \mathrm{~Hz}, 1 \mathrm{H}), 2.26(\mathrm{dd}, J=18.3,1.4 \mathrm{~Hz}, 1 \mathrm{H}), 2.12(\mathrm{ddd}, J=14.4,4.9,1.9$ Hz, 1H), 2.05 (ddd, $J=13.0,6.5,1.8 \mathrm{~Hz}, 1 \mathrm{H}), 2.00-1.86(\mathrm{~m}, 1 \mathrm{H}), 1.83-1.76(\mathrm{~m}, 1 \mathrm{H}), 1.74-1.66(\mathrm{~m}$, $3 \mathrm{H}), 1.66-1.52(\mathrm{~m}, 3 \mathrm{H}), 1.38(\mathrm{~s}, 3 \mathrm{H}), 1.32(\mathrm{~d}, J=7.0 \mathrm{~Hz}, 3 \mathrm{H}), 1.27(\mathrm{~d}, J=14.0 \mathrm{~Hz}, 2 \mathrm{H}), 1.15$ (s, 3H) ppm; ${ }^{13} \mathrm{C}$ NMR $\left(101 \mathrm{MHz}, \mathrm{CDCl}_{3}\right) \delta$ 216.6, 200.9, 73.8, 59.2, 55.3, 48.9, 45.6, 42.3, 41.2, 40.9, 40.4, 35.8, 30.5, 27.2, 22.9, 21.1, 20.1, 19.8 ppm; HRMS (ESI-TOF, $m / z$ ): calcd for $\mathrm{C}_{18} \mathrm{H}_{28} \mathrm{O}_{3} \mathrm{Na}[\mathrm{M}+\mathrm{Na}]^{+}$ 315.1931 , found 315.1928 .

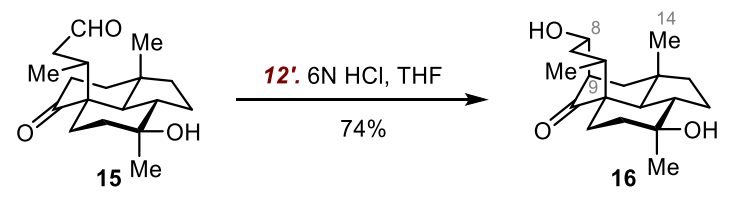

To a solution of compound 15 (27.0 mg, $0.093 \mathrm{mmol}, 1.0$ equiv) in $4 \mathrm{~mL} \mathrm{THF,} 4 \mathrm{~mL} 6 \mathrm{~N} \mathrm{HCl}$ solution was added. After $16 \mathrm{~h}$, the reaction was completed. The reaction mixture was quenched with $\mathrm{NaHCO}_{3}$ solution $(25 \mathrm{~mL})$ and extracted with EtOAc $(4 \times 10 \mathrm{~mL})$. The combined organic layers were washed with brine $(20 \mathrm{~mL})$, dried over anhydrous $\mathrm{Na}_{2} \mathrm{SO}_{4}$, and concentrated in vacuo. The crude residue was purified by flash chromatography $\left(\mathrm{SiO}_{2}, \mathrm{PE}: \mathrm{EtOAc}=1: 1\right)$ to afford compound $16(20.0 \mathrm{mg}, 74 \%)$. 
Compound 16: white solid; $R_{f}=0.17$ (silica gel, 1:1 PE:EtOAc); $[\alpha]_{\mathrm{D}}^{25}+24.9\left(c\right.$ 0.76, $\left.\mathrm{CHCl}_{3}\right) ; \operatorname{mp} 165.9$ ${ }^{\circ} \mathrm{C}$; IR (KBr) 3389, 2960, 1701, 1462, 1385, 1261, $1090 \mathrm{~cm}^{-1} ;{ }^{1} \mathrm{H}$ NMR (400 MHz, $\left.\mathrm{CDCl}_{3}\right): \delta 4.59$ (dt, $J$ $=10.9,5.6 \mathrm{~Hz}, 1 \mathrm{H}), 2.69-2.63(\mathrm{~m}, 1 \mathrm{H}), 2.42-2.35(\mathrm{~m}, 1 \mathrm{H}), 2.30(\mathrm{dt}, J=14.4,6.1 \mathrm{~Hz}, 1 \mathrm{H}), 2.21(\mathrm{dd}, J$ $=13.6,2.4 \mathrm{~Hz}, 1 \mathrm{H}), 2.10-2.03(\mathrm{~m}, 1 \mathrm{H}), 1.97-1.78(\mathrm{~m}, 3 \mathrm{H}), 1.76-1.62(\mathrm{~m}, 3 \mathrm{H}), 1.56-1.44(\mathrm{~m}, 3 \mathrm{H})$, 1.24 (s, 3H), 1.17 (s, 3H), 1.13 (d, $J=13.7 \mathrm{~Hz}, 1 \mathrm{H}), 1.06$ (d, $J=6.7 \mathrm{~Hz}, 3 \mathrm{H}), 1.04-0.89$ (m, 2H) ppm; ${ }^{13} \mathrm{C}$ NMR $\left(101 \mathrm{MHz}, \mathrm{CDCl}_{3}\right) \delta 217.5,73.4,73.3,60.1,56.5,51.0,45.0,44.5,43.0,42.5,41.0,39.9,28.8$, 25.9, 22.7, 21.5, 20.5, 20.3 ppm; HRMS (ESI-TOF, $m / z$ ): calcd for $\mathrm{C}_{18} \mathrm{H}_{28} \mathrm{O}_{3} \mathrm{Na}[\mathrm{M}+\mathrm{Na}]^{+}$315.1931, found 315.1927.

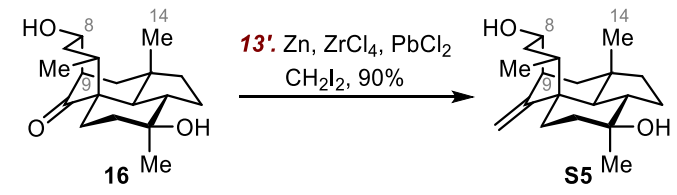

To a stirred suspension of $\mathrm{Zn}\left(620.0 \mathrm{mg}, 9.55 \mathrm{mmol}, 45.0\right.$ equiv) and $\mathrm{PbCl}_{2}$ (118.1 mg, $0.42 \mathrm{mmol}, 2.0$ equiv) in THF $(6 \mathrm{~mL})$ was added $\mathrm{CH}_{2} \mathrm{I}_{2}\left(0.26 \mathrm{~mL}, 3.15 \mathrm{mmol}, 15.0\right.$ equiv) at $0{ }^{\circ} \mathrm{C}$. The mixture was allowed to stir at this temperature for $30 \mathrm{~min}$. During this period, a suspension of $\mathrm{ZrCl}_{4}(296.0 \mathrm{mg}, 1.26$ mmol, 6.0 equiv) in THF ( $3 \mathrm{~mL}$ ) was prepared by stirring at $\mathrm{rt}$ for $20 \mathrm{~min}$. To the first mixture was added the second mixture via cannula at $0{ }^{\circ} \mathrm{C}$. The resultant mixture was allowed to stir at that temperature for $30 \mathrm{~min}$ before a solution of compound $16(60.0 \mathrm{mg}, 0.21 \mathrm{mmol}, 1.0$ equiv) in THF (3 mL) was added. The reaction mixture was allowed to stir at $0{ }^{\circ} \mathrm{C}$ for $2 \mathrm{~h}$ before it was quenched with saturated aq. $\mathrm{NaHCO}_{3}$ $(20 \mathrm{~mL})$ and extracted with EtOAc $(3 \times 50 \mathrm{~mL})$. The combined organic layers were washed with brine $(20$ $\mathrm{mL}$ ), dried over anhydrous $\mathrm{Na}_{2} \mathrm{SO}_{4}$, filtered, and concentrated in vacuo. The residue was purified by flash column chromatography $\left(\mathrm{SiO}_{2}, \mathrm{PE}: \mathrm{EA}=3: 1\right)$ to give compound $\mathbf{S 5}(53.9 \mathrm{mg}, 90 \%)$.

Compound S5: white solid; $R_{f}=0.62$ (silica gel, 1:1 PE:EtOAc); $[\alpha]_{\mathrm{D}}^{25}-3.2\left(c 0.75, \mathrm{CHCl}_{3}\right) ; \mathrm{mp} 181.6$ ${ }^{\circ} \mathrm{C}$; IR (KBr) 3355, 2959, 2933, 1640, 1332, 1380, 1261, $1102 \mathrm{~cm}^{-1}$; ${ }^{1} \mathrm{H} \mathrm{NMR}\left(400 \mathrm{MHz}, \mathrm{CDCl}_{3}\right): \delta 4.76$ $(\mathrm{d}, J=1.7 \mathrm{~Hz}, 1 \mathrm{H}), 4.63(\mathrm{~d}, J=1.7 \mathrm{~Hz}, 1 \mathrm{H}), 4.24(\mathrm{dd}, J=9.4,6.5 \mathrm{~Hz}, 1 \mathrm{H}), 2.35(\mathrm{dd}, J=5.0,2.5 \mathrm{~Hz}, 1 \mathrm{H})$, $2.23-2.09(\mathrm{~m}, 2 \mathrm{H}), 2.04(\mathrm{dd}, J=13.1,2.6 \mathrm{~Hz}, 1 \mathrm{H}), 2.00-1.92(\mathrm{~m}, 1 \mathrm{H}), 1.90(\mathrm{dt}, J=14.0,3.2 \mathrm{~Hz}, 1 \mathrm{H})$, $1.84-1.77(\mathrm{~m}, 1 \mathrm{H}), 1.72(\mathrm{dt}, J=12.8,3.6 \mathrm{~Hz}, 1 \mathrm{H}), 1.62-1.41(\mathrm{~m}, 5 \mathrm{H}), 1.30(\mathrm{td}, J=14.3,3.4 \mathrm{~Hz}, 2 \mathrm{H})$, $1.19(\mathrm{~s}, 3 \mathrm{H}), 1.02(\mathrm{~s}, 3 \mathrm{H}), 1.01-0.95(\mathrm{~m}, 1 \mathrm{H}), 0.93(\mathrm{~d}, J=6.3 \mathrm{~Hz}, 3 \mathrm{H}), 0.88-0.81(\mathrm{~m}, 2 \mathrm{H}) \mathrm{ppm} ;{ }^{13} \mathrm{C}$ NMR (101 MHz, $\left.\mathrm{CDCl}_{3}\right) \delta 154.7,106.0,73.9,73.4,59.5,52.1,45.8,44.5,43.3,43.1,41.5,41.3,39.9$, 30.4, 27.8, 21.7, 20.8, 20.52, 20.47 ppm; HRMS (ESI-TOF, $m / z$ ): calcd for $\mathrm{C}_{19} \mathrm{H}_{29} \mathrm{O}\left[\mathrm{M}+\mathrm{H}-\mathrm{H}_{2} \mathrm{O}\right]^{+}$ 273.2213, found 273.2210 . 


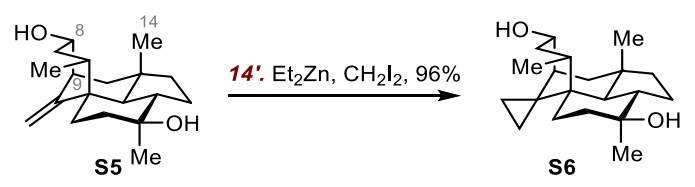

To dry $\mathrm{CH}_{2} \mathrm{Cl}_{2}(10 \mathrm{~mL})$ was added $\mathrm{Et}_{2} \mathrm{Zn}(2.0 \mathrm{M}$ in hexanes) $(1.20 \mathrm{~mL}, 2.38 \mathrm{mmol}, 7.0$ equiv) under argon. The solution was cooled in an ice bath and a solution of trifluoroacetic acid $(0.13 \mathrm{~mL}, 1.73 \mathrm{mmol}, 5.0$ equiv) in $\mathrm{CH}_{2} \mathrm{Cl}_{2}(5 \mathrm{~mL})$ was then added slowly into the reaction mixture via syringe. After stirring for $10 \mathrm{~min}, \mathrm{CH}_{2} \mathrm{I}_{2}(0.14 \mathrm{~mL}, 1.73 \mathrm{mmol}, 5.0$ equiv) was added. After an additional $20 \mathrm{~min}$ of stirring, a solution of compound $\mathbf{S 5}$ (100.0 $\mathrm{mg}, 0.34 \mathrm{mmol}, 1.0$ equiv) in $\mathrm{CH}_{2} \mathrm{Cl}_{2}(5 \mathrm{~mL})$ was added, and the ice bath was removed. After stirred at $\mathrm{rt}$ for $3 \mathrm{~h}$, the reaction mixture was quenched with saturated aqueous $\mathrm{NaHCO}_{3}$ solution $(10 \mathrm{~mL})$ and extracted with EtOAc $(3 \times 15 \mathrm{~mL})$. The combined organic layers were washed with brine $(10 \mathrm{~mL})$, dried over $\mathrm{Na}_{2} \mathrm{SO}_{4}$, filtered and concentrated in vacuo. The crude product was purified by column chromatography $\left(\mathrm{SiO}_{2}, \mathrm{PE}: \mathrm{EtOAc}=3: 1\right)$ to give compound $\mathbf{S 6}(100.6 \mathrm{mg}, 96 \%)$.

Compound S6: white solid; $R_{f}=0.38$ (silica gel, 2:1 PE:EtOAc); $[\alpha]_{\mathrm{D}}^{25}+6.5$ (c 1.00, $\left.\mathrm{CHCl}_{3}\right) ; \mathrm{mp} 201.4$ ${ }^{\circ} \mathrm{C}$; IR (KBr) 3352, 2959, 2924, 1461, 1380, 1261, 1102, $1014 \mathrm{~cm}^{-1} ;{ }^{1} \mathrm{H}$ NMR (400 MHz, $\left.\mathrm{CDCl}_{3}\right) \delta 4.24$ $(\mathrm{dd}, J=9.5,7.4 \mathrm{~Hz}, 1 \mathrm{H}), 2.18(\mathrm{dt}, J=13.5,6.8 \mathrm{~Hz}, 1 \mathrm{H}), 2.13-2.04(\mathrm{~m}, 1 \mathrm{H}), 1.88-1.71(\mathrm{~m}, 3 \mathrm{H}), 1.59$ $(\mathrm{dt}, J=13.0,3.7 \mathrm{~Hz}, 1 \mathrm{H}), 1.57-1.42(\mathrm{~m}, 6 \mathrm{H}), 1.41-1.35(\mathrm{~m}, 1 \mathrm{H}), 1.17(\mathrm{~s}, 3 \mathrm{H}), 1.08-1.00(\mathrm{~m}, 2 \mathrm{H})$, $0.99(\mathrm{~d}, J=6.6 \mathrm{~Hz}, 3 \mathrm{H}), 0.96(\mathrm{~s}, 3 \mathrm{H}), 0.64(\mathrm{td}, J=14.3,4.0 \mathrm{~Hz}, 1 \mathrm{H}), 0.63-0.59(\mathrm{~m}, 1 \mathrm{H}), 0.55(\mathrm{dt}, J=$ 9.3, 4.7 Hz, 1H), 0.37 (dt, $J=9.5,4.9 \mathrm{~Hz}, 1 \mathrm{H}),-0.17(\mathrm{ddd}, J=9.2,5.9,5.1 \mathrm{~Hz}, 1 \mathrm{H}) \mathrm{ppm} ;{ }^{13} \mathrm{C} \mathrm{NMR}(101$ $\left.\mathrm{MHz}, \mathrm{CDCl}_{3}\right) \delta$ 73.8, 72.1, 57.4, 52.3, 44.6, 43.6, 43.4, 42.3, 41.2, 40.4, 36.3, 27.4, 26.4, 25.7, 21.5, 21.3, 20.7, 20.4, 8.1, 4.3 ppm; HRMS (ESI-TOF, $m / z$ ): calcd for $\mathrm{C}_{20} \mathrm{H}_{32} \mathrm{O}_{2} \mathrm{Na}[\mathrm{M}+\mathrm{Na}]^{+} 327.2295$, found 327.2289 .

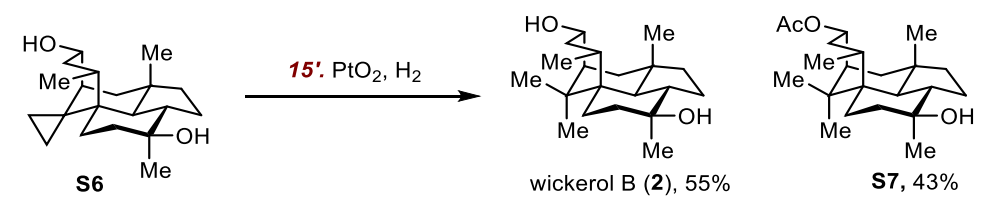

Platinum(IV) oxide (10.0 mg, 50\% w/w) was added to a solution of compound S6 $(20.0 \mathrm{mg}, 0.066 \mathrm{mmol}$, 1.0 equiv) in glacial acetic acid $(2 \mathrm{~mL})$, and the mixture was then stirred under $\mathrm{H}_{2}(50 \mathrm{~atm})$ for $48 \mathrm{~h}$ at 60 ${ }^{\circ} \mathrm{C}$. The mixture was concentrated and purified by flash column chromatography $\left(\mathrm{SiO}_{2}, \mathrm{PE}: \mathrm{EtOAc}=\right.$ $10: 1 \rightarrow 5: 1 \rightarrow 3: 1)$ to give wickerol B $(11.1 \mathrm{mg}, 55 \%)$ and $\mathbf{S 7}(9.8 \mathrm{mg}, 43 \%)$.

Wickerol B (2): white solid; $R_{f}=0.50$ (silica gel, 2:1 PE:EtOAc); $[\alpha]_{\mathrm{D}}^{25}+24.0$ (c 0.1, MeOH); mp 200.0

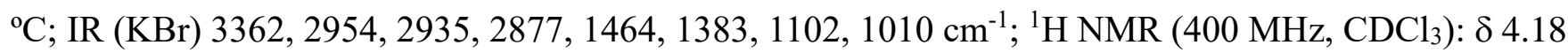


(dd, $J=10.0,6.2 \mathrm{~Hz}, 1 \mathrm{H}), 2.61(\mathrm{dt}, J=16.1,9.9 \mathrm{~Hz}, 1 \mathrm{H}), 2.06-1.97(\mathrm{~m}, 1 \mathrm{H}), 1.86-1.65(\mathrm{~m}, 4 \mathrm{H}), 1.64$ - $1.53(\mathrm{~m}, 3 \mathrm{H}), 1.50-1.38(\mathrm{~m}, 4 \mathrm{H}), 1.27$ (d, $J=12.6 \mathrm{~Hz}, 1 \mathrm{H}), 1.23(\mathrm{td}, \mathrm{J}=14.2,3.4 \mathrm{~Hz}, 1 \mathrm{H}), 1.18(\mathrm{~s}$, $3 \mathrm{H}), 1.13(\mathrm{~s}, 3 \mathrm{H}), 1.07(\mathrm{~d}, J=7.0 \mathrm{~Hz}, 3 \mathrm{H}), 1.04-0.98(\mathrm{~m}, 1 \mathrm{H}), 0.98(\mathrm{~s}, 3 \mathrm{H}), 0.91(\mathrm{~d}, J=0.7 \mathrm{~Hz}, 3 \mathrm{H})$ ppm; ${ }^{13} \mathrm{C}$ NMR (101 MHz, $\left.\mathrm{CDCl}_{3}\right): \delta$ 74.2, 73.1, 52.9, 52.3, 44.6, 43.9, 42.2, 41.5, 41.3, 39.7, 39.6, 39.3, 26.9, 26.5, 26.4, 25.4, 22.8, 21.9, 20.9, 20.1 ppm; HRMS (ESI-TOF, $m / z$ ): calcd for $\mathrm{C}_{20} \mathrm{H}_{38} \mathrm{O}_{2} \mathrm{~N}\left[\mathrm{M}+\mathrm{NH}_{4}\right]^{+}$ 324.2897, found 324.2897.

Compound S7: colorless oil; $R_{f} 0.75$ (silica gel, 2:1 PE:EtOAc); $[\alpha]_{\mathrm{D}}^{25}+17.4\left(c 0.83, \mathrm{CHCl}_{3}\right)$; IR $(\mathrm{KBr})$ 3996, 2959, 2919, 2875, 1731, 1464, 1384, 1366, 1260, 1103, $1018 \mathrm{~cm}^{-1} ;{ }^{1} \mathrm{H} \mathrm{NMR}\left(400 \mathrm{MHz}, \mathrm{CDCl}_{3}\right): \delta$ $5.12(\mathrm{dd}, J=10.3,6.2 \mathrm{~Hz}, 1 \mathrm{H}), 2.60(\mathrm{dt}, J=16.3,10.1 \mathrm{~Hz}, 1 \mathrm{H}), 2.07-2.02(\mathrm{~m}, 1 \mathrm{H}), 2.01(\mathrm{~s}, 3 \mathrm{H}), 1.86-$ $1.67(\mathrm{~m}, 5 \mathrm{H}), 1.65-1.52(\mathrm{~m}, 3 \mathrm{H}), 1.50-1.39(\mathrm{~m}, 4 \mathrm{H}), 1.29(\mathrm{~d}, J=13.0 \mathrm{~Hz}, 1 \mathrm{H}), 1.22(\mathrm{td}, J=14.0,3.4$ $\mathrm{Hz}, 1 \mathrm{H}), 1.17$ (s, 3H), $1.08(\mathrm{~s}, 3 \mathrm{H}), 1.07$ (d, J=7.8 Hz, 3H), $1.05-0.97$ (m, 1H), 0.96 (d, J = 0.8 Hz, 3H), 0.96 (s, 3H) ppm; ${ }^{13} \mathrm{C}$ NMR (101 MHz, $\left.\mathrm{CDCl}_{3}\right) \delta 170.9,76.1,73.9,51.9,48.8,44.3,43.6,41.0,40.6$, 39.3, 39.0, 38.9, 37.8, 26.3, 26.2, 25.7, 24.9, 22.5, 21.8, 21.6, 20.6, 19.8 ppm; HRMS (ESI-TOF, $m / z)$ : calcd for $\mathrm{C}_{22} \mathrm{H}_{40} \mathrm{O}_{3} \mathrm{~N}\left[\mathrm{M}+\mathrm{NH}_{4}\right]^{+} 366.3003$, found 366.3002

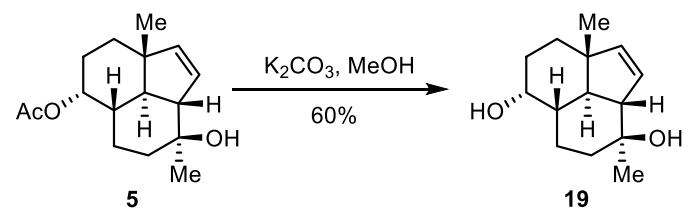

To a solution of compound 5 (12.0 mg, $0.045 \mathrm{mmol}, 1.0$ equiv) in $1 \mathrm{~mL} \mathrm{MeOH}$ was added $\mathrm{K}_{2} \mathrm{CO}_{3}(13.0$ $\mathrm{mg}, 0.09 \mathrm{mmol}, 2.0$ equiv). The reaction mixture was refluxed for $5 \mathrm{~h}$, quenched with water and extracted with EtOAc $(3 \times 5 \mathrm{~mL})$. The combined organic layers were washed with brine, dried over $\mathrm{Na}_{2} \mathrm{SO}_{4}$, and concentrated in vacuo. The crude residue was purified by flash chromatography $\left(\mathrm{SiO}_{2}, \mathrm{PE}: \mathrm{EtOAc}=1: 1\right)$ to afford compound $19(6.1 \mathrm{mg}, 60 \%)$.

Compound 19: Colorless crystal; $R_{f}=0.61$ (silica gel, 1:1 PE:EtOAc); $[\alpha]_{\mathrm{D}}^{22}=+39.4(c 0.32, \mathrm{MeOH}) ; \mathrm{mp}$ $164.3{ }^{\circ} \mathrm{C}$; IR (KBr) 3385, 2932, 2889, 1457, $1115 \mathrm{~cm}^{-1} ;{ }^{1} \mathrm{H}$ NMR $\left(400 \mathrm{MHz}, \mathrm{CDCl}_{3}\right) \delta 6.00(\mathrm{dd}, J=5.8$, $2.4 \mathrm{~Hz}, 1 \mathrm{H}), 5.94(\mathrm{dd}, J=5.8,1.2 \mathrm{~Hz}, 1 \mathrm{H}), 3.88$ (q, $J=2.7 \mathrm{~Hz}, 1 \mathrm{H}), 2.21(\mathrm{~d}, J=12.2 \mathrm{~Hz}, 1 \mathrm{H}), 1.90-$ $1.79(\mathrm{~m}, 1 \mathrm{H}), 1.77-1.70(\mathrm{~m}, 2 \mathrm{H}), 1.65(\mathrm{td}, J=13.0,4.0 \mathrm{~Hz}, 1 \mathrm{H}), 1.62-1.49(\mathrm{~m}, 4 \mathrm{H}), 1.48-1.36(\mathrm{~m}$, 2H), 1.17 (s, 3H), 0.85 (s, 3H) ppm; $\left.{ }^{13} \mathrm{C} \mathrm{NMR} \mathrm{(101} \mathrm{MHz,} \mathrm{CDCl}_{3}\right) \delta$ 145.3, 130.7, 72.9, 67.9, 54.6, 49.3, 44.5, 41.5, 36.5, 30.6, 30.4, 24.9, 21.1, 15.0 ppm; HRMS (EI-TOF, m/z): calcd for $\mathrm{C}_{14} \mathrm{H}_{22} \mathrm{O}_{2}\left[\mathrm{M}^{+}\right.$ 222.1620 , found 222.1626 . 


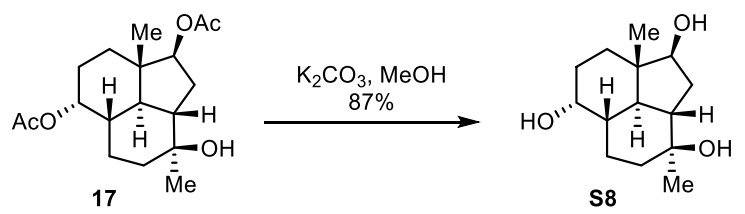

To a solution of compound 17 (2.4 g, $7.4 \mathrm{mmol}, 1.0$ equiv) in $37 \mathrm{~mL} \mathrm{MeOH}$ was added $\mathrm{K}_{2} \mathrm{CO}_{3}(1.6 \mathrm{~g}$, $29.6 \mathrm{mmol}, 4.0$ equiv). The reaction mixture was refluxed for $10 \mathrm{~h}$ before concentration in vacuo. The crude residue was purified by flash chromatography $\left(\mathrm{SiO}_{2}, \mathrm{PE}: \mathrm{EtOAc}=1: 1 \rightarrow 0: 1\right)$ to afford compound S8 (1.5 g, 87\%).

Compound S8: white solid; $R_{f}=0.15$ (silica gel, 0:1 PE:EtOAc); $[\alpha]_{\mathrm{D}}^{30}+13.9(c 2.58, \mathrm{MeOH}) ; \mathrm{mp} 117.5$ ${ }^{\circ} \mathrm{C}$; IR (KBr) 3354, 2964, 2921, 2865, 1408, 186, 1324, 1226, 1147, $1052 \mathrm{~cm}^{-1}$; ${ }^{1} \mathrm{H}$ NMR (400 MHz, MeOH- $\left.d_{4}\right) \delta 3.75(\mathrm{q}, J=2.8 \mathrm{~Hz}, 1 \mathrm{H}), 3.66(\mathrm{dd}, J=9.6,6.4 \mathrm{~Hz}, 1 \mathrm{H}), 2.00(\mathrm{ddd}, J=13.9,9.7,7.8 \mathrm{~Hz}, 1 \mathrm{H})$, $1.81-1.73(\mathrm{~m}, 1 \mathrm{H}), 1.73-1.65(\mathrm{~m}, 3 \mathrm{H}), 1.56(\mathrm{dt}, J=12.6,3.4 \mathrm{~Hz}, 1 \mathrm{H}), 1.52-1.35(\mathrm{~m}, 6 \mathrm{H}), 1.13(\mathrm{~s}$, $3 \mathrm{H}), 1.10(\mathrm{t}, J=13.2 \mathrm{~Hz}, 1 \mathrm{H}), 0.81$ (s, $3 \mathrm{H}) \mathrm{ppm} ;{ }^{13} \mathrm{C} \mathrm{NMR}\left(101 \mathrm{MHz}, \mathrm{MeOH}-d_{4}\right) \delta 82.4,73.7,68.7,48.4$, 45.2, 43.6, 42.8, 40.1, 33.0, 32.4, 30.9, 26.5, 19.7, 11.9 ppm; HRMS (ESI-TOF, $m / z$ ): calcd for $\mathrm{C}_{14} \mathrm{H}_{28} \mathrm{O}_{3} \mathrm{~N}$ $\left[\mathrm{M}+\mathrm{NH}_{4}\right]^{+}$258.2064, found 258.2063.

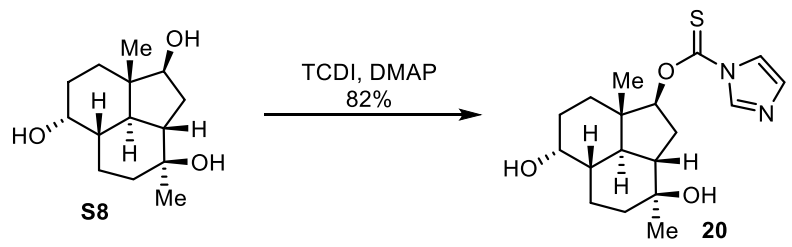

A solution of compound $\mathbf{S 8}$ (100.0 mg, $0.42 \mathrm{mmol}, 1.0$ equiv), 1,1'-thiocarbonyldiimidazole (95\%, 157.0 $\mathrm{mg}, 0.84 \mathrm{mmol}, 2.0$ equiv), and DMAP (10.0 mg, $0.084 \mathrm{mmol}, 0.2$ equiv) in acetonitrile ( $4.2 \mathrm{~mL}$ ) was stirred at room temperature for $24 \mathrm{~h}$, and then the solvent was removed under reduced pressure to give an orange oil. The crude was purified by flash chromatography $\left(\mathrm{SiO}_{2}, \mathrm{PE}:\right.$ Acetone $\left.=3: 1\right)$ to give the desired product 20 (120.0 $\mathrm{mg}, 82 \%)$.

Compound 20: white solid; $R_{f}=0.36$ (silica gel, 0:1 PE:EtOAc); $[\alpha]_{\mathrm{D}}^{25}+47.7\left(c 0.90, \mathrm{CHCl}_{3}\right) ; \mathrm{mp} 80.0$ ${ }^{\circ} \mathrm{C}$; IR (KBr) 3384, 2973, 2929, 2897, 1470, 1380, 1335, 1286, 1254, $1098 \mathrm{~cm}^{-1}$; ${ }^{1} \mathrm{H}$ NMR (400 MHz, $\left.\mathrm{CDCl}_{3}\right) \delta 8.31(\mathrm{t}, J=1.1 \mathrm{~Hz}, 1 \mathrm{H}), 7.60(\mathrm{t}, J=1.3 \mathrm{~Hz}, 1 \mathrm{H}), 7.02(\mathrm{dd}, J=1.5,0.7 \mathrm{~Hz}, 1 \mathrm{H}), 5.35(\mathrm{dd}, J=$ 9.7, $5.6 \mathrm{~Hz}, 1 \mathrm{H}), 3.88$ (s, 1H), 2.41 (ddd, $J=14.5,9.5,8.6 \mathrm{~Hz}, 1 \mathrm{H}), 1.90$ (ddd, $J=13.5,9.8,8.3 \mathrm{~Hz}, 1 \mathrm{H}$ ), $1.80-1.73(\mathrm{~m}, 2 \mathrm{H}), 1.73-1.65(\mathrm{~m}, 3 \mathrm{H}), 1.65-1.54(\mathrm{~m}, 3 \mathrm{H}), 1.54-1.43(\mathrm{~m}, 3 \mathrm{H}), 1.22(\mathrm{~s}, 3 \mathrm{H}), 1.02(\mathrm{~s}$, 3H) ppm; ${ }^{13} \mathrm{C}$ NMR $\left(101 \mathrm{MHz}, \mathrm{CDCl}_{3}\right) \delta 184.0,136.7,130.6,118.0,91.4,72.9,67.3,47.3,43.7,43.3$, 41.9, 38.4, 32.2, 30.1, 29.5, 25.3, 20.2, 13.1 ppm; HRMS (ESI-TOF, $m / z$ ): calcd for $\mathrm{C}_{18} \mathrm{H}_{27} \mathrm{~N}_{2} \mathrm{O}_{3} \mathrm{~S}[\mathrm{M}+\mathrm{H}]^{+}$ 351.1737 , found 351.1734 . 


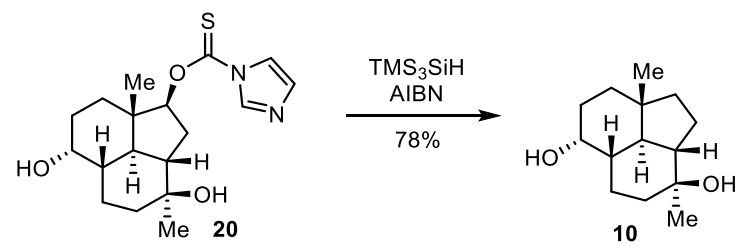

To a solution of compound 20 (300.0 mg, $0.86 \mathrm{mmol}, 1.0$ equiv) in degassed toluene ( $30 \mathrm{~mL})$ were added AIBN (36.0 mg, 0.22 mmol, 0.3 equiv) and $\mathrm{TMS}_{3} \mathrm{SiH}(0.40 \mathrm{~mL}, 1.29 \mathrm{mmol}, 1.5$ equiv). The reaction mixture was heated at $100^{\circ} \mathrm{C}$ for $2 \mathrm{~h}$, cooled to room temperature and TBAF (4.0 mL, $1 \mathrm{M}$ in THF) was then added. After stirred for $12 \mathrm{~h}$ at $\mathrm{rt}$, the reaction mixture was concentrated in vacuo. The crude residue was purified by flash chromatography $\left(\mathrm{SiO}_{2}, \mathrm{PE}: \mathrm{EtOAc}=1: 1\right)$ to afford compound $\mathbf{1 0}(149.8 \mathrm{mg}, 78 \%)$.
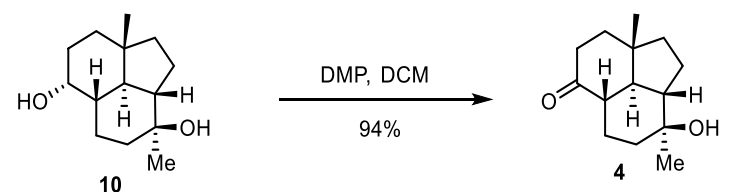

To a solution of compound 11 (1.6 g, $7.1 \mathrm{mmol}, 1.0$ equiv) in $140 \mathrm{~mL}$ DCM was added DMP (4.5 g, 10.7 mmol, 1.5 equiv). After $1 \mathrm{~h}$, the reaction mixture was quenched with saturated aqueous $\mathrm{NaHCO}_{3}$ solution $(40 \mathrm{~mL})$ and aqueous $\mathrm{Na}_{2} \mathrm{SO}_{3}$ solution $(40 \mathrm{~mL})$ and the aqueous phase was extracted with DCM $(3 \times 75$ $\mathrm{mL})$. The combined organic layers were washed with brine $(20 \mathrm{~mL})$, dried over $\mathrm{Na}_{2} \mathrm{SO}_{4}$, and concentrated in vacuo. The crude residue was purified by flash chromatography $\left(\mathrm{SiO}_{2}, \mathrm{PE}: \mathrm{EtOAc}=3: 1\right)$ to afford compound 4 (1.5 g, 94\%).

Compound 4: white solid; $R_{f}=0.61$ (silica gel, 1:1 PE:EtOAc); $[\alpha]_{\mathrm{D}}^{22}+54.5\left(c 0.81, \mathrm{CHCl}_{3}\right) ; \mathrm{mp} 136.4$ ${ }^{\circ} \mathrm{C}$; IR (KBr) 3373, 2935, 2865, 1708, 1457, 1388, 1244, $1107 \mathrm{~cm}^{-1} ;{ }^{1} \mathrm{H}$ NMR (400 MHz, $\left.\mathrm{CDCl}_{3}\right) \delta 2.47$ $(\mathrm{td}, J=14.3,13.8,6.2 \mathrm{~Hz}, 1 \mathrm{H}), 2.32(\mathrm{dd}, J=4.9,1.9 \mathrm{~Hz}, 1 \mathrm{H}), 2.18(\mathrm{ddd}, J=12.9,9.5,3.5 \mathrm{~Hz}, 1 \mathrm{H}), 2.00$ - $1.92(\mathrm{~m}, 2 \mathrm{H}), 1.92-1.87(\mathrm{~m}, 1 \mathrm{H}), 1.87-1.82(\mathrm{~m}, 1 \mathrm{H}), 1.82-1.77(\mathrm{~m}, 1 \mathrm{H}), 1.70-1.59(\mathrm{~m}, 3 \mathrm{H}), 1.58$ $(\mathrm{d}, J=4.7 \mathrm{~Hz}, 1 \mathrm{H}), 1.44-1.34(\mathrm{~m}, 2 \mathrm{H}), 1.34-1.22(\mathrm{~m}, 1 \mathrm{H}), 1.14(\mathrm{~s}, 3 \mathrm{H}), 1.10(\mathrm{~s}, 3 \mathrm{H}), 1.05(\mathrm{t}, J=13.3$ $\mathrm{Hz}, 1 \mathrm{H}) \mathrm{ppm} ;{ }^{13} \mathrm{C} \mathrm{NMR}\left(101 \mathrm{MHz}, \mathrm{CDCl}_{3}\right) \delta 212.3,73.2,56.3,49.6,48.1,41.7,39.5,39.0,38.6,37.8$, 23.6, 22.9, 20.1, 17.8 ppm; HRMS (EI-TOF, $m / z$ ): calcd for $\mathrm{C}_{14} \mathrm{H}_{22} \mathrm{O}_{2}[\mathrm{M}]^{+} 222.1620$, found 222.1624.

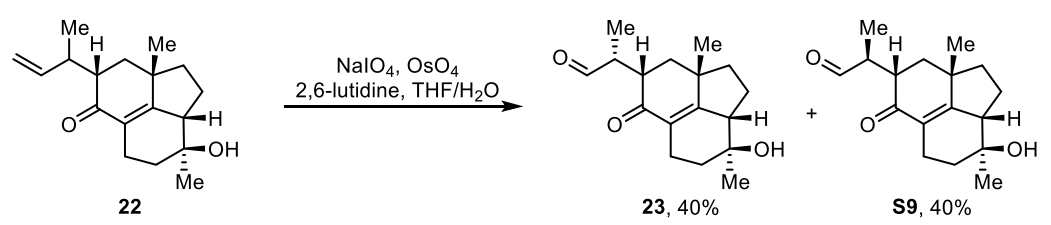

To a solution of compound 22 (5.0 mg, $0.018 \mathrm{mmol}, 1.0$ equiv) and $\mathrm{NaIO}_{4}(19.3 \mathrm{mg}, 0.09 \mathrm{mmol}, 5.0$ equiv $)$ 
in $1 \mathrm{~mL} \mathrm{THF} / \mathrm{H}_{2} \mathrm{O}(1: 1)$ was added 2,6-lutidine $\left(8.4 \mu \mathrm{L}, 0.072 \mathrm{mmol}, 4.0\right.$ equiv) and $\mathrm{OsO}_{4}\left(2 \%\right.$ in $\mathrm{H}_{2} \mathrm{O}$, $70 \mu \mathrm{L}, 5.5 \mu \mathrm{mol}, 0.3$ equiv). The reaction was stirred for $4 \mathrm{~h}$ at $\mathrm{rt}$ before it was quenched with saturated aq. $\mathrm{Na}_{2} \mathrm{SO}_{3}(10 \mathrm{~mL})$ and extracted with EtOAc $(3 \times 10 \mathrm{~mL})$. The combined organic layers were washed with brine, dried over anhydrous $\mathrm{Na}_{2} \mathrm{SO}_{4}$, and concentrated in vacuo. The crude residue was purified by PTLC (PE:EtOAc = 1:1) to afford compound 23 (2.0 mg, 40\%) and S9 (2.0 mg, 40\%).

Compound 23: white solid; $R_{f}=0.24$ (silica gel, 2:1 PE:EtOAc); $[\alpha]_{\mathrm{D}}^{25}+37.3\left(c\right.$ 0.20, $\left.\mathrm{CHCl}_{3}\right)$; IR $(\mathrm{KBr})$ 3420, 2968, 2927, 2856, 1717, 1653, 1457, 1379, 1261, $1115 \mathrm{~cm}^{-1} ;{ }^{1} \mathrm{H}$ NMR (400 MHz, CDCl $): \delta 9.75$ (s, 1H), $3.16(\mathrm{dt}, J=14.1,4.2 \mathrm{~Hz}, 1 \mathrm{H}), 2.94-2.81(\mathrm{~m}, 1 \mathrm{H}), 2.63(\mathrm{ddd}, J=7.2,7.2,3.6 \mathrm{~Hz}, 1 \mathrm{H}), 2.47$ (dd, $J=18.2,6.8 \mathrm{~Hz}, 1 \mathrm{H}), 2.21-2.00(\mathrm{~m}, 2 \mathrm{H}), 1.95(\mathrm{dd}, J=12.7,4.5 \mathrm{~Hz}, 1 \mathrm{H}), 1.89-1.73(\mathrm{~m}, 4 \mathrm{H}), 1.69-$ $1.60(\mathrm{~m}, 1 \mathrm{H}), 1.46-1.35(\mathrm{~m}, 1 \mathrm{H}), 1.27(\mathrm{~s}, 3 \mathrm{H}), 1.14(\mathrm{~d}, J=7.2 \mathrm{~Hz}, 3 \mathrm{H}), 1.03(\mathrm{~s}, 3 \mathrm{H}) \mathrm{ppm} ;{ }^{13} \mathrm{C} \mathrm{NMR}$ (101 MHz, $\left.\mathrm{CDCl}_{3}\right): \delta 204.3,198.0,169.4,126.2,71.8,49.4,46.1,45.1,42.9,41.2,40.3,37.9,22.6,22.1$, 22.0, 20.5, 9.9 ppm; HRMS (ESI-TOF, $m / z$ ): calcd for $\mathrm{C}_{17} \mathrm{H}_{25} \mathrm{O}_{3}[\mathrm{M}+\mathrm{H}]^{+}$277.1798, found 277.1797.

Compound S9: colorless oil; $R_{f}=0.25$ (silica gel, 2:1 PE:EtOAc); $[\alpha]_{\mathrm{D}}^{25}+13.8\left(\right.$ c 0.20, $\left.\mathrm{CHCl}_{3}\right)$; $\mathrm{IR}(\mathrm{KBr})$

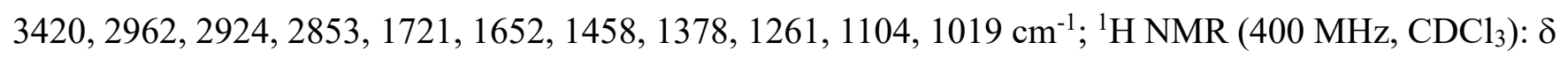
$9.79(\mathrm{~d}, J=1.1 \mathrm{~Hz}, 1 \mathrm{H}), 3.11(\mathrm{dt}, J=14.0,4.7 \mathrm{~Hz}, 1 \mathrm{H}), 3.05-2.96(\mathrm{~m}, 1 \mathrm{H}), 2.93-2.85(\mathrm{~m}, 1 \mathrm{H}), 2.52-$ $2.42(\mathrm{~m}, 1 \mathrm{H}), 2.16(\mathrm{dddd}, J=18.1,11.5,6.6,3.3 \mathrm{~Hz}, 1 \mathrm{H}), 2.10-2.00$ (m, 1H), 1.89 (dd, $J=12.7,4.3 \mathrm{~Hz}$ $1 \mathrm{H}), 1.86-1.76(\mathrm{~m}, 3 \mathrm{H}), 1.71-1.62(\mathrm{~m}, 1 \mathrm{H}), 1.61-1.57(\mathrm{~m}, 1 \mathrm{H}), 1.41-1.30(\mathrm{~m}, 1 \mathrm{H}), 1.28(\mathrm{~s}, 3 \mathrm{H})$, $1.05(\mathrm{~d}, J=7.2 \mathrm{~Hz}, 3 \mathrm{H}), 1.01$ (s, 3H) ppm; ${ }^{13} \mathrm{C} \mathrm{NMR}\left(126 \mathrm{MHz}, \mathrm{CDCl}_{3}\right) \delta 204.3,198.5,169.3,126.0$, 71.8, 49.3, 45.6, 43.0, 42.9, 41.2, 39.6, 37.9, 22.33, 22.32, 21.9, 20.4, 10.5 ppm; HRMS (ESI-TOF, $m / z)$ : calcd for $\mathrm{C}_{17} \mathrm{H}_{25} \mathrm{O}_{3}[\mathrm{M}+\mathrm{H}]^{+} 277.1798$, found 277.1797 . 


\section{NMR comparison of synthetic and natural wickerols $A$ and $B$}

\section{${ }^{1} \mathrm{H}$ NMR data comparison of synthetic and natural wickerol A}

\begin{tabular}{|c|c|c|c|c|}
\hline position & $\begin{array}{l}\text { natural wickerol } \mathrm{A}^{\mathrm{a}} \\
\left(600 \mathrm{MHz} \text { in } \mathrm{CDCl}_{3}\right)\end{array}$ & $\begin{array}{c}\text { our synthetic } \\
\text { wickerol } \mathrm{A} \\
\left(500 \mathrm{MHz} \text { in } \mathrm{CDCl}_{3}\right)\end{array}$ & $\Delta \delta$ & $\begin{array}{c}\text { Trauner's synthetic } \\
\text { wickerol } \mathrm{A}^{\mathrm{b}} \\
\left(600 \mathrm{MHz} \text { in } \mathrm{CDCl}_{3}\right)\end{array}$ \\
\hline 1 & $\begin{array}{l}1.01(\mathrm{~d}, J=11.2 \mathrm{~Hz}) \\
1.41(\mathrm{~d}, J=11.2 \mathrm{~Hz})\end{array}$ & $\begin{array}{c}1.05-0.97(\mathrm{~m}) \\
1.40(\mathrm{~d}, J=11.2 \mathrm{~Hz})\end{array}$ & $\begin{array}{c}0 \\
0.01\end{array}$ & $\begin{array}{c}1.00(\mathrm{dd}, J=10.3,1.3 \\
\mathrm{Hz}) \\
1.42-1.40(\mathrm{~m})\end{array}$ \\
\hline 2 & $\begin{array}{l}1.56(\mathrm{~m}) \\
1.78(\mathrm{~m})\end{array}$ & $\begin{array}{l}1.61-1.54(\mathrm{~m}) \\
1.81-1.75(\mathrm{~m})\end{array}$ & $\begin{array}{c}-0.01 \\
0\end{array}$ & $\begin{array}{l}1.58-1.54(\mathrm{~m}) \\
1.82-1.75(\mathrm{~m})\end{array}$ \\
\hline $2 \mathrm{a}$ & $\begin{array}{c}1.87(\mathrm{ddd}, J=13.4,10.0 \\
6.1 \mathrm{~Hz})\end{array}$ & $\begin{array}{c}1.87(\mathrm{ddd}, J=13.1, \\
10.0,6.0 \mathrm{~Hz})\end{array}$ & 0 & $\begin{array}{c}1.87(\mathrm{ddd}, J=13.2 \\
10.0,6.1 \mathrm{~Hz})\end{array}$ \\
\hline 4 & $\begin{array}{c}1.44(\mathrm{~m}) \\
1.57(\mathrm{ddd}, J=12.8,3.6 \\
3.5 \mathrm{~Hz})\end{array}$ & $\begin{array}{l}1.48-1.40(\mathrm{~m}) \\
1.61-1.55(\mathrm{~m})\end{array}$ & $\begin{array}{c}0 \\
-0.01\end{array}$ & $\begin{array}{c}1.45-1.42(\mathrm{~m}, 1 \mathrm{H}) \\
1.58(\mathrm{dt}, J=12.7,3.5 \\
\mathrm{Hz})\end{array}$ \\
\hline 5 & $\begin{array}{c}1.21(\mathrm{ddd}, J=14.2,14.2 \\
3.5 \mathrm{~Hz}) \\
1.68(\mathrm{~m})\end{array}$ & $\begin{array}{c}1.21(\mathrm{td}, J=14.0,3.5 \\
\mathrm{Hz}) \\
1.71-1.65(\mathrm{~m})\end{array}$ & $\begin{array}{l}0 \\
0\end{array}$ & $\begin{array}{c}1.21(\mathrm{td}, \mathrm{J}=14.2,3.5 \\
\mathrm{Hz}) \\
1.71-1.68(\mathrm{~m})\end{array}$ \\
\hline 6 & $2.11(\mathrm{~m})$ & $2.16-2.07(\mathrm{~m})$ & 0 & $2.14-2.10(\mathrm{~m})$ \\
\hline 7 & $\begin{array}{c}1.46(\mathrm{~m}) \\
2.00(\mathrm{dddd}, J=15.5, \\
11.2,11.2,2.6 \mathrm{~Hz})\end{array}$ & $\begin{array}{l}1.50-1.42(\mathrm{~m}) \\
2.03-1.95(\mathrm{~m})\end{array}$ & $\begin{array}{c}0 \\
0.01\end{array}$ & $\begin{array}{c}1.48-1.45(\mathrm{~m}) \\
2.00(\mathrm{dtd}, J=15.6 \\
11.0,2.7 \mathrm{~Hz})\end{array}$ \\
\hline 8 & $\begin{array}{l}1.61(\mathrm{~m}) \\
2.09(\mathrm{~m})\end{array}$ & $\begin{array}{l}1.65-1.57(\mathrm{~m}) \\
2.12-2.07(\mathrm{~m})\end{array}$ & $\begin{array}{l}0 \\
0\end{array}$ & $\begin{array}{c}1.64-1.60(\mathrm{~m}, 1 \mathrm{H}) \\
2.10(\mathrm{~s})\end{array}$ \\
\hline 9 & $1.48(\mathrm{~m})$ & $1.51-1.46(\mathrm{~m})$ & 0 & $1.51-1.48(\mathrm{~m})$ \\
\hline 10 & $\begin{array}{c}1.49(\mathrm{~m}) \\
1.67(\mathrm{dd}, J=12.3,4.2 \\
\mathrm{Hz})\end{array}$ & $\begin{array}{l}1.52-1.46(\mathrm{~m}) \\
1.70-1.65(\mathrm{~m})\end{array}$ & $\begin{array}{c}0 \\
0.01\end{array}$ & $\begin{array}{l}1.51-1.48(\mathrm{~m}) \\
1.69-1.66(\mathrm{~m})\end{array}$ \\
\hline $10 \mathrm{~b}$ & $1.27(\mathrm{~d}, J=13.4 \mathrm{~Hz})$ & $1.26(\mathrm{~d}, J=13.4 \mathrm{~Hz})$ & 0.01 & $1.27(\mathrm{~d}, J=13.5 \mathrm{~Hz})$ \\
\hline 12 & $1.17(\mathrm{~s})$ & $1.17(\mathrm{~s})$ & 0 & $1.18(\mathrm{~s})$ \\
\hline 13 & $1.02(\mathrm{~d}, J=7.0 \mathrm{~Hz})$ & $1.02(\mathrm{~d}, J=7.0 \mathrm{~Hz})$ & 0 & $1.02(\mathrm{~d}, J=7.0 \mathrm{~Hz})$ \\
\hline 14 & $1.046(\mathrm{~s})$ & $1.044(\mathrm{~s})$ & 0.002 & $1.05(\mathrm{~s})$ \\
\hline 15 & $0.94(\mathrm{~s})$ & $0.94(\mathrm{~s})$ & 0 & $0.94(\mathrm{~s})$ \\
\hline 16 & $1.051(\mathrm{~s})$ & $1.049(\mathrm{~s})$ & 0.002 & $1.05(\mathrm{~s})$ \\
\hline
\end{tabular}

aYamamoto, T.; Izumi, N.; Ui, H.; Sueki, A.; Masuma, R.; Nonaka, K.; Hirose, T.; Sunazuka, T.; Nagai, T.;

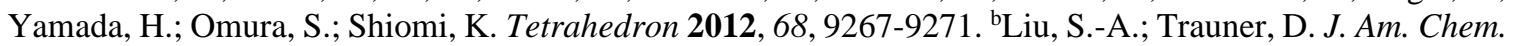
Soc. 2017, 139, 9491-9494. 


\section{${ }^{13} \mathrm{C}$ NMR data comparison of synthetic and natural wickerol $A$}

\begin{tabular}{|c|c|c|c|c|}
\hline position & $\begin{array}{l}\text { natural wickerol } \mathrm{A}^{\mathrm{a}} \\
\left(126 \mathrm{MHz} \text { in } \mathrm{CDCl}_{3}\right)\end{array}$ & $\begin{array}{l}\text { our synthetic wickerol A } \\
\qquad\left(126 \mathrm{MHz} \text { in } \mathrm{CDCl}_{3}\right)\end{array}$ & $\Delta \delta$ & $\begin{array}{c}\text { Trauner's synthetic } \\
\text { wickerol } \mathrm{A}^{\mathrm{b}} \\
\left(101 \mathrm{MHz} \text { in } \mathrm{CDCl}_{3}\right)\end{array}$ \\
\hline 1 & 43.9 & 43.9 & 0 & 43.0 \\
\hline 2 & 21.6 & 21.6 & 0 & 21.7 \\
\hline $2 \mathrm{a}$ & 44.4 & 44.4 & 0 & 44.5 \\
\hline 3 & 73.9 & 74.0 & -0.1 & 74.1 \\
\hline 4 & 40.8 & 40.8 & 0 & 40.9 \\
\hline 5 & 26.4 & 26.4 & 0 & 26.5 \\
\hline $5 a$ & 38.8 & 38.8 & 0 & 38.9 \\
\hline 6 & 26.6 & 26.6 & 0 & 26.8 \\
\hline 7 & 28.8 & 28.8 & 0 & 29.0 \\
\hline 8 & 25.7 & 25.7 & 0 & 25.8 \\
\hline 9 & 41.1 & 41.0 & 0.1 & 41.1 \\
\hline 10 & 43.0 & 43.0 & 0 & 43.1 \\
\hline $10 \mathrm{a}$ & 39.2 & 39.2 & 0 & 39.4 \\
\hline $10 \mathrm{~b}$ & 52.0 & 52.0 & 0 & 52.1 \\
\hline 11 & 38.7 & 38.7 & 0 & 38.8 \\
\hline 12 & 20.5 & 20.5 & 0 & 20.6 \\
\hline 13 & 22.9 & 22.9 & 0 & 23.1 \\
\hline 14 & 19.9 & 19.9 & 0 & 20.1 \\
\hline 15 & 25.6 & 25.6 & 0 & 25.8 \\
\hline 16 & 24.6 & 24.7 & -0.1 & 24.8 \\
\hline
\end{tabular}


NMR spectra comparison of synthetic and natural wickerol A

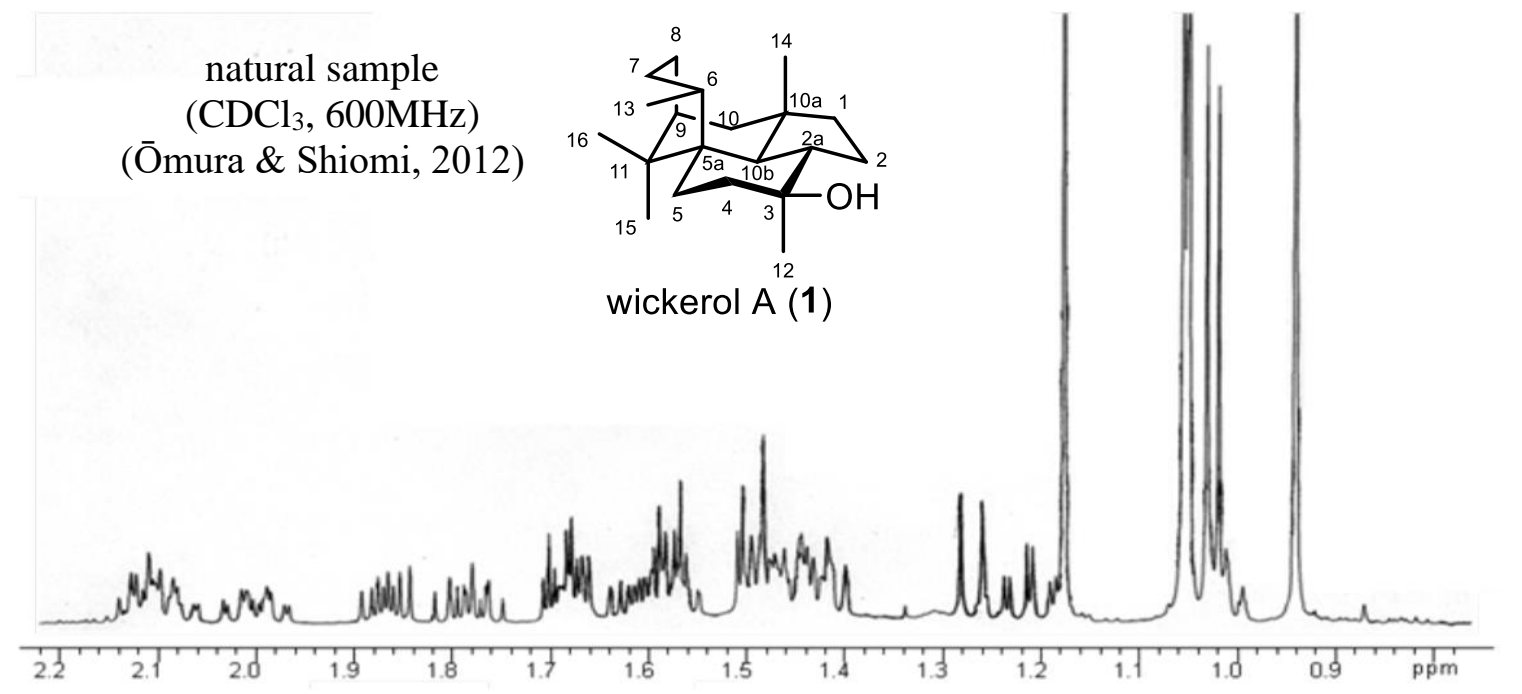

natural sample
$\left(\mathrm{CDCl}_{3}, 400 \mathrm{MHz}\right)$

(Ji, 2018)
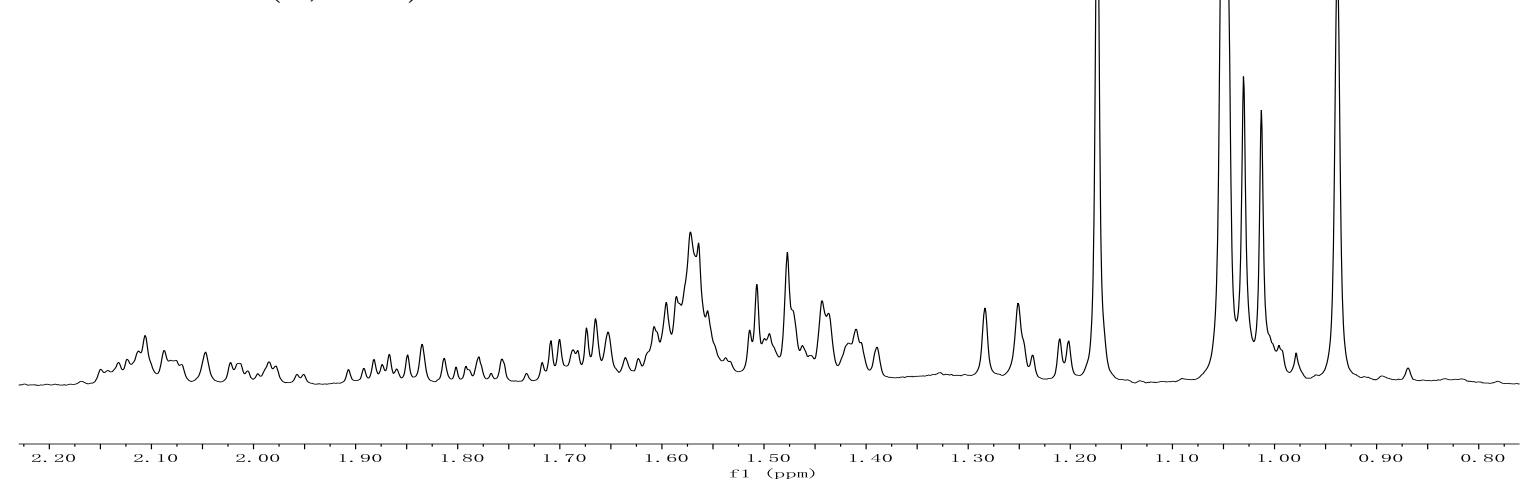

synthetic sample

$\left(\mathrm{CDCl}_{3}, 500 \mathrm{MHz}\right)$

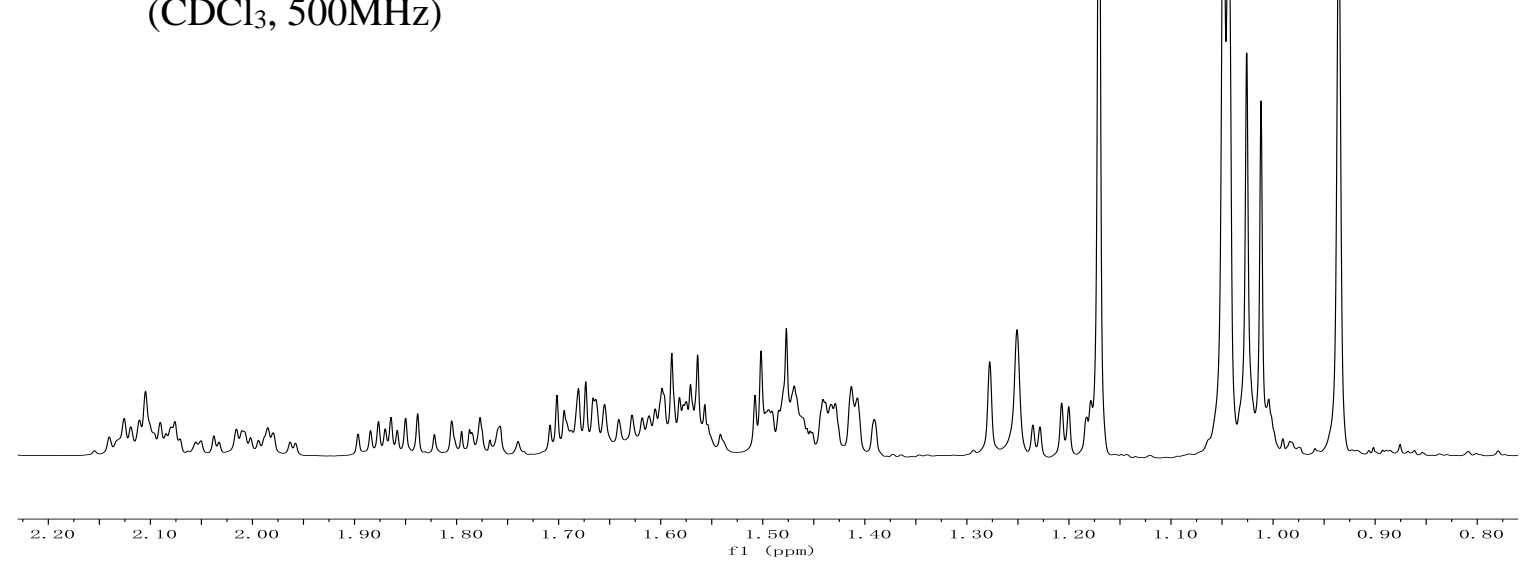




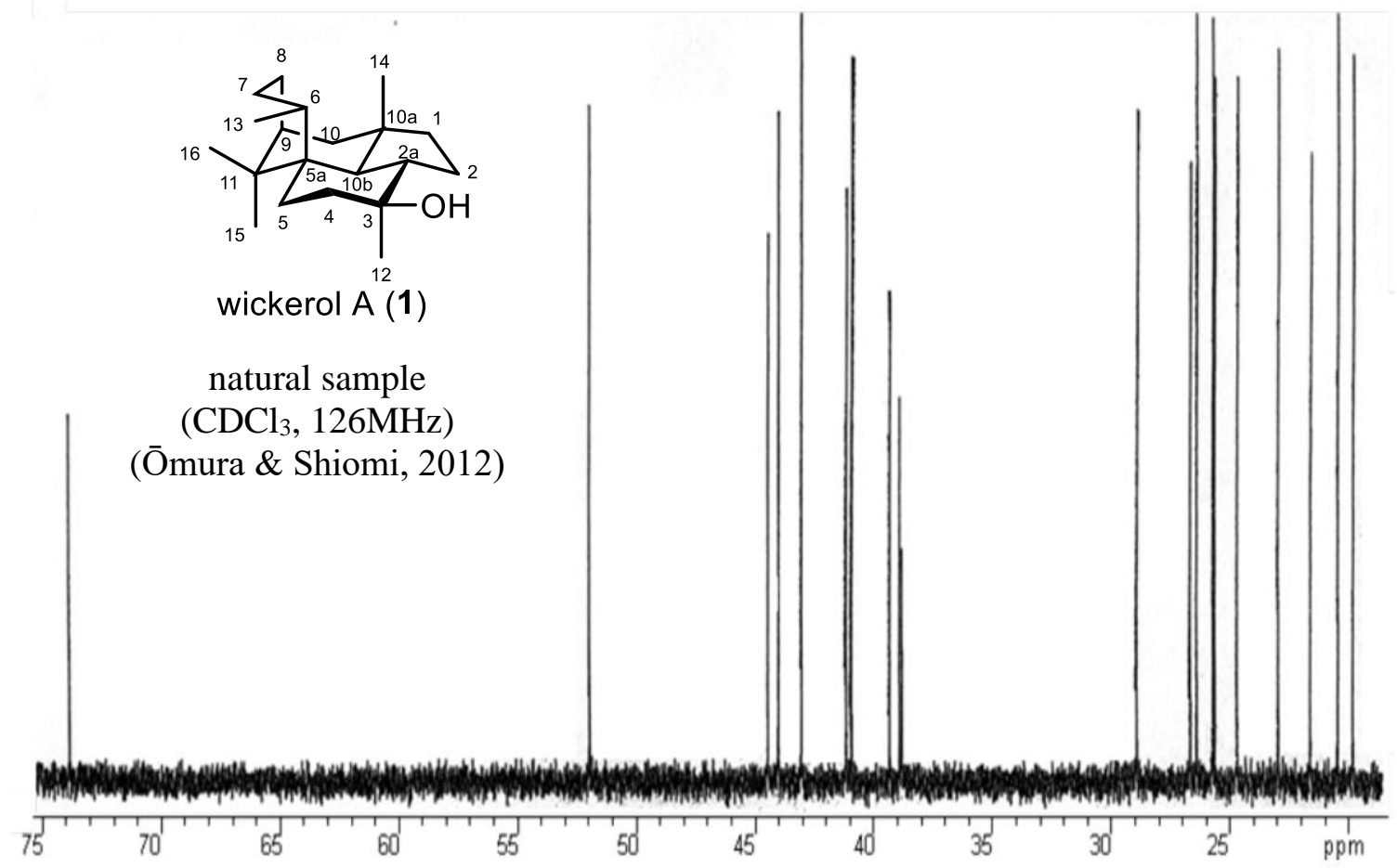

synthetic sample

$\left(\mathrm{CDCl}_{3}, 126 \mathrm{MHz}\right)$

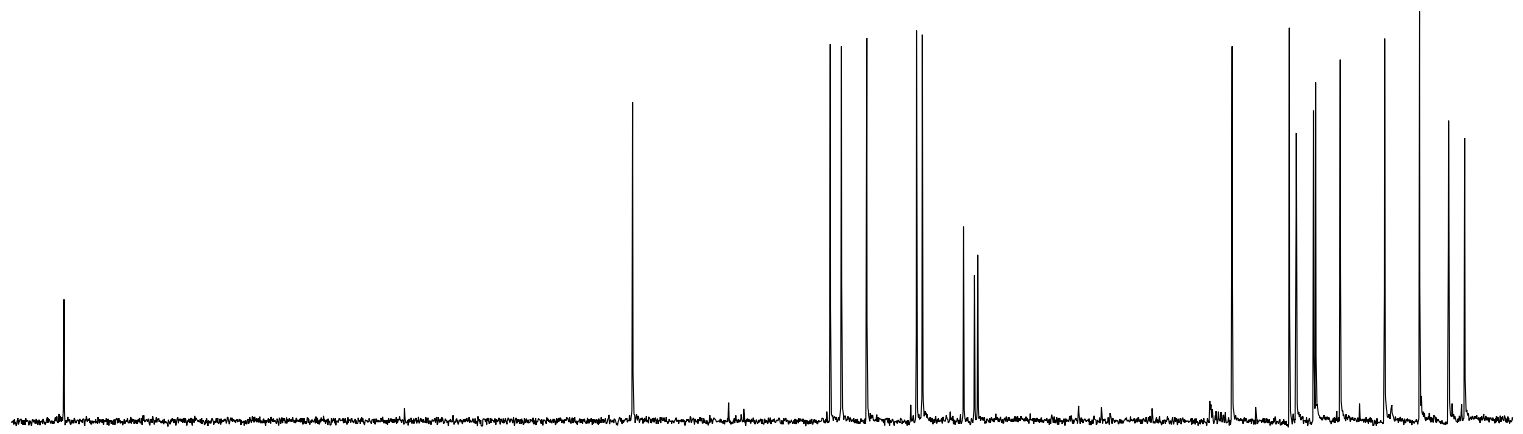




\section{${ }^{1} \mathrm{H}$ NMR data comparison of synthetic and natural wickerol B}

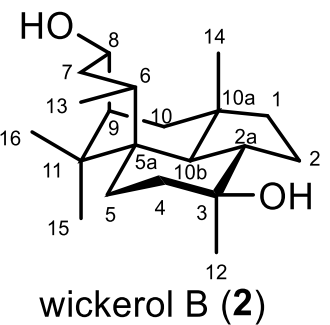

\begin{tabular}{|c|c|c|c|}
\hline position & $\begin{array}{l}\text { natural wickerol } \mathrm{B}^{\mathrm{a}} \\
\left(400 \mathrm{MHz} \text { in } \mathrm{CDCl}_{3}\right)\end{array}$ & $\begin{array}{l}\text { our synthetic wickerol B } \\
\left(400 \mathrm{MHz} \text { in } \mathrm{CDCl}_{3}\right)\end{array}$ & $\Delta \delta$ \\
\hline 1 & $\begin{array}{l}1.02(\mathrm{~m}) \\
1.43(\mathrm{~m})\end{array}$ & $\begin{array}{c}0.98-1.04(\mathrm{~m}) \\
1.38-1.45(\mathrm{~m})\end{array}$ & $\begin{array}{l}0.01 \\
0.01\end{array}$ \\
\hline 2 & $\begin{array}{l}1.58(\mathrm{~m}) \\
1.79(\mathrm{~m})\end{array}$ & $\begin{array}{l}1.53-1.61(\mathrm{~m}) \\
1.73-1.81(\mathrm{~m})\end{array}$ & $\begin{array}{l}0.01 \\
0.02\end{array}$ \\
\hline $2 \mathrm{a}$ & $1.81(\mathrm{ddd}, J=13.2,10.1,6.1 \mathrm{~Hz})$ & $1.82(\mathrm{ddd}, J=13.0,9.9,5.8 \mathrm{~Hz})$ & -0.01 \\
\hline 4 & $\begin{array}{l}1.45(\mathrm{~m}) \\
1.62(\mathrm{~m})\end{array}$ & $\begin{array}{l}1.41-1.49(\mathrm{~m}) \\
1.59-1.66(\mathrm{~m})\end{array}$ & $\begin{array}{c}0 \\
0\end{array}$ \\
\hline 5 & $\begin{array}{c}1.24(\mathrm{ddd}, J=14.1,14.1,3.3 \mathrm{~Hz}) \\
1.74(\mathrm{ddd}, J=14.1,3.6,3.3 \mathrm{~Hz})\end{array}$ & $\begin{array}{c}1.23(\mathrm{td}, J=14.2,3.4 \mathrm{~Hz}) \\
1.70-1.76(\mathrm{~m})\end{array}$ & $\begin{array}{l}0.01 \\
0.01\end{array}$ \\
\hline 6 & $2.01(\mathrm{ddq}, J=10.1,9.1,7.1 \mathrm{~Hz})$ & $1.97-2.06(\mathrm{~m})$ & 0 \\
\hline 7 & $\begin{array}{c}1.43(\mathrm{ddd}, J=16.3,9.1,6.1 \mathrm{~Hz}) \\
2.61(\mathrm{ddd}, J=16.3,10.1,9.1 \mathrm{~Hz})\end{array}$ & $\begin{array}{c}1.39-1.47(\mathrm{~m}) \\
2.61(\mathrm{dt}, J=16.1,9.9 \mathrm{~Hz})\end{array}$ & $\begin{array}{l}0 \\
0\end{array}$ \\
\hline 8 & $4.18(\mathrm{dd}, J=10.1,6.1 \mathrm{~Hz})$ & $4.18(\mathrm{dd}, J=10.0,6.2 \mathrm{~Hz})$ & 0 \\
\hline 9 & $1.42(\mathrm{~m})$ & $1.38-1.42(\mathrm{~m})$ & 0.02 \\
\hline 10 & $\begin{array}{l}1.64(\mathrm{dd}, J=3.1,14.1 \mathrm{~Hz}) \\
1.74(\mathrm{dd}, J=14.1,5.0 \mathrm{~Hz})\end{array}$ & $\begin{array}{l}1.58-1.67(\mathrm{~m}) \\
1.69-1.76(\mathrm{~m})\end{array}$ & $\begin{array}{l}0.01 \\
0.01\end{array}$ \\
\hline $10 \mathrm{~b}$ & $1.28(\mathrm{~d}, J=13.2 \mathrm{~Hz})$ & $1.27(\mathrm{~d}, J=12.6 \mathrm{~Hz})$ & 0.01 \\
\hline 12 & $1.18(\mathrm{~s})$ & $1.18(\mathrm{~s})$ & 0 \\
\hline 13 & $1.07(\mathrm{~d}, J=7.1 \mathrm{~Hz})$ & $1.07(\mathrm{~d}, J=7.0 \mathrm{~Hz})$ & 0 \\
\hline 14 & $0.91(\mathrm{~d}, J=1.1 \mathrm{~Hz})$ & $0.91(\mathrm{~d}, J=0.7 \mathrm{~Hz})$ & 0 \\
\hline 15 & $0.98(\mathrm{~s})$ & $0.98(\mathrm{~s})$ & 0 \\
\hline 16 & $1.13(\mathrm{~s})$ & $1.13(\mathrm{~s})$ & 0 \\
\hline
\end{tabular}

aYamamoto, T.; Izumi, N.; Ui, H.; Sueki, A.; Masuma, R.; Nonaka, K.; Hirose, T.; Sunazuka, T.; Nagai, T.; Yamada, H.; Omura, S.; Shiomi, K. Tetrahedron 2012, 68, 9267-9271. 


\section{${ }^{13} \mathrm{C}$ NMR data comparison of synthetic and natural wickerol $B$}

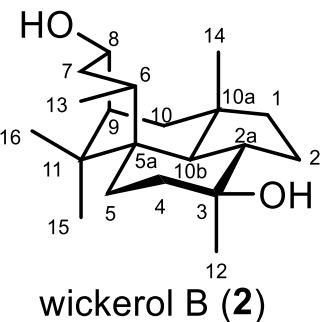

\begin{tabular}{|c|c|c|c|}
\hline position & $\begin{array}{l}\text { natural wickerol } \mathrm{B}^{\mathrm{a}} \\
\left(101 \mathrm{MHz} \text { in } \mathrm{CDCl}_{3}\right)\end{array}$ & $\begin{array}{l}\text { our synthetic wickerol B } \\
\left(101 \mathrm{MHz} \text { in } \mathrm{CDCl}_{3}\right)\end{array}$ & $\Delta \delta$ \\
\hline 1 & 43.9 & 43.9 & 0 \\
\hline 2 & 22.2 & 21.9 & 0.3 \\
\hline $2 \mathrm{a}$ & 44.6 & 44.6 & 0 \\
\hline 3 & 73.8 & 74.2 & -0.4 \\
\hline 4 & 41.4 & 41.3 & 0.1 \\
\hline 5 & 26.8 & 26.5 & 0.3 \\
\hline $5 a$ & 39.3 & 39.3 & 0 \\
\hline 6 & 27.1 & 26.9 & 0.2 \\
\hline 7 & 42.3 & 42.2 & 0.1 \\
\hline 8 & 72.7 & 73.1 & -0.4 \\
\hline 9 & 52.8 & 52.9 & -0.1 \\
\hline 10 & 41.6 & 41.5 & 0.1 \\
\hline $10 \mathrm{a}$ & 39.8 & 39.7 & 0.1 \\
\hline $10 \mathrm{~b}$ & 52.2 & 52.3 & -0.1 \\
\hline 11 & 39.6 & 39.6 & 0 \\
\hline 12 & 21.3 & 20.9 & 0.4 \\
\hline 13 & 23.1 & 22.8 & 0.3 \\
\hline 14 & 20.5 & 20.1 & 0.4 \\
\hline 15 & 26.6 & 26.4 & 0.2 \\
\hline 16 & 25.6 & 25.4 & 0.2 \\
\hline
\end{tabular}

a'Yamamoto, T.; Izumi, N.; Ui, H.; Sueki, A.; Masuma, R.; Nonaka, K.; Hirose, T.; Sunazuka, T.; Nagai, T.; Yamada, H.; Omura, S.; Shiomi, K. Tetrahedron 2012, 68, 9267-9271. 
NMR spectra comparison of synthetic and natural wickerol B

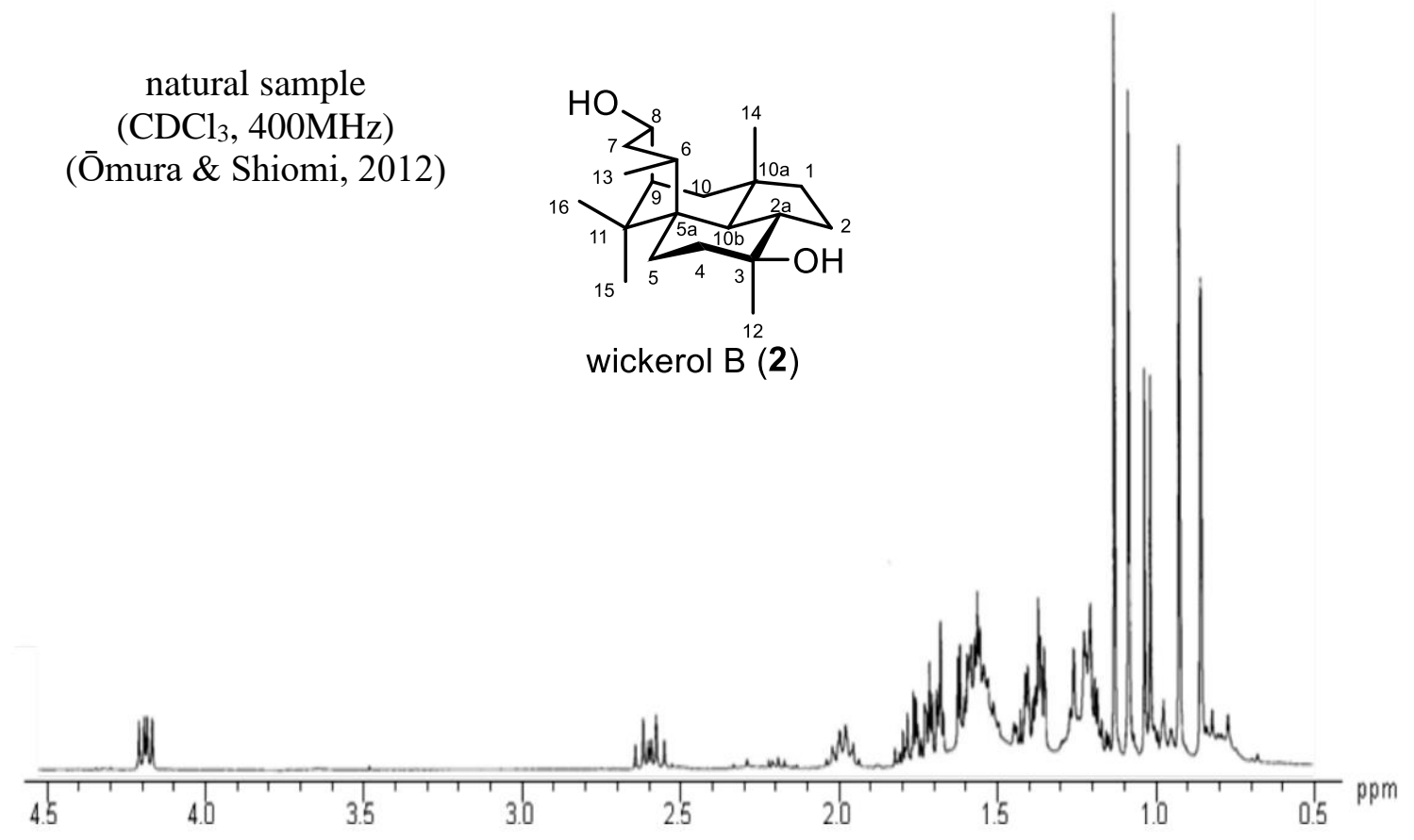

synthetic sample

$\left(\mathrm{CDCl}_{3}, 400 \mathrm{MHz}\right)$

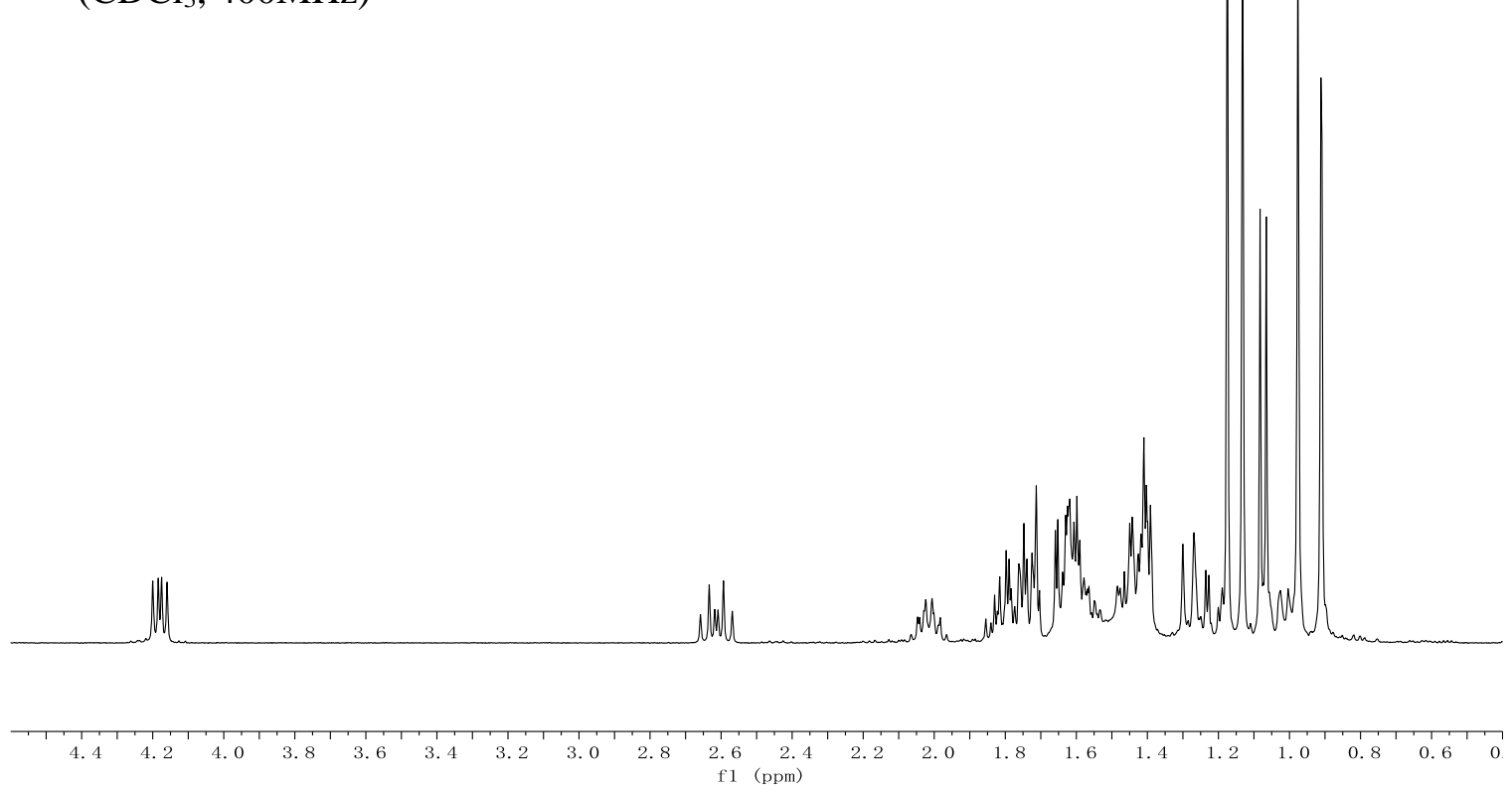




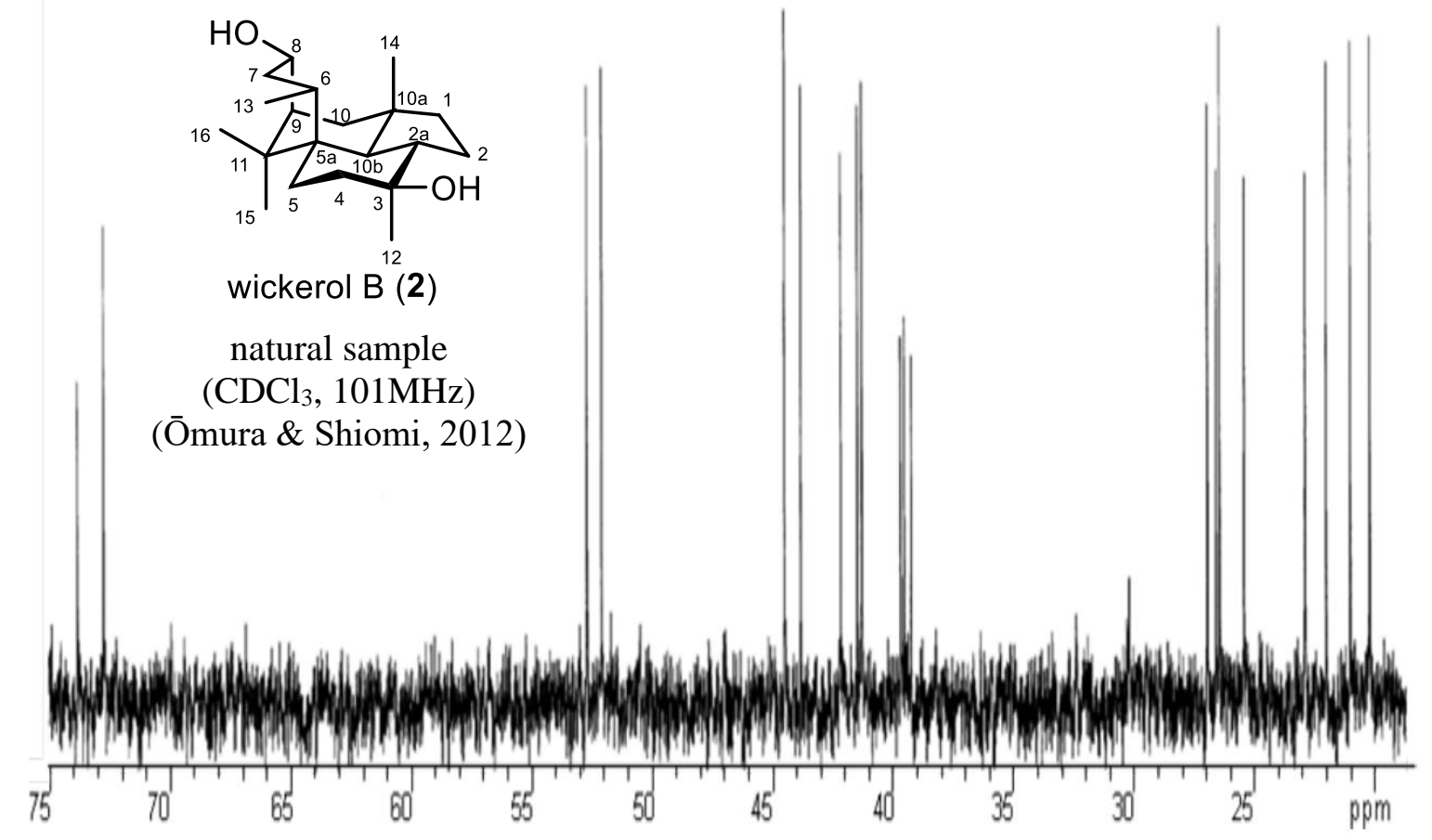

synthetic sample

$\left(\mathrm{CDCl}_{3}, 101 \mathrm{MHz}\right)$

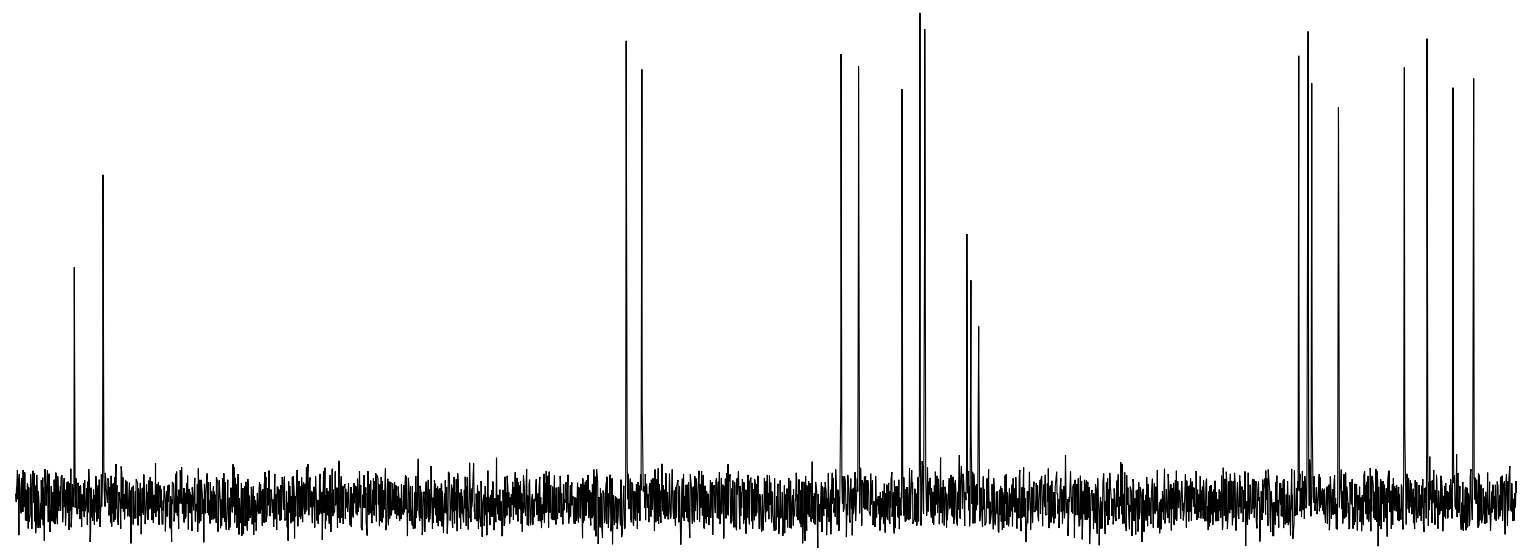

$\begin{array}{llllllllllllllllllllllllllllllllll}74 & 72 & 70 & 68 & 66 & 64 & 62 & 60 & 58 & 56 & 54 & 52 & 50 & 48 & 46 & 44 & 42 & 40 & 38 & 36 & 34 & 32 & 30 & 28 & 26 & 24 & 22 & 20 & 1\end{array}$ 


\section{Optical rotation and ECD of wickerols A and B}
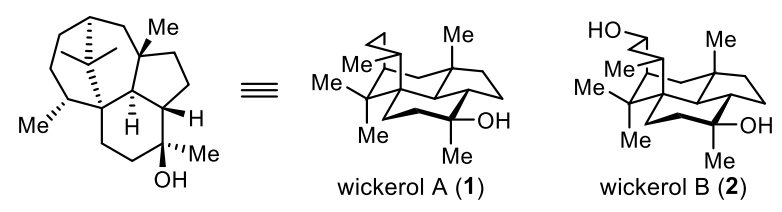

\section{Optical rotation of synthetic and natural wickerols $A$ and $B$}

\begin{tabular}{|c|c|c|c|}
\hline & wickerol A & wickerol B & $\begin{array}{l}\text { trichodermanin } \mathrm{A} \\
=\text { wickerol } \mathrm{B}\end{array}$ \\
\hline $\begin{array}{c}\text { natural sample } \\
\text { (Ōmura \& } \\
\text { Shiomi) }\end{array}$ & $\begin{array}{l}{[\alpha]_{\mathrm{D}}^{31}-2.8(\mathrm{c} 0.10, \mathrm{MeOH})^{\mathrm{a}}} \\
{[\alpha]_{\mathrm{D}}^{31}+1.6\left(\mathrm{c} 0.10, \mathrm{CHCl}_{3}\right)^{\mathrm{b}}}\end{array}$ & {$[\alpha]_{\mathrm{D}}^{31}+12.3(\mathrm{c} 0.10, \mathrm{MeOH})$} & - \\
\hline $\begin{array}{l}\text { natural sample } \\
\qquad(\mathrm{Qin})^{\mathrm{c}}\end{array}$ & - & - & $\begin{array}{c}{[\alpha]_{\mathrm{D}}^{20}+23.0(\mathrm{c} 0.04} \\
\text { acetone })\end{array}$ \\
\hline $\begin{array}{l}\text { natural sample } \\
\qquad(\mathrm{Ji})^{\mathrm{d}}\end{array}$ & $\begin{array}{l}{[\alpha]_{\mathrm{D}}^{25}+8.6(c 0.10, \mathrm{MeOH})} \\
{[\alpha]_{\mathrm{D}}^{25}+8.4\left(\mathrm{c} 0.23, \mathrm{CHCl}_{3}\right)}\end{array}$ & - & - \\
\hline $\begin{array}{l}\text { synthetic sample } \\
\text { (Trauner) }^{\mathrm{e}}\end{array}$ & {$[\alpha]_{\mathrm{D}}^{25}+21.7\left(\mathrm{c} 0.23, \mathrm{CHCl}_{3}\right)$} & - & - \\
\hline $\begin{array}{c}\text { synthetic sample } \\
\text { (this work) }\end{array}$ & $\begin{array}{c}{[\alpha]_{\mathrm{D}}^{25}+10.5(c 0.10, \mathrm{MeOH})} \\
{[\alpha]_{\mathrm{D}}^{25}+8.5\left(\mathrm{c} 0.23, \mathrm{CHCl}_{3}\right)}\end{array}$ & $\begin{array}{l}{[\alpha]_{\mathrm{D}}^{25}+24.0(c 0.10, \mathrm{MeOH})} \\
{[\alpha]_{\mathrm{D}}^{25}+21.4(\mathrm{c} 0.04, \text { acetone })}\end{array}$ & - \\
\hline \multicolumn{4}{|c|}{ 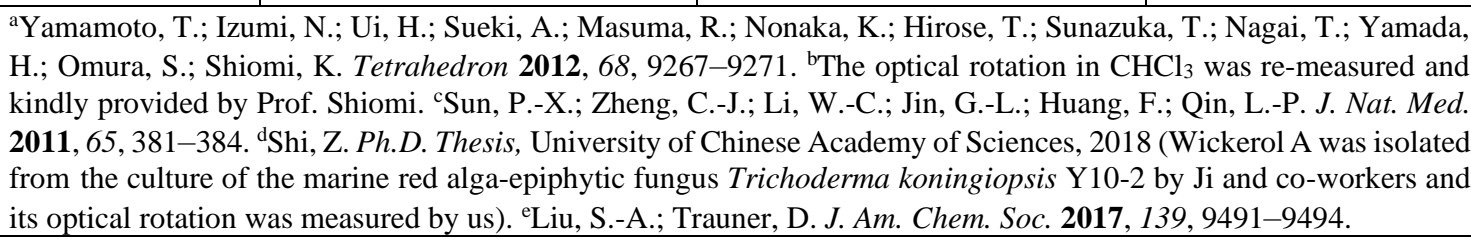 } \\
\hline
\end{tabular}

The optical rotation of our synthetic wickerol B matches well with the reported values: $[\alpha]_{\mathrm{D}}^{25}+24.1(\mathrm{c} 0.10, \mathrm{MeOH})\left(\right.$ synthetic wickerol B) $v s[\alpha]_{\mathrm{D}}^{31}+12.3(\mathrm{c} 0.10, \mathrm{MeOH})$ (natural wickerol B); ${ }^{5}[\alpha]_{\mathrm{D}}^{25}+21.4$ (c 0.04 , acetone) (synthetic wickerol B) $v s[\alpha]_{\mathrm{D}}^{20}+23.0$ (c 0.04 , acetone) (natural trichodermanin A), ${ }^{6}$ which suggests synthetic wickerol B has the same absolute configuration with the natural sample.

However, the optical rotation of our synthetic wickerol A does not match with Ōmura's originally reported value in $\mathrm{MeOH},{ }^{5}$ but is in good agreement with that of Ji's isolated sample. ${ }^{7}$ Due to the poor solubility of wickerol A in $\mathrm{MeOH}$ and the small value of its optical rotation, it is not reliable to determine the absolute configuration of wickerol A solely based on the optical rotation. Therefore, ECD spectra of synthetic and natural wickerol A were studied. 


\section{ECD spectra of synthetic and natural wickerols A and B}

sample name: synthetic wickerol A (1)

concentration: $\mathrm{C}=0.75 \mathrm{mg} / \mathrm{mL}$ in $\mathrm{CH}_{3} \mathrm{CN}$

wavelength range: $180-400 \mathrm{~nm}$

CD spectrum:

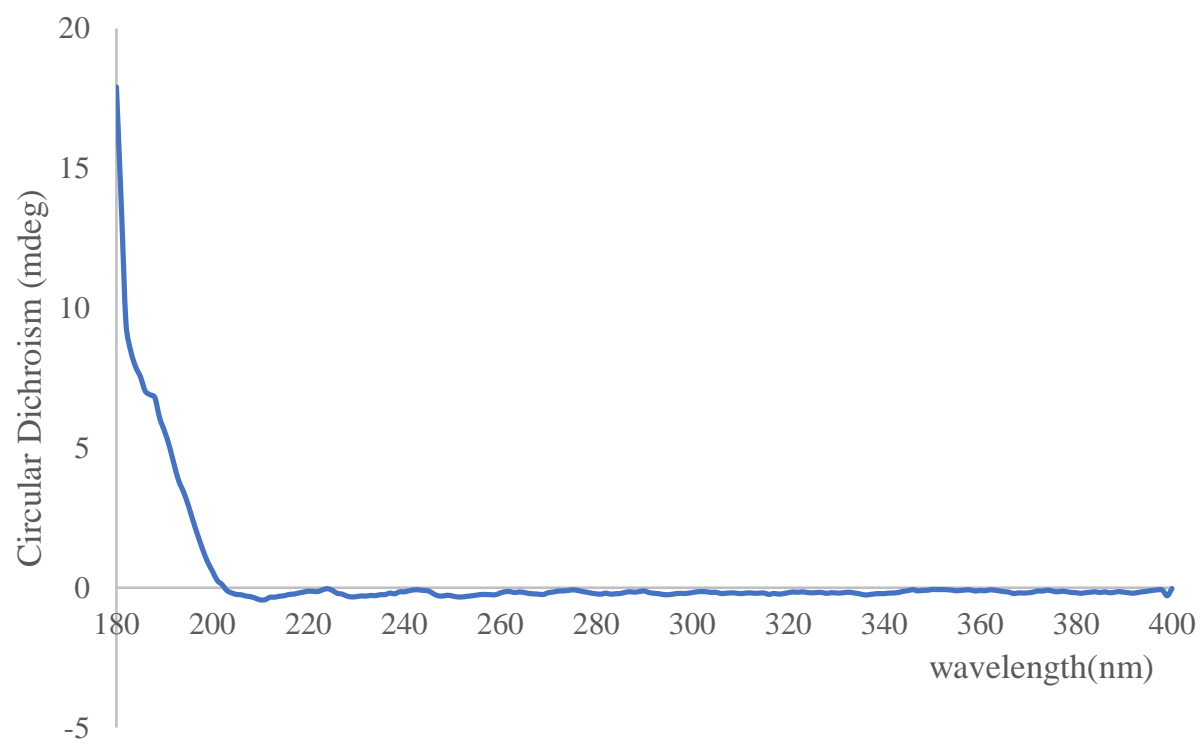

UV spectrum:

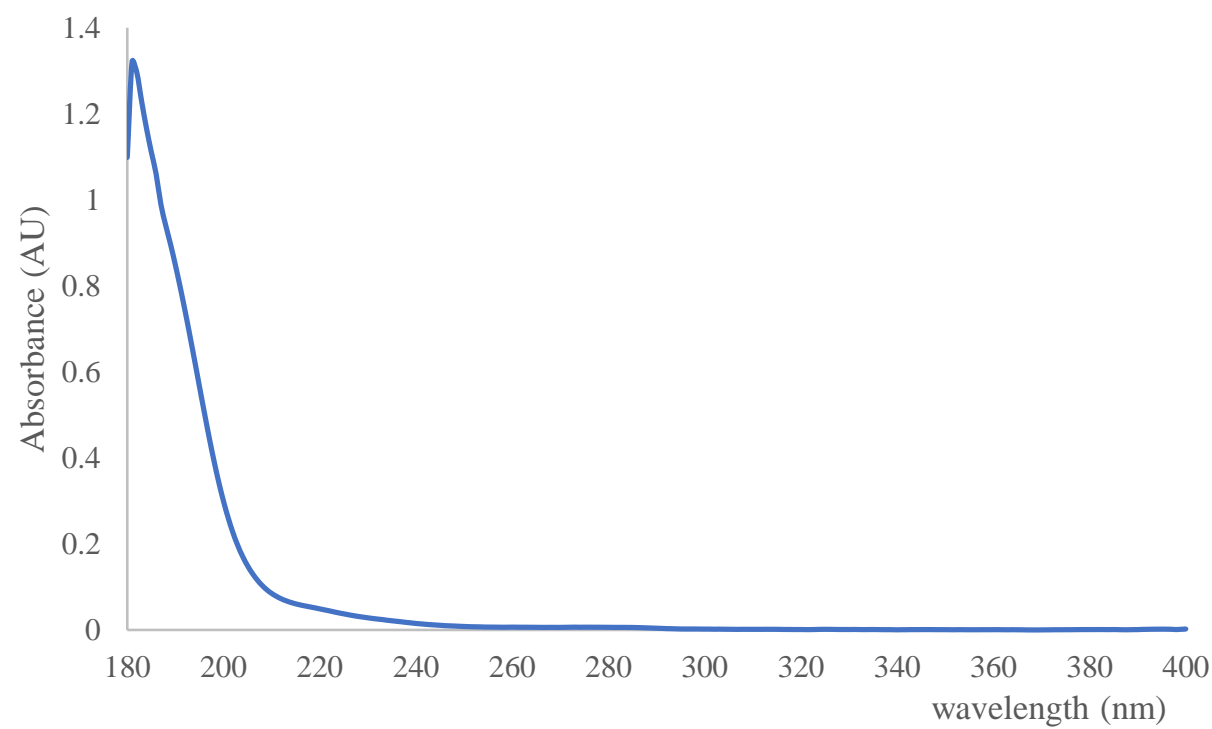


sample name: synthetic wickerol A (1)

concentration: $\mathrm{C}=0.38 \mathrm{mg} / \mathrm{mL}$ in $\mathrm{CH}_{3} \mathrm{CN}$

wavelength range: $180-300 \mathrm{~nm}$

CD spectrum:

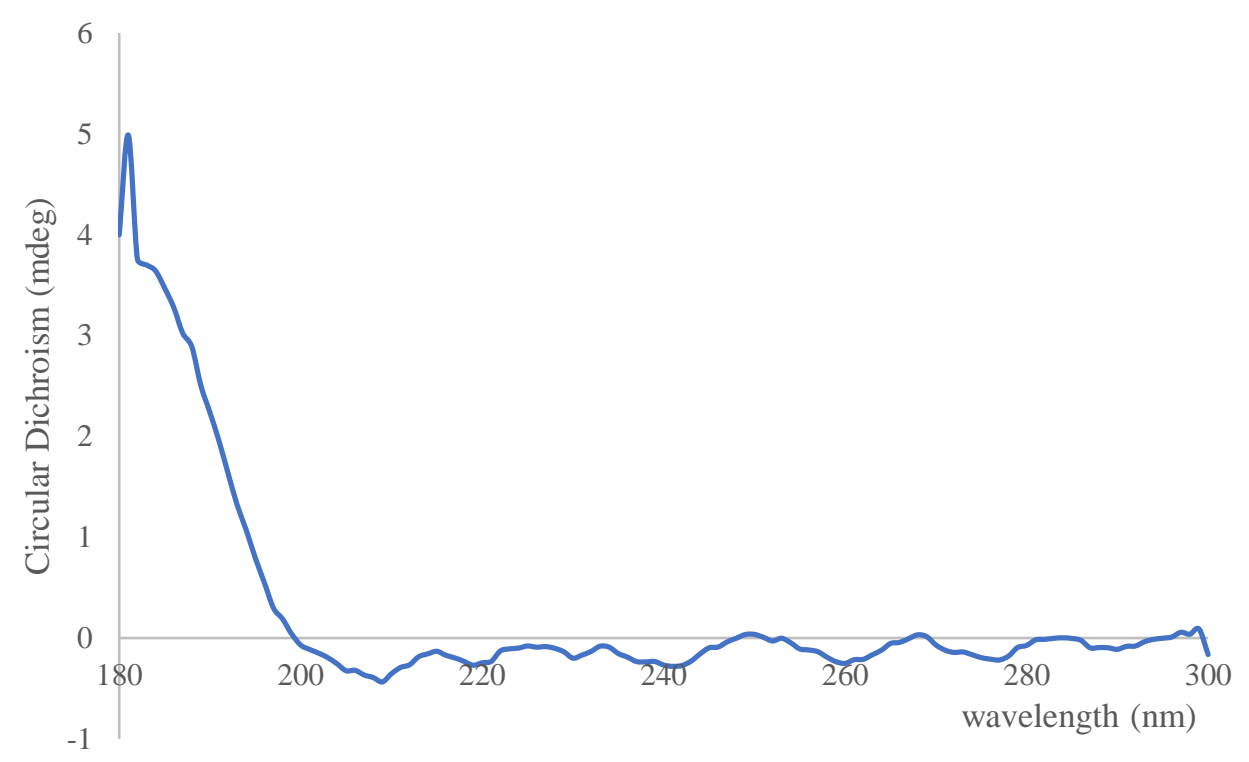

UV spectrum:

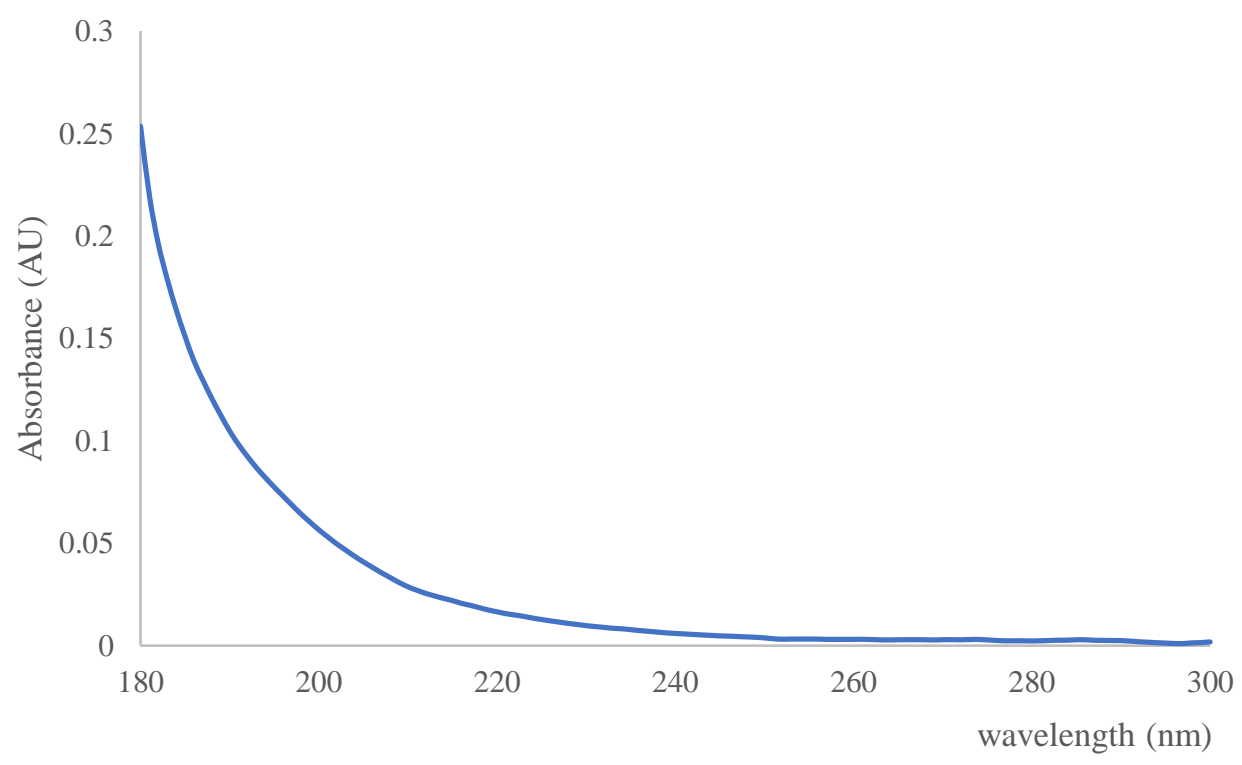


sample name: natural wickerol A (1) (isolated by Prof. Ji and co-workers from the culture of the marine red alga-epiphytic fungus Trichoderma koningiopsis $\mathrm{Y} 10-2)^{7}$

concentration: $\mathrm{C}=0.38 \mathrm{mg} / \mathrm{mL}$ in $\mathrm{CH}_{3} \mathrm{CN}$

wavelength range: $180-400 \mathrm{~nm}$

CD spectrum:

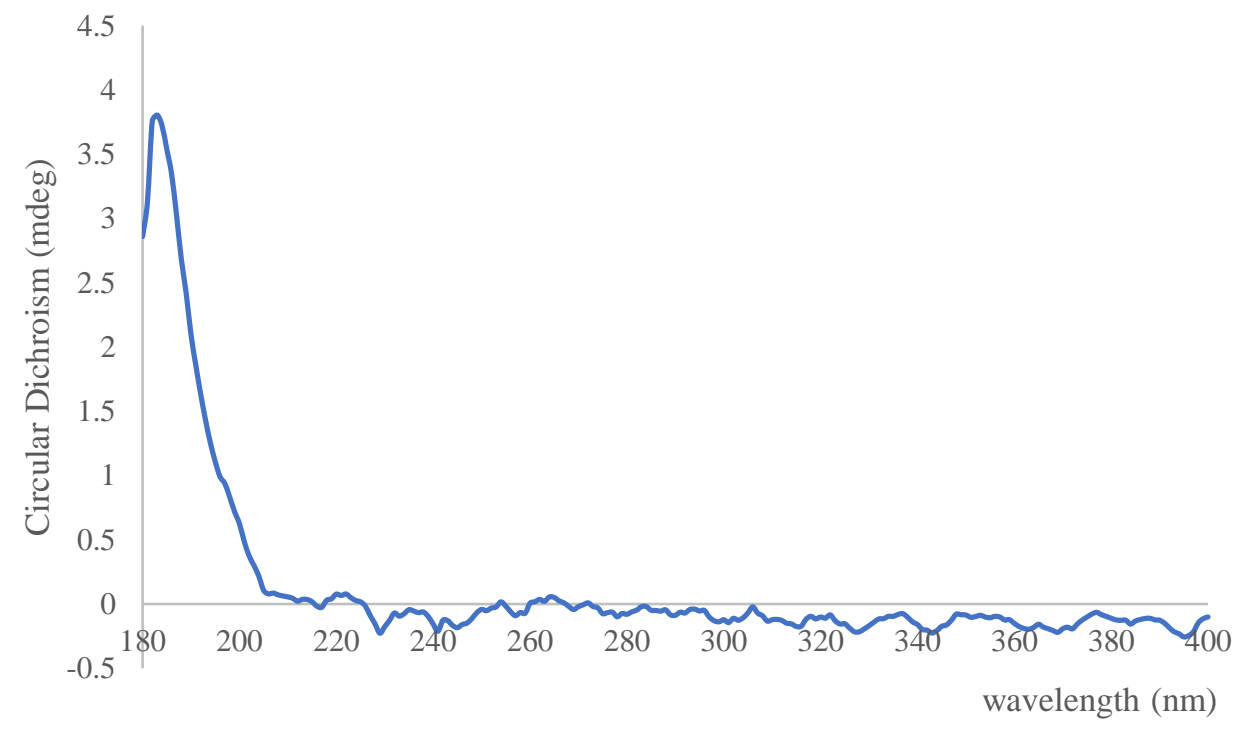

UV spectrum:

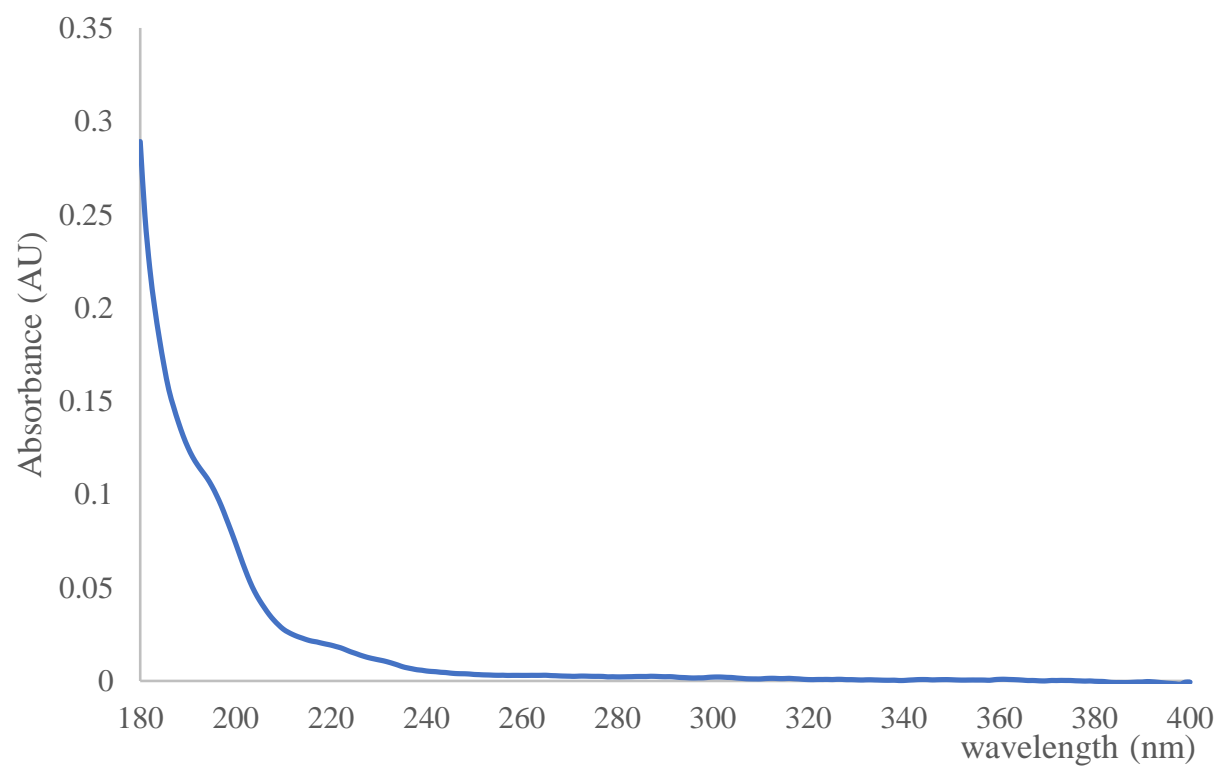


The ECD spectra of Ji's natural and our synthetic wickerol A in MeCN

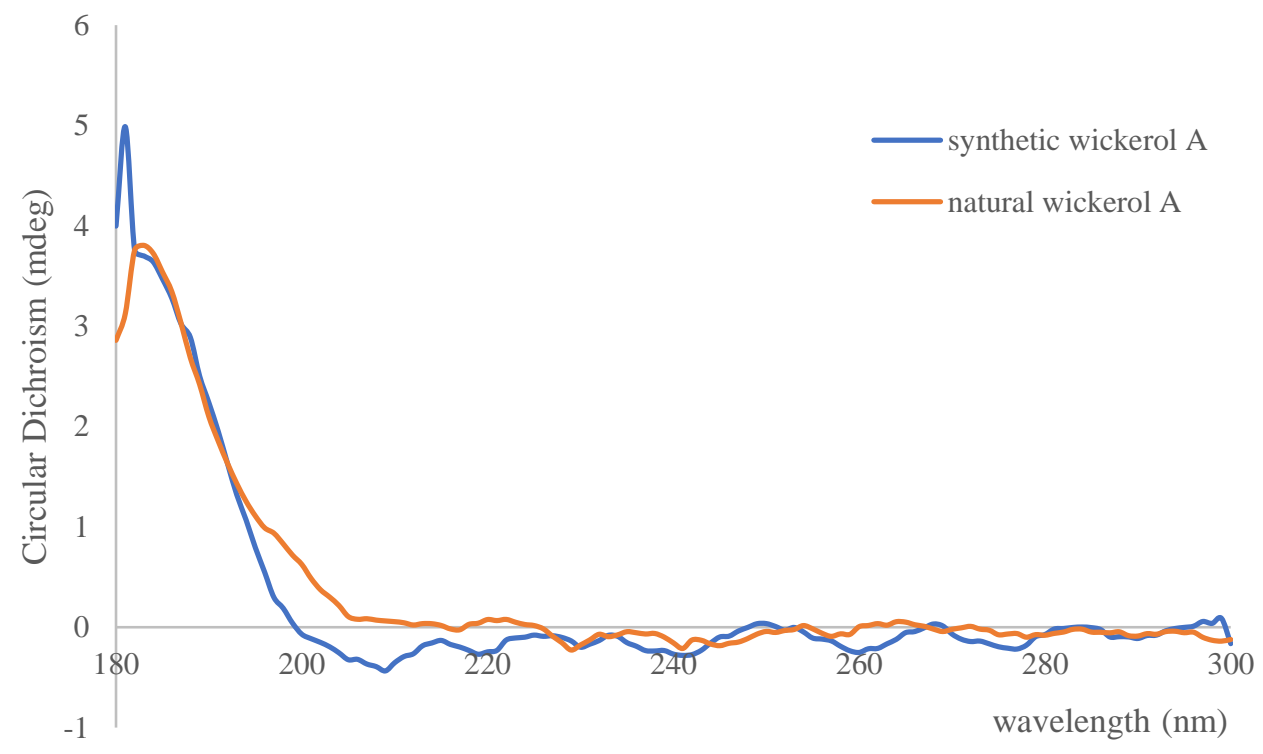

The ECD spectrum of Shiomi's isolated wickerol $\mathrm{A}^{5}$ in $\mathrm{MeCN}(0.54 \mathrm{mg} / \mathrm{mL})$ (measured and kindly provided by Prof. Kazuro Shiomi and his associate Prof. Dr. Masato Iwatsuki).

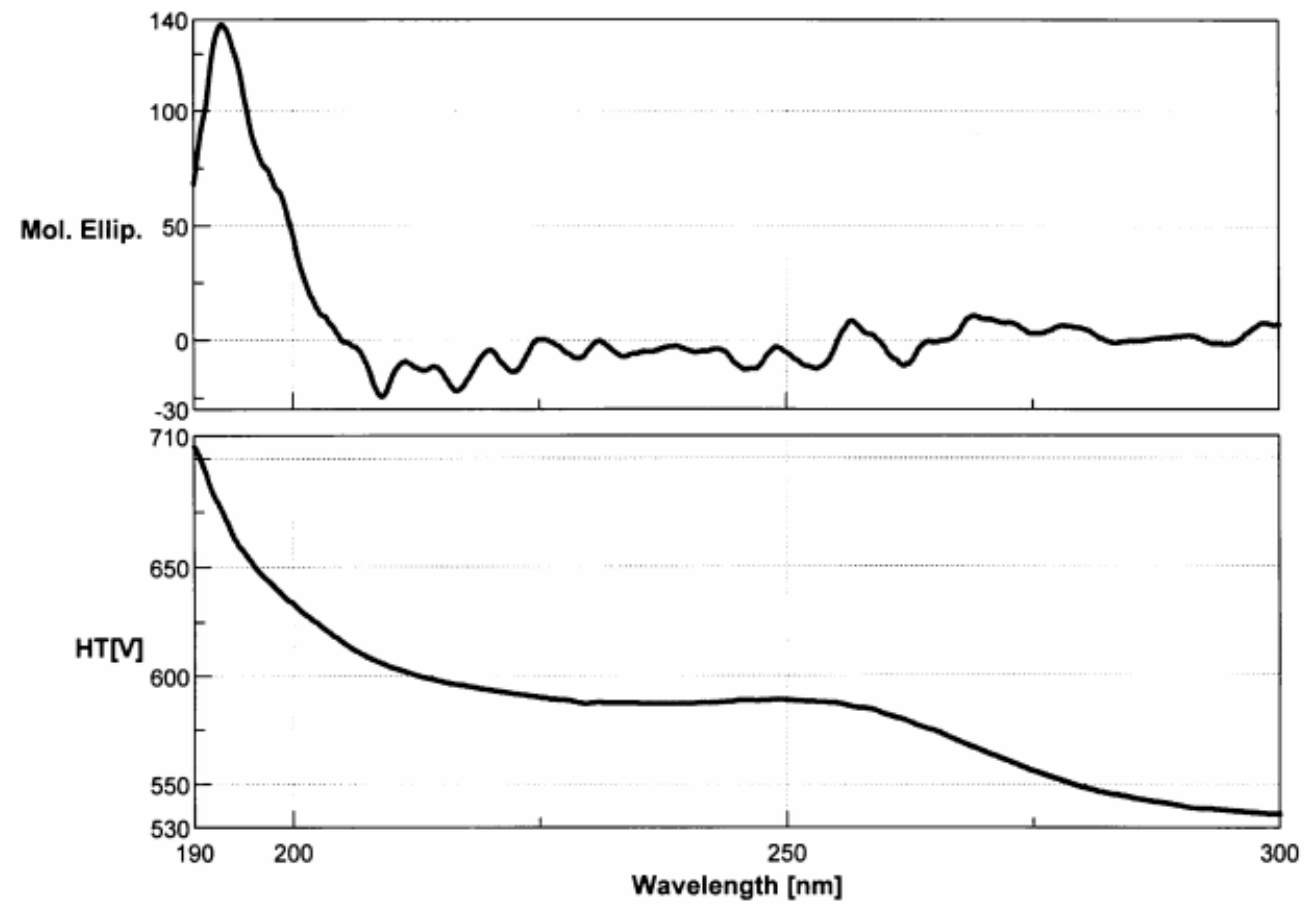

The ECD spectra of natural and synthetic wickerol A clearly suggests that synthetic wickerol A has the same absolute configuration with the natural sample. 
sample name: synthetic wickerol B (2)

concentration: $\mathrm{C}=2.00 \mathrm{mg} / \mathrm{mL}$ in $\mathrm{CH}_{3} \mathrm{CN}$

wavelength range: $180-400 \mathrm{~nm}$

CD spectrum:

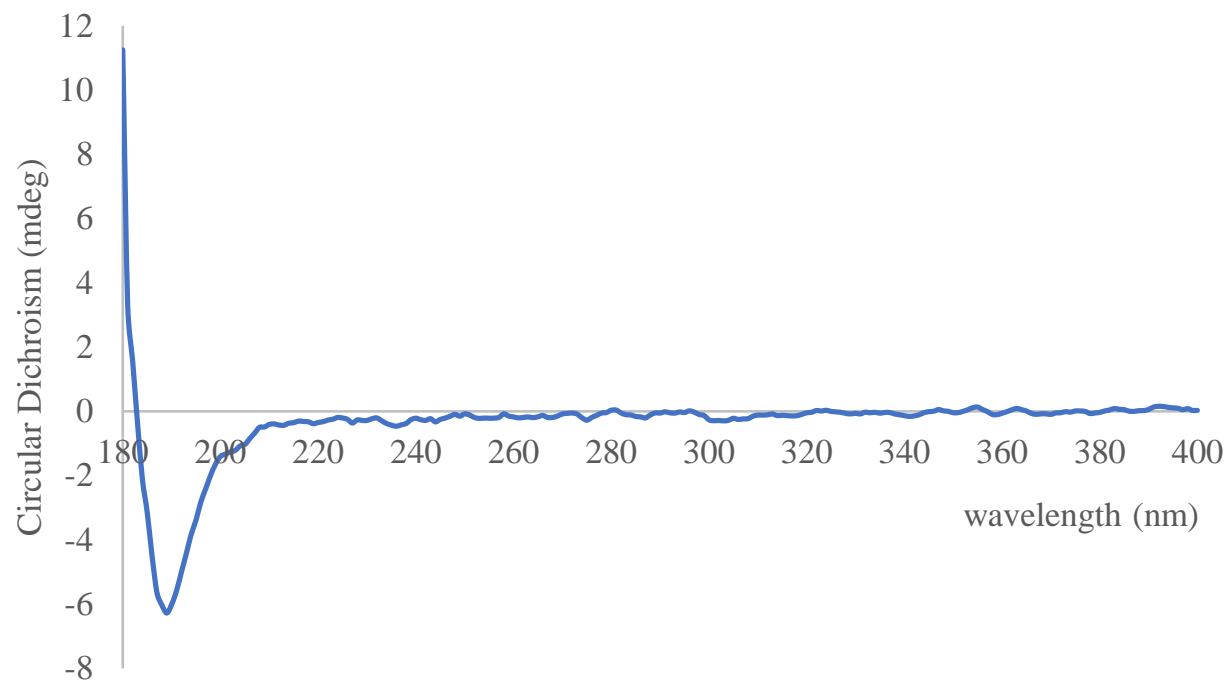

UV spectrum:

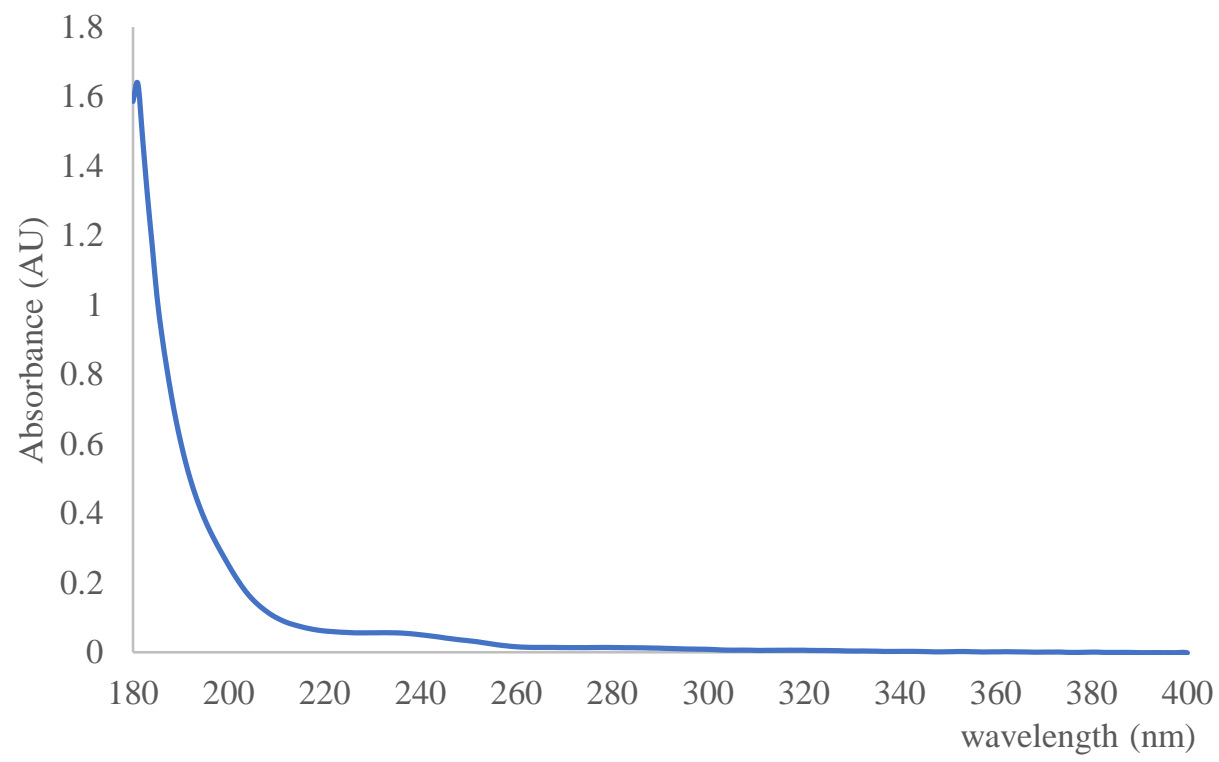


8. X-ray crystallographic data for $3,19,23$

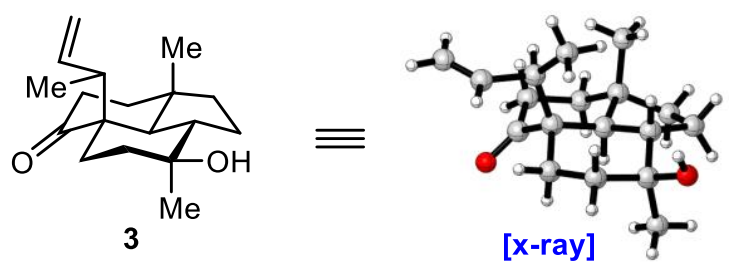

Table S2. Crystal data and structure refinement for CCDC 1960806

Identification code

Empirical formula

Formula weight

Temperature

Wavelength

Crystal system

Space group

Unit cell dimensions

Volume

Z

Density (calculated)

Absorption coefficient

$\mathrm{F}(000)$

Crystal size

Theta range for data collection

Index ranges

Reflections collected

Independent reflections

Completeness to theta $=25.242^{\circ}$

Absorption correction
CCDC 1960806

C18 H28 O2

276.40

293(2) K

$0.71073 \AA$

Orthorhombic

P 212121

$\begin{array}{ll}\mathrm{a}=7.9993(12) \AA & \mathrm{a}=90^{\circ} . \\ \mathrm{b}=12.0989(17) \AA & \mathrm{b}=90^{\circ} . \\ \mathrm{c}=16.417(2) \AA & \mathrm{g}=90^{\circ} .\end{array}$

1588.8(4) $\AA^{3}$

4

$1.155 \mathrm{Mg} / \mathrm{m}^{3}$

$0.073 \mathrm{~mm}^{-1}$

608

$0.170 \times 0.140 \times 0.110 \mathrm{~mm}^{3}$

2.999 to $25.991^{\circ}$.

$-9<=\mathrm{h}<=9,-14<=\mathrm{k}<=12,-20<=\mathrm{l}<=19$

7579

$3098[\mathrm{R}(\mathrm{int})=0.0431]$

$99.6 \%$

Semi-empirical from equivalents 
Max. and min. transmission

Refinement method

Data / restraints / parameters

Goodness-of-fit on $\mathrm{F}^{2}$

Final $\mathrm{R}$ indices [I>2sigma(I)]

$\mathrm{R}$ indices (all data)

Absolute structure parameter

Extinction coefficient

Largest diff. peak and hole
0.7456 and 0.5445

Full-matrix least-squares on $\mathrm{F}^{2}$

3098 / 0 / 186

1.031

$\mathrm{R} 1=0.0511, \mathrm{wR} 2=0.1093$

$\mathrm{R} 1=0.0689, \mathrm{wR} 2=0.1228$

$-0.1(10)$

$0.020(7)$

0.180 and -0.155 e. $\AA^{-3}$ 


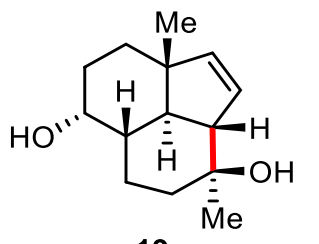

19

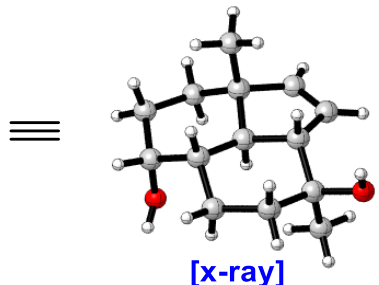

[x-ray]

\section{Table S3. Crystal data and structure refinement for CCDC 1960807}

Identification code

Empirical formula

Formula weight

Temperature

Wavelength

Crystal system

Space group

Unit cell dimensions

Volume

Z

Density (calculated)

Absorption coefficient

$\mathrm{F}(000)$

Crystal size

Theta range for data collection

Index ranges

Reflections collected

Independent reflections

Completeness to theta $=53.594^{\circ}$

Absorption correction

Max. and min. transmission

Refinement method
CCDC 1960807

C14 H22 O2

222.31

$170 \mathrm{~K}$

\section{$1.34139 \AA$}

Monoclinic

P 1211

$$
\begin{array}{ll}
\mathrm{a}=7.53900(10) \AA & \mathrm{a}=90^{\circ} . \\
\mathrm{b}=10.9976(2) \AA & \mathrm{b}=97.4240(10)^{\circ} . \\
\mathrm{c}=15.3730(3) \AA & \mathrm{g}=90^{\circ} .
\end{array}
$$

1263.90(4) $\AA^{3}$

4

$1.168 \mathrm{Mg} / \mathrm{m}^{3}$

$0.383 \mathrm{~mm}^{-1}$

488

$0.08 \times 0.05 \times 0.03 \mathrm{~mm}^{3}$

5.048 to $60.837^{\circ}$.

$-7<=\mathrm{h}<=9,-14<=\mathrm{k}<=14,-19<=\mathrm{l}<=19$

20519

$5771[\mathrm{R}(\mathrm{int})=0.0315]$

$98.8 \%$

Semi-empirical from equivalents

0.7519 and 0.6341

Full-matrix least-squares on $\mathrm{F}^{2}$ 
Data / restraints / parameters

Goodness-of-fit on $\mathrm{F}^{2}$

Final $\mathrm{R}$ indices [I $>2 \operatorname{sigma}(\mathrm{I})]$

$\mathrm{R}$ indices (all data)

Absolute structure parameter

Extinction coefficient

Largest diff. peak and hole
$5771 / 1 / 300$

0.975

$\mathrm{R} 1=0.0304, \mathrm{wR} 2=0.0836$

$\mathrm{R} 1=0.0305, \mathrm{wR} 2=0.0838$

$0.02(4)$

$\mathrm{n} / \mathrm{a}$

0.240 and -0.150 e. $\AA^{-3}$ 


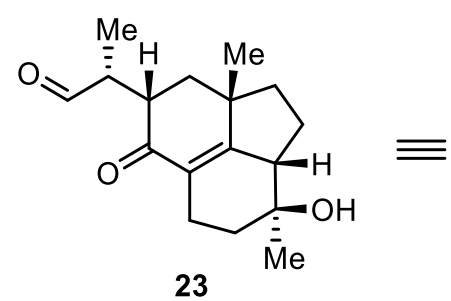

23

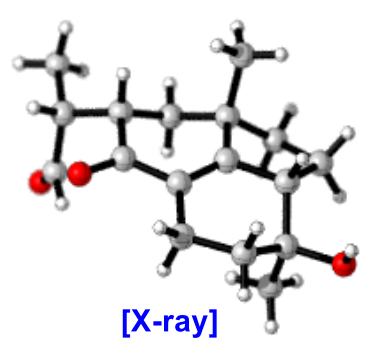

Table S4. Crystal data and structure refinement for CCDC 1960808

Identification code

Empirical formula

Formula weight

Temperature

Wavelength

Crystal system

Space group

Unit cell dimensions

Volume

Z

Density (calculated)

Absorption coefficient

$\mathrm{F}(000)$

Crystal size

Theta range for data collection

Index ranges

Reflections collected

Independent reflections

Completeness to theta $=25.242^{\circ}$

Absorption correction

Max. and min. transmission
CCDC 1960808

C34 H50 O7

570.74

293(2) K

$0.71073 \AA$

Monoclinic

C 2

$\mathrm{a}=32.471(10) \AA \quad \mathrm{a}=90^{\circ}$.

$\mathrm{b}=6.632(2) \AA \quad \mathrm{b}=113.844(9)^{\circ}$.

$\mathrm{c}=16.071(6) \AA \quad \mathrm{g}=90^{\circ}$.

3165.7(18) $\AA^{3}$

4

$1.197 \mathrm{Mg} / \mathrm{m}^{3}$

$0.082 \mathrm{~mm}^{-1}$

1240

$0.170 \times 0.130 \times 0.070 \mathrm{~mm}^{3}$

2.310 to $25.500^{\circ}$.

$-39<=\mathrm{h}<=36,-8<=\mathrm{k}<=8,-12<=\mathrm{l}<=19$

14487

$5586[\mathrm{R}(\mathrm{int})=0.0535]$

$99.8 \%$

Semi-empirical from equivalents

0.7456 and 0.6297 
Refinement method

Data / restraints / parameters

Goodness-of-fit on $\mathrm{F}^{2}$

Final $\mathrm{R}$ indices [I $>2 \operatorname{sigma}(\mathrm{I})]$

$\mathrm{R}$ indices (all data)

Absolute structure parameter

Extinction coefficient

Largest diff. peak and hole
Full-matrix least-squares on $\mathrm{F}^{2}$

$5586 / 30 / 400$

1.044

$\mathrm{R} 1=0.0611, \mathrm{wR} 2=0.1199$

$\mathrm{R} 1=0.1317, \mathrm{wR} 2=0.1530$

$0.9(10)$

$0.0046(7)$

0.214 and -0.187 e. $\AA^{-3}$ 


\section{NMR spectra}

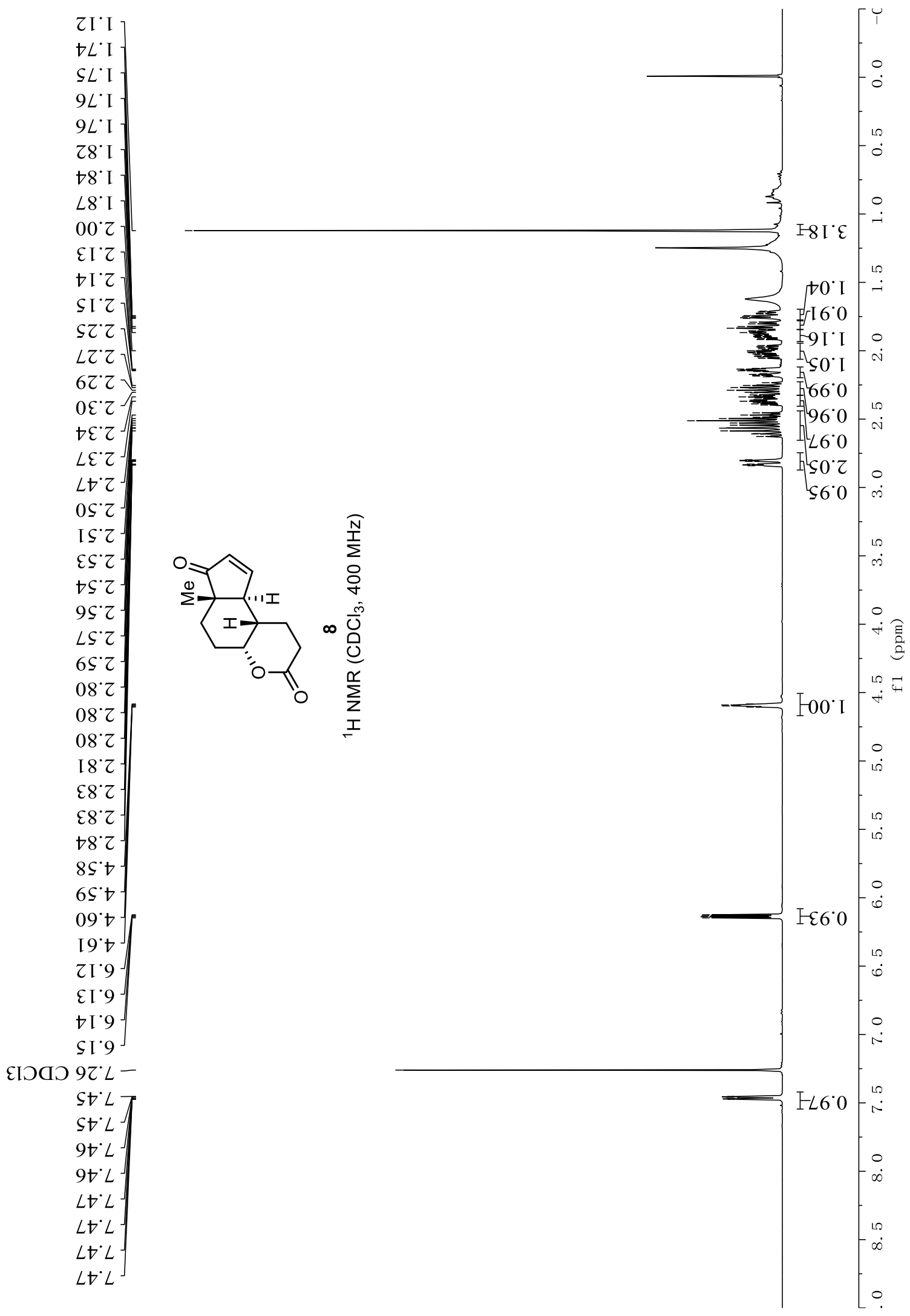




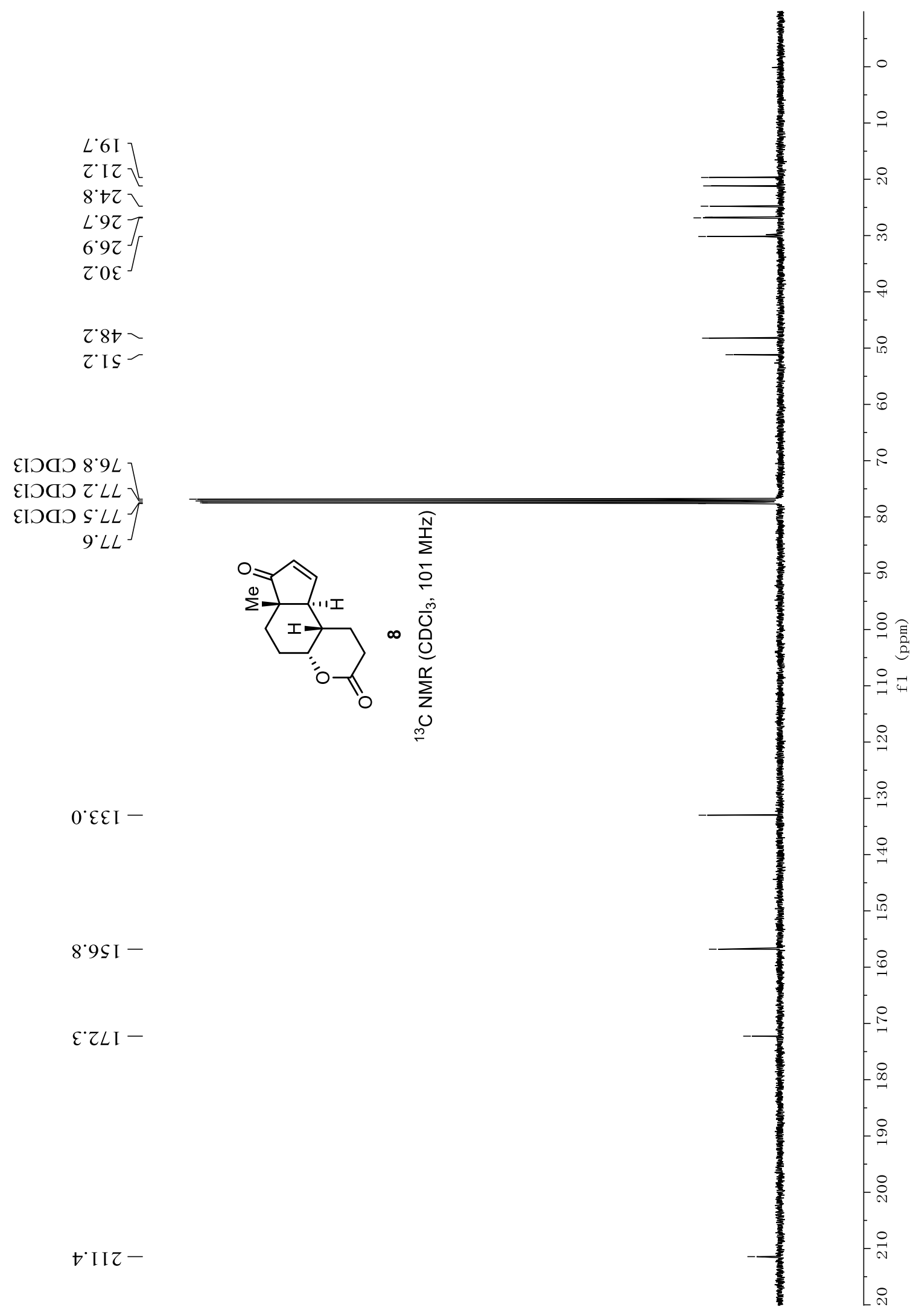




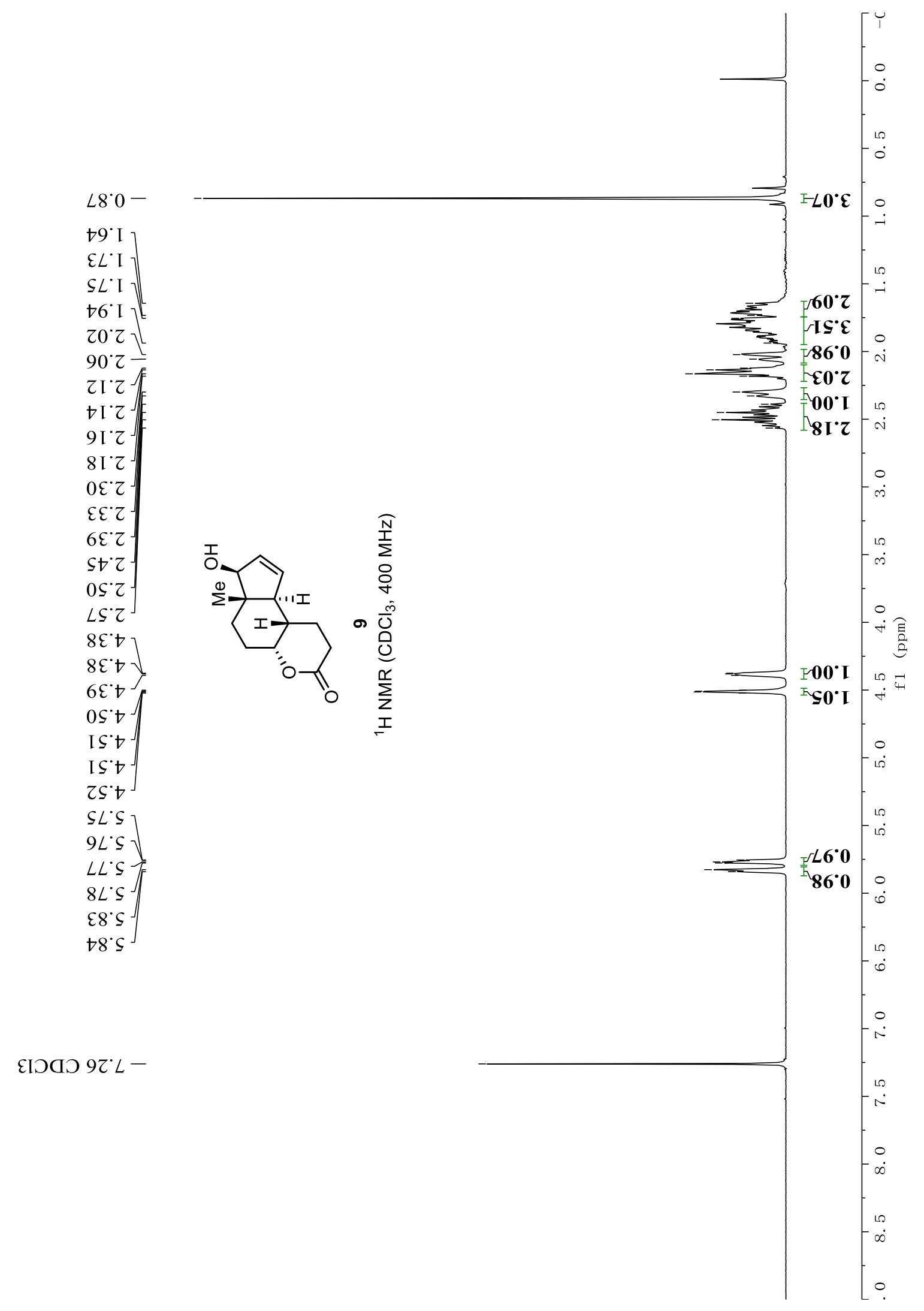



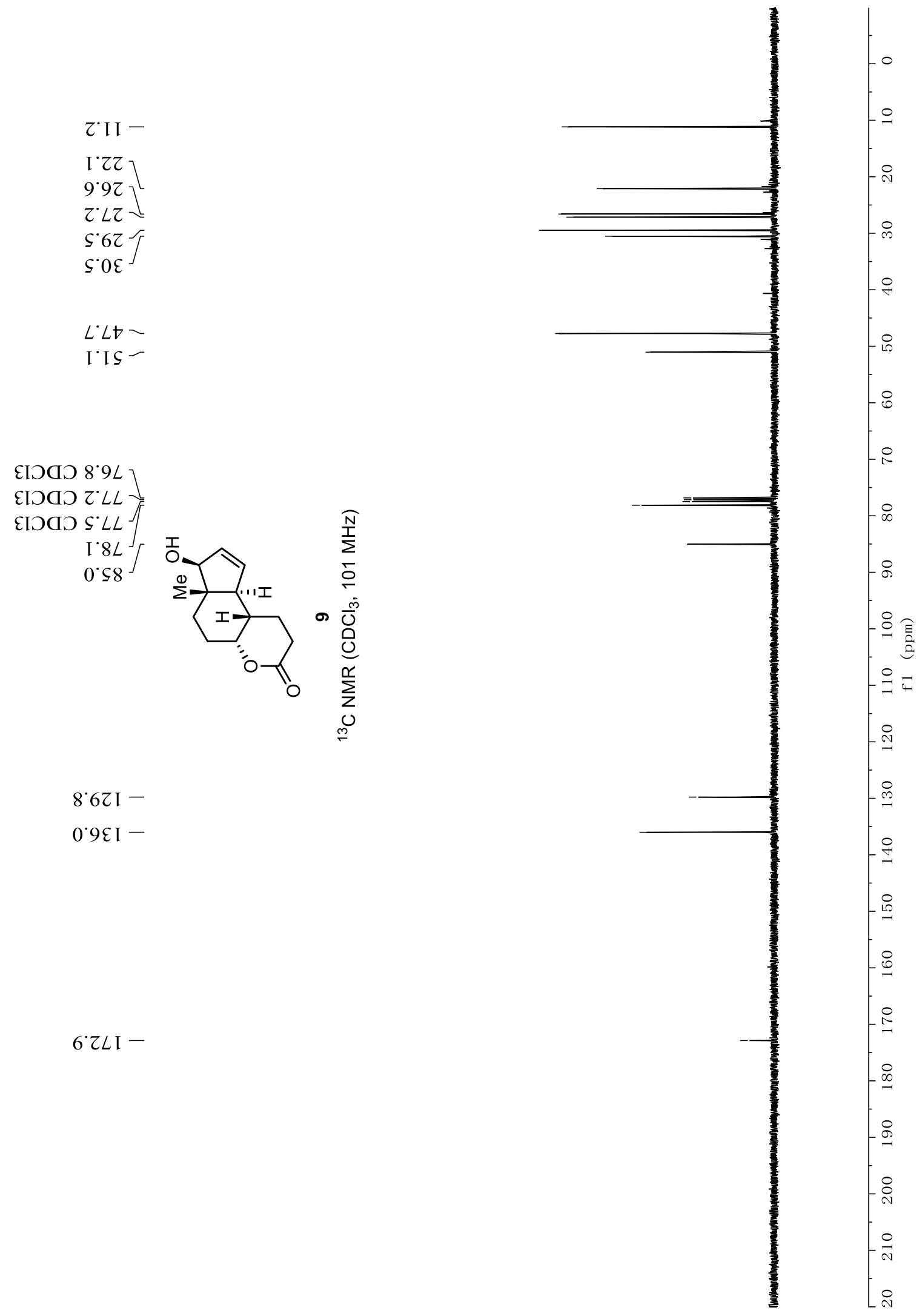

8.6ZI -

$0.9 \varepsilon$ I -

$6.2 L I-$ 


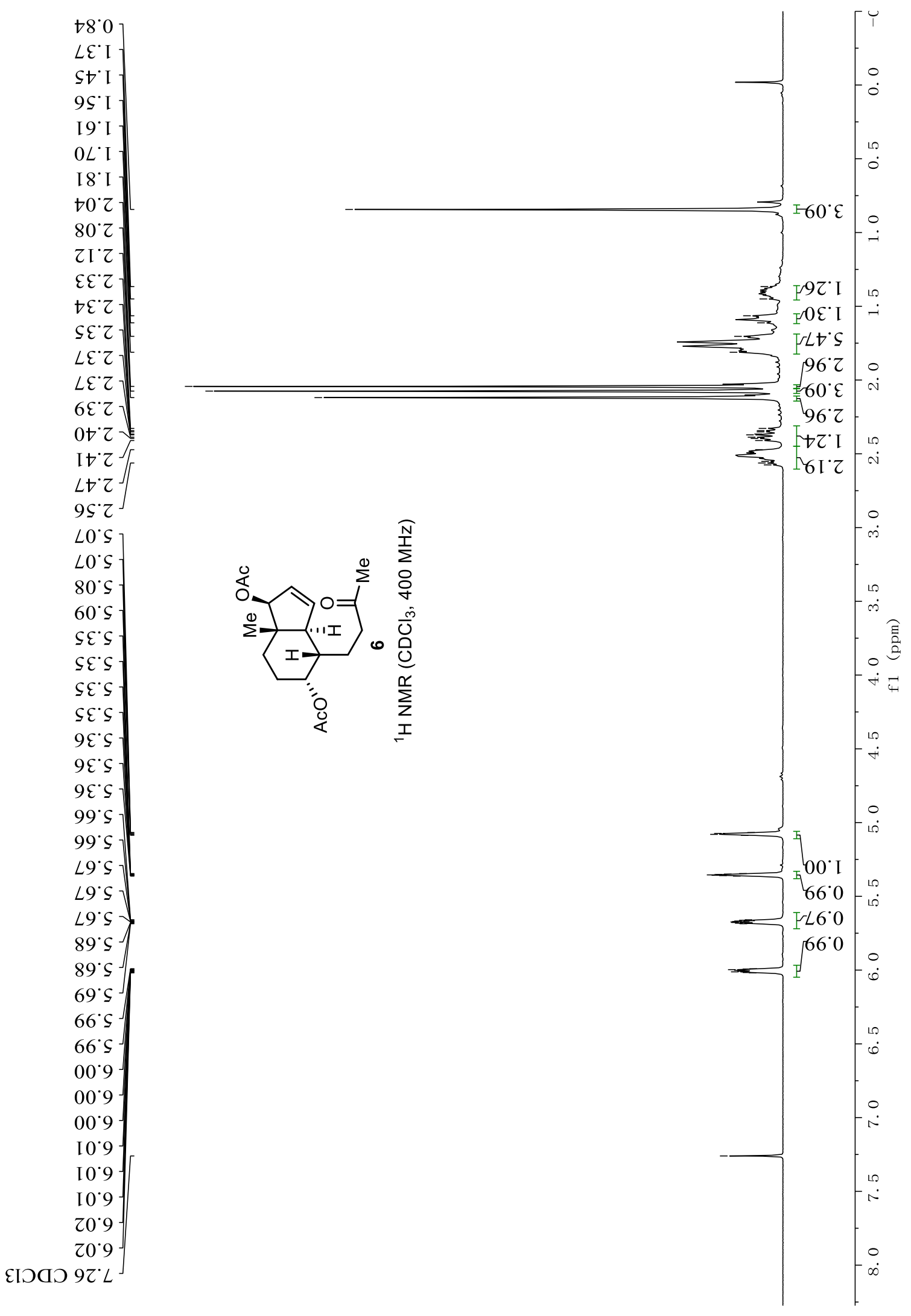



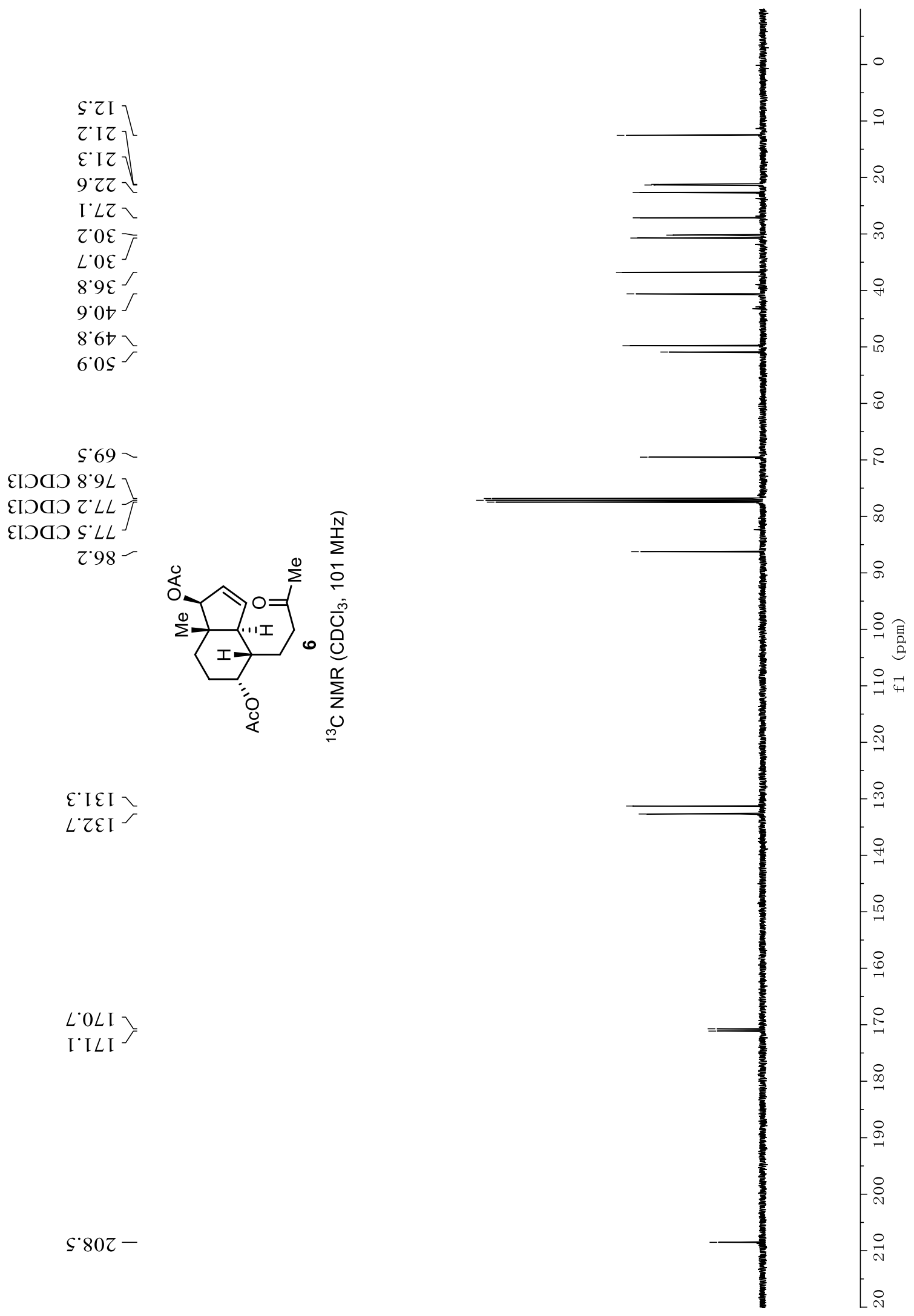


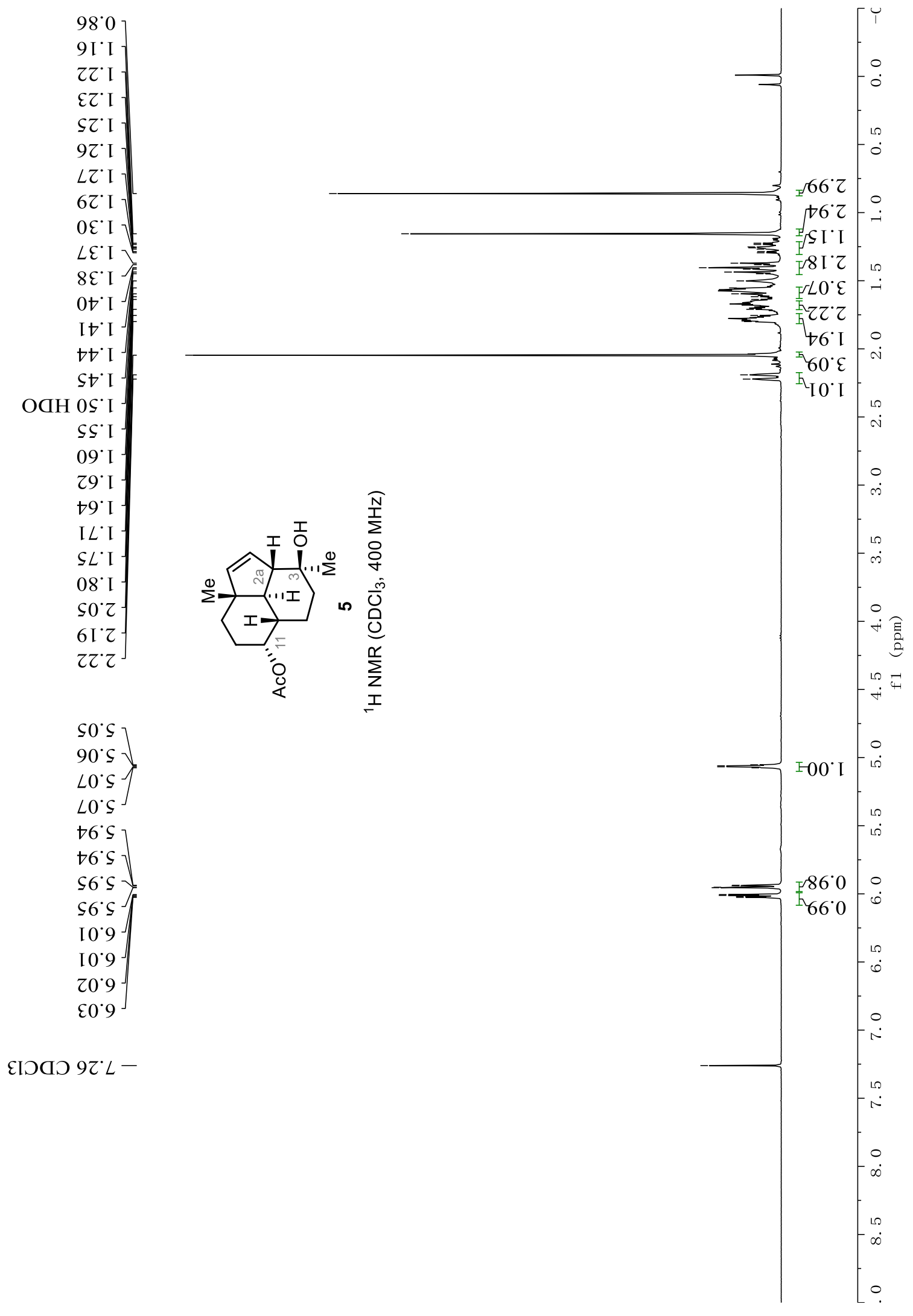




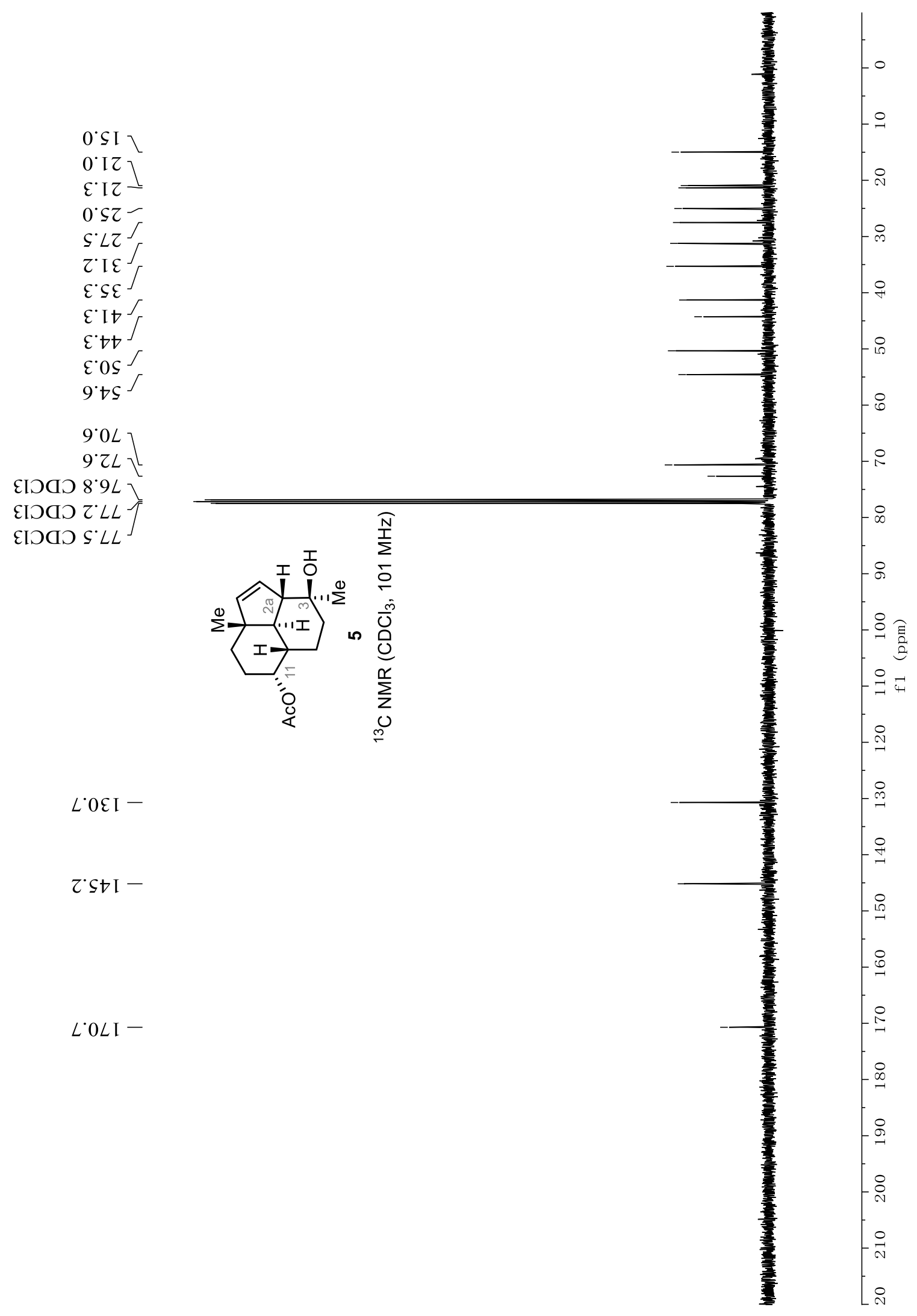




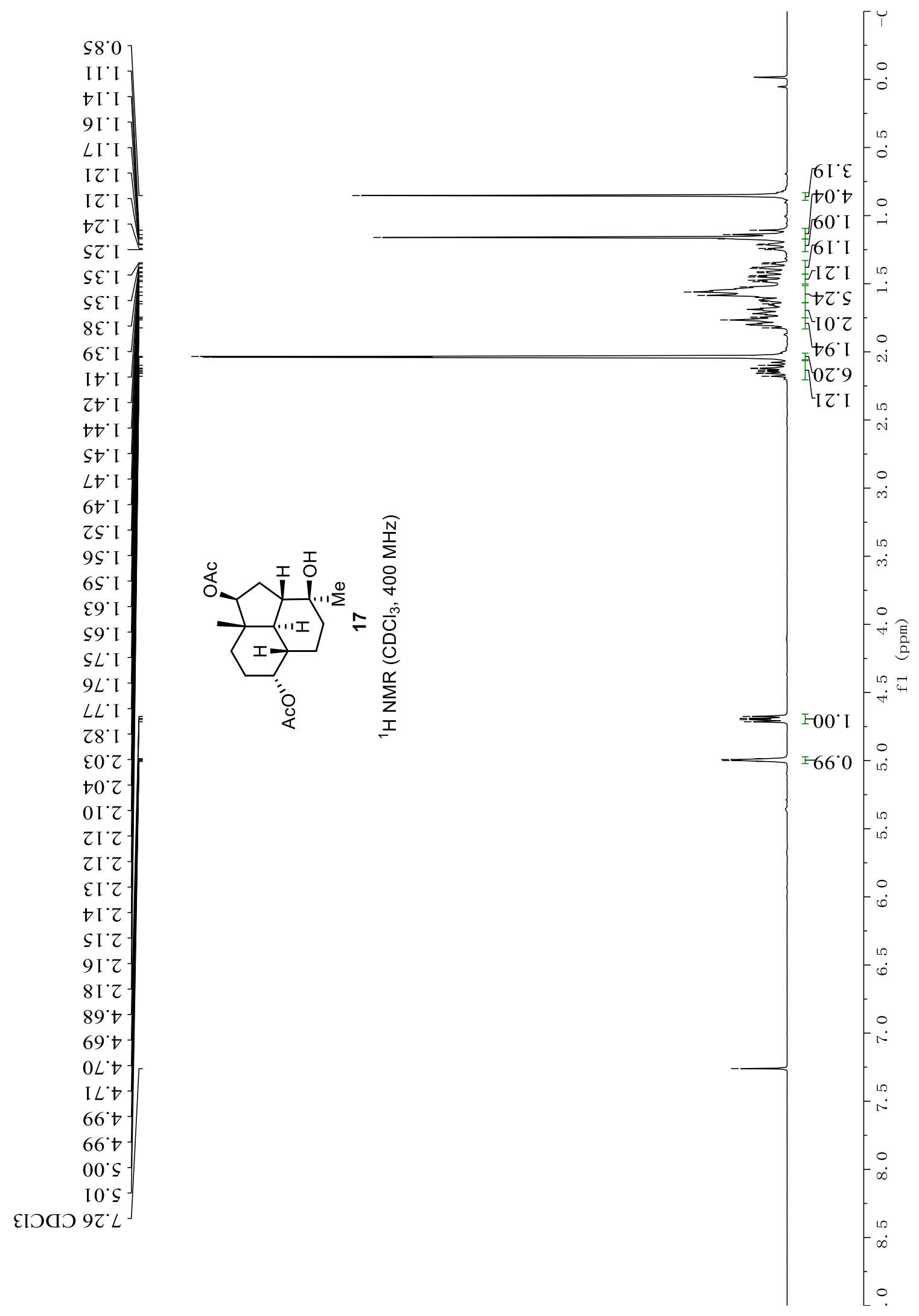



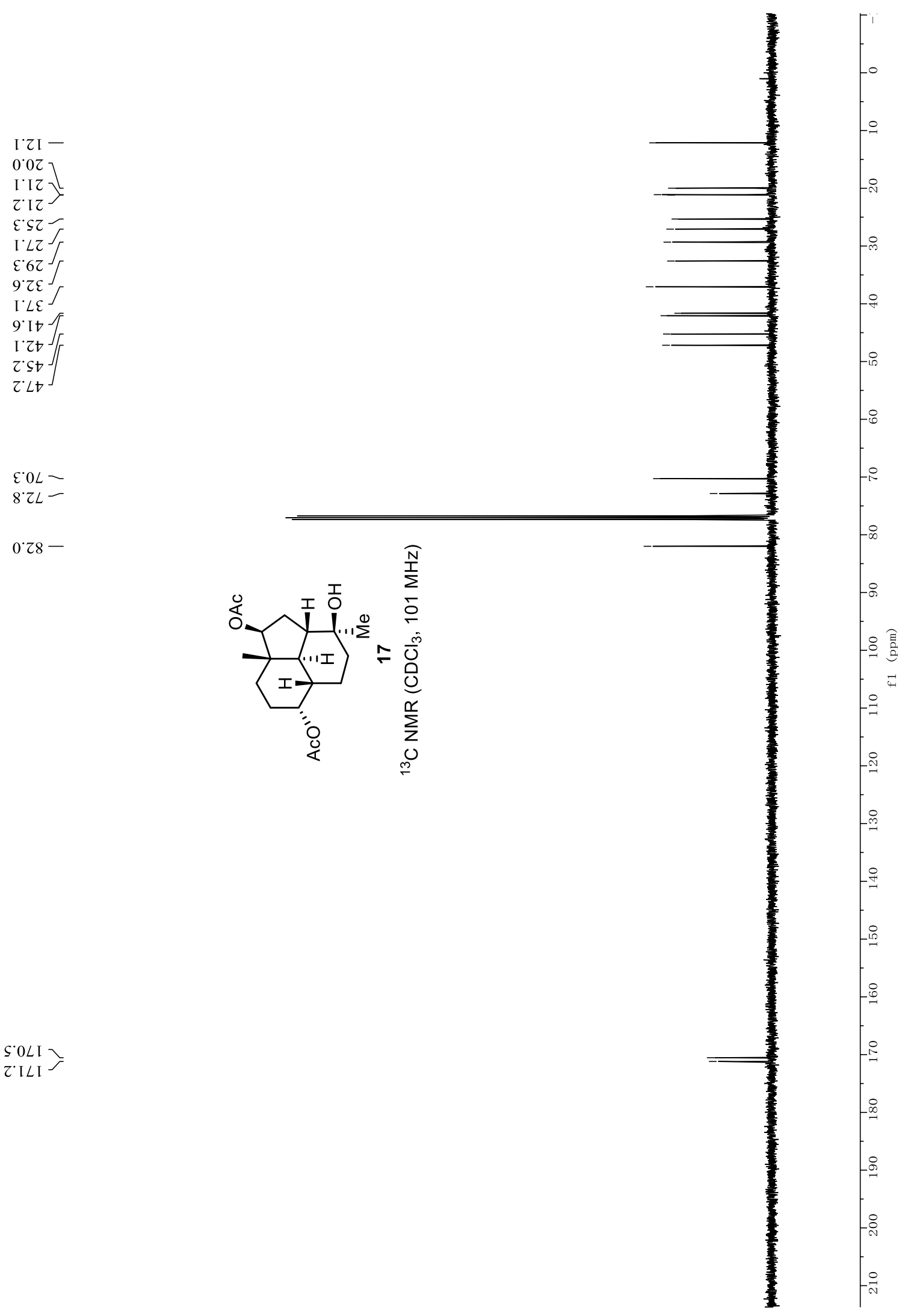

S.0LI
Z'I LI I 

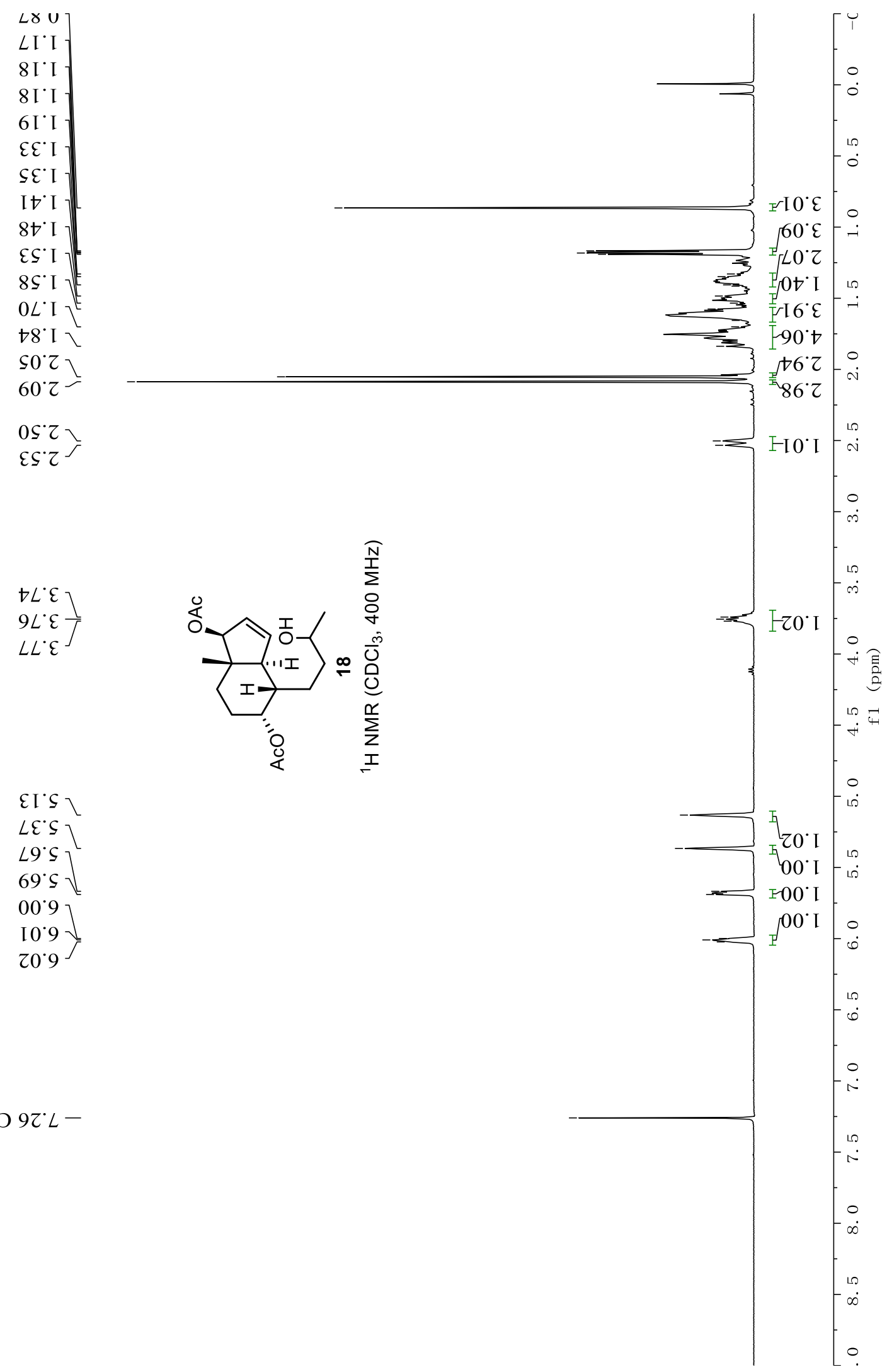

ยเวดว $9{ }^{\circ} L-$ 


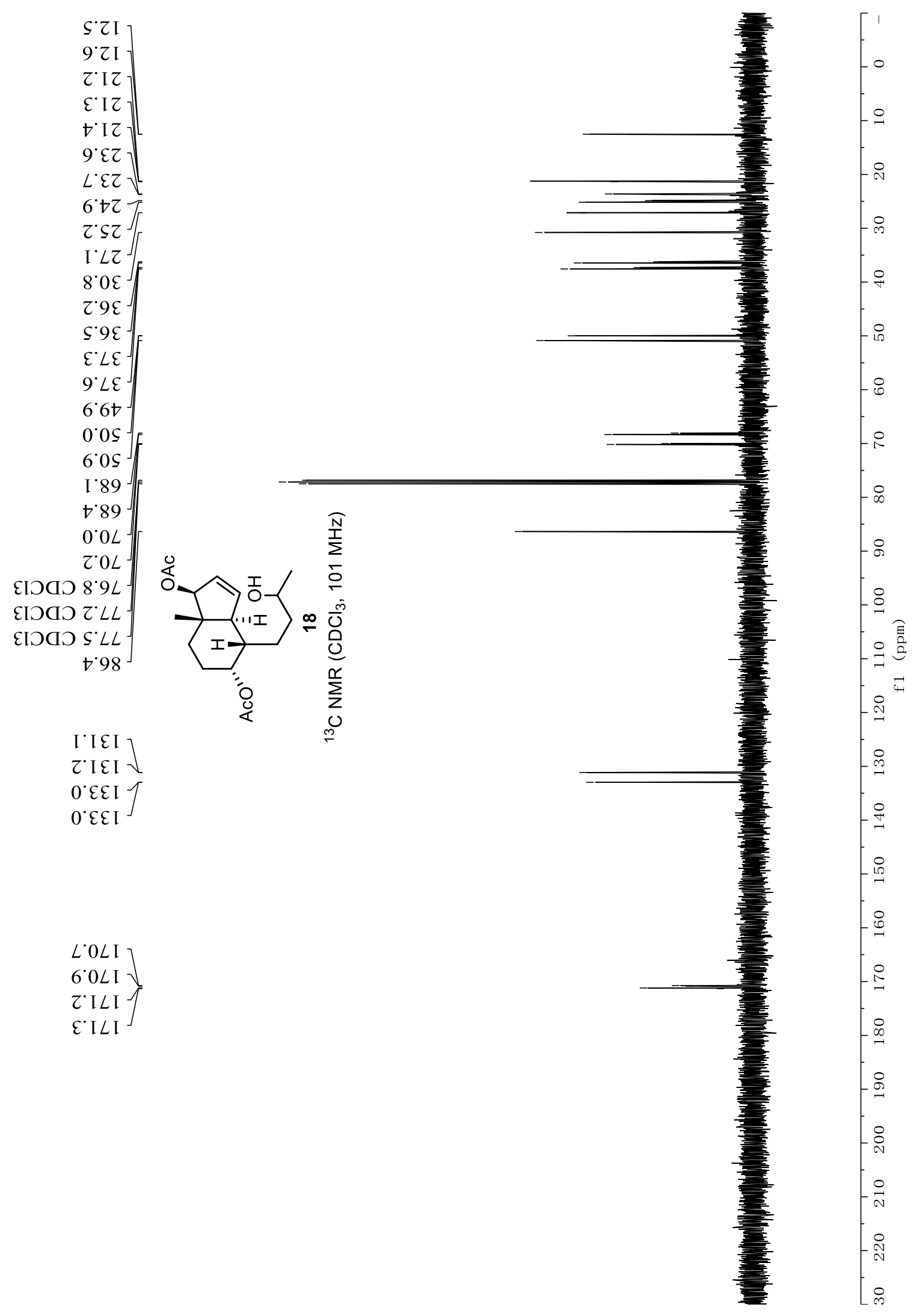




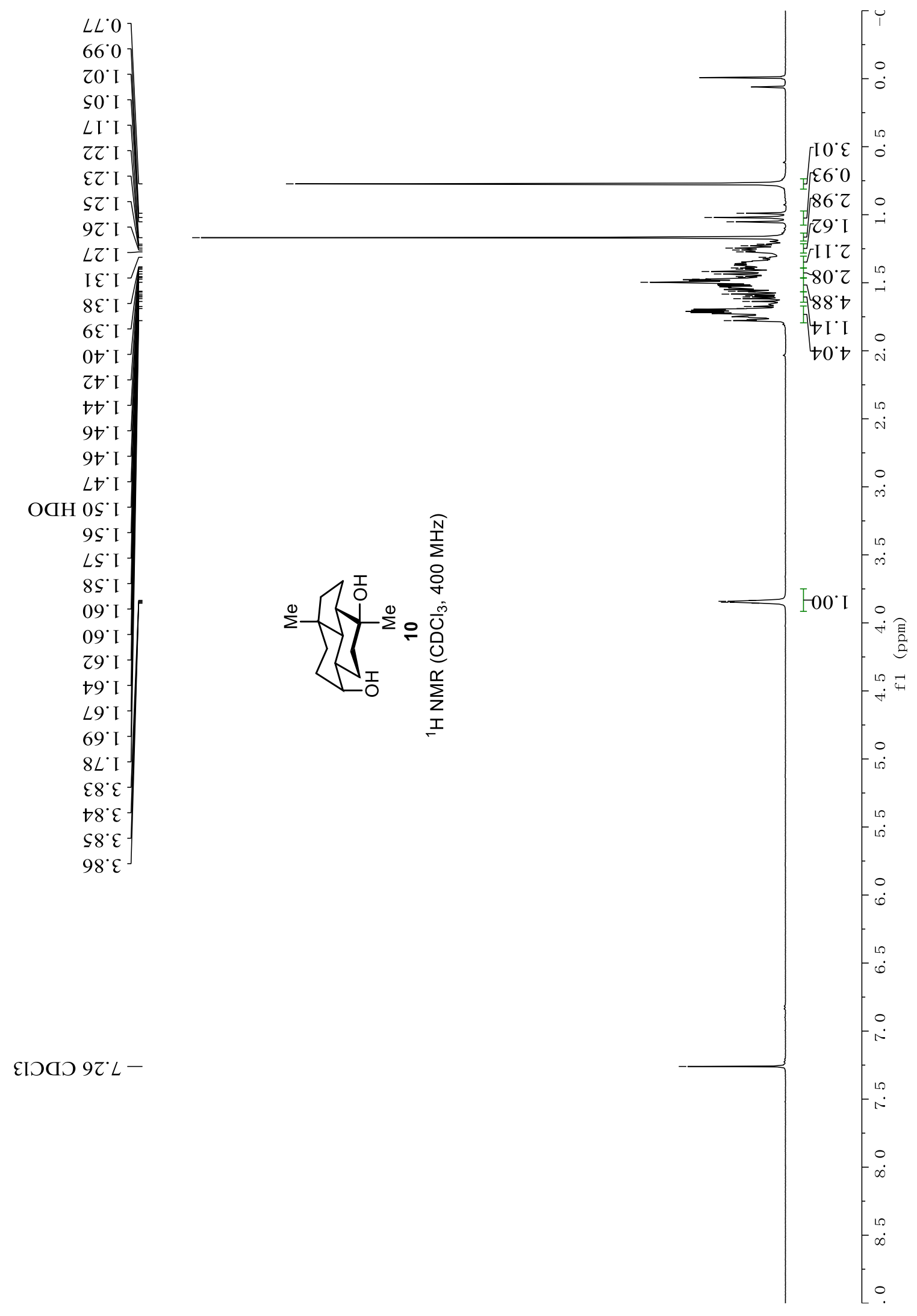




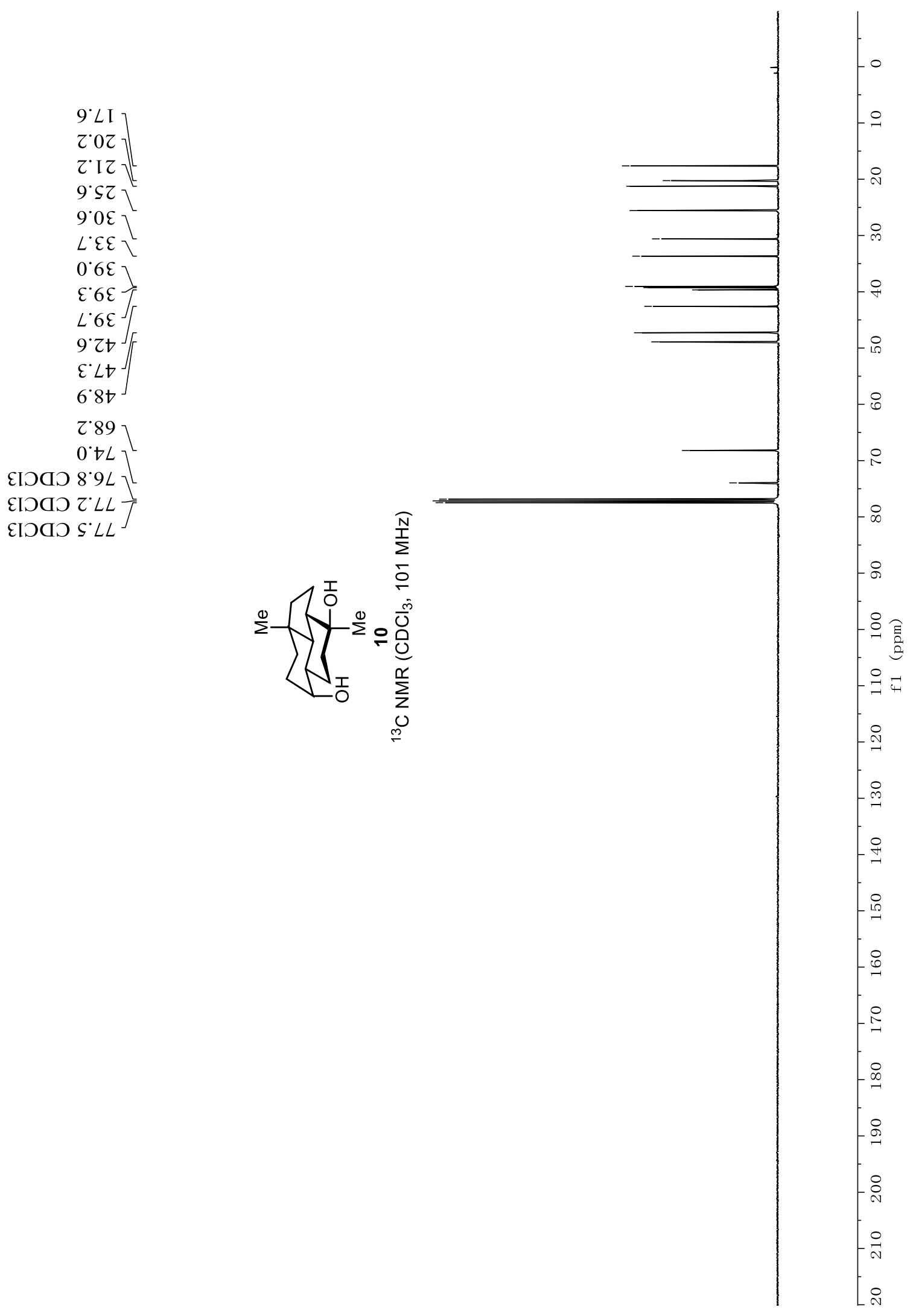




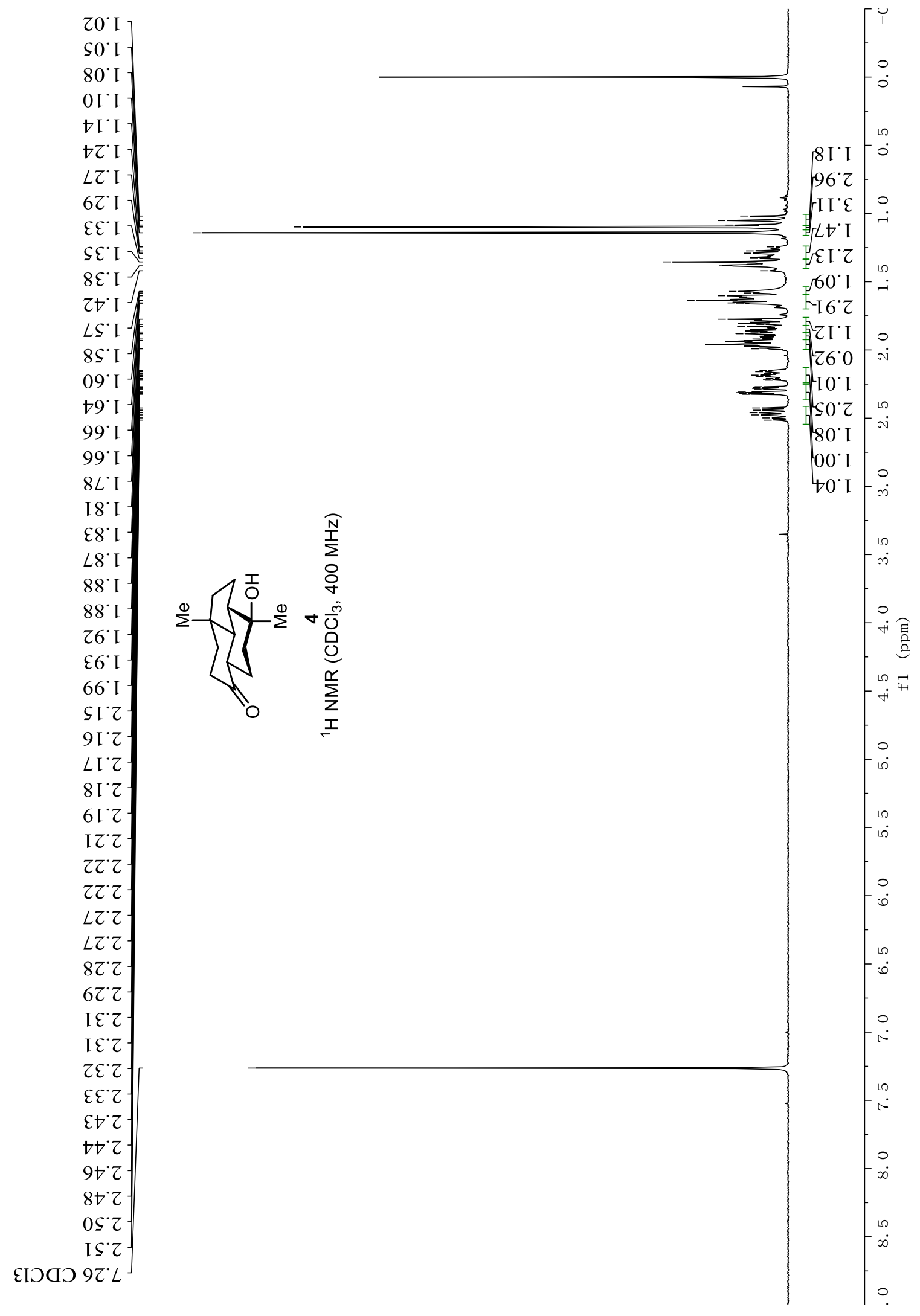



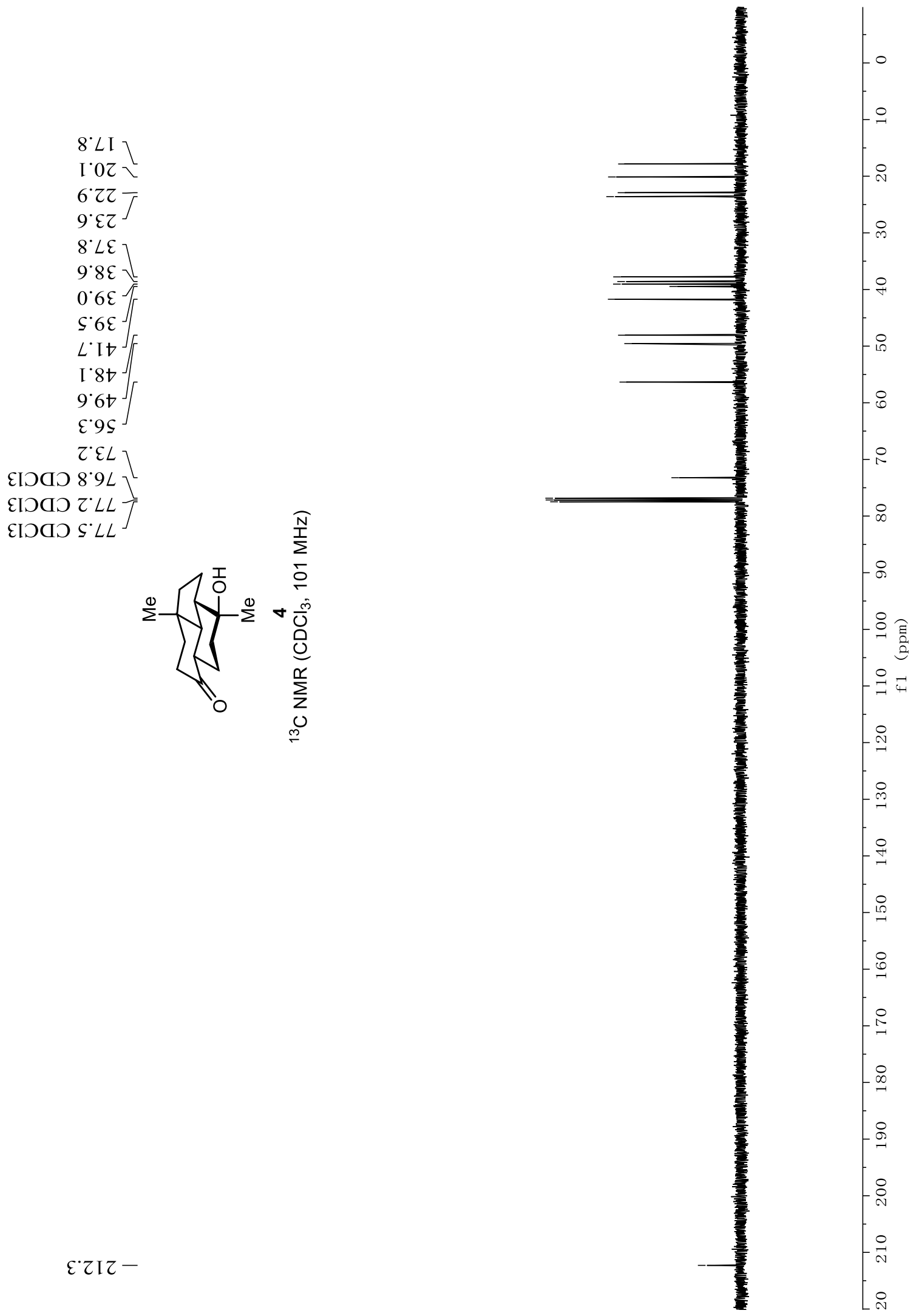


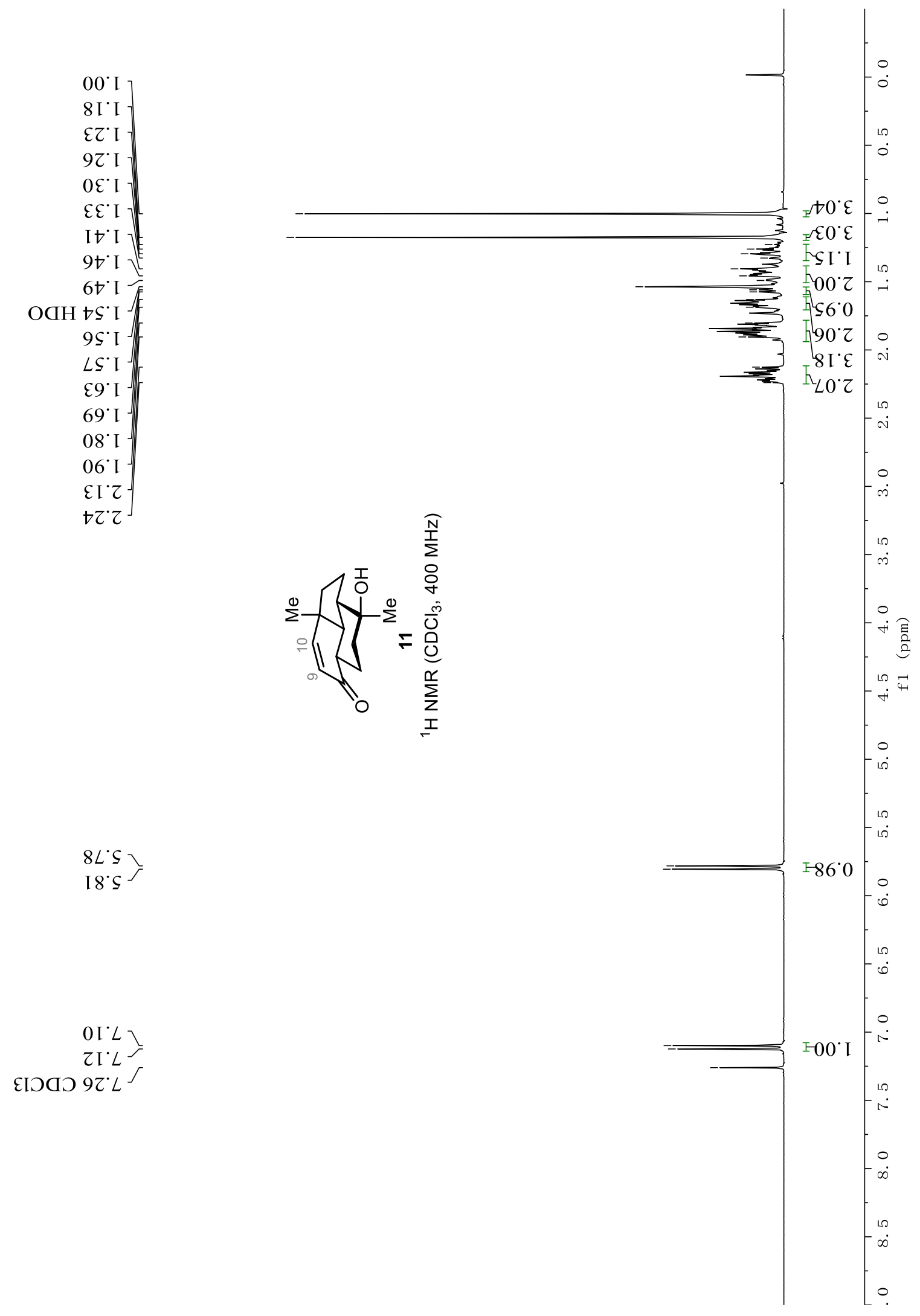



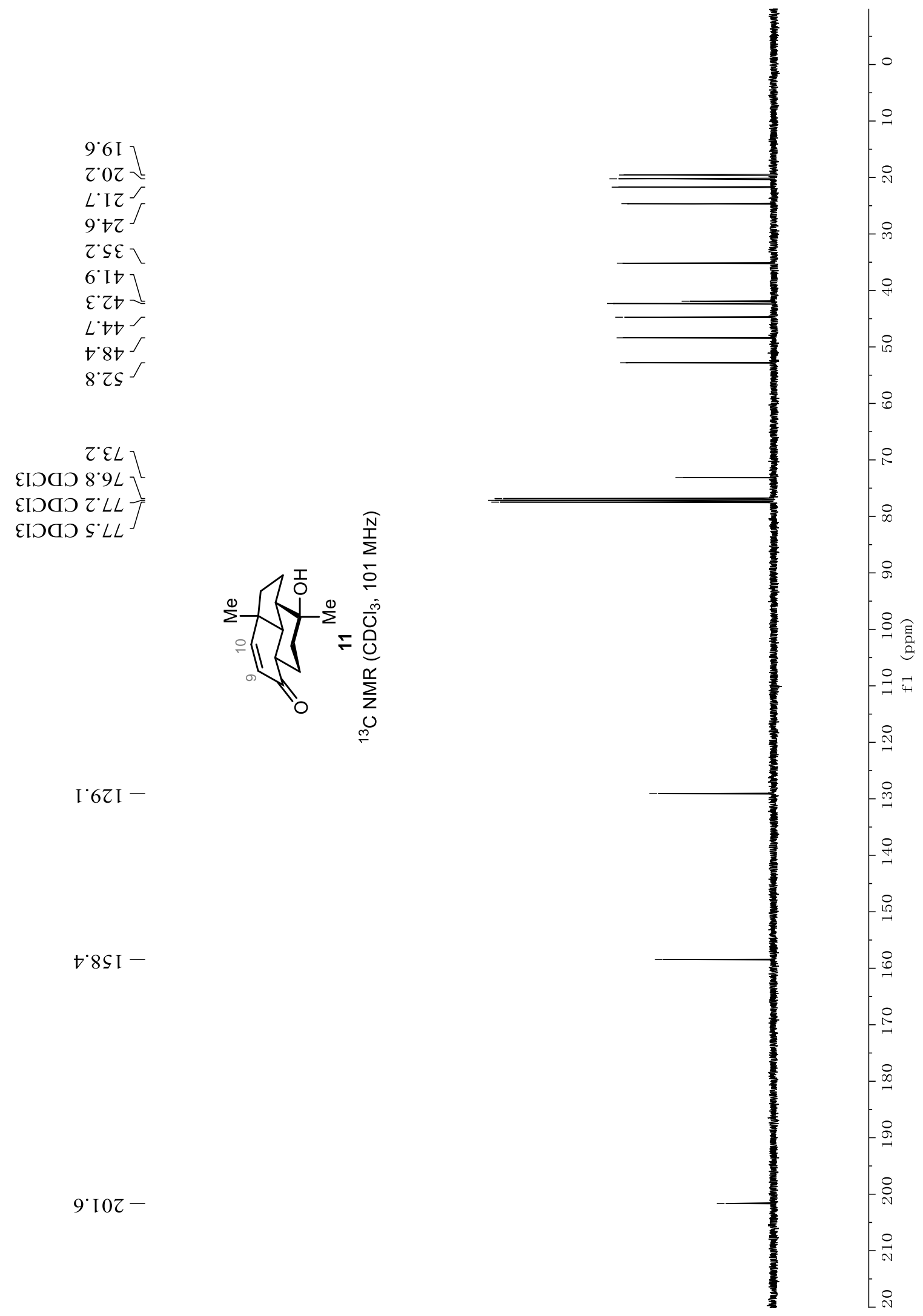


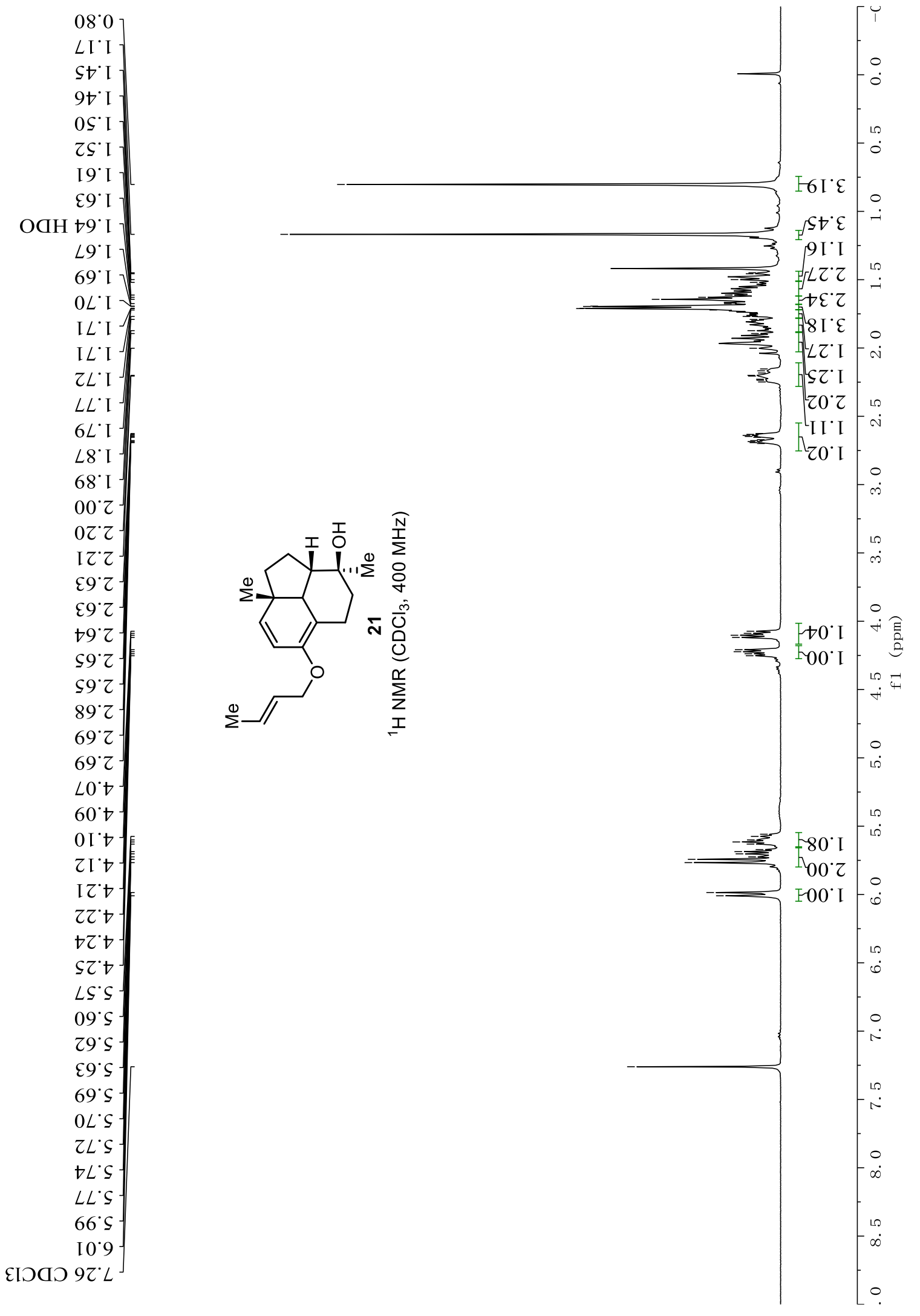



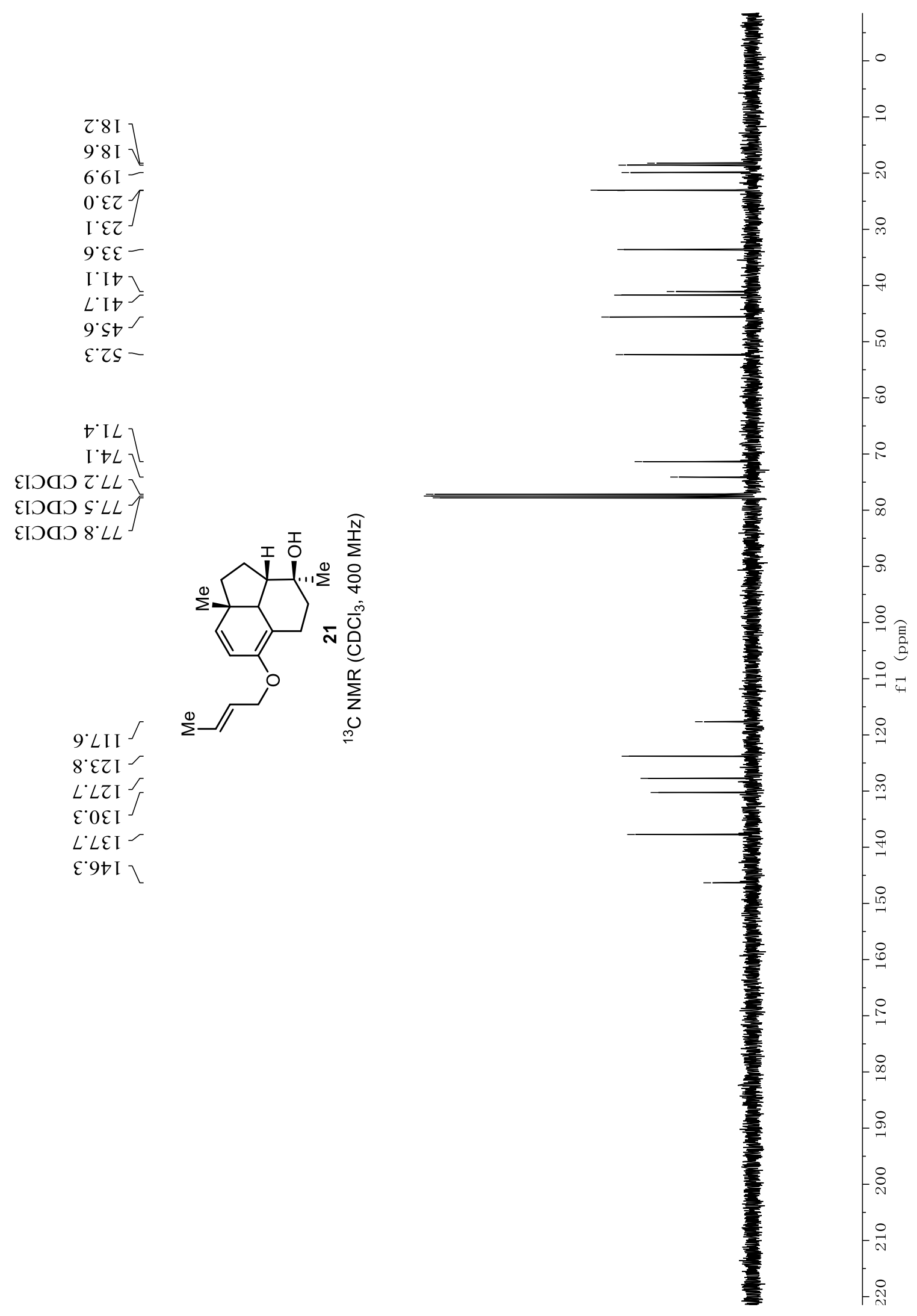


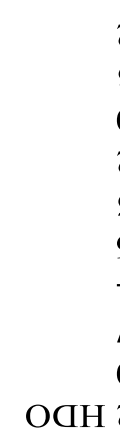

乙 I ${ }^{\circ} \mathrm{I}$

$\varepsilon \mathrm{I}^{\circ} \mathrm{I}$

$0 \tau^{\circ} \mathrm{I}$

Zt' I

st. I

9t' I

$t S^{\circ} \mathrm{I}$

$\angle S^{\circ} \mathrm{I}$

$09^{\circ} \mathrm{I}$

$59^{\circ} \mathrm{I}$

$99^{\circ} \mathrm{I}$

$88^{\circ}$ I

$\varepsilon 0^{\circ} z$

$\checkmark 0^{\circ} \mathrm{Z}$

$90 \%$

$\angle 0^{\circ} Z$

$80^{\circ} \tau$

$\varepsilon \mathrm{I} \cdot \tau$

$\varsigma \mathrm{I} \tau$

9I' $z$

$L I \cdot z$

$\angle I^{\prime} \mathrm{Z}$

$8 I^{\circ} \mathrm{r}$

$6 I^{\circ} Z$

I $Z \cdot z$

$\varsigma \tau \cdot \tau$

$9 z \cdot z$

$6 \tau \cdot \tau$

I $\varepsilon \cdot \tau$

$\varepsilon \varepsilon \varepsilon^{\prime}$

$\varepsilon \varepsilon \cdot \varsigma$

$\varepsilon \varepsilon \cdot \varsigma$

$\triangleright \varepsilon^{\circ} \varsigma$

$\varsigma \mathcal{S} \varsigma$

$\varsigma \mathcal{S} \varsigma$

$\varsigma \varepsilon^{\circ} \varsigma$

$9 \varepsilon \cdot S$

$9 \varepsilon \cdot \varsigma$

$\angle \varepsilon \cdot S$

$6 \varepsilon \varsigma$

$0 t^{\circ} \varsigma$

It $S$

It $\subseteq$ -

$6 L^{\circ} \mathrm{S}$

$28^{\circ} \mathrm{s}$

$20^{\circ} \mathrm{L}$

$70^{\circ} \mathrm{L}$

ยเวดว $92^{\circ} L$

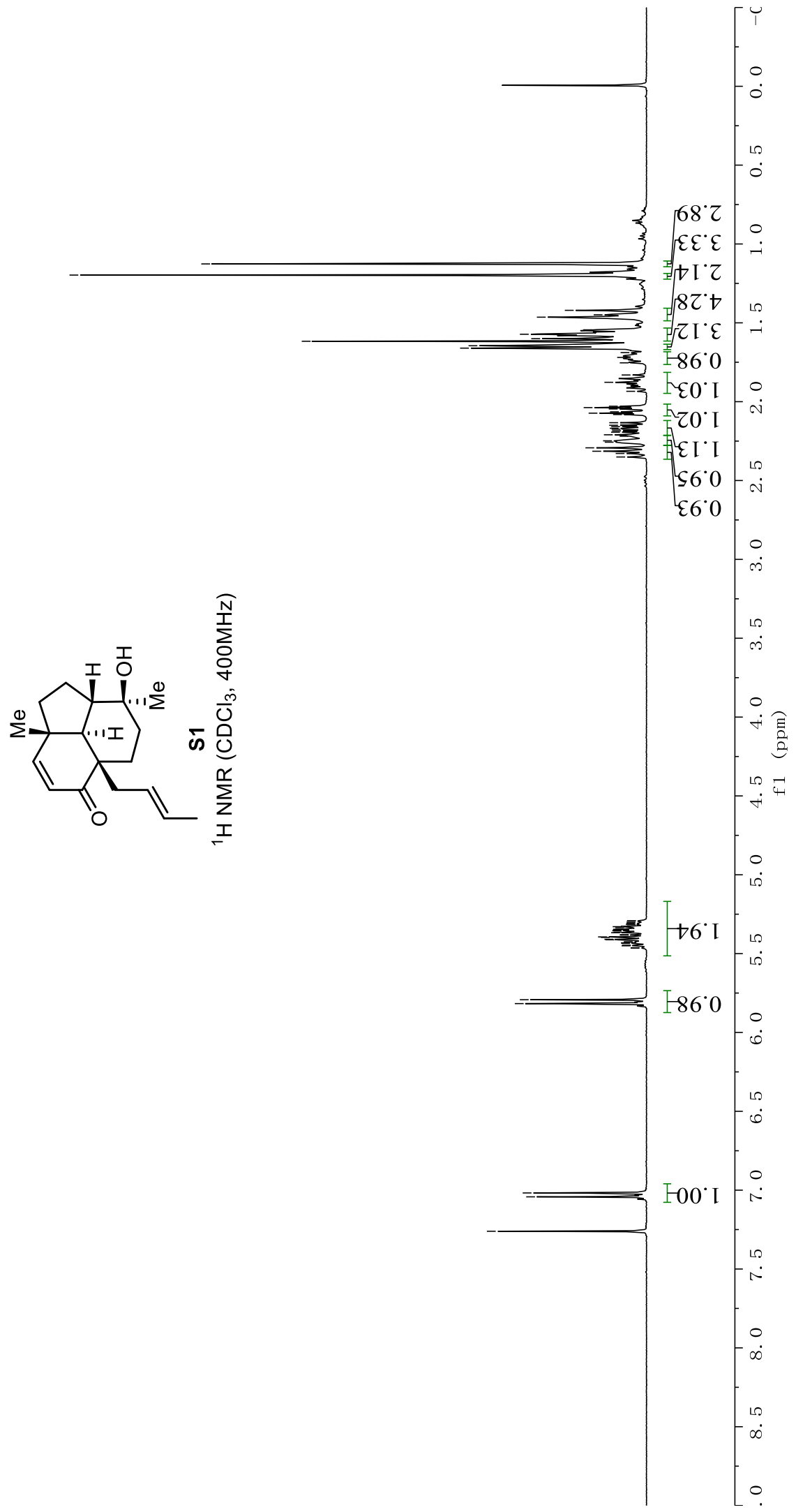




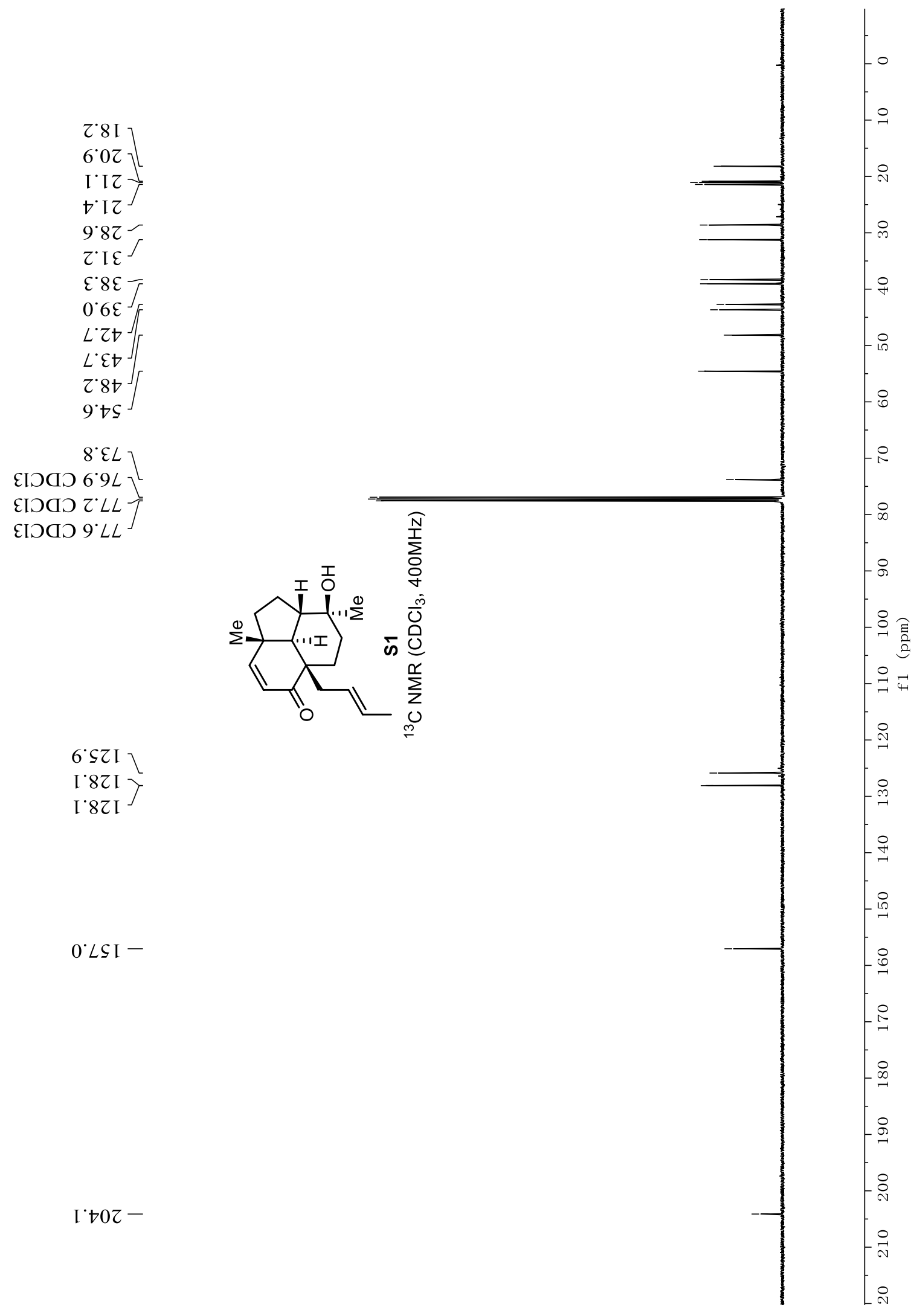




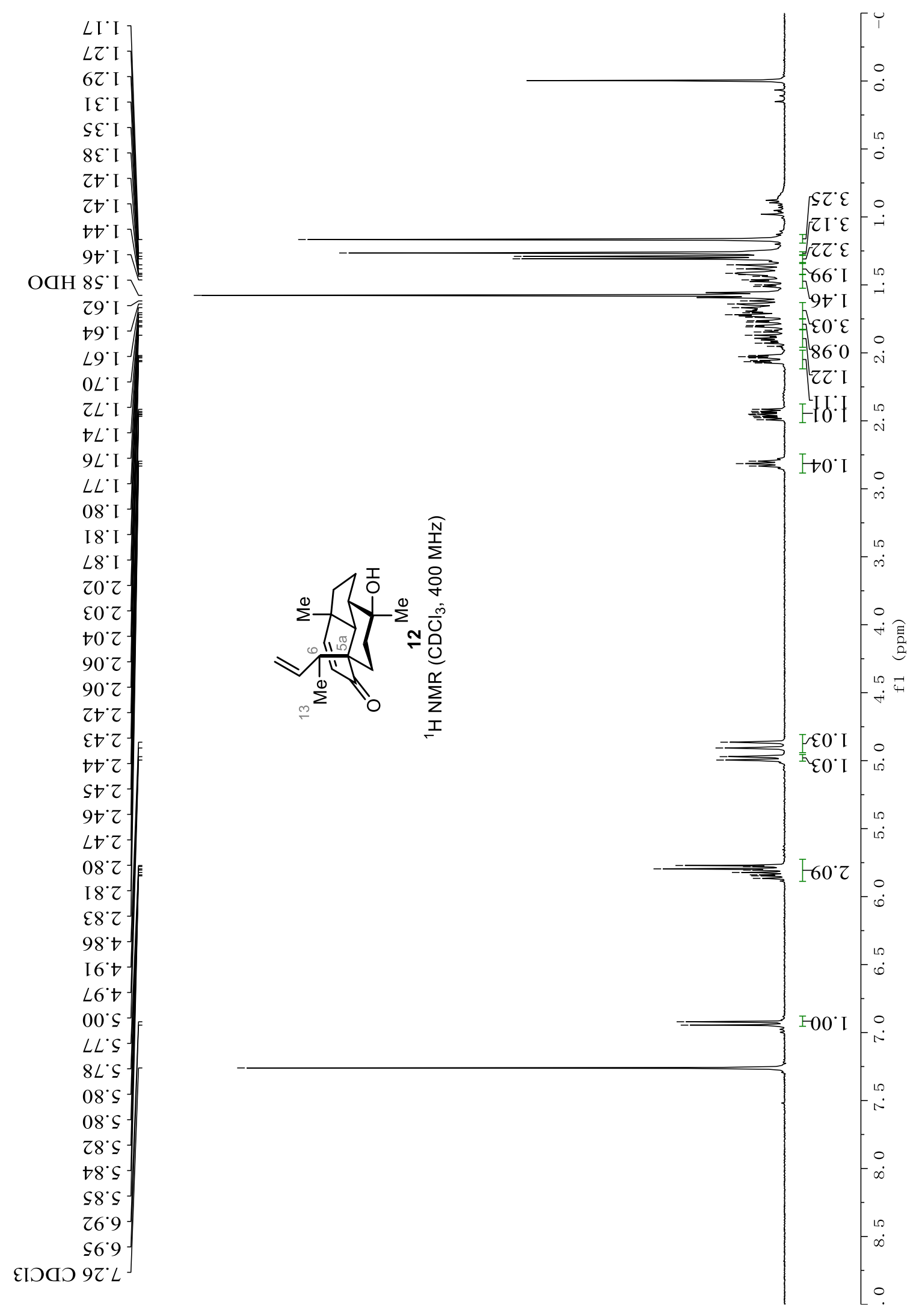




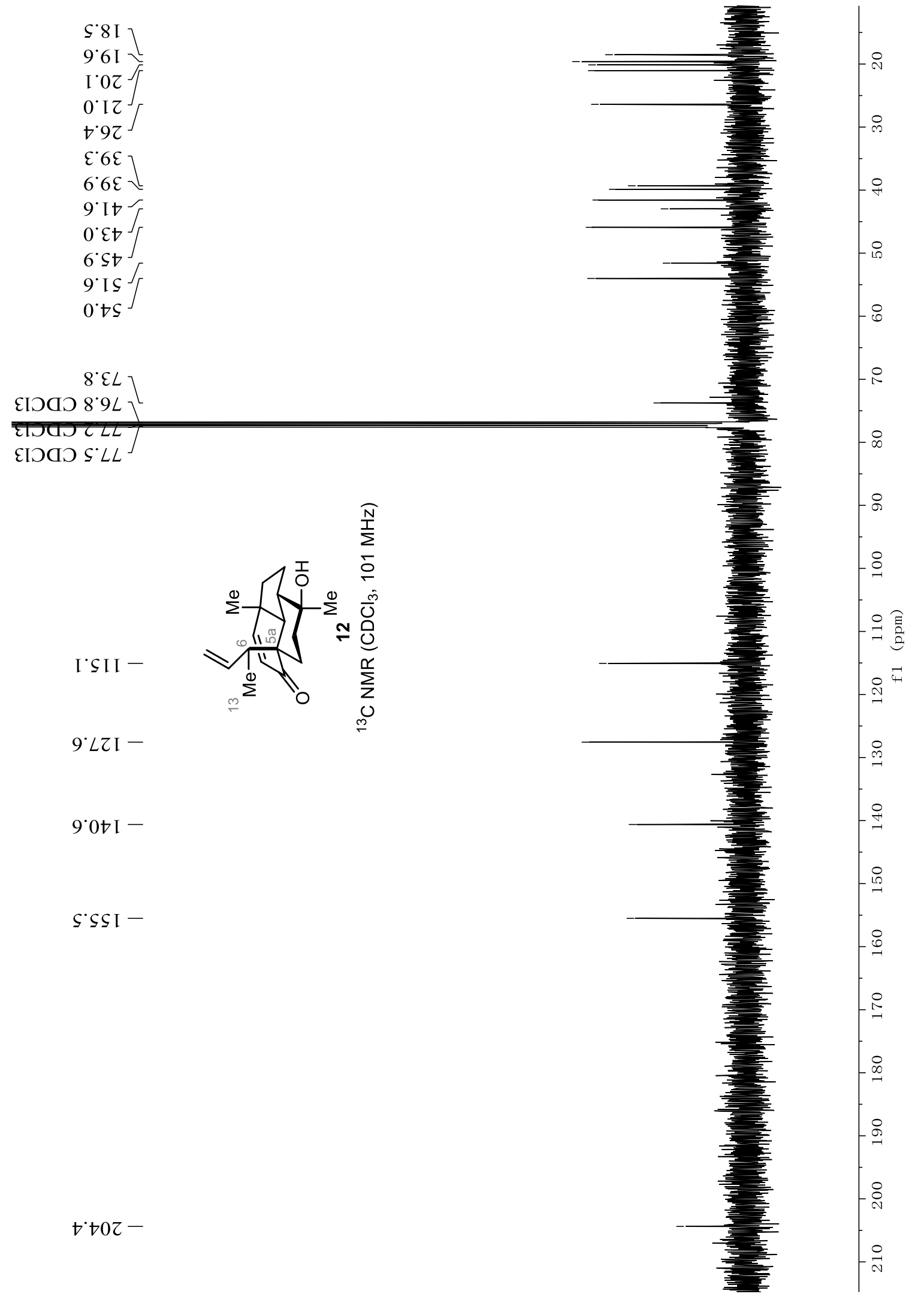




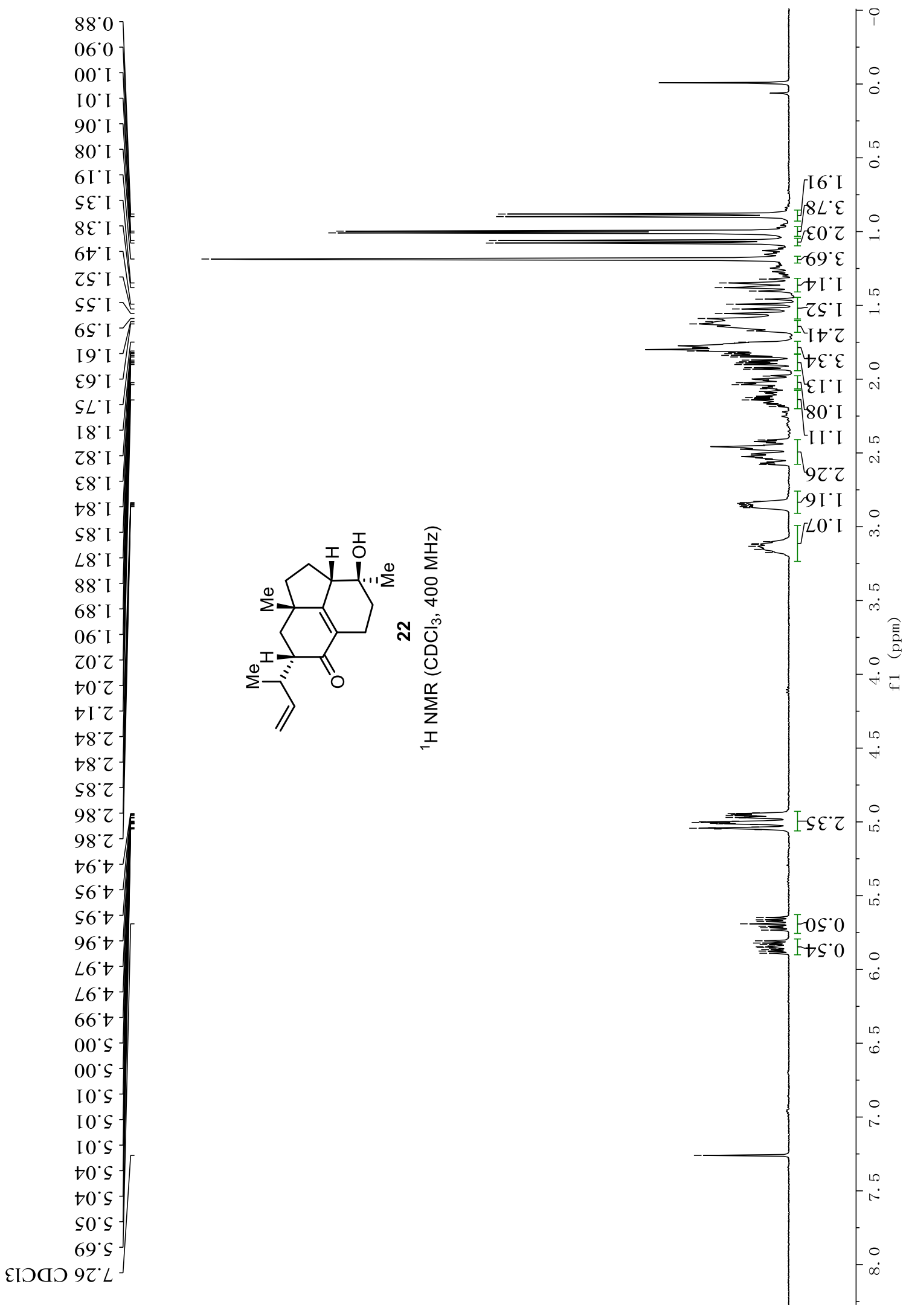




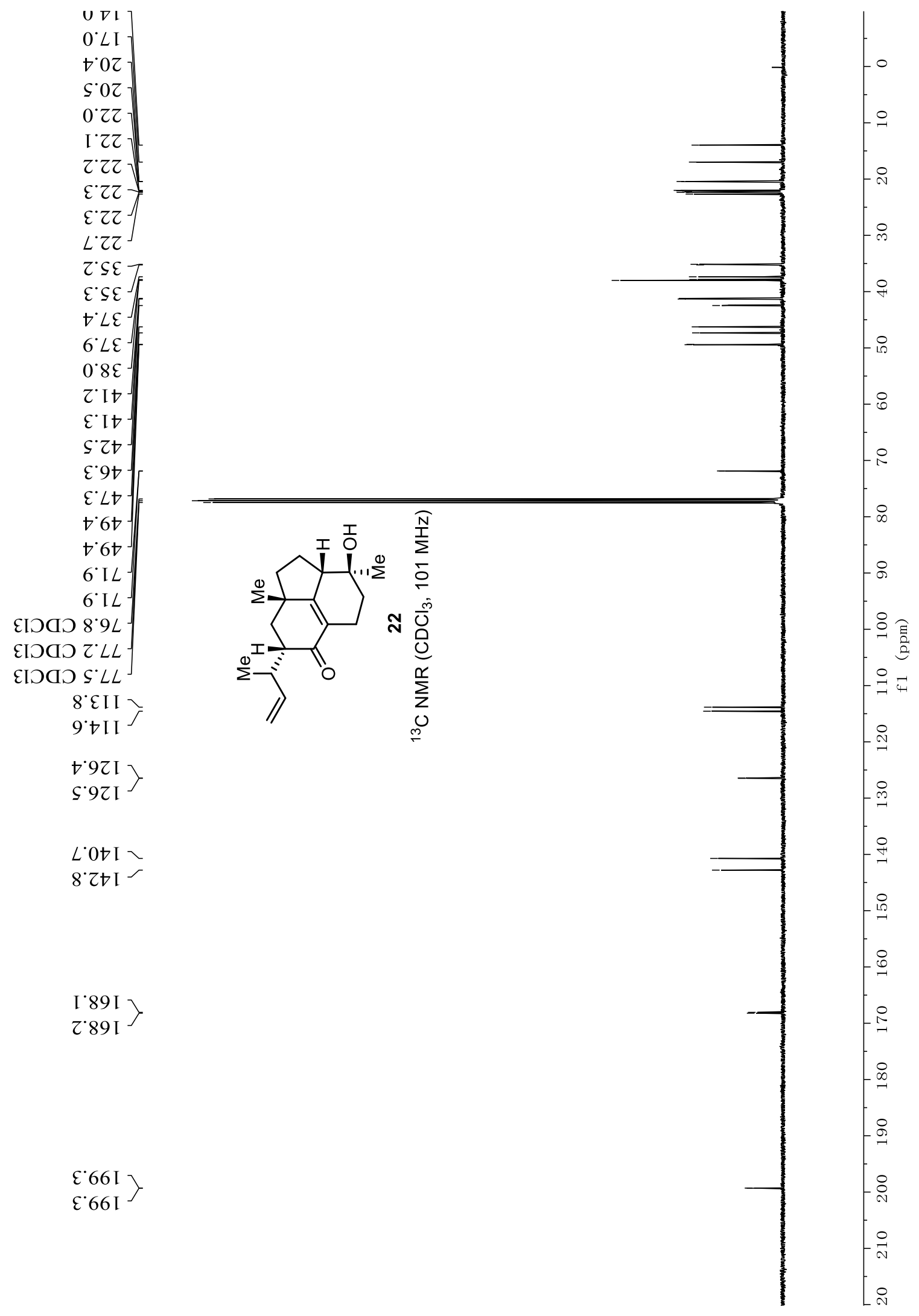




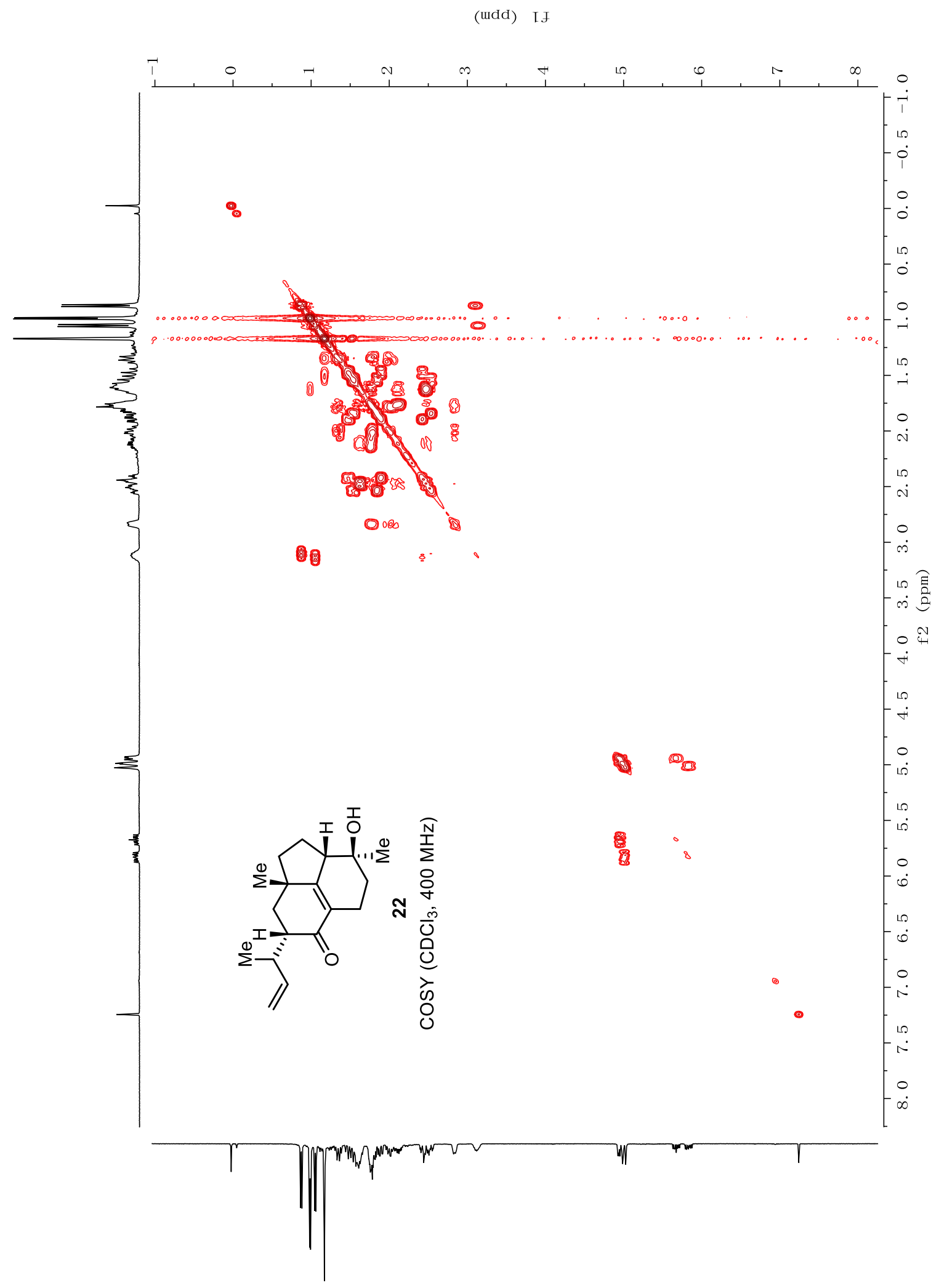




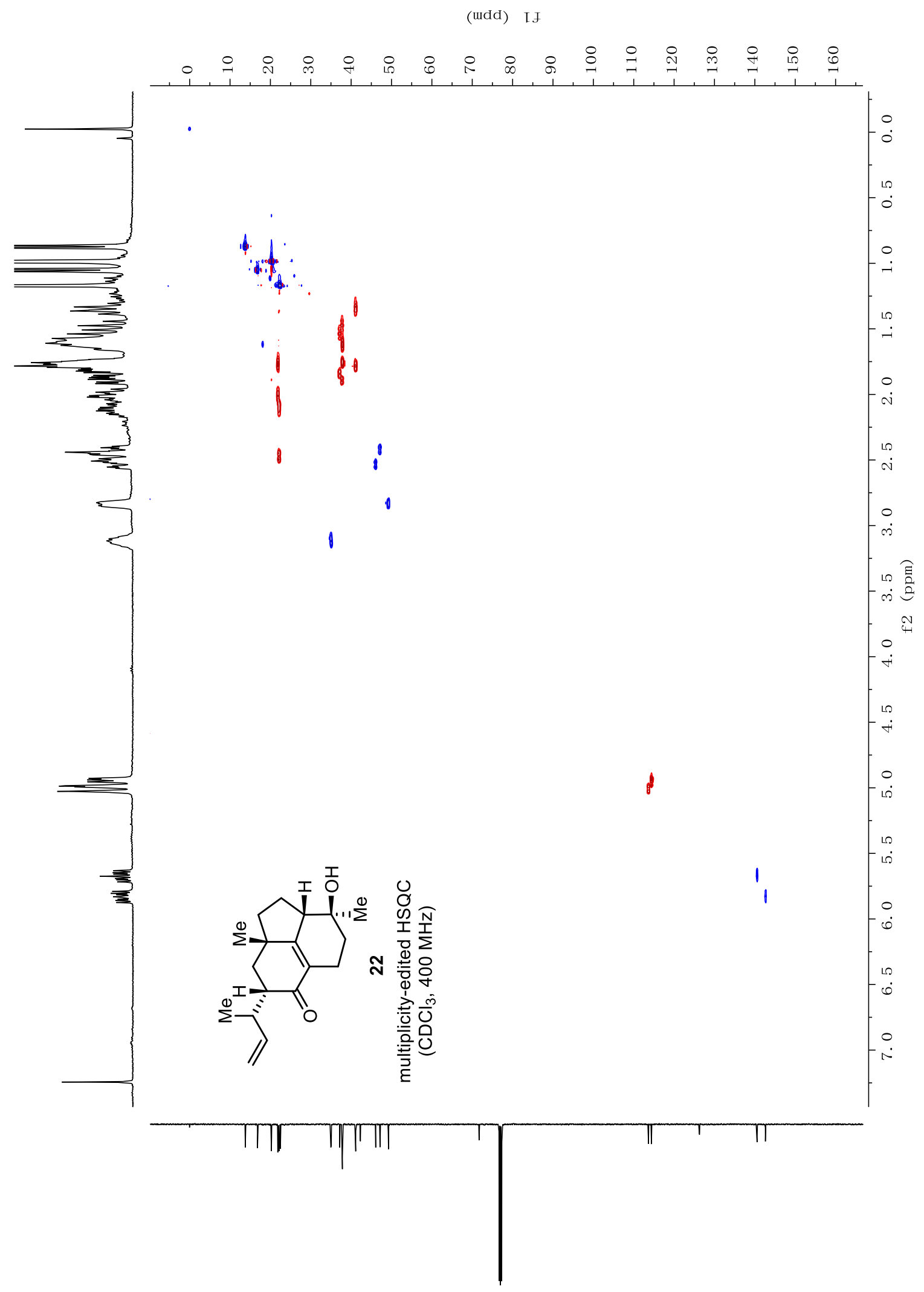




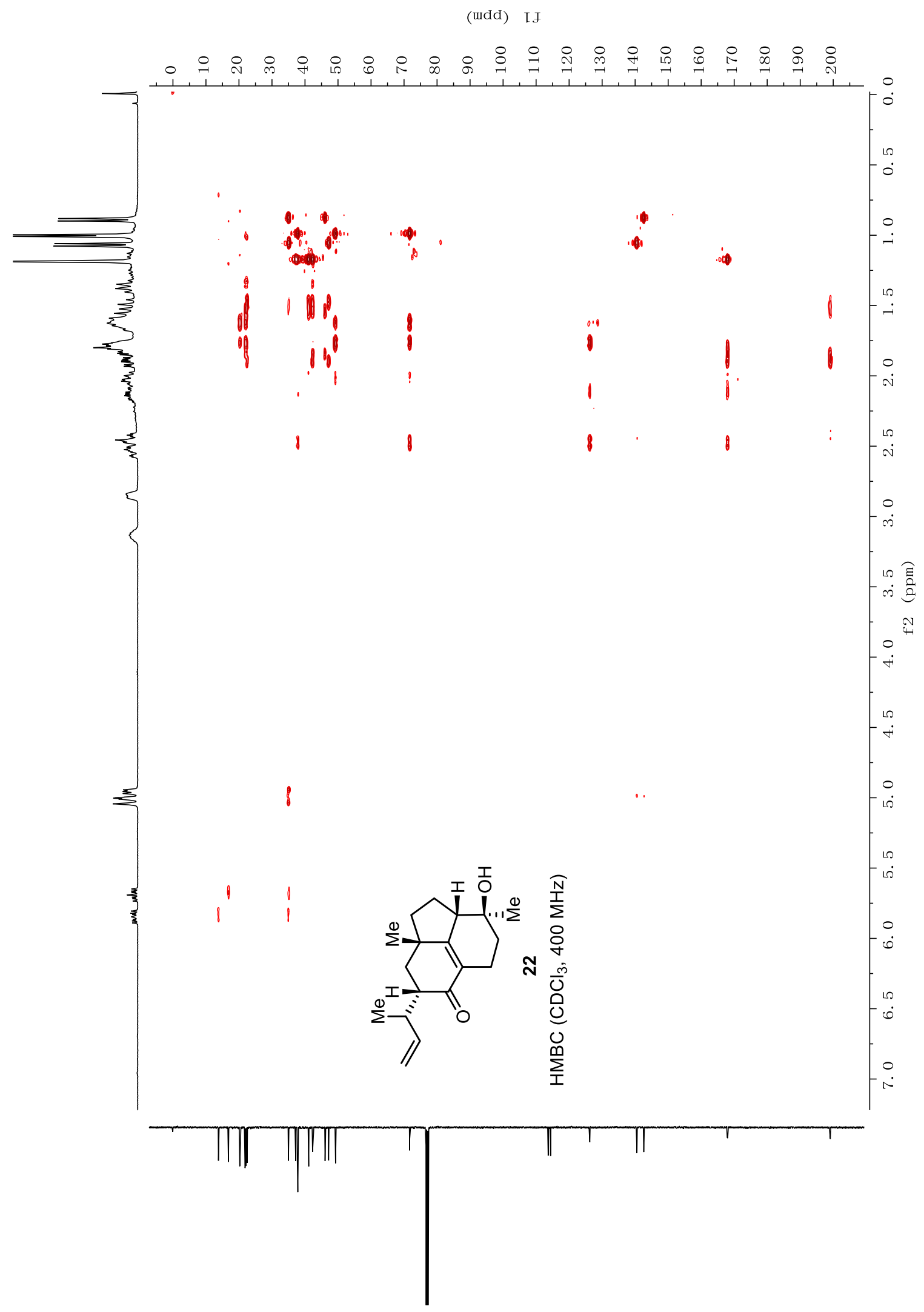




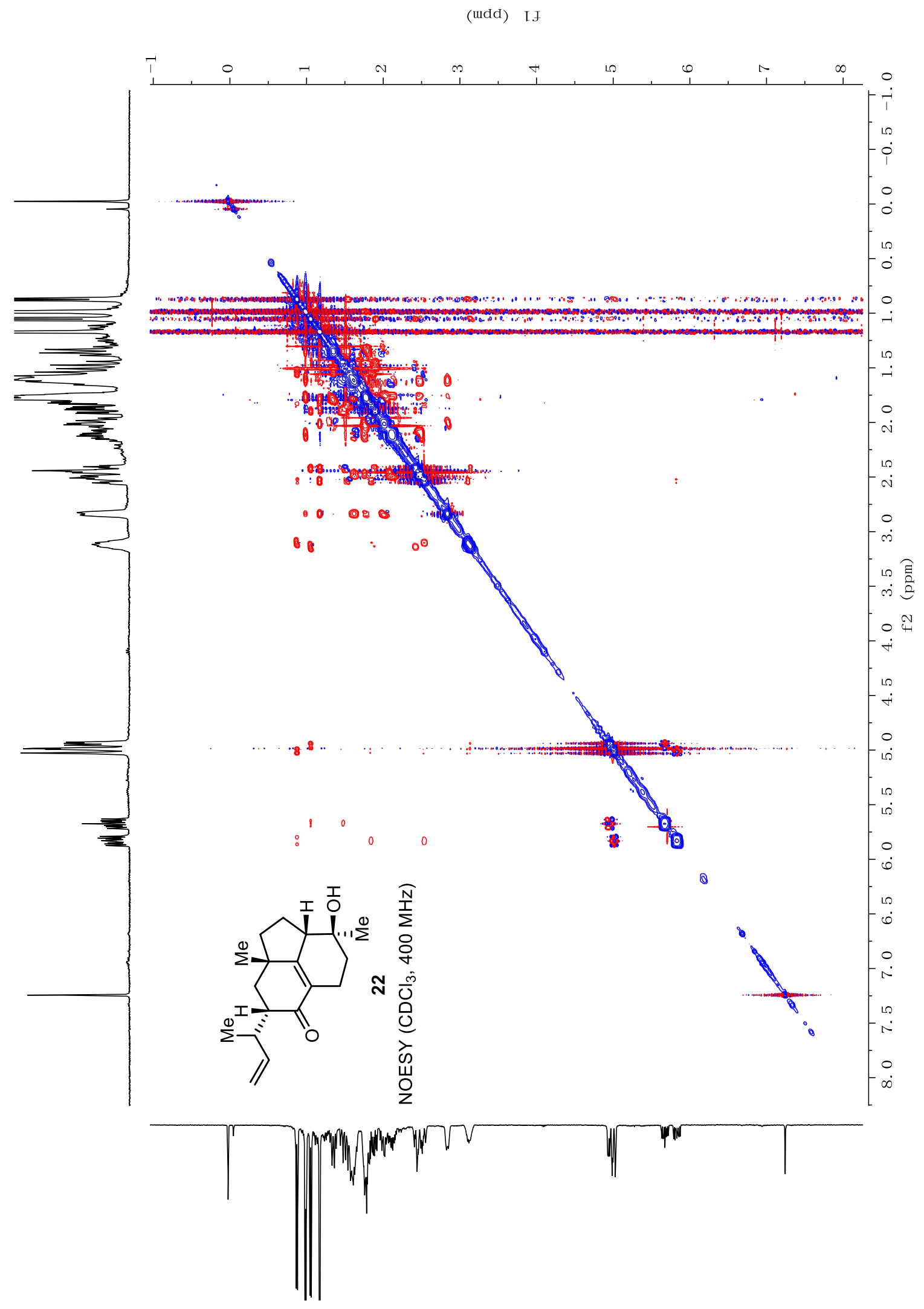




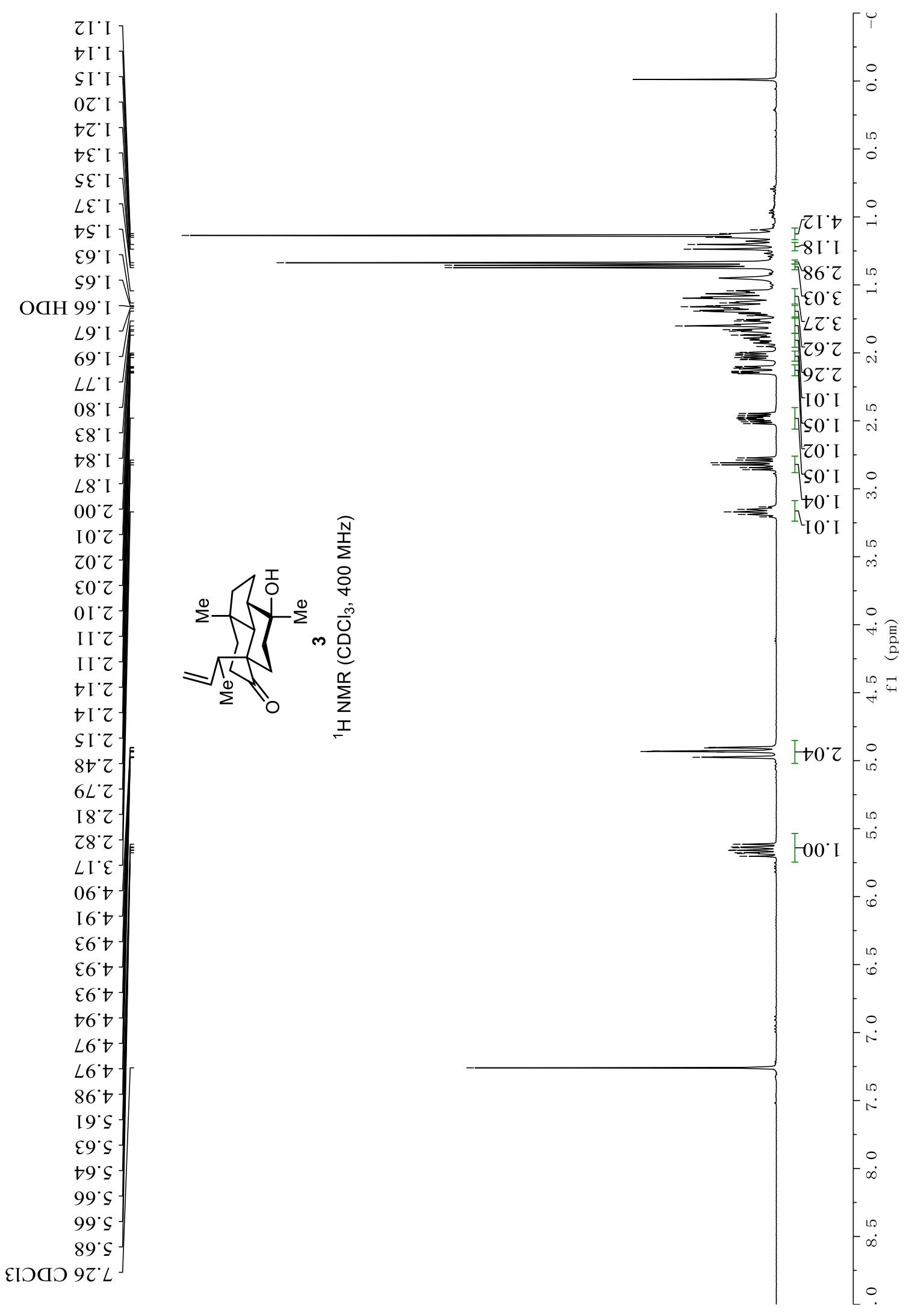




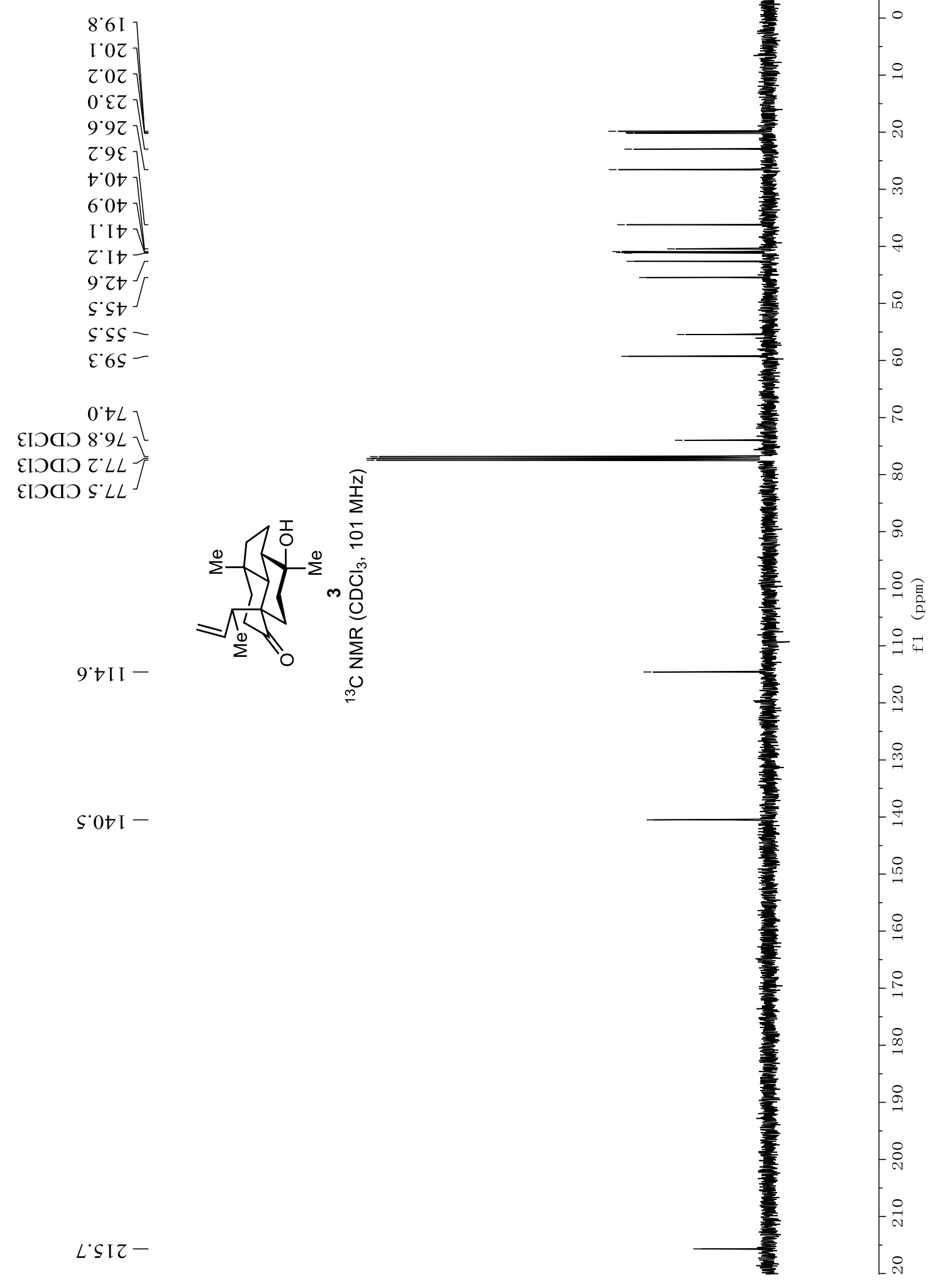




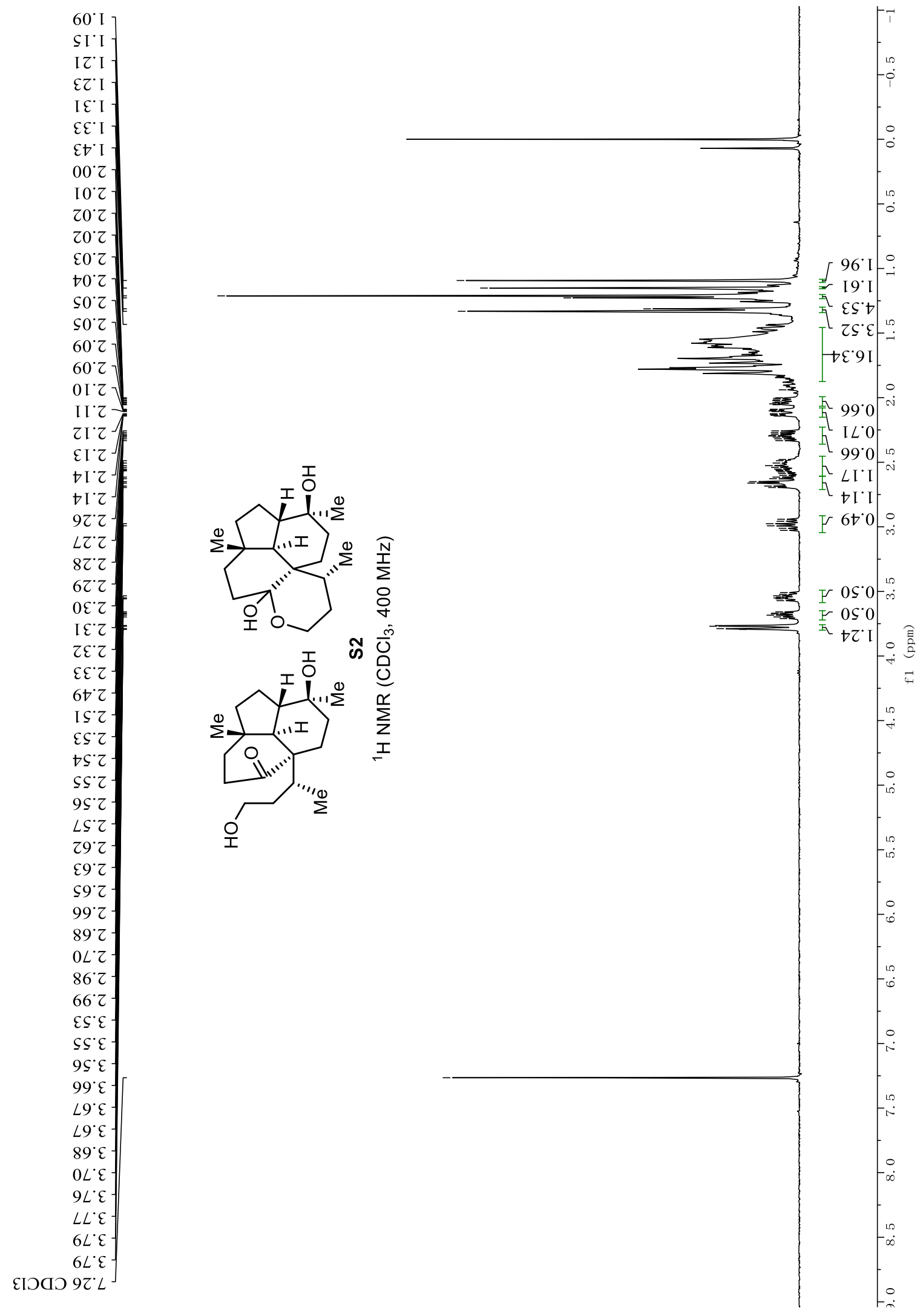




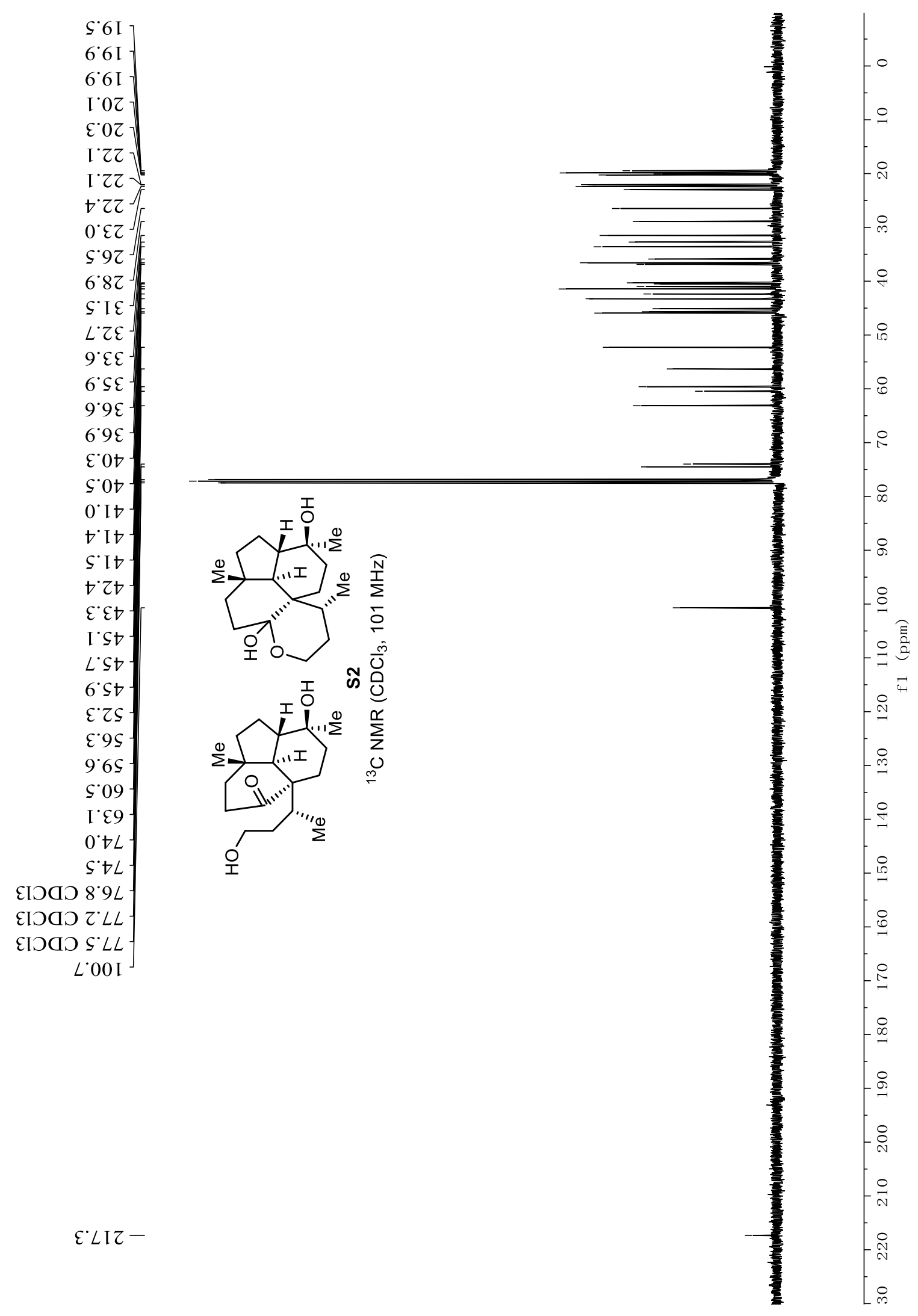




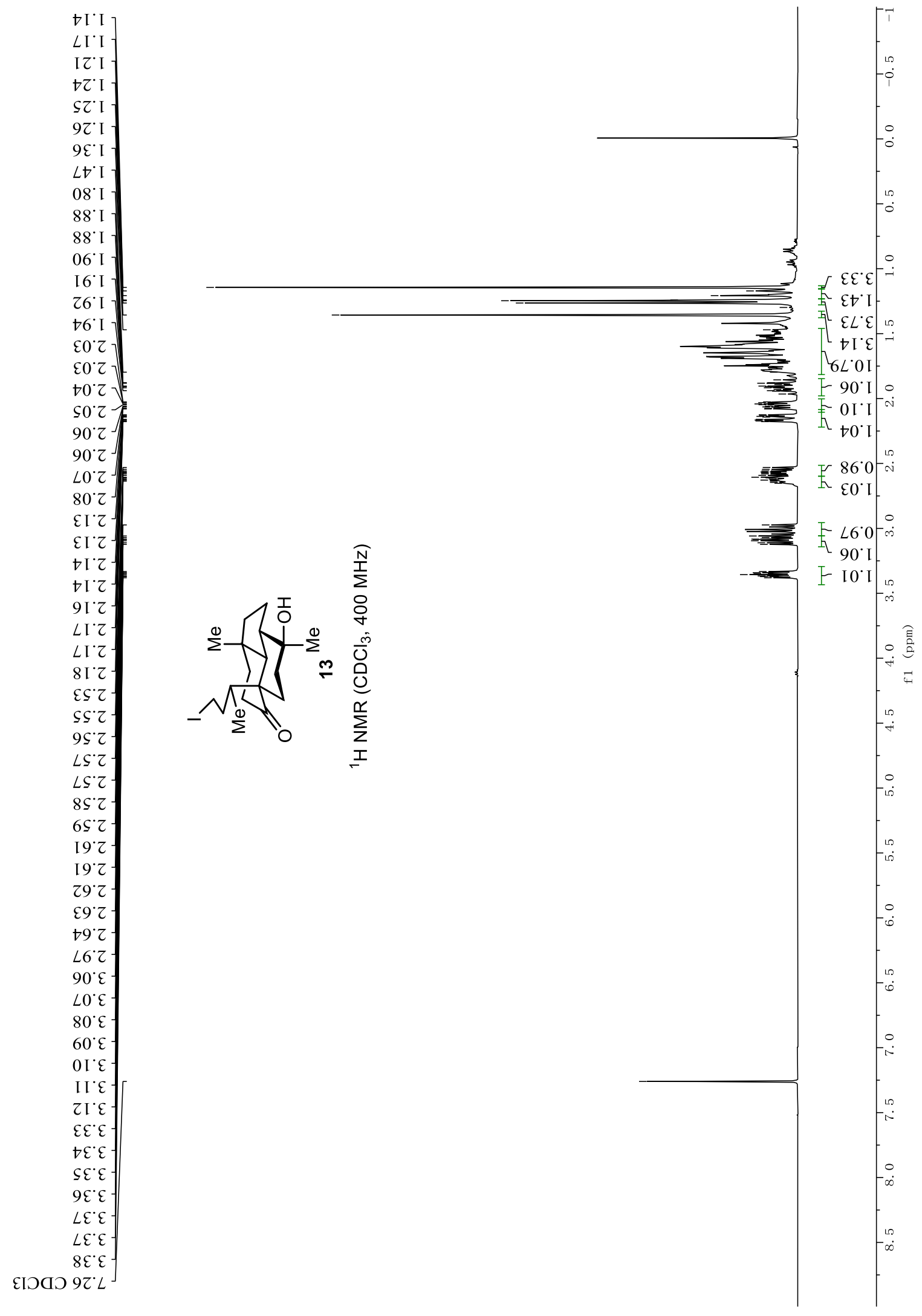




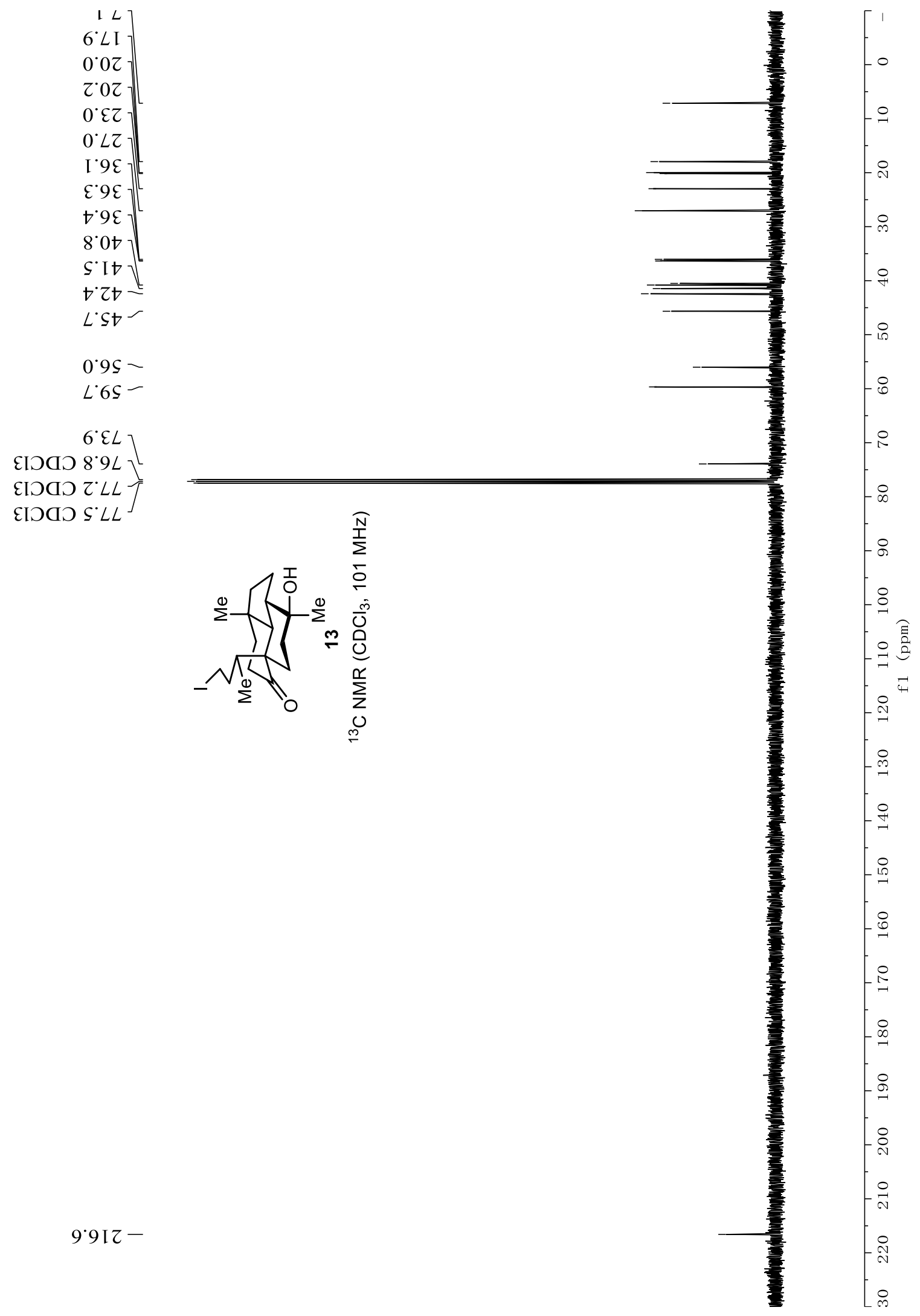




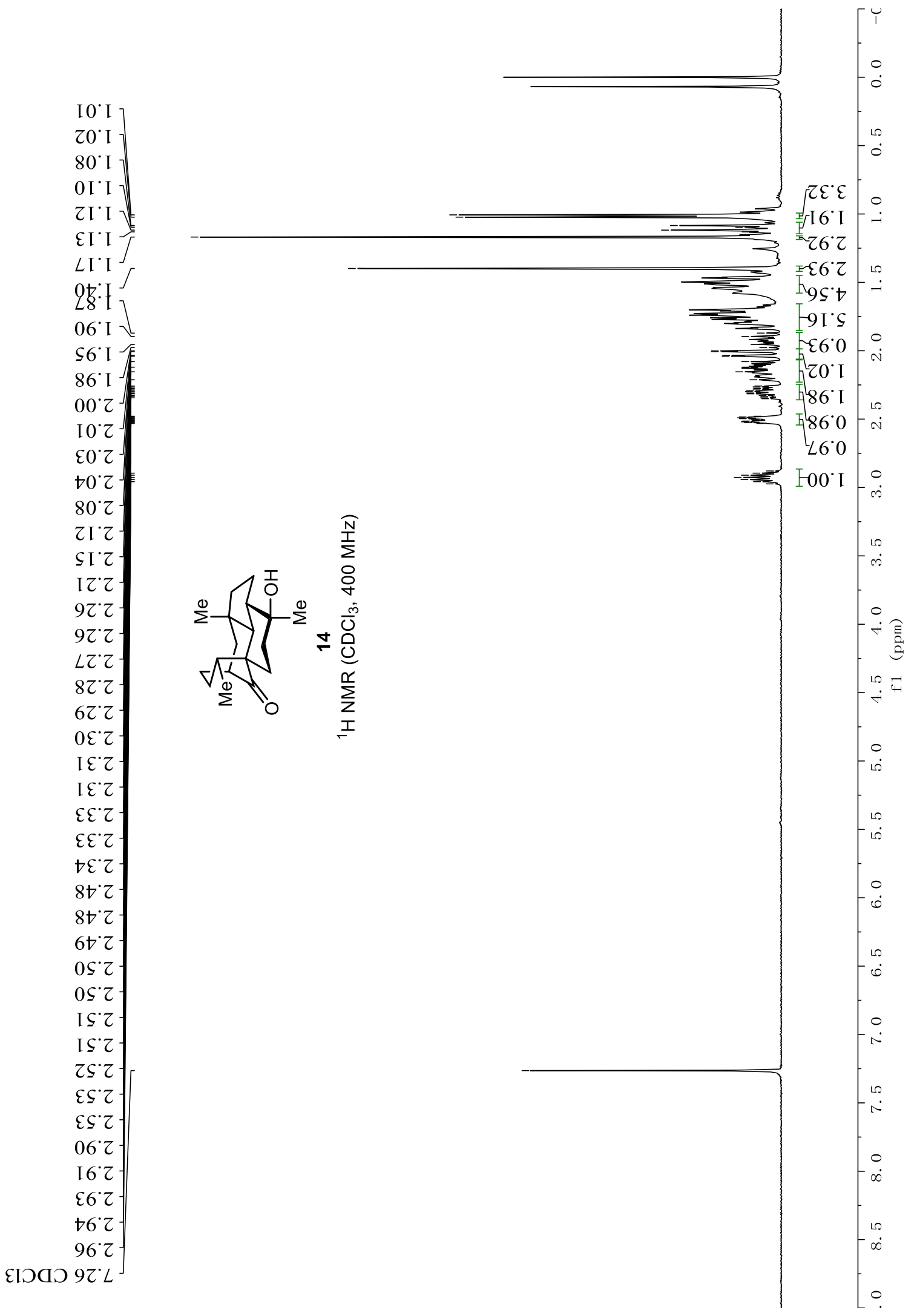



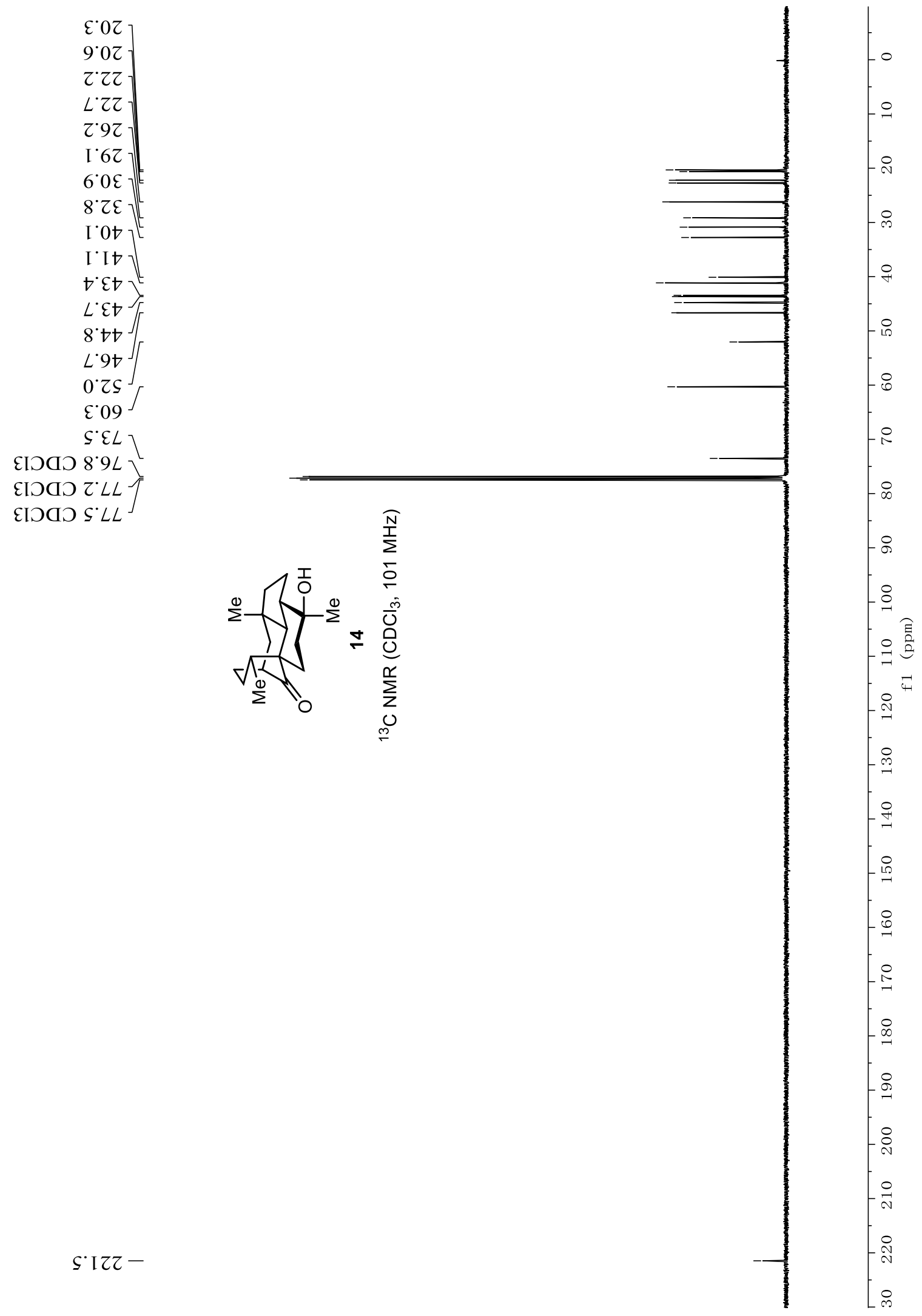


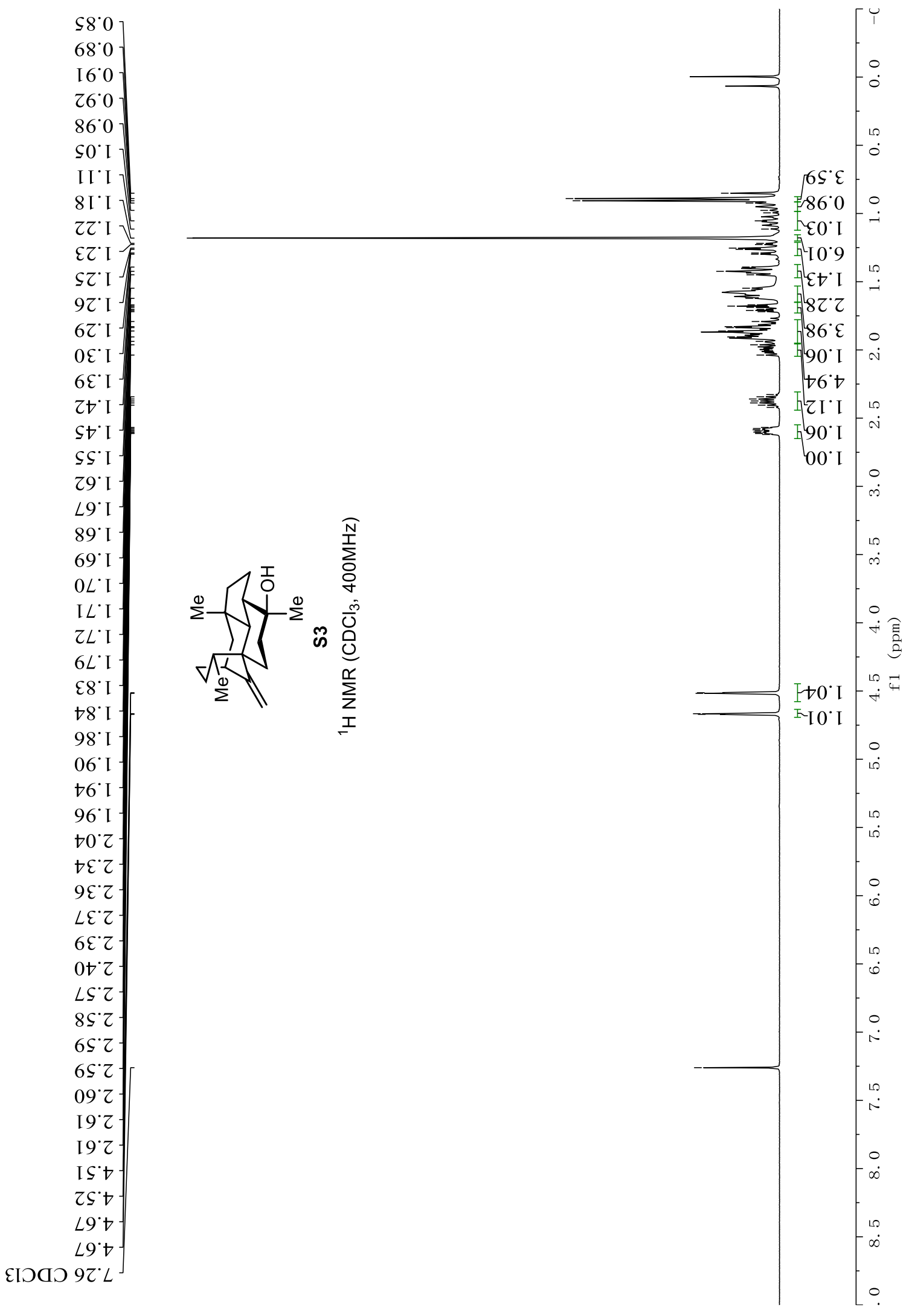




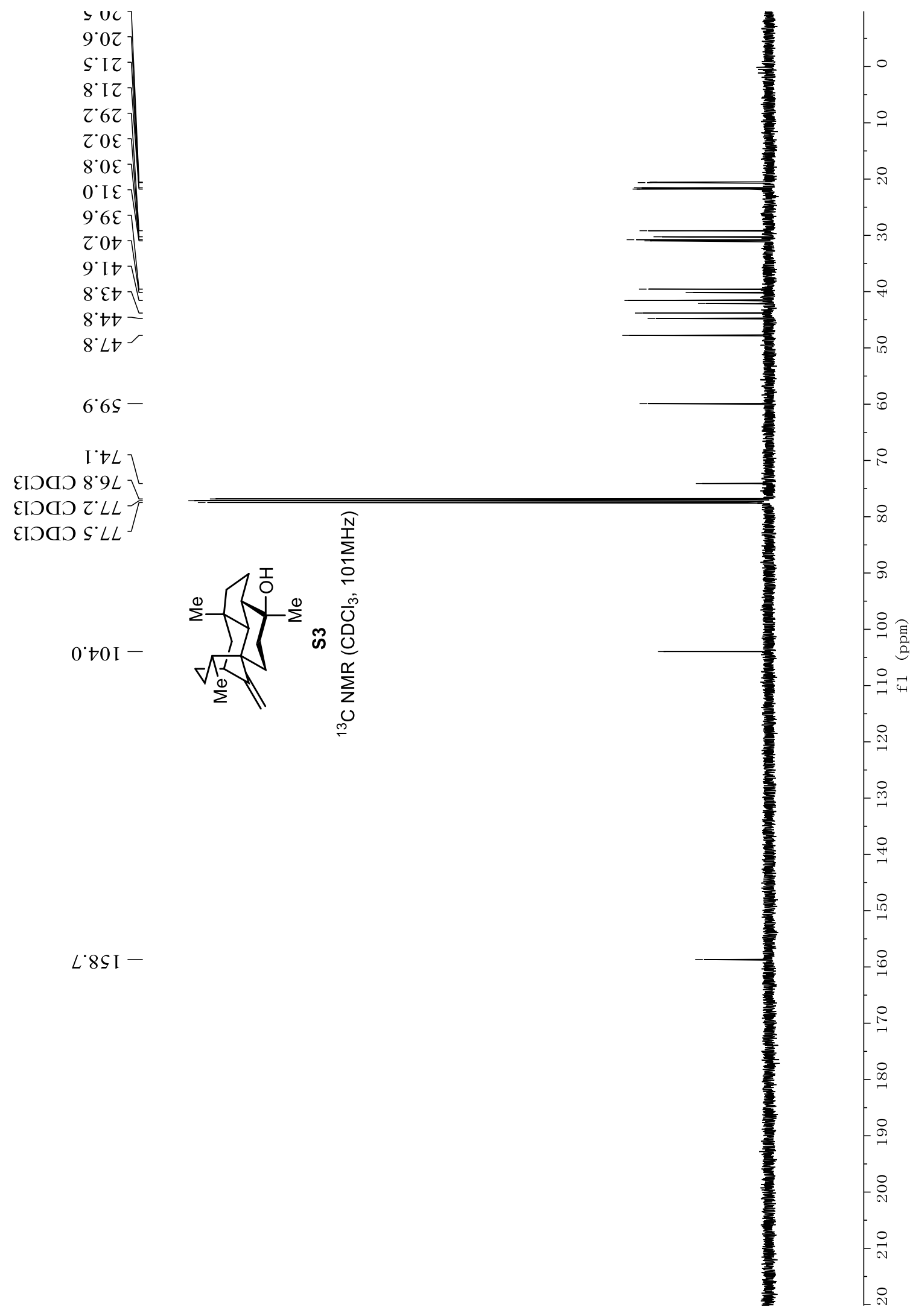




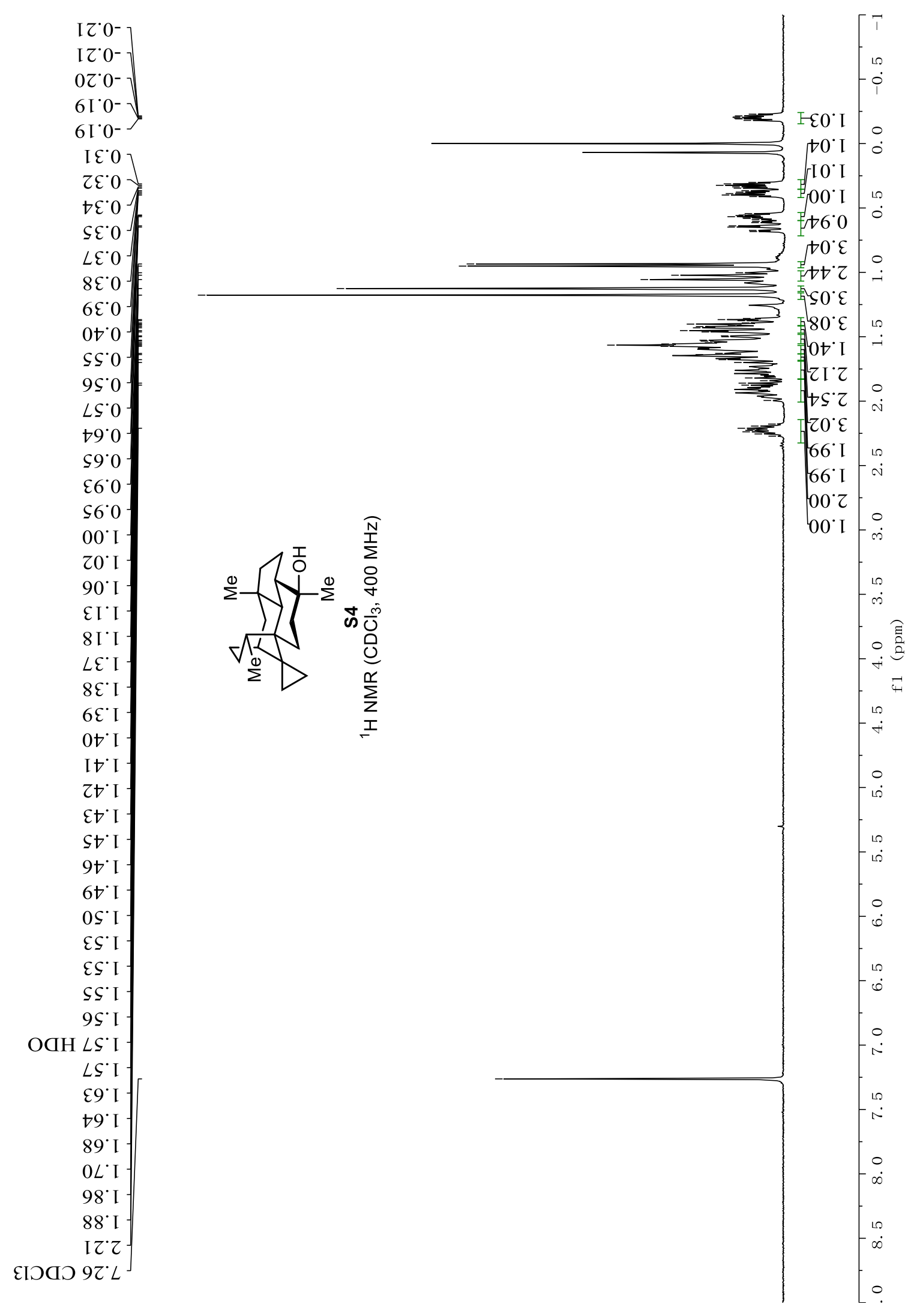




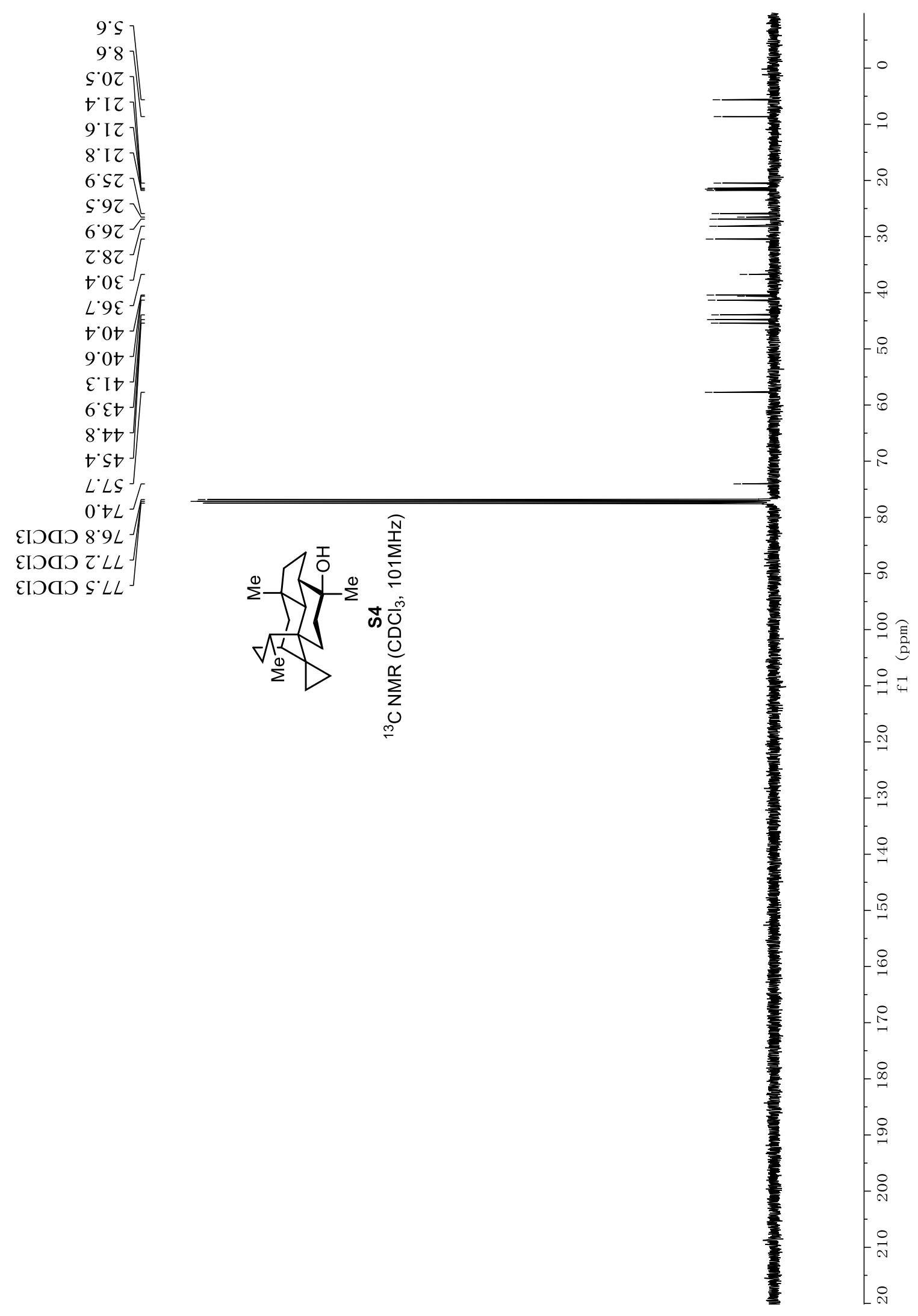




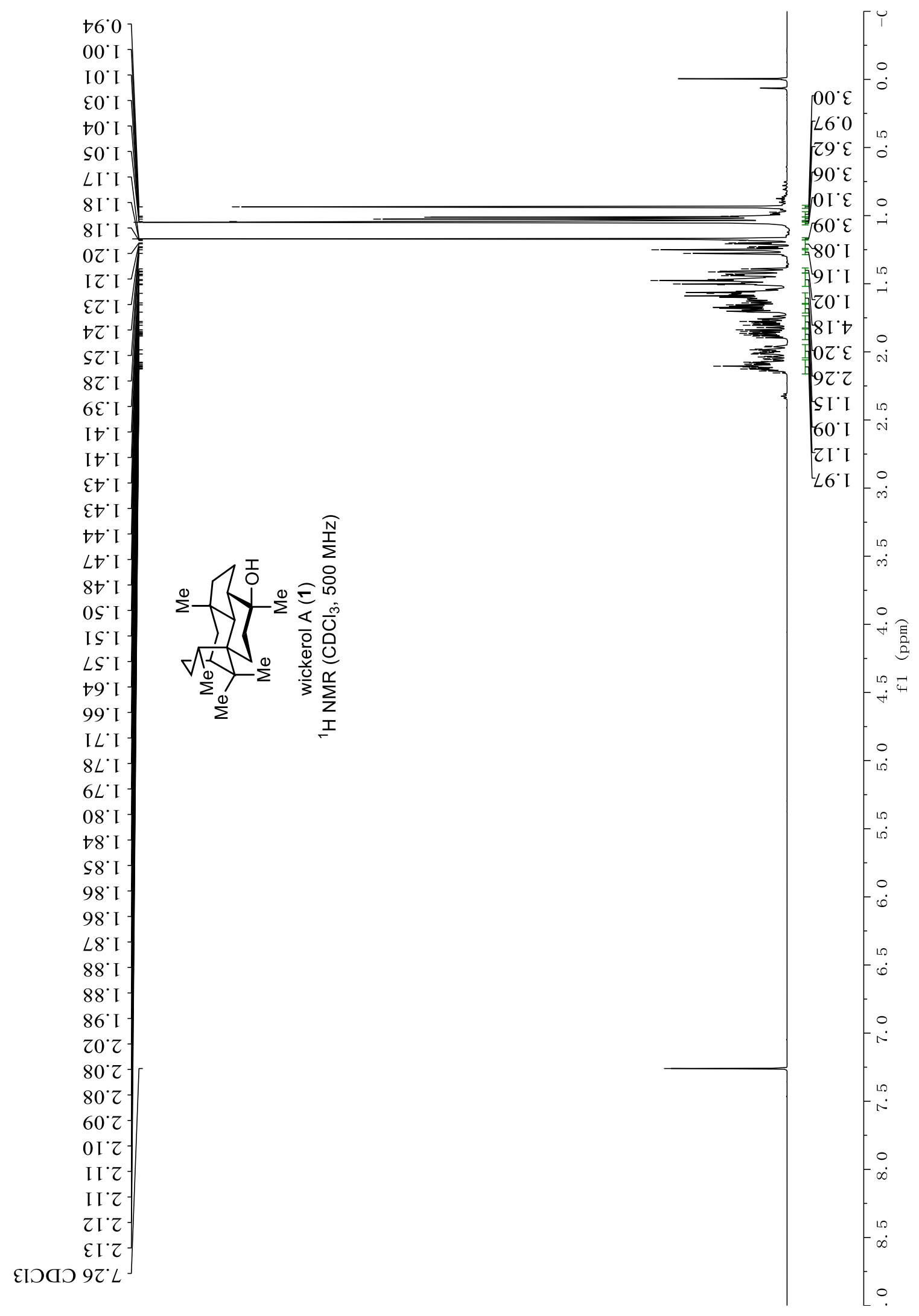




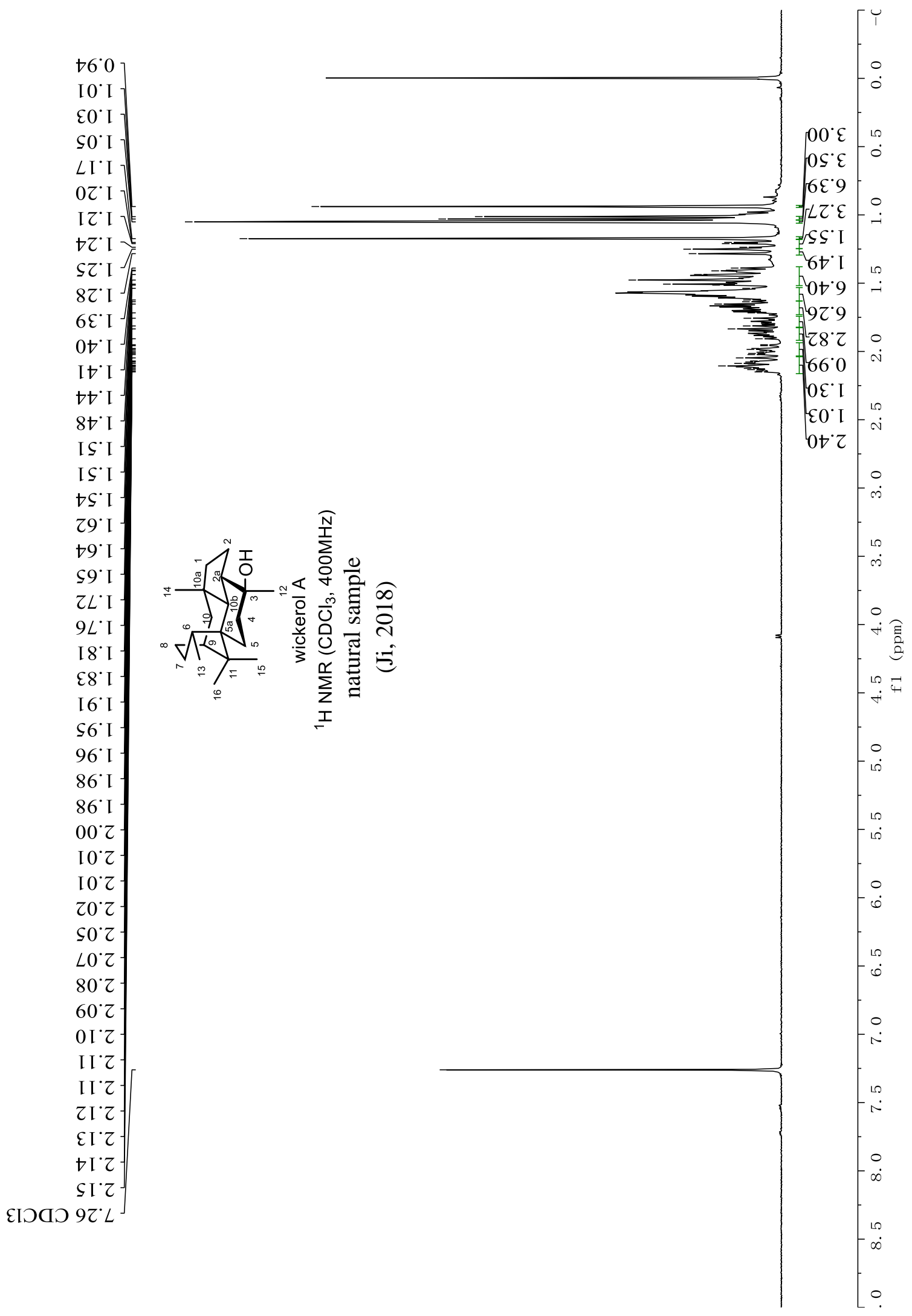




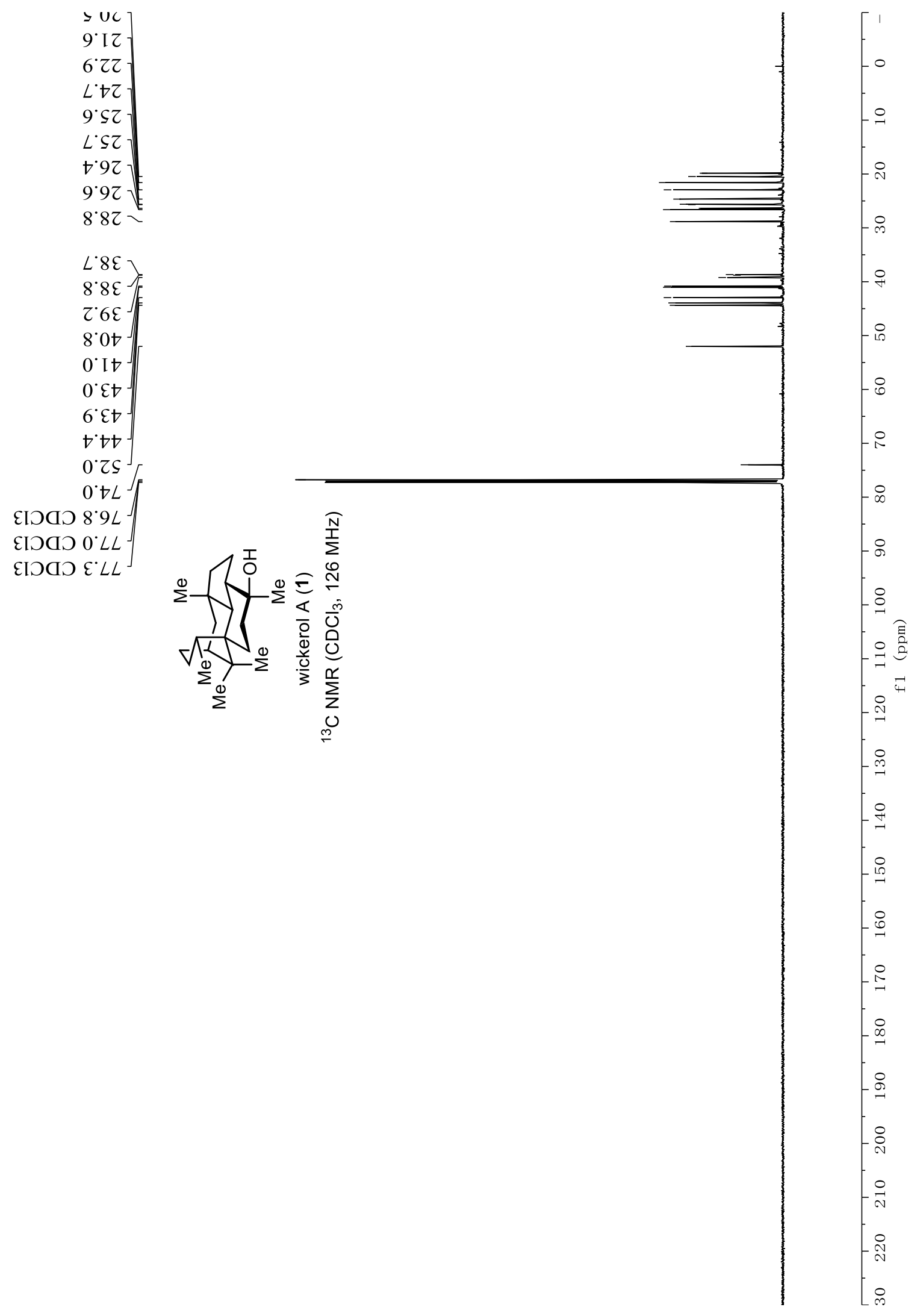




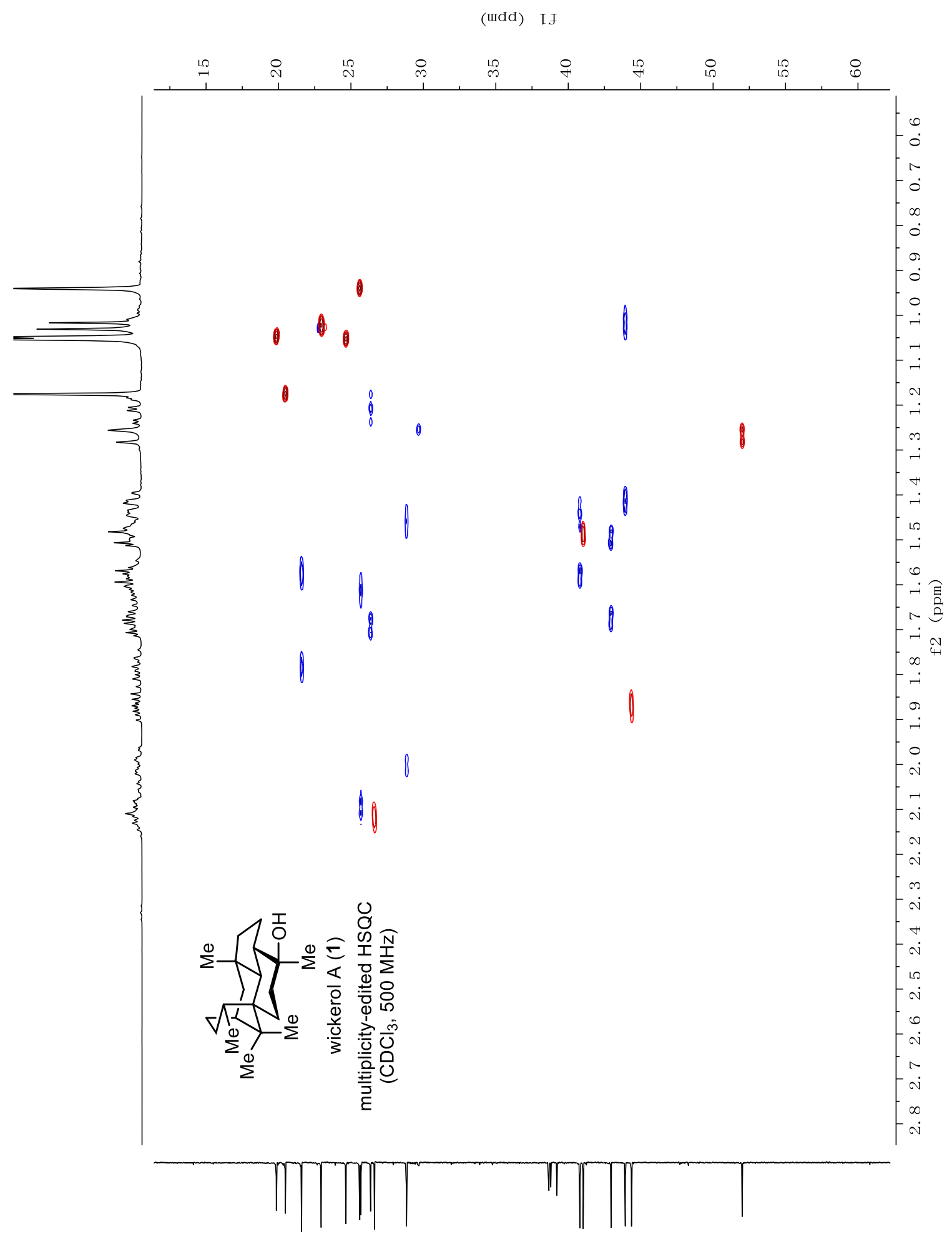




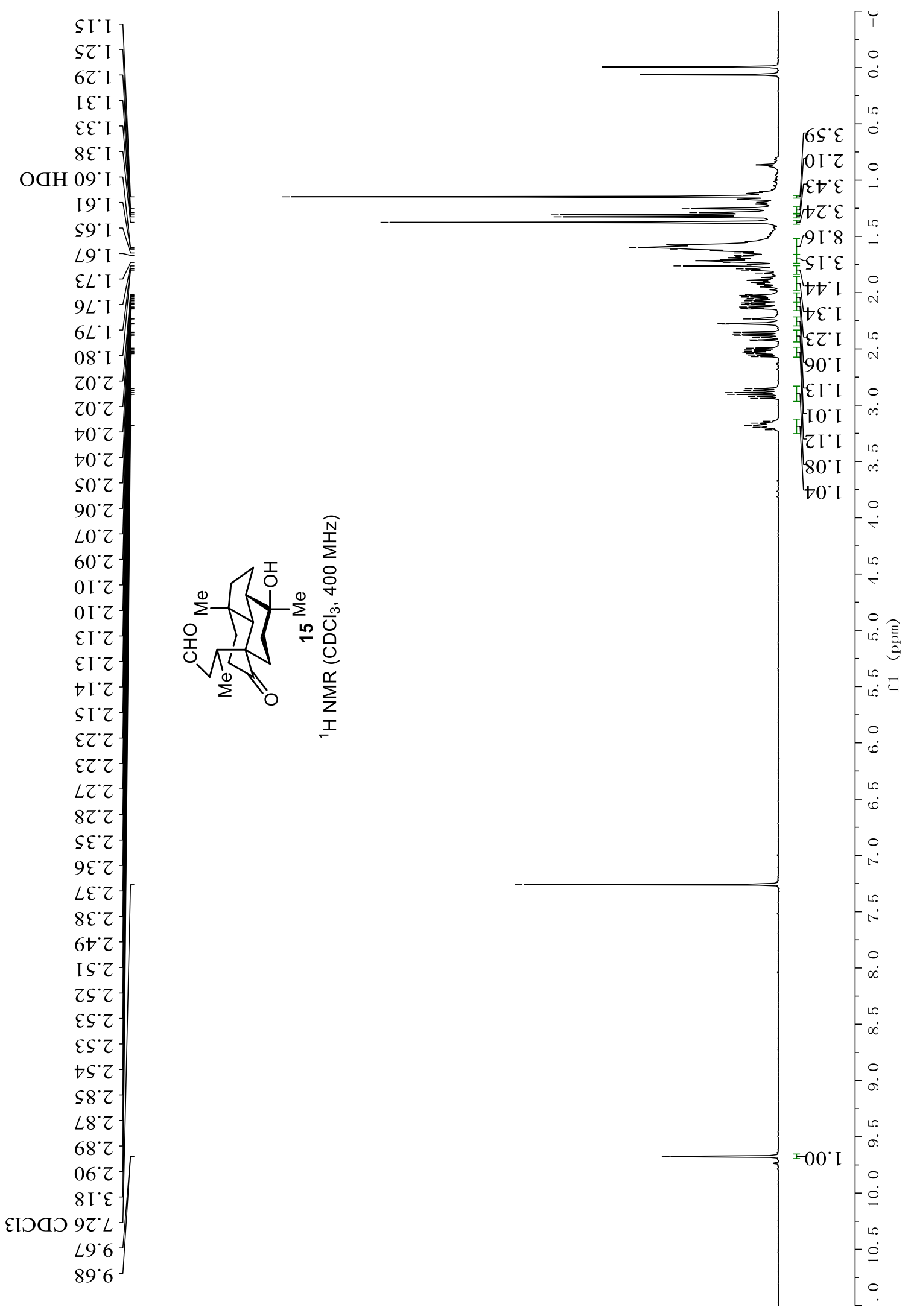




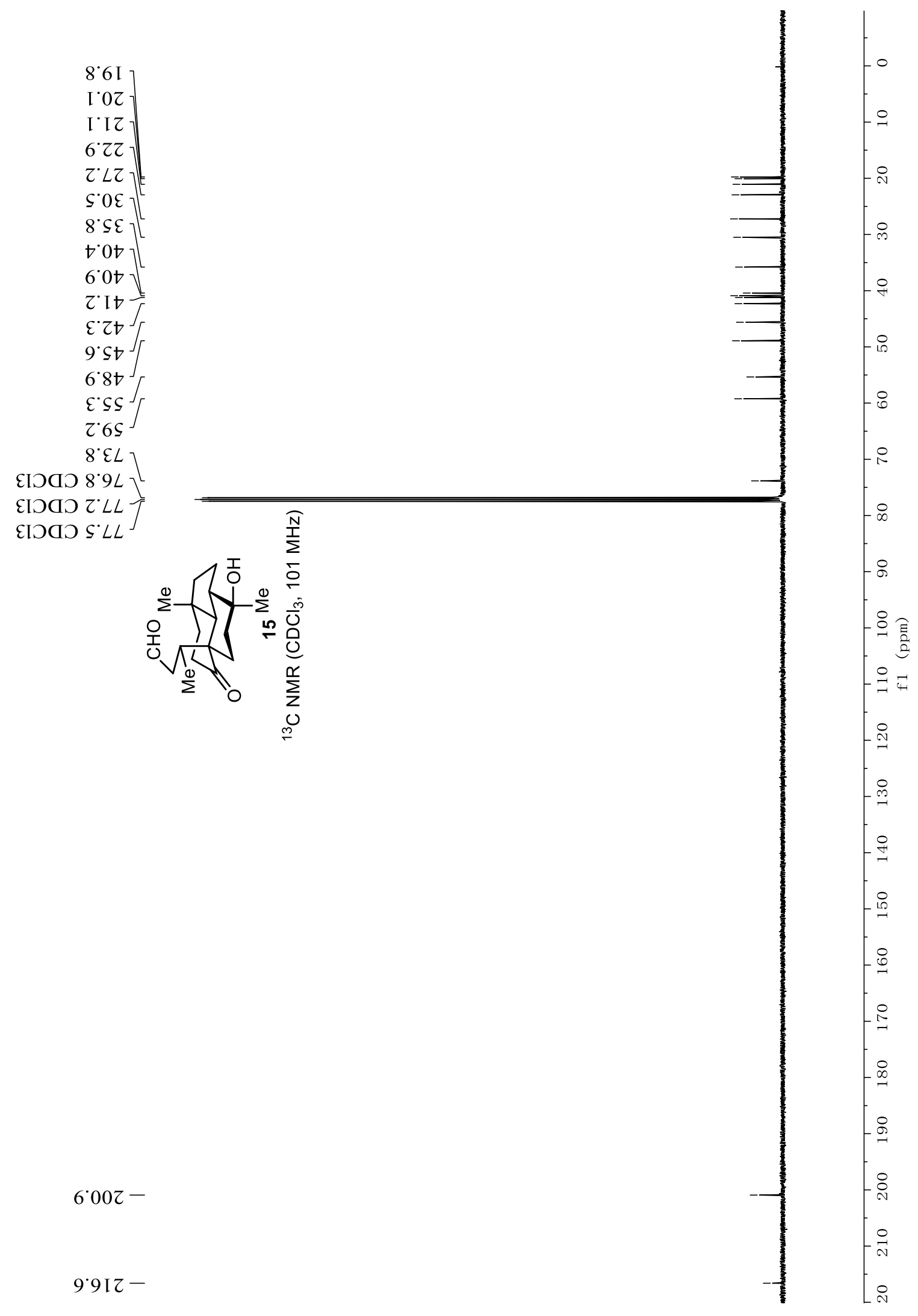




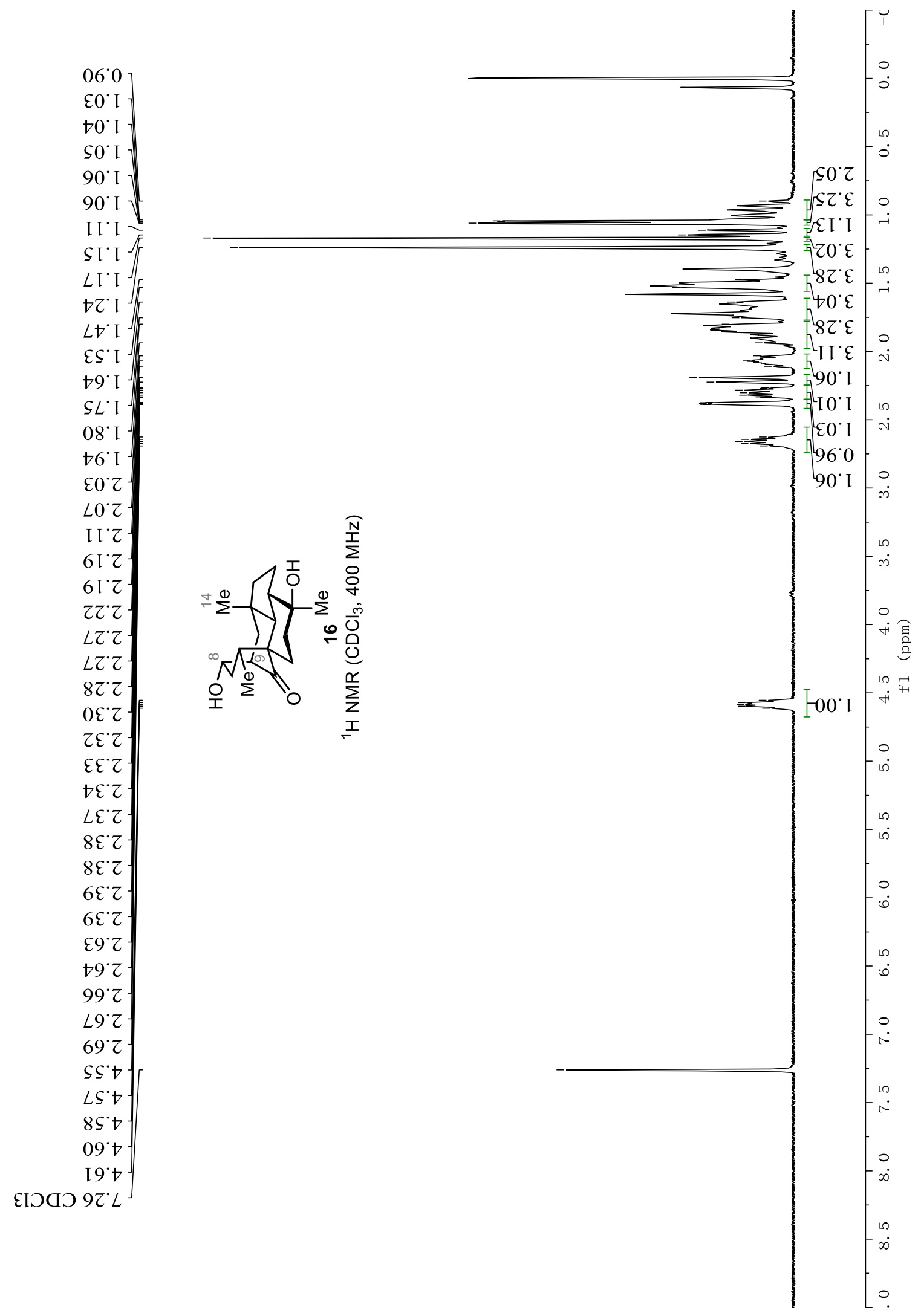




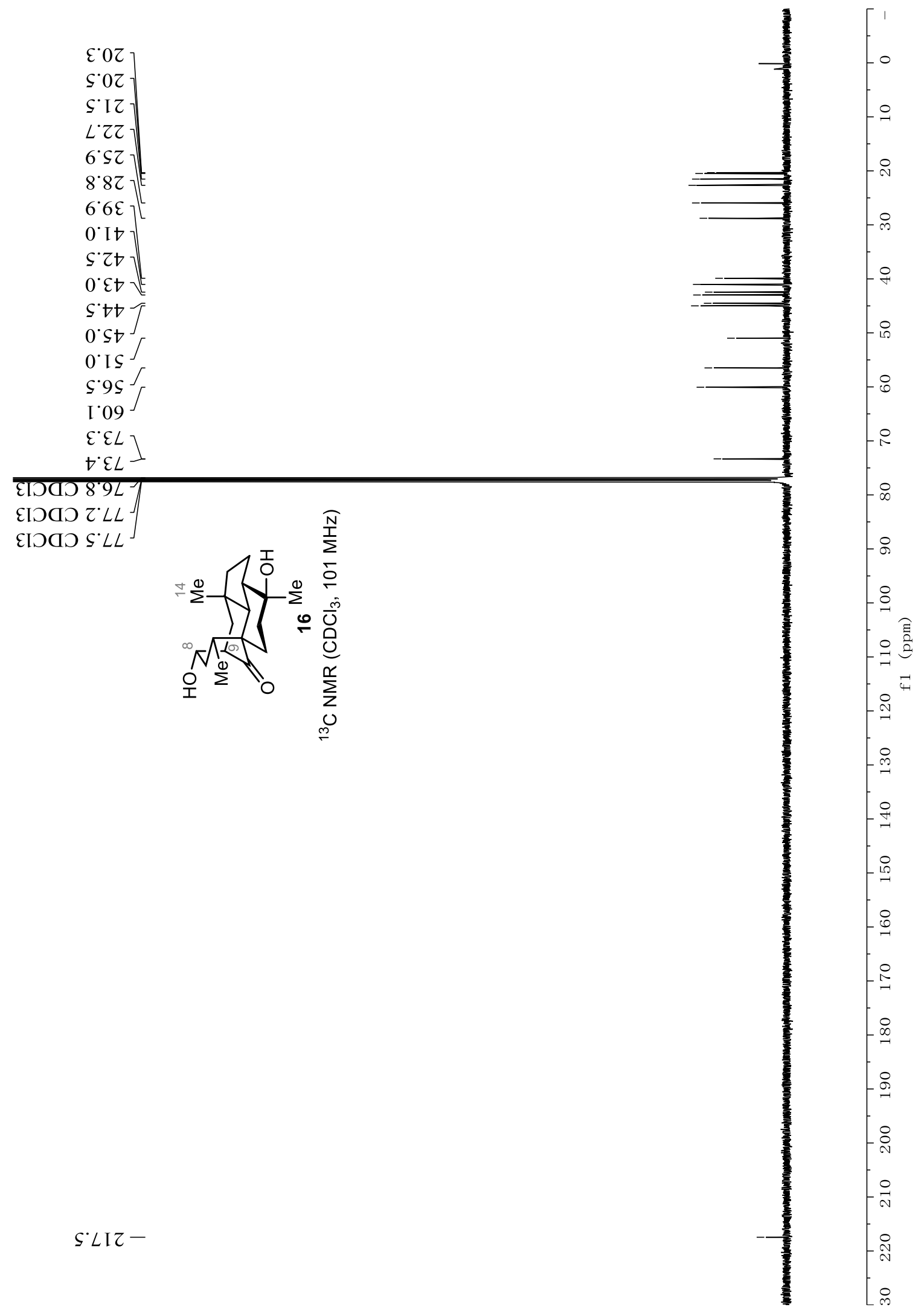




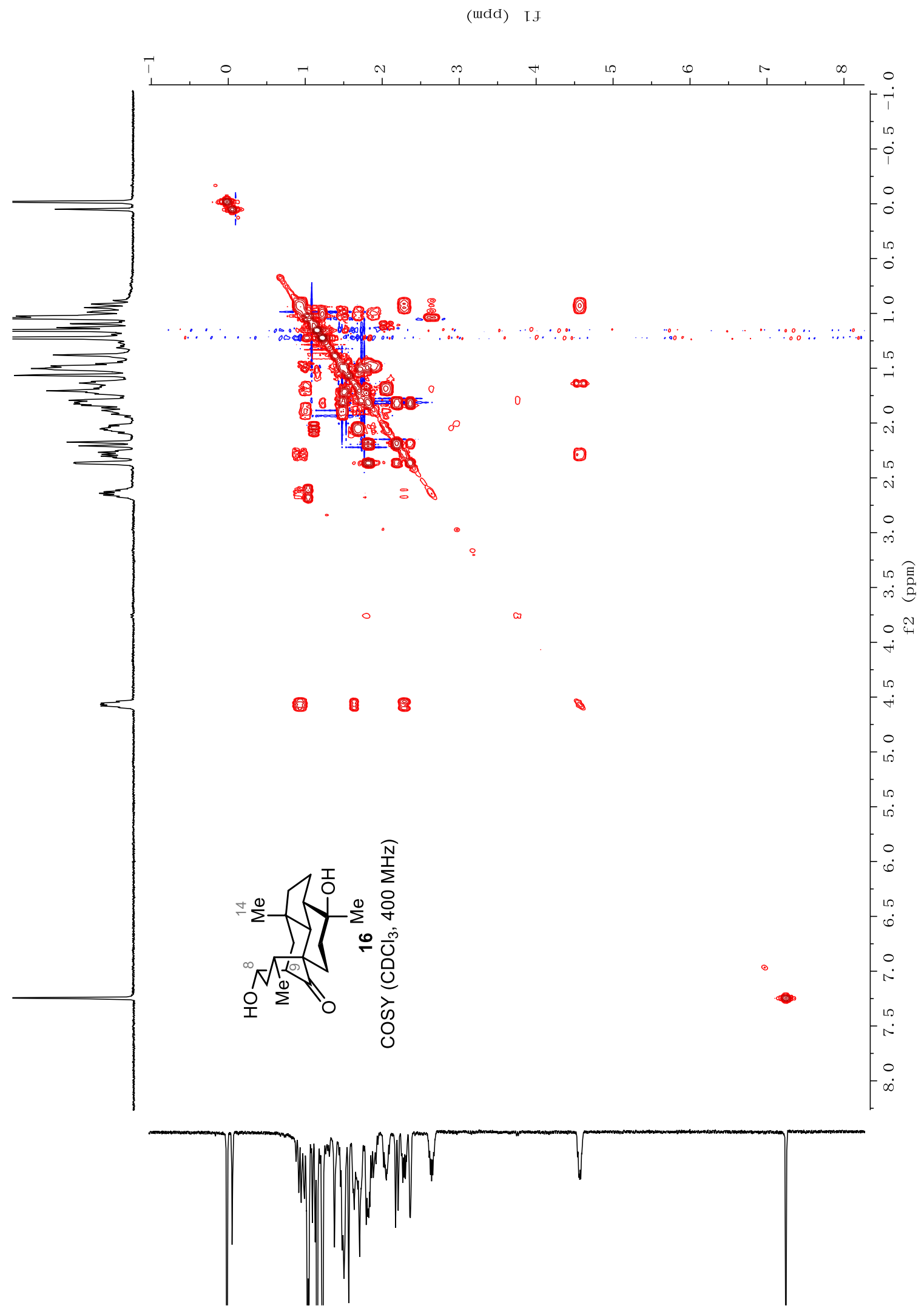




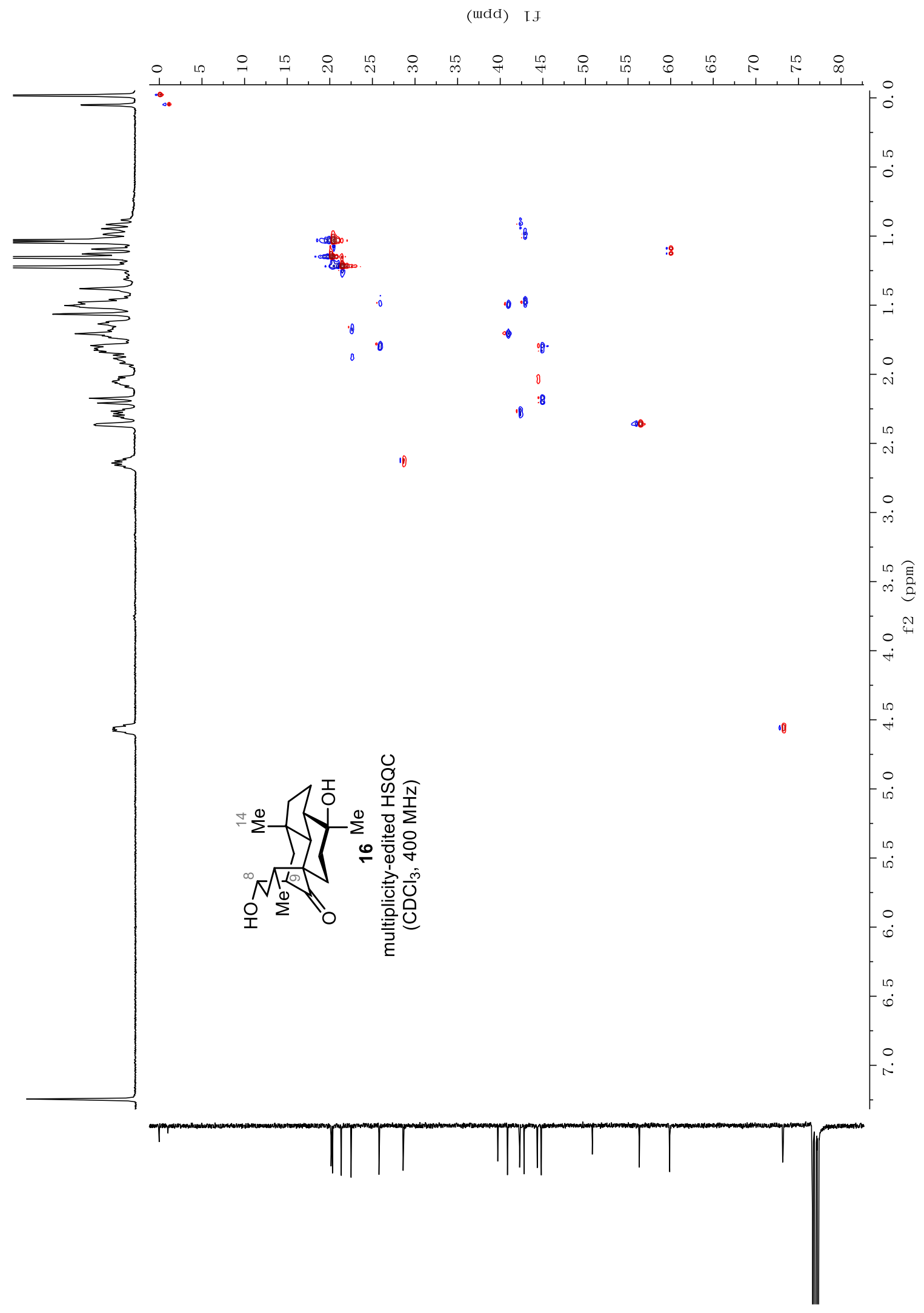




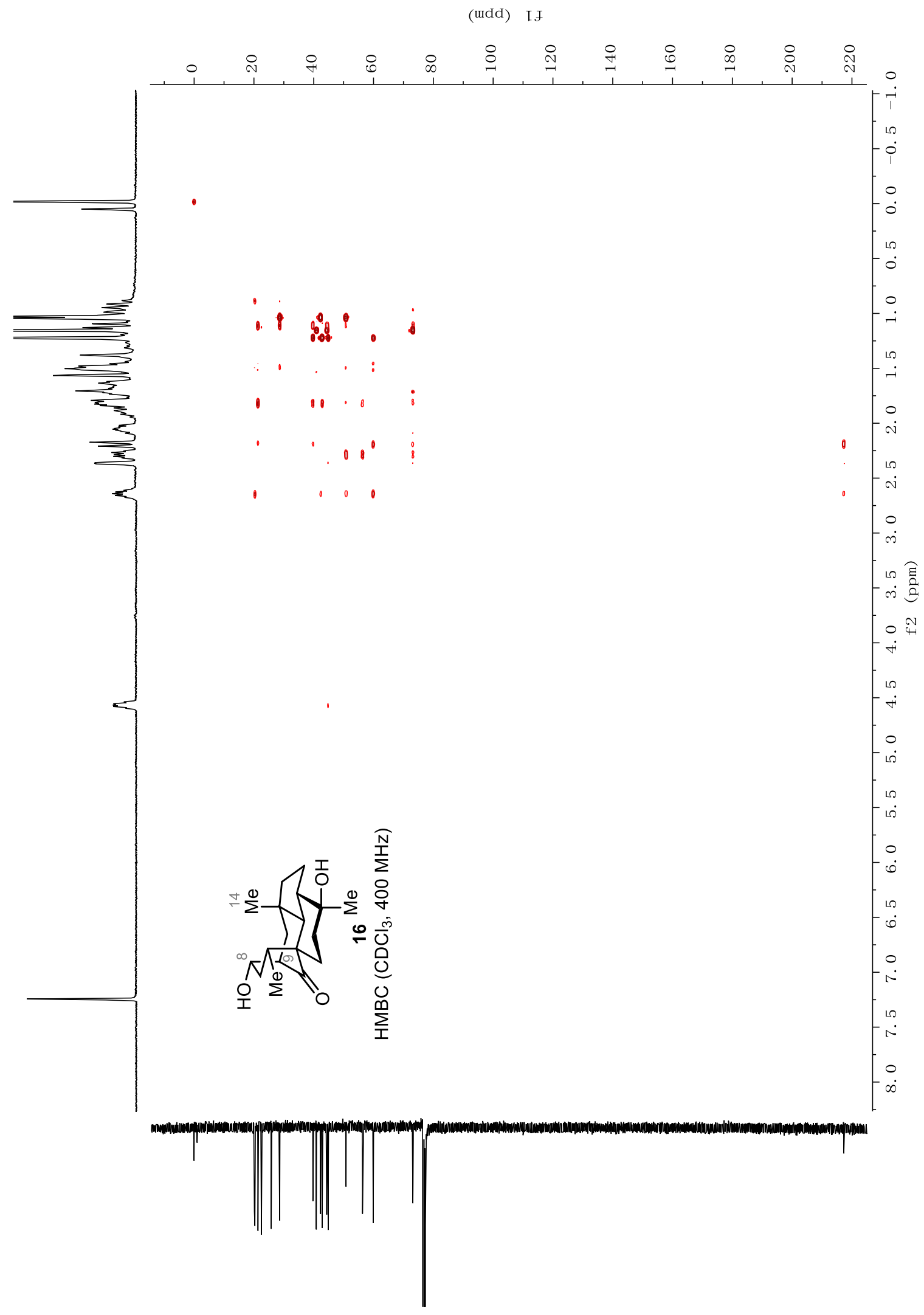




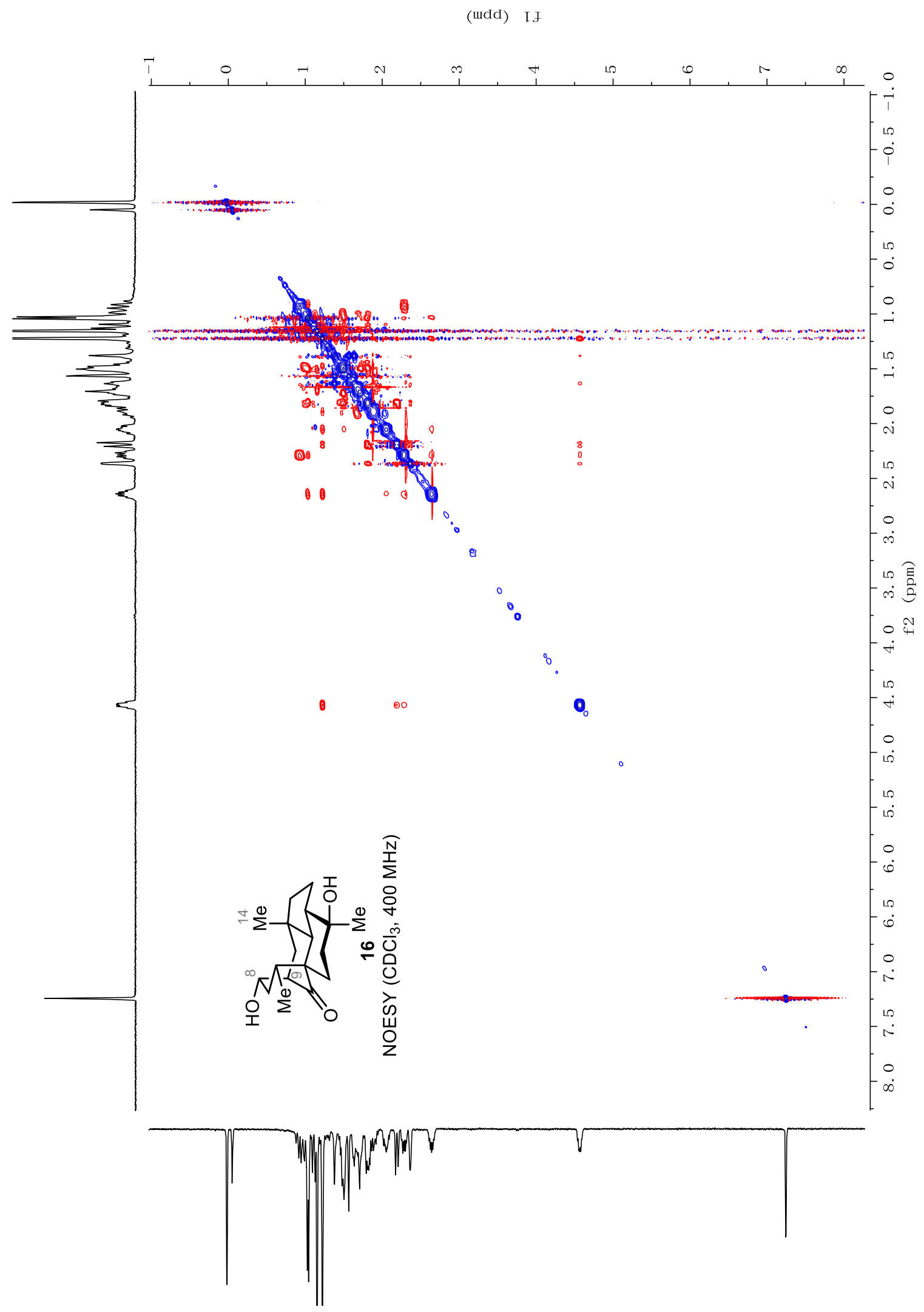



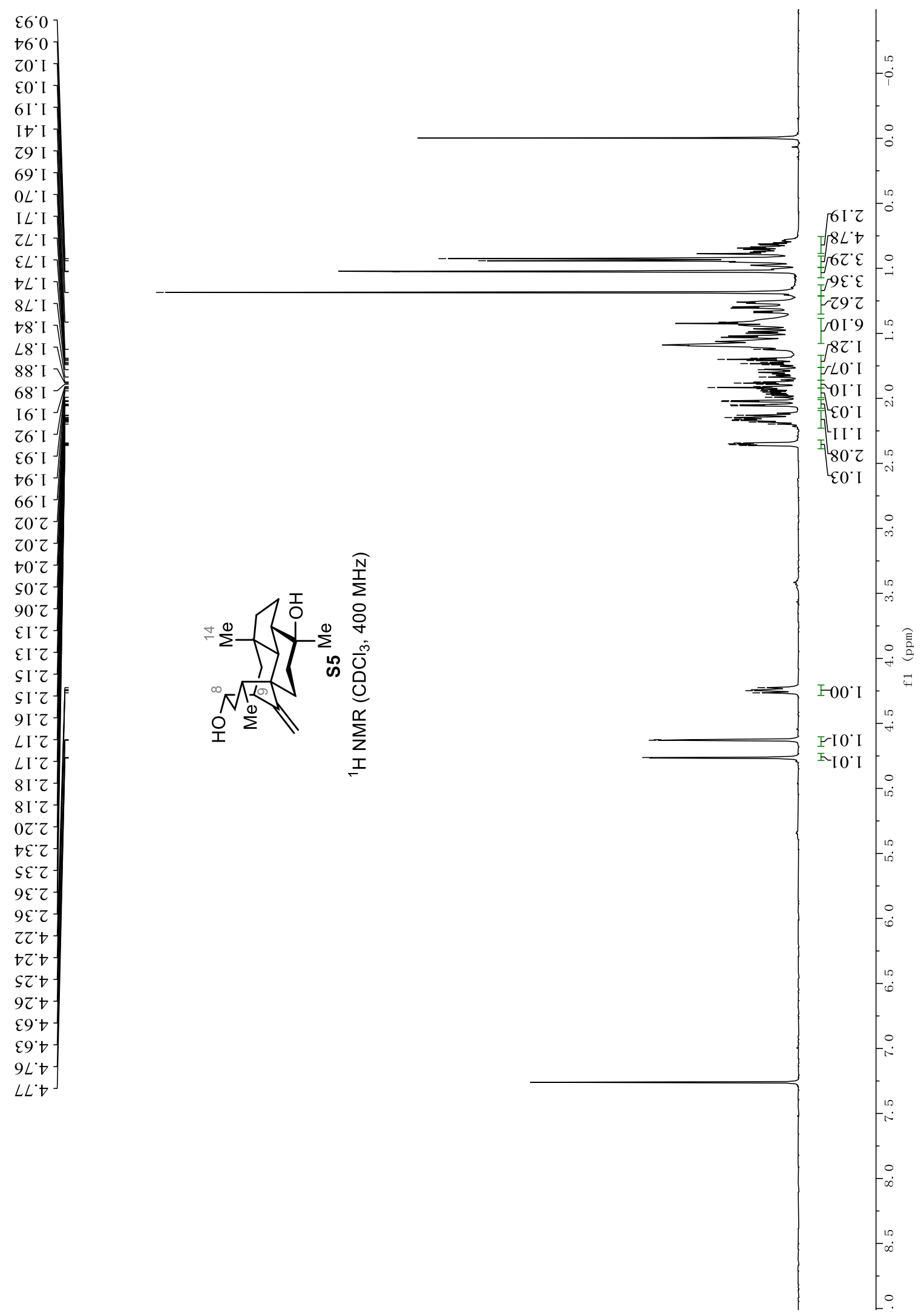


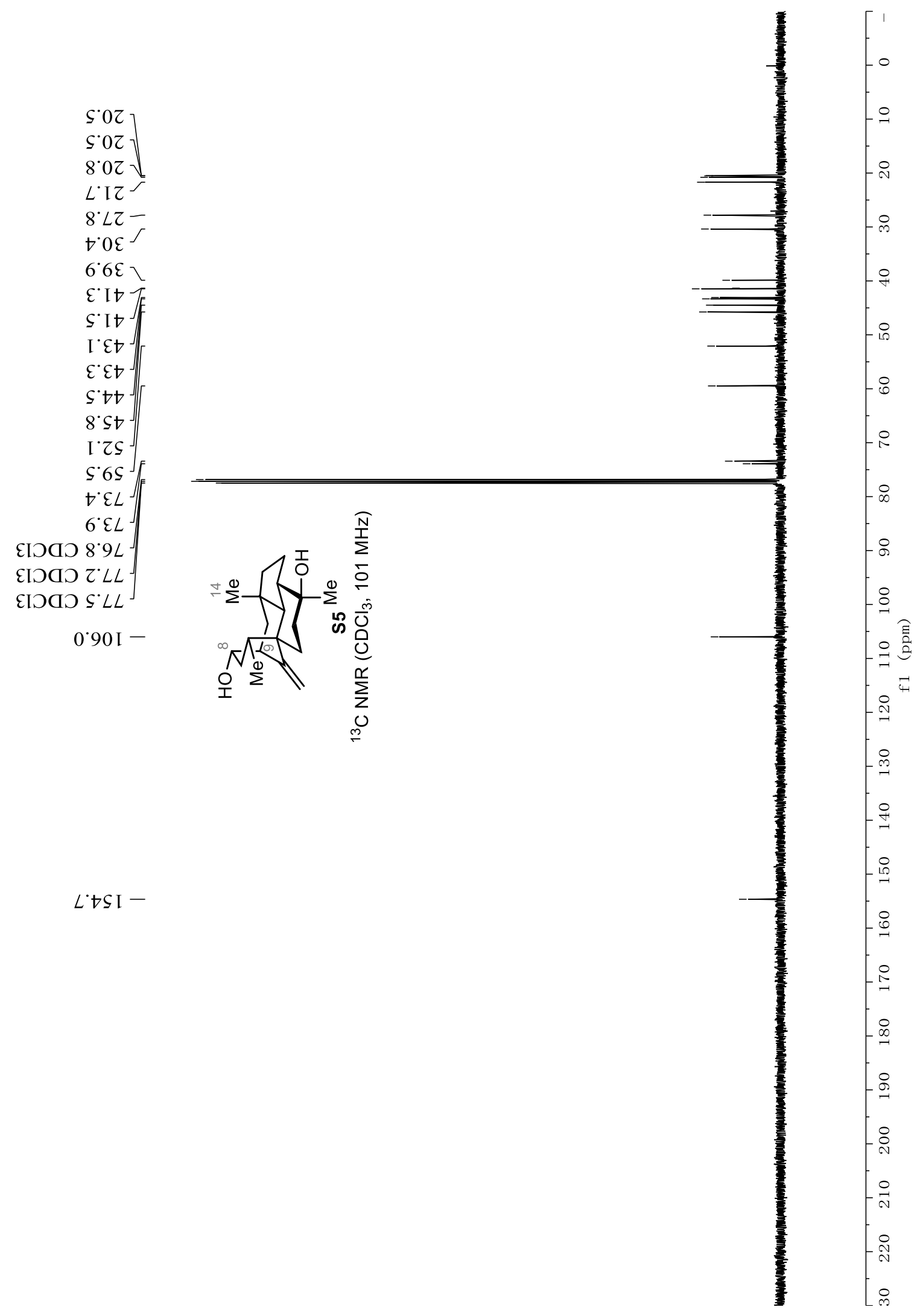




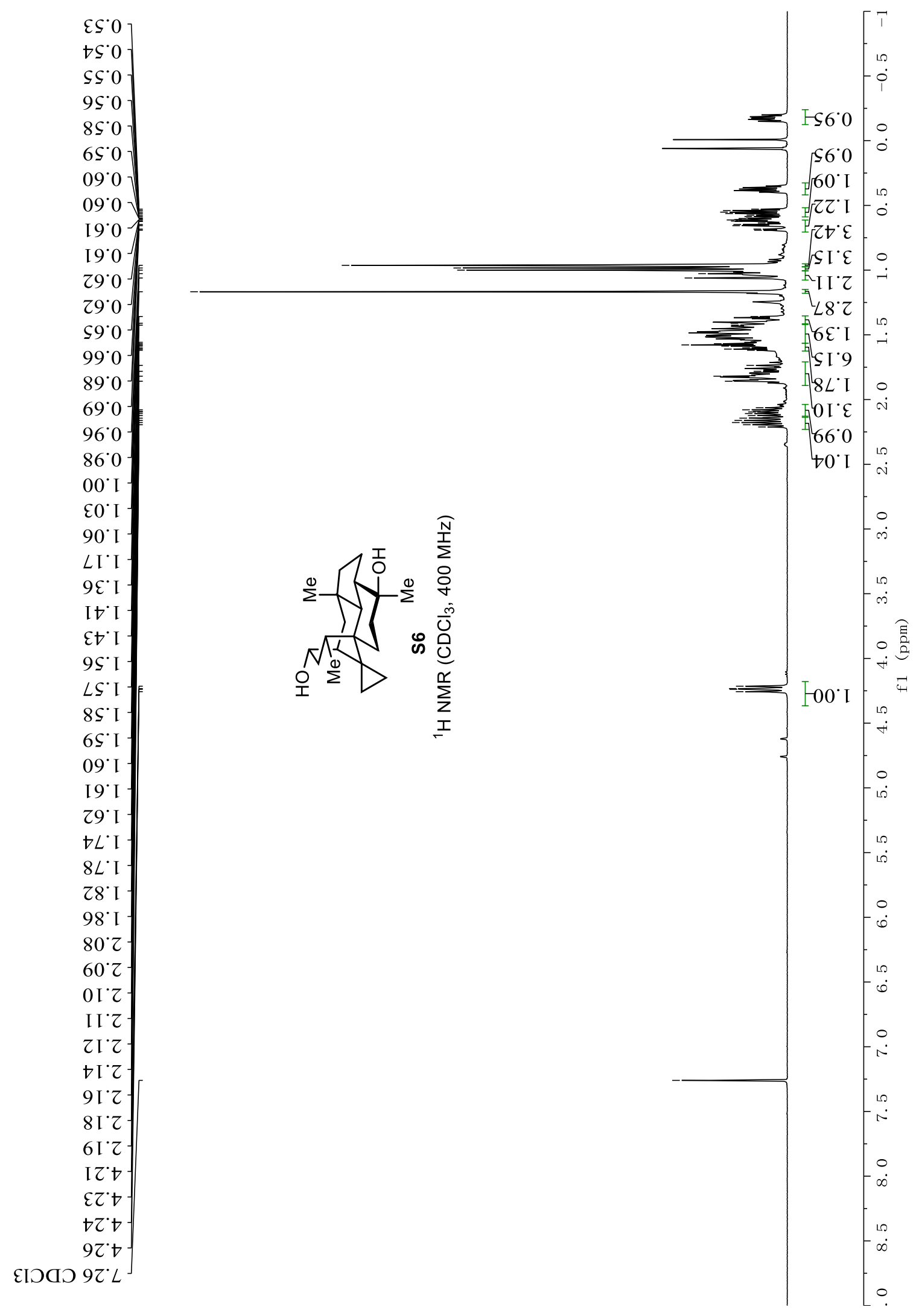



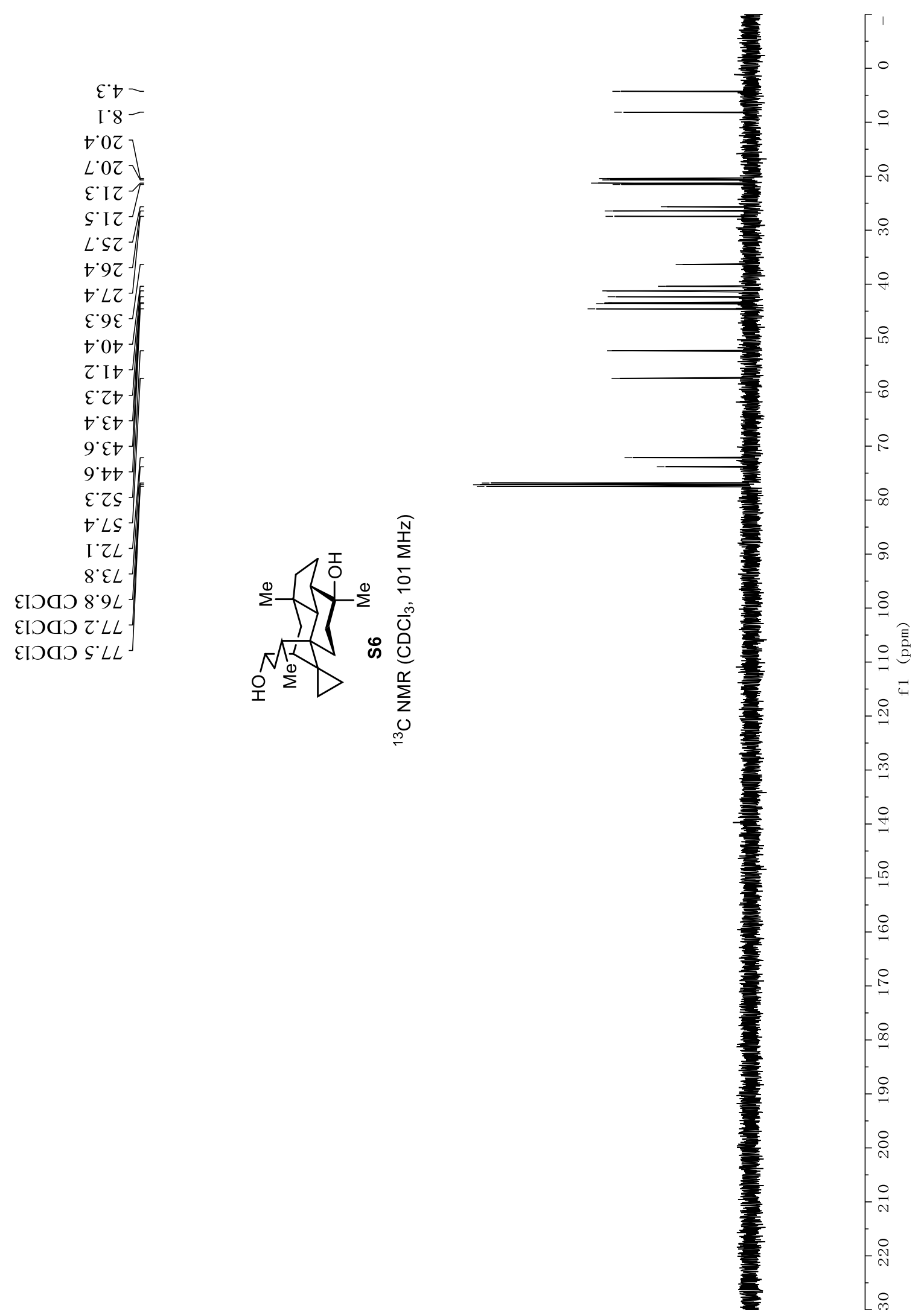


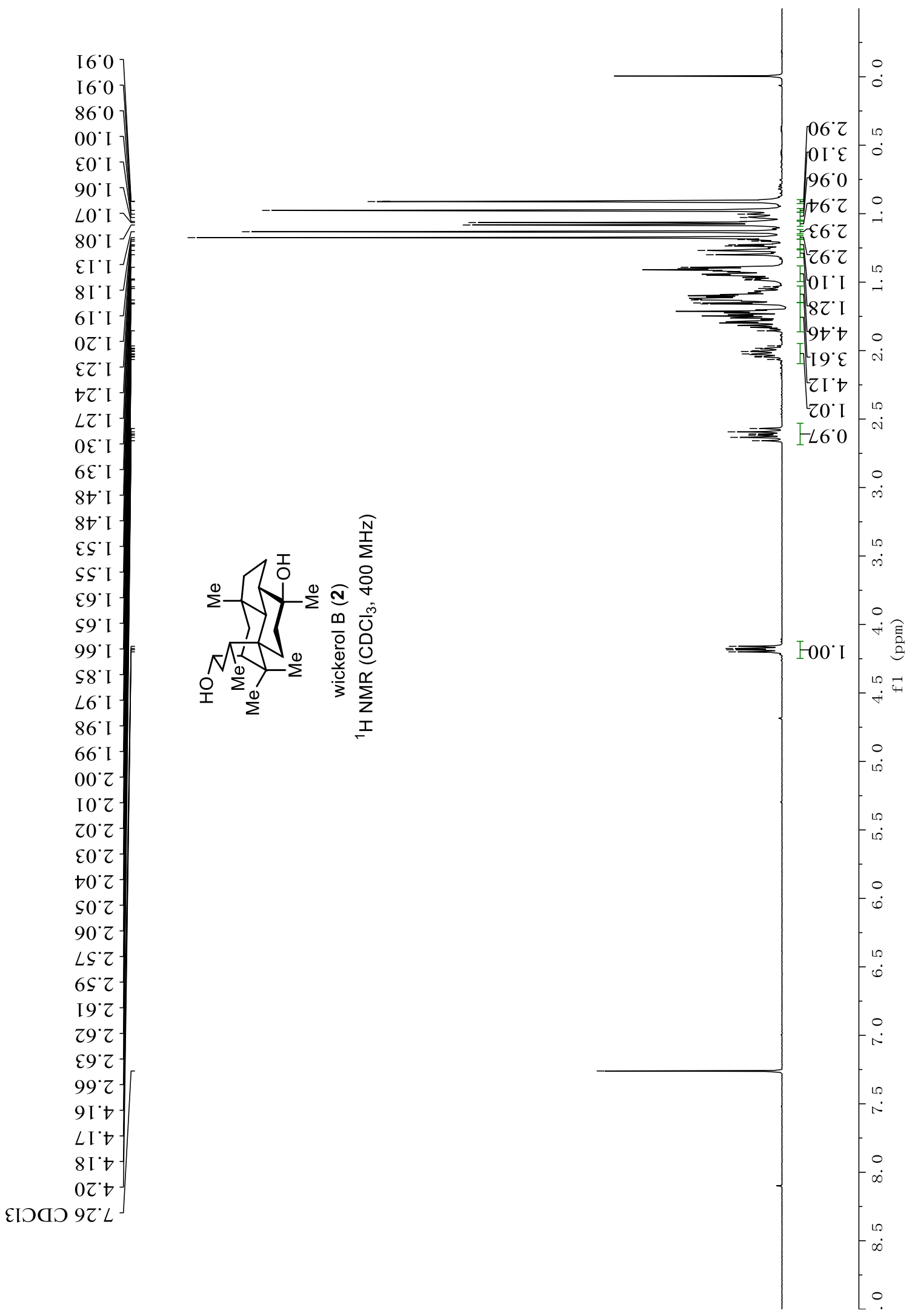



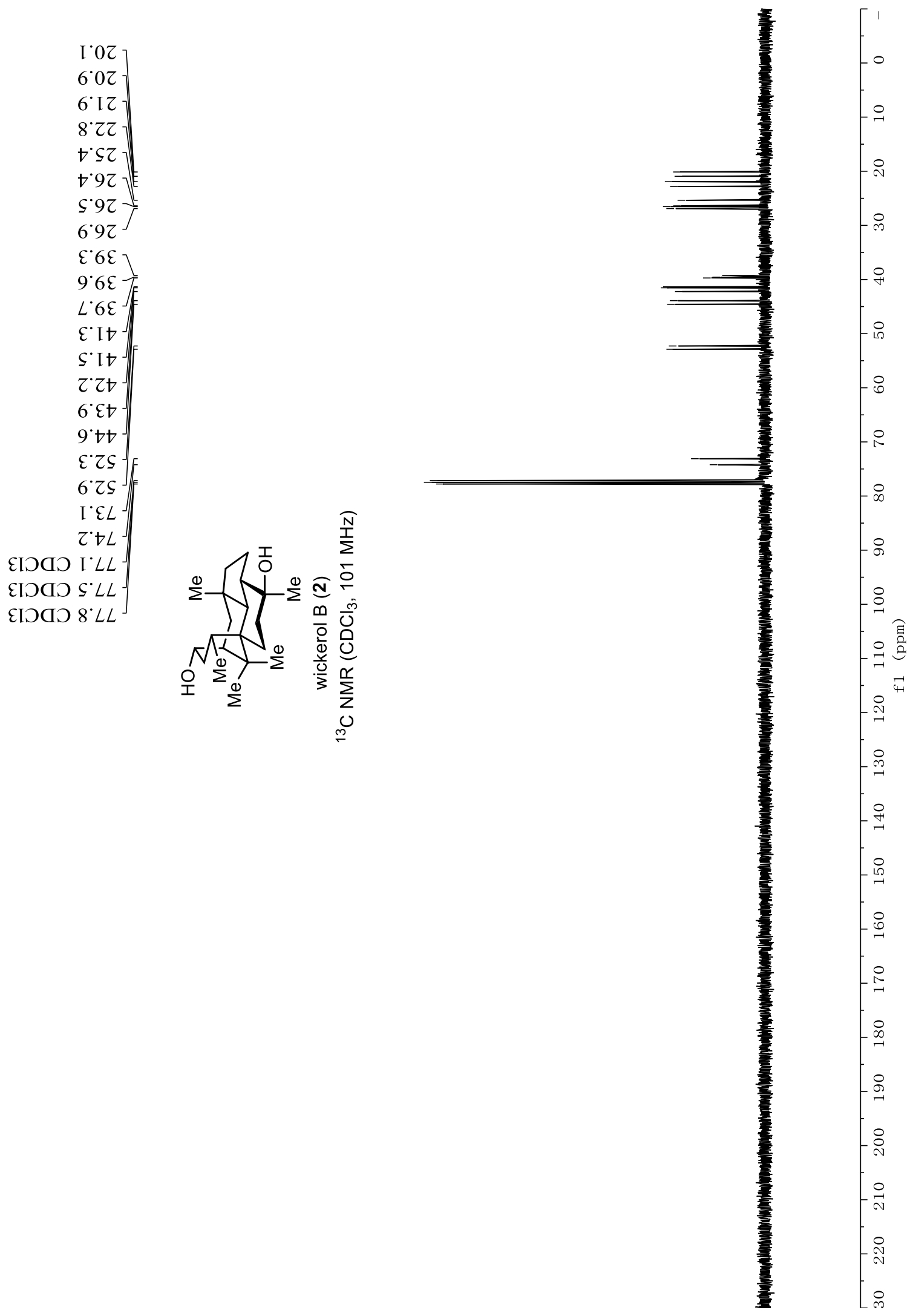


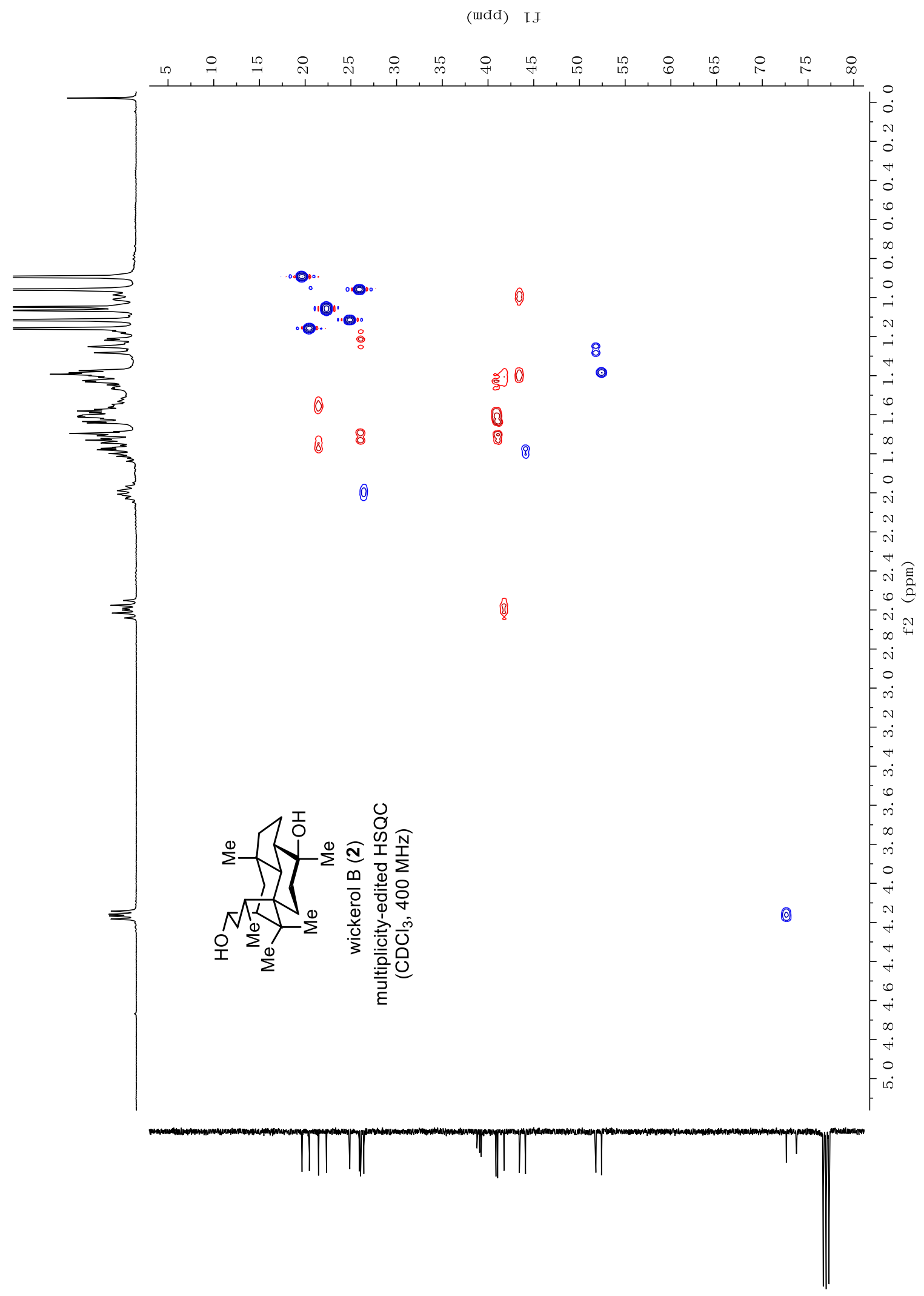




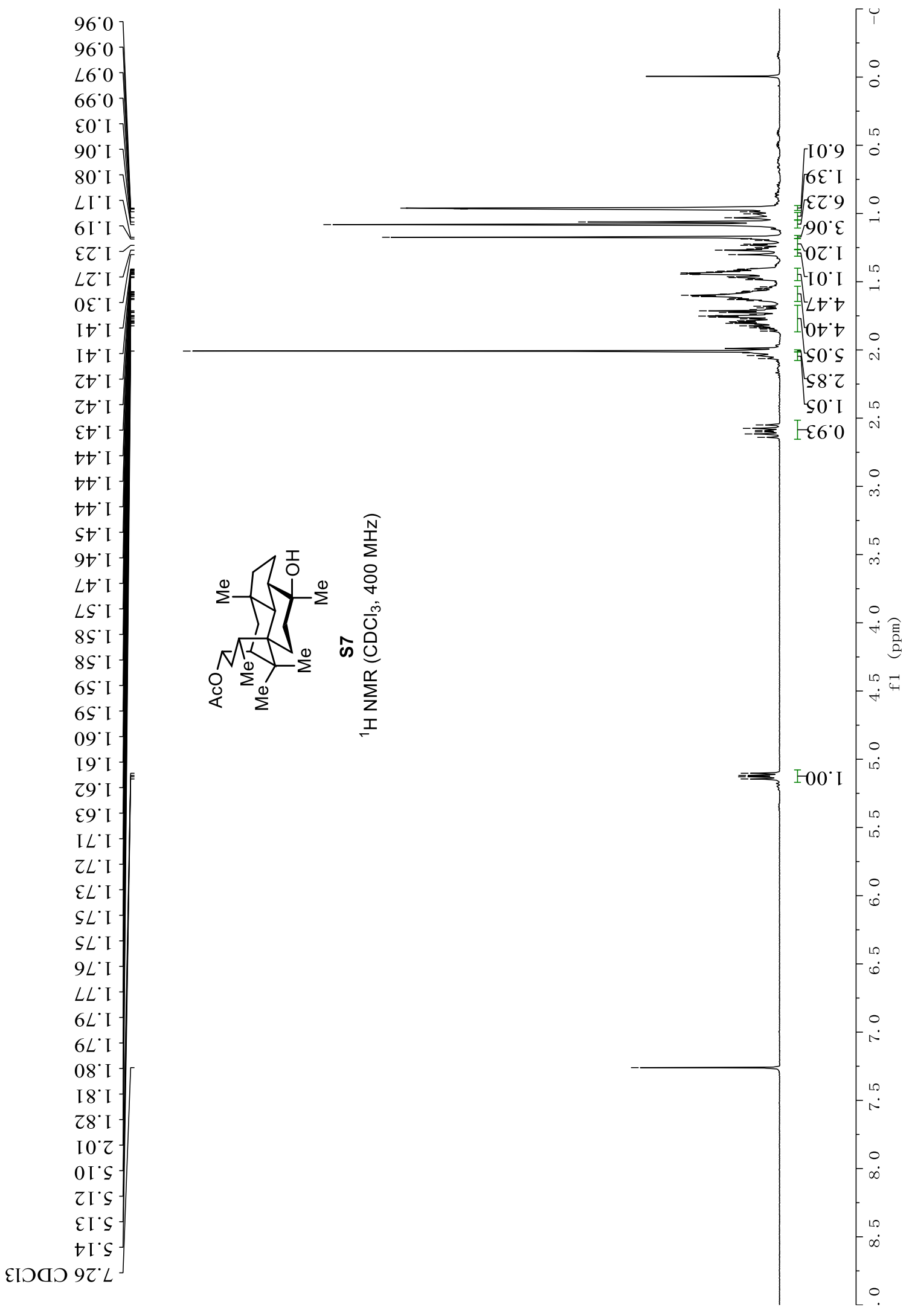




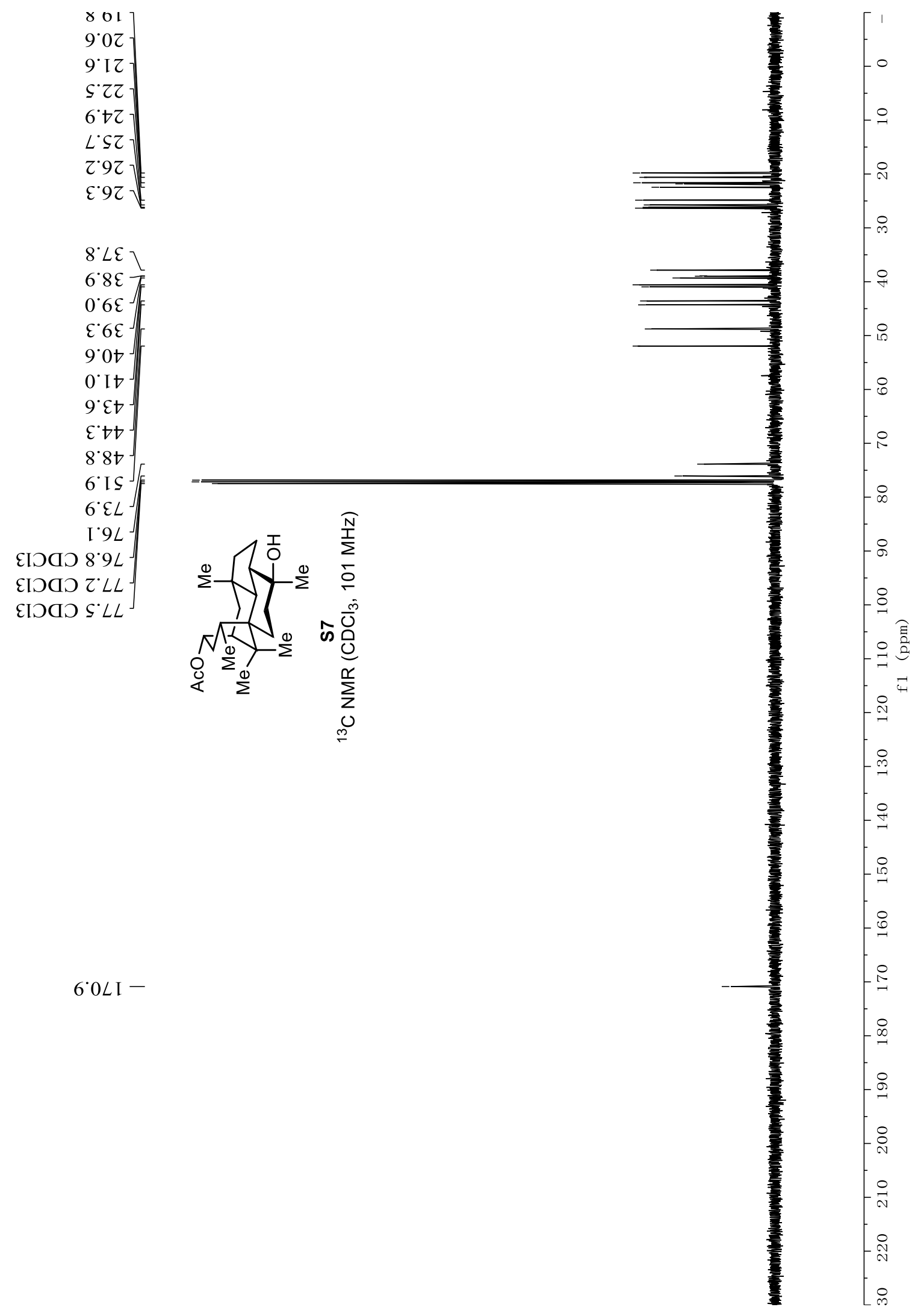




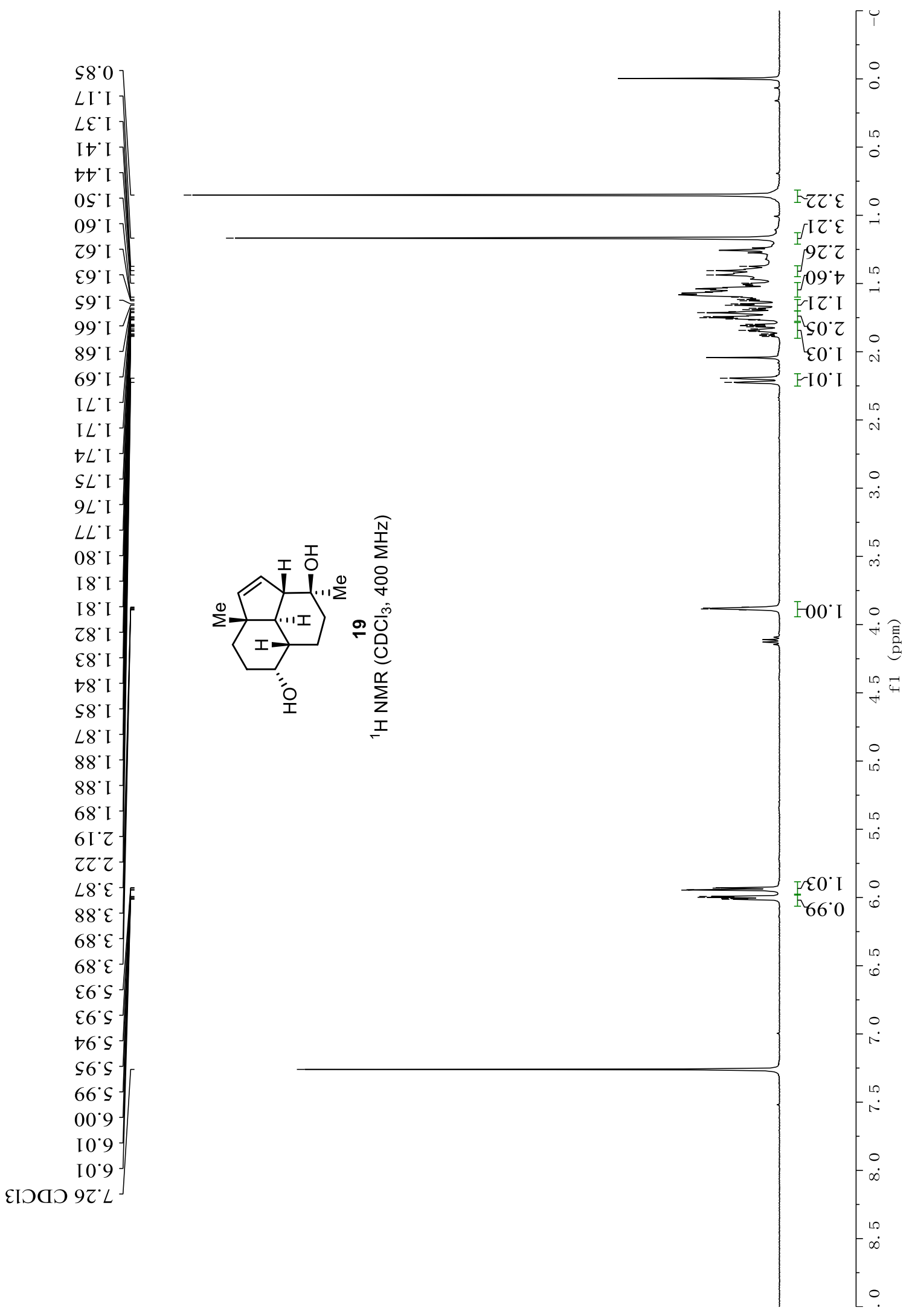




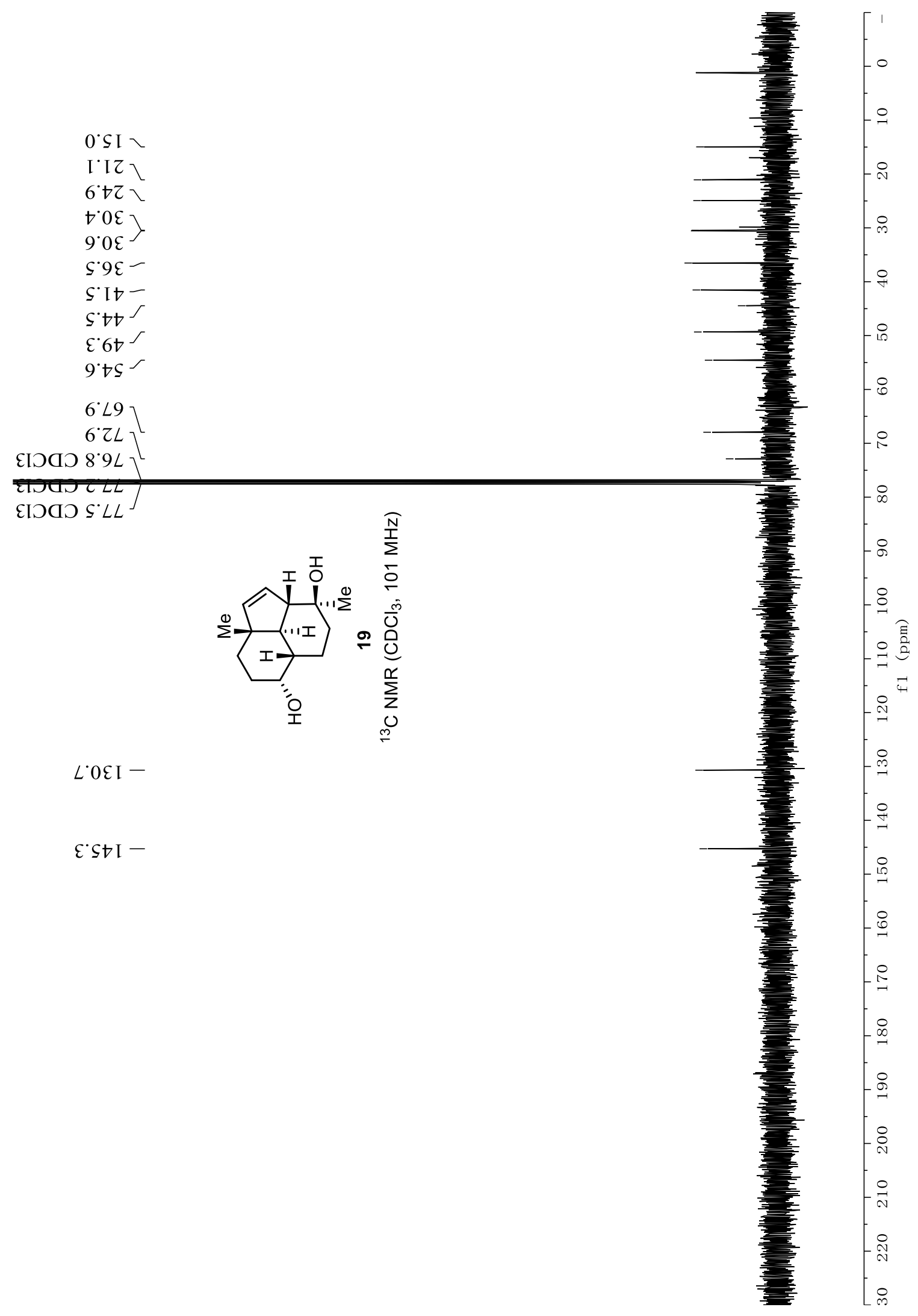




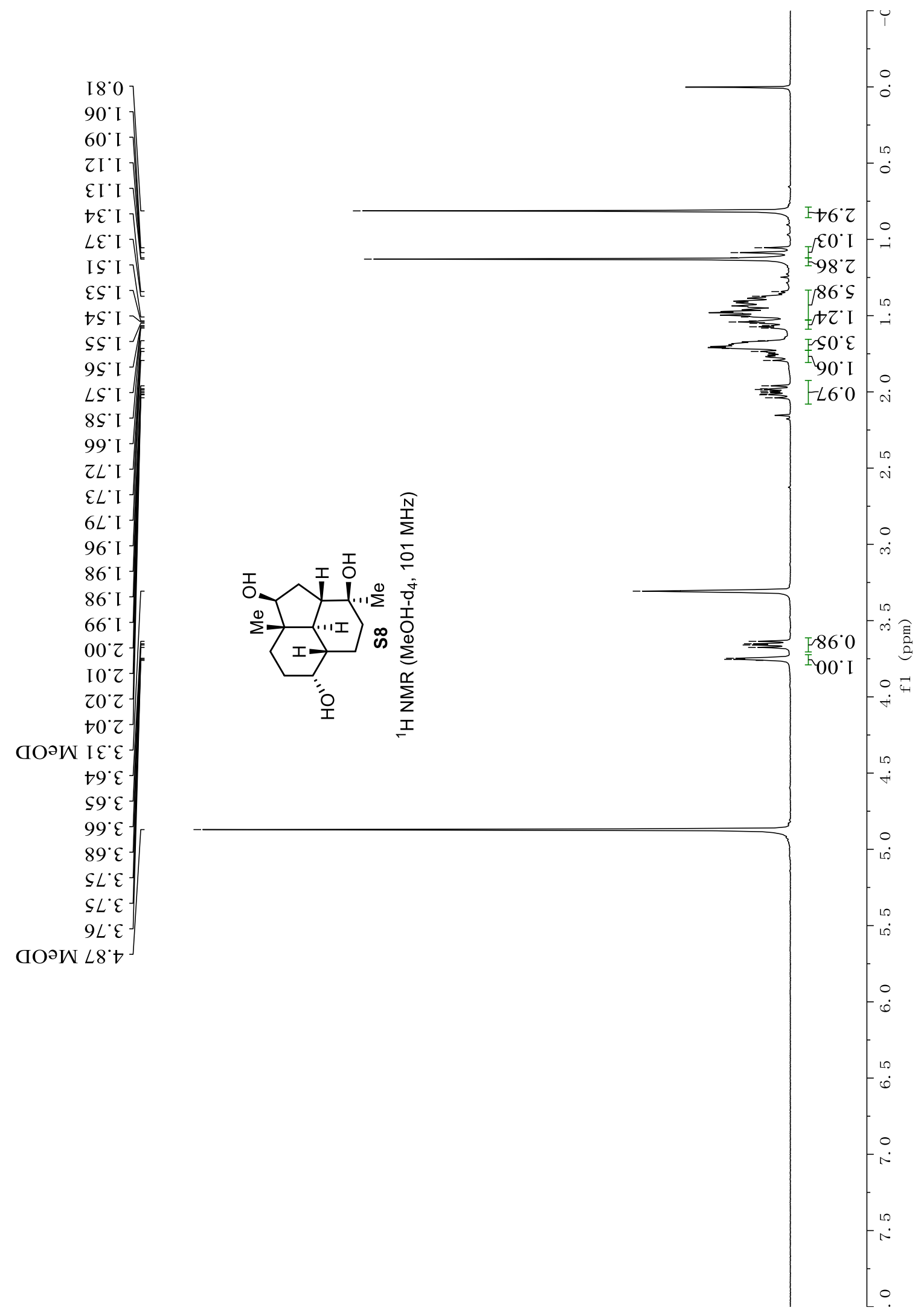




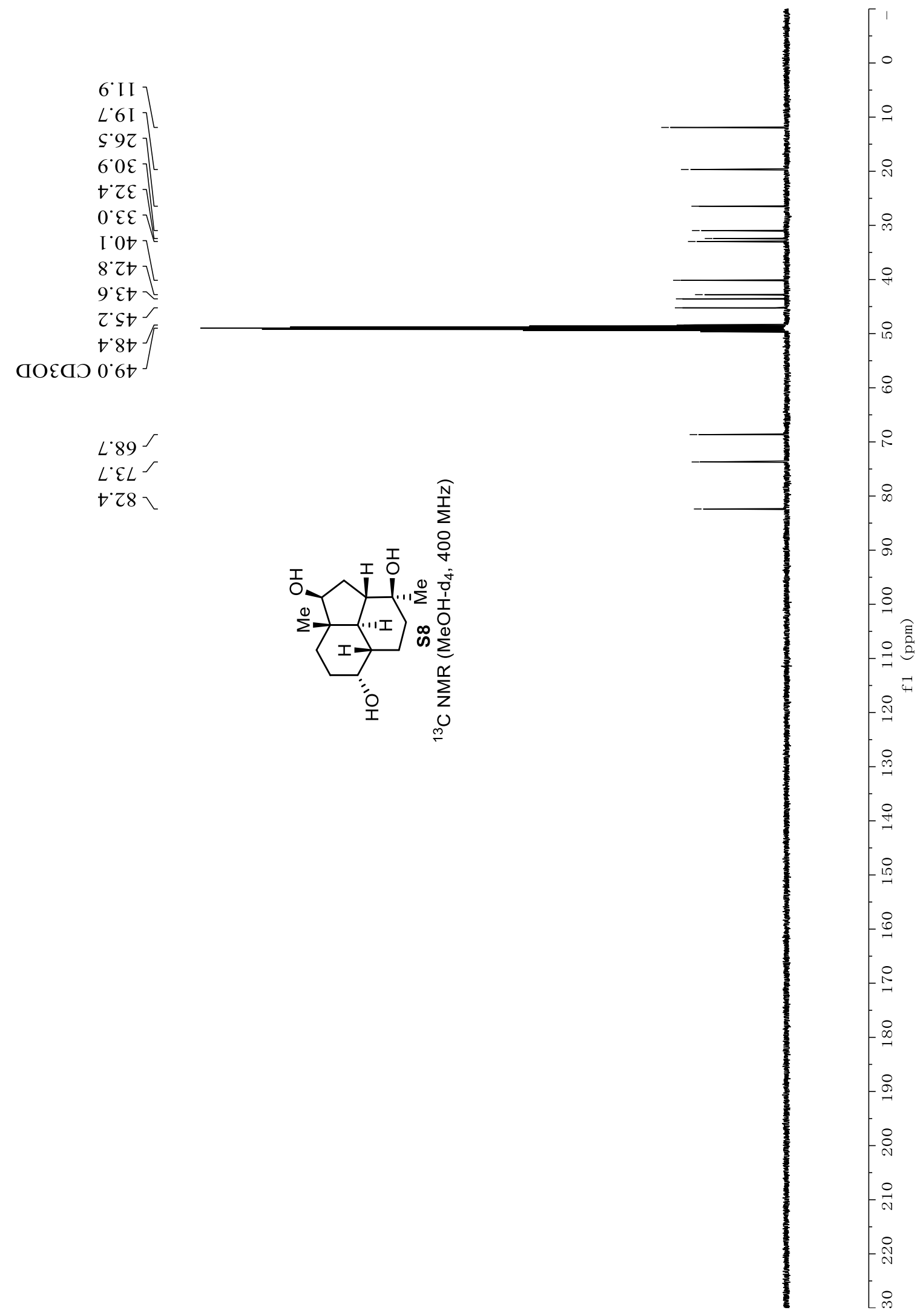




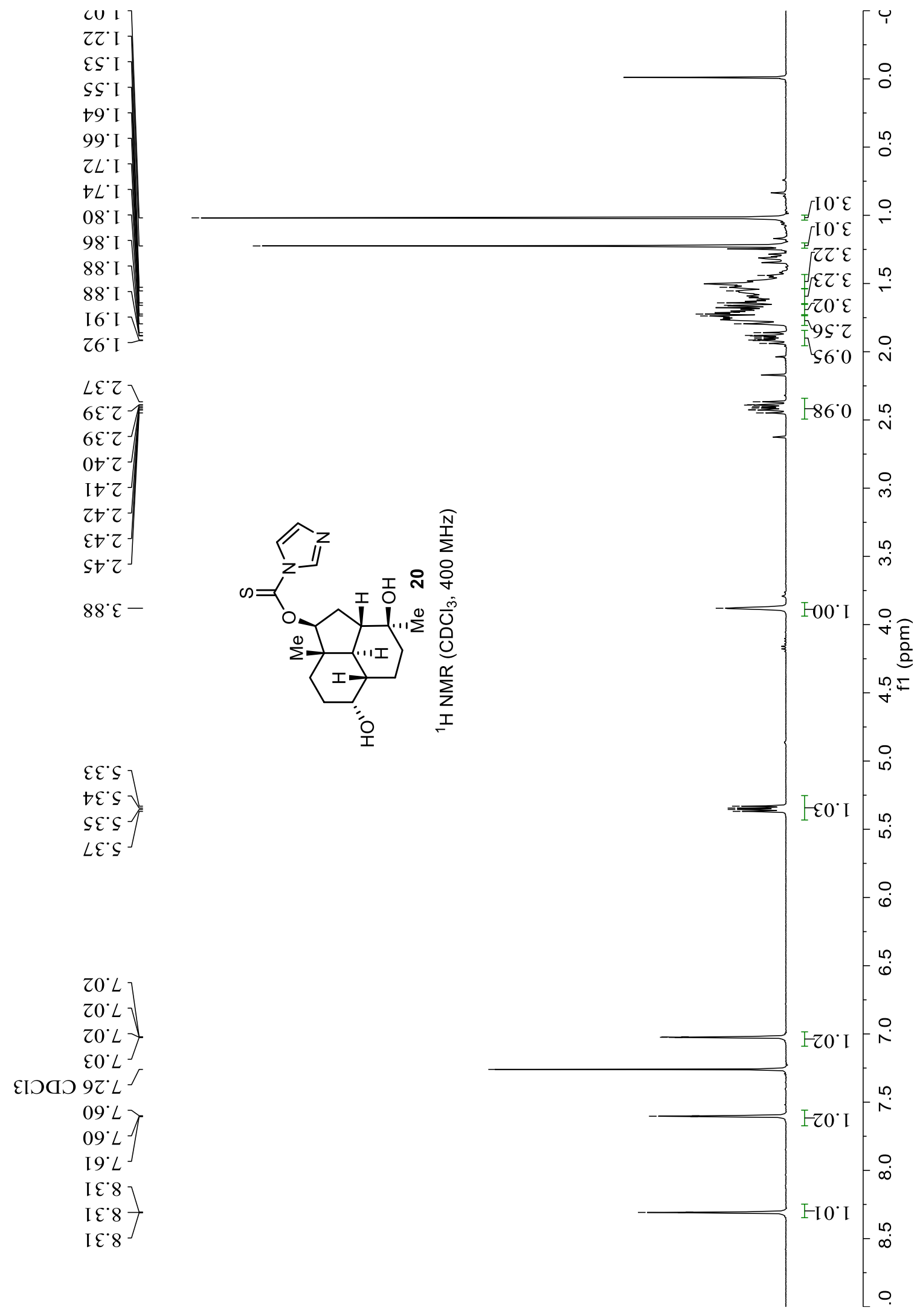




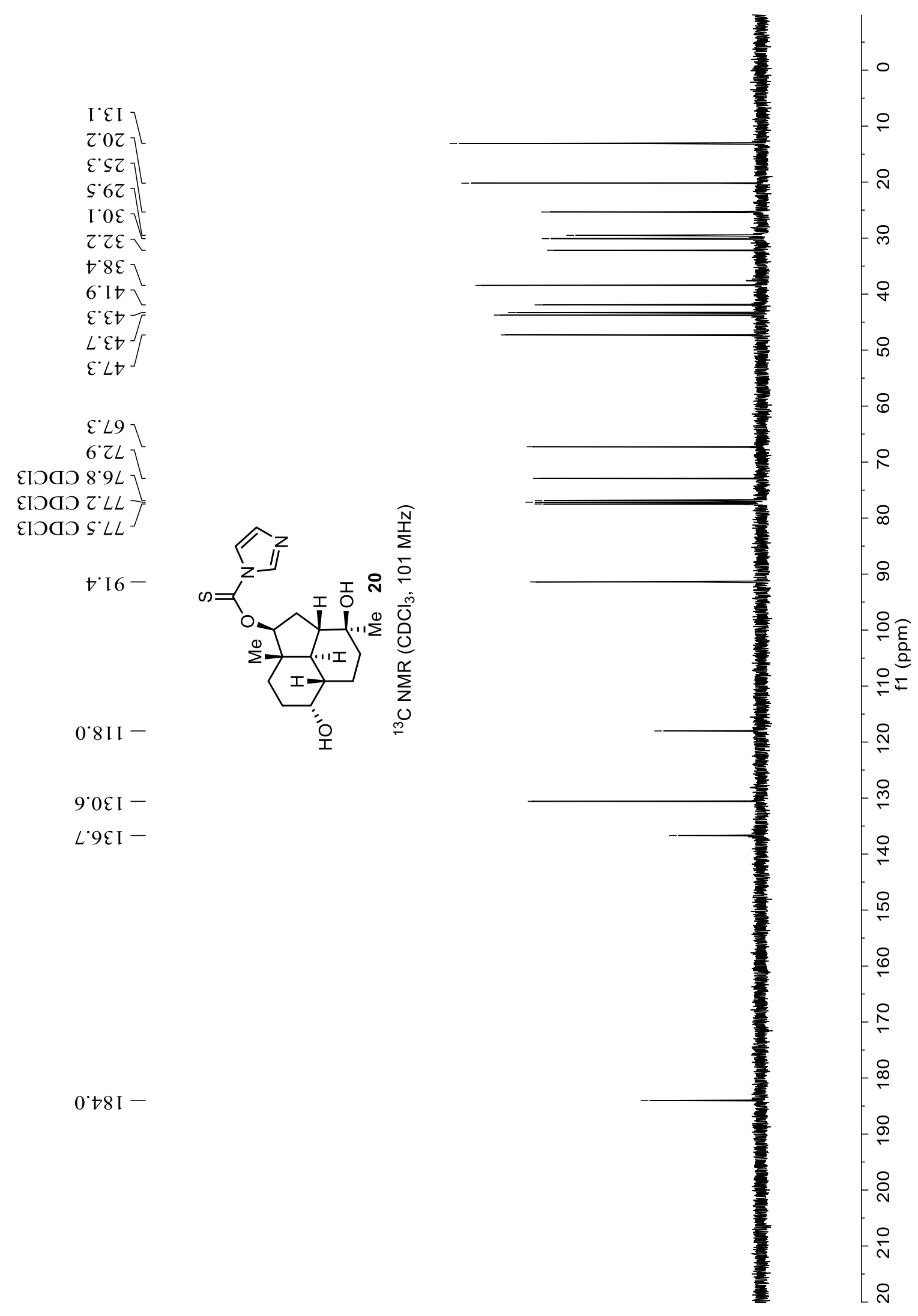




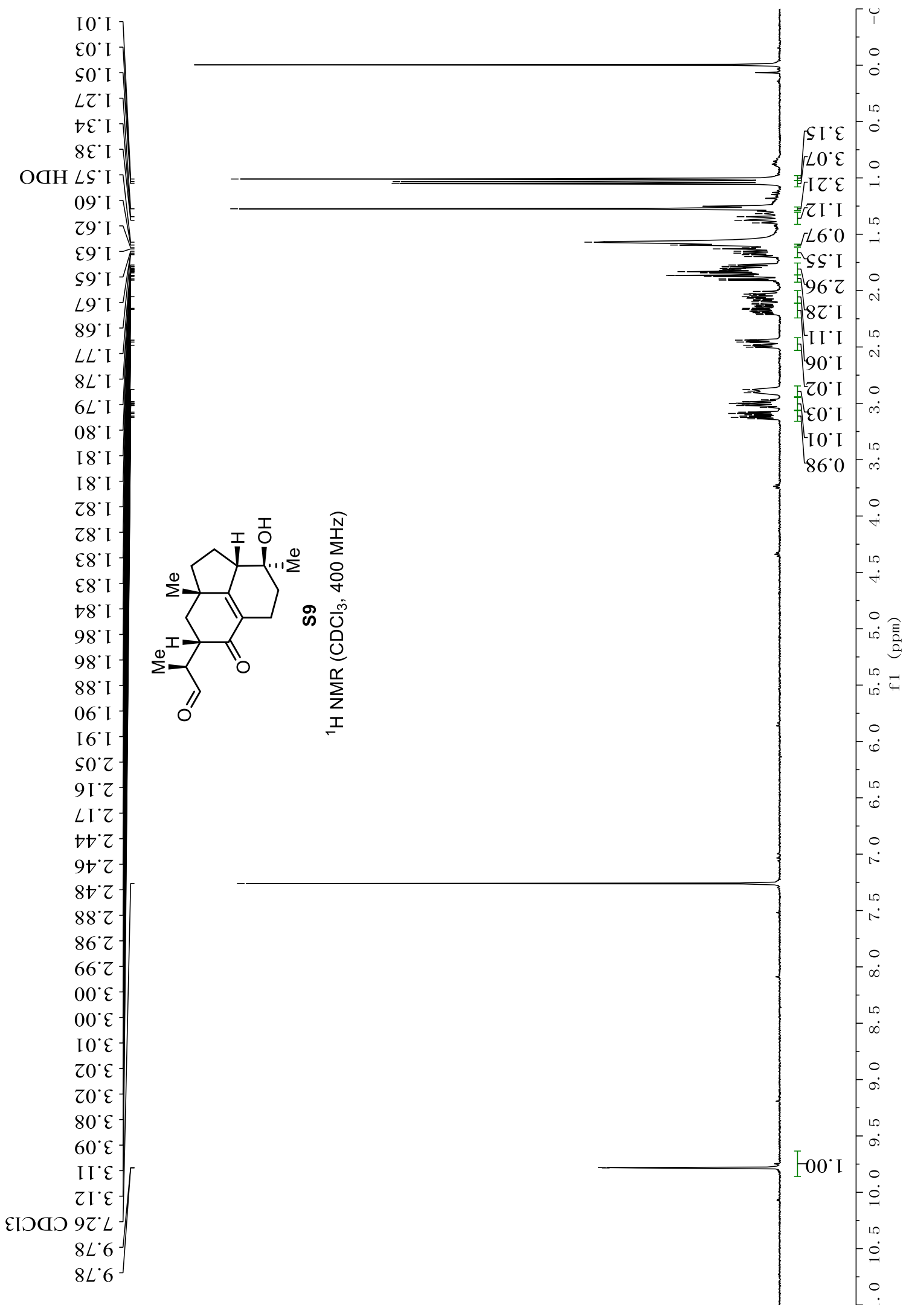




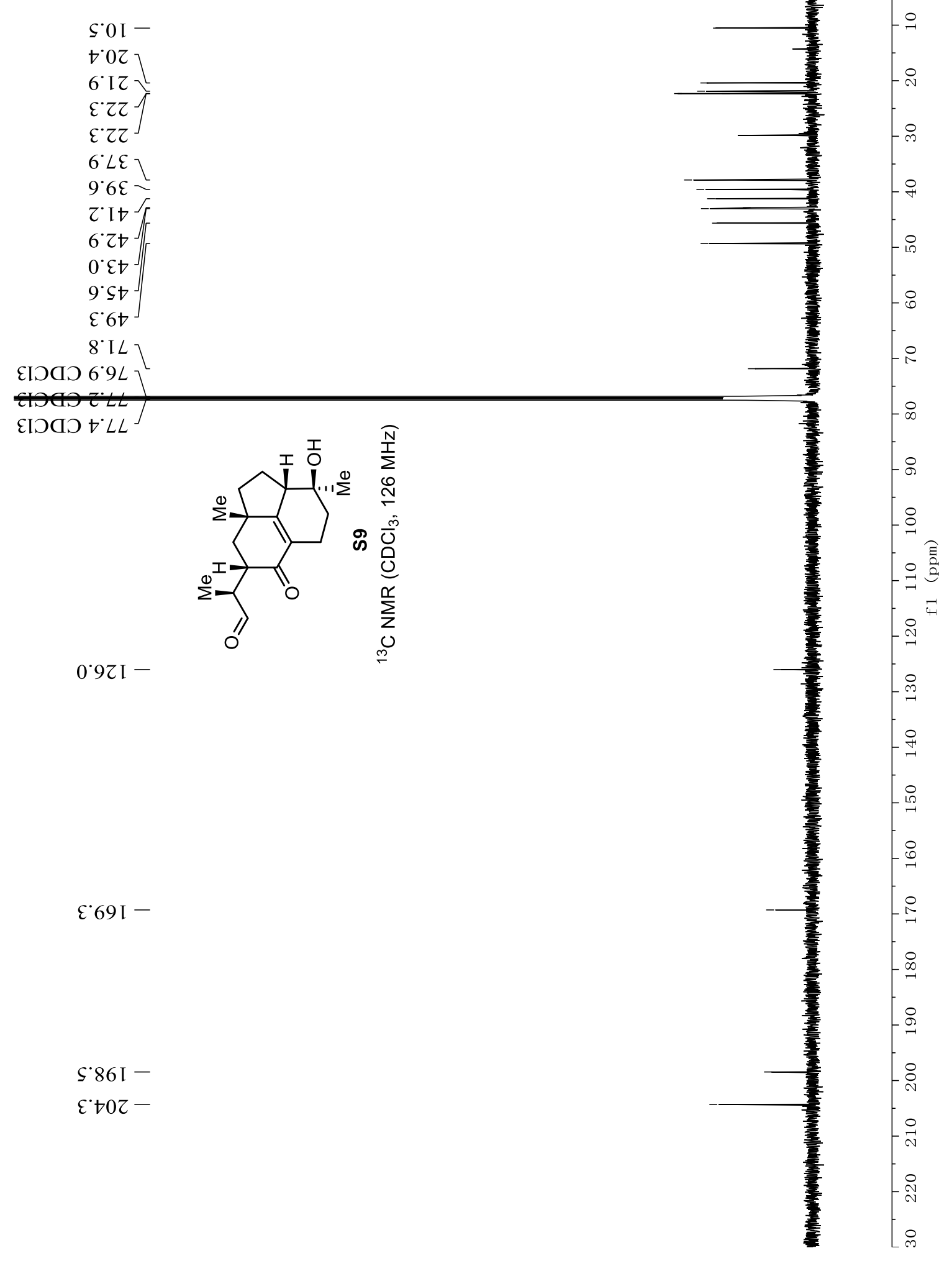




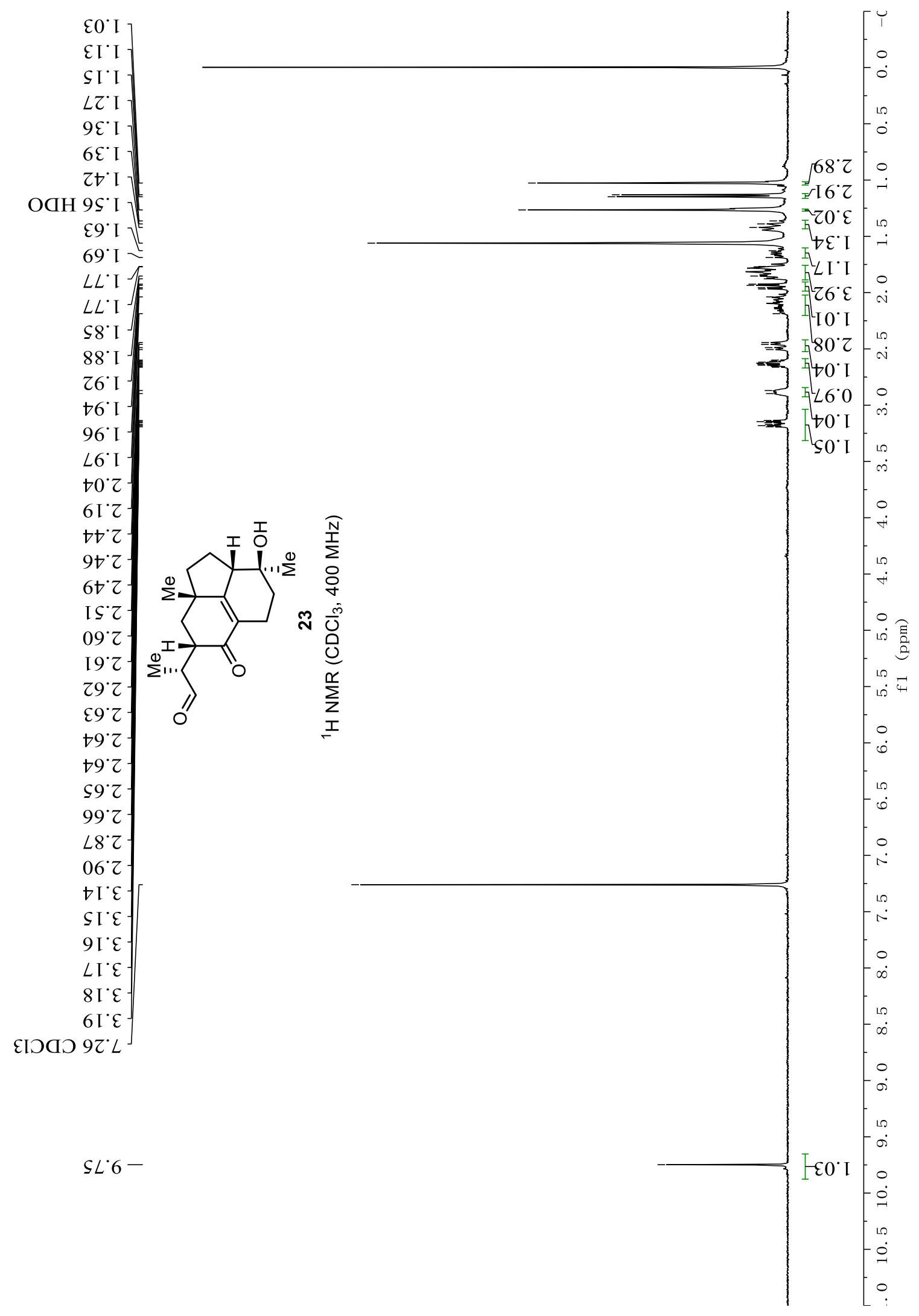




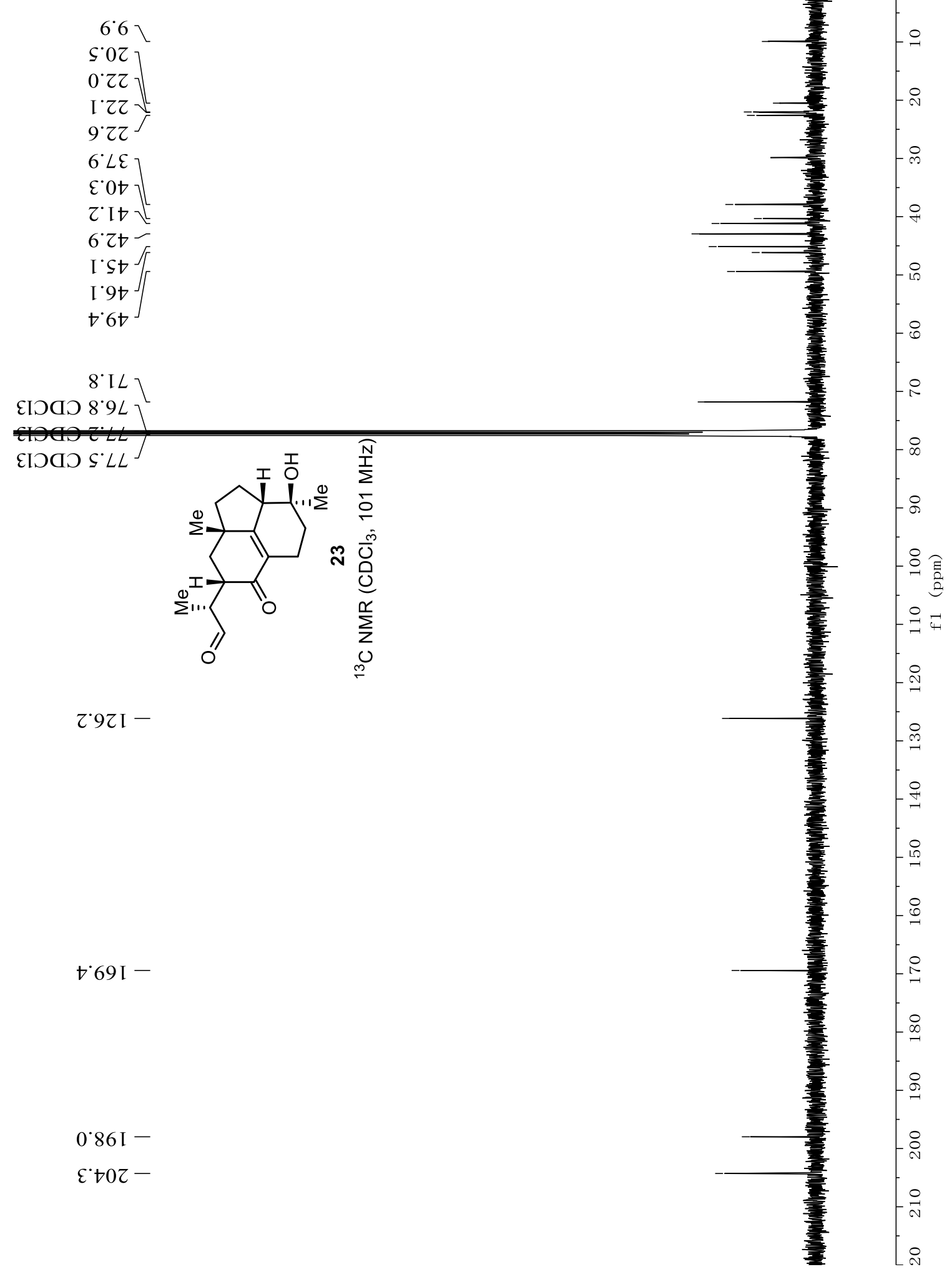




\section{References}

(1) Farney, E. P.; Feng, S. S.; Schäfers, F.; Reisman, S. E. Total Synthesis of (+)-Pleuromutilin. J. Am. Chem. Soc. 2018, 140, 1267-1270.

(2) Szostak, M.; Spain, M.; Procter, D. J. Preparation of Samarium(II) Iodide: Quantitative Evaluation of the Effect of Water, Oxygen, and Peroxide Content, Preparative Methods, and the Activation of Samarium Metal. J. Org. Chem. 2012, 77, 3049-3059.

(3) Frigerio, M.; Santagostino, M.; Sputore, S. A User-Friendly Entry to 2-Iodoxybenzoic Acid (IBX). $J$. Org. Chem. 1999, 64, 4537-4538.

(4) Xu, G.; Renaud, P. Intramolecular Cyclopropanation of 1,4-Dienes through HydroborationHomologation: Easy Access to Bicyclo[3.1.0]hexanes. Angew. Chem. Int. Ed. 2016, 55, 3657-3661.

(5) Yamamoto, T.; Izumi, N.; Ui, H.; Sueki, A.; Masuma, R.; Nonaka, K.; Hirose, T.; Sunazuka, T.; Nagai, T.; Yamada, H.; Omura, S.; Shiomi, K., Wickerols A and B: novel anti-influenza virus diterpenes produced by Trichoderma atroviride FKI-3849. Tetrahedron 2012, 68, 9267-9271.

(6) Sun, P.-X.; Zheng, C.-J.; Li, W.-C.; Jin, G.-L.; Huang, F.; Qin, L.-P. Trichodermanin A, a novel diterpenoid from endophytic fungus culture. J. Nat. Med. 2011, 65, 381-384.

(7) Shi, Z. Chemical Structures and Biological Activities of Secondary Metabolites from Five MarineAlga-Epiphytic Fungi. Ph.D. Thesis, University of Chinese Academy of Sciences, 2018. 